Euskal ikerketen aldizkaria | Revue d'études basques |

Revista de estudios vascos | Basque studies review

Numéro spécial 4 | 2019

SACAZE bildumako euskal testuak (1887)

\title{
Sacaze hiztegia
}

Gotzon Aurrekoetxea, Charles Videgain et Aitor Iglesias Chaves

\section{OpenEdition \\ Journals}

Édition électronique

URL : https://journals.openedition.org/lapurdum/2900

DOI : 10.4000/lapurdum.2900

ISSN : 1965-0655

Éditeur

IKER

Édition imprimée

Date de publication : 1 octobre 2019

Pagination : 207-313

ISBN : 9782955341339

ISSN : $1273-3830$

\section{Référence électronique}

Gotzon Aurrekoetxea, Charles Videgain eta Aitor Iglesias Chaves, «Sacaze hiztegia», Lapurdum [Linean], Numéro spécial 4 | 2019, Sarean emana----an 12 janvier 2020, kontsultatu 22 septembre 2022. URL: http://journals.openedition.org/lapurdum/2900 ; DOI: https://doi.org/10.4000/lapurdum 2900

\section{(c) $\Theta \Theta \Theta$}

Creative Commons - Attribution-NonCommercial-NoDerivatives 4.0 International - CC BY-NC-ND 4.0 https://creativecommons.org/licenses/by-nc-nd/4.0/ 


\section{SACAZE HIZTEGIA}

"Enquête de linguistique et de toponymie des Pyrénées" bildumako euskal testuen hiztegia lortzeko ondoko urratsak eman behar izan dira: a) testu bakoitzeko hitzen zerrenda atera; b) zerrenda bateratua lortu; c) zerrenda horretan azaltzen diren aldaeren artean lematizazioa burutu.

\subsection{Testuetako hitz zerrendak}

Testuak makinatu eta zuzendu ostean testu bakoitzean agertzen diren hitzen zerrenda egin da lehenik. Jardunbide honetan lehen urratsa testuak soiltzea izan da: testuen inguruko xehetasunak (goiburuak, oharrak eta biltzaileei dagozkien ezaugarriak) ezabatu egin dira, testuak bere osotasunean, baina haiek bakarrik landuz.

\subsection{Zerrendetako hitzak datutegiratu}

Herrikako dokumentuetan lortu diren zerrendetako testu-hitz horiek datutegi batean ezarri dira. Testu-hitz bakoitza testuaren zenbakiarekin eta hitzaren agerrera kopuruarekin osatu da. Eraiki den datutegiak aztertzen diren dokumentu guztietako testu-hitz guztiak biltzen ditu. Guztira 51.564 testu-hitz biltzen dira 142 dokumentuetan. Beraz, beste horrenbeste erregistro dituen datutegia osatu da dokumentuetako testu-hitzekin. Testu-hitz hauetan 10.646 aldaera desberdin agertzen dira.

Testu-hitza jaso den herriko laburdurarekin horniturik agertzen da. Testu-hitza herri bakarrean agertu ordez gehiagotan agertuko balitz herri horien guztien laburdura izango du ondoan. Diogunaren adibide gisa abaats testu-hitza begiratzen badugu "aberats" sarrerari dagokion lekuan ikusiko da ondoren "(bes, hel, gab, hel)" herri-laburdurak dituela; hau da, hitz hori Beskoitze, Heleta (bi aldiz), Garrüze herrietan agertu dela. 


\subsection{Testuaren moldatzea}

Lematizazio-lanak hasi aurretik testu-hitzen moldatzeari ekin zaio. Urrats honen bidez hitzak eta unitate gramatikalak doitu egin dira, idatziaren arauetara egokituz: hitzak banandu, banandurik zeudenak eta loturik idazten direnak lotu, idazkera hutsak zuzendu...

Maizen eman diren kasuak aipatzeko bi direla esan daiteke: a) "bat" mugatzailea; b) aditz nagusi eta laguntzailea; c) ezezkako partikula. Hitzen zerrenda egitean kontuan izan den bigarren gauza testuok doitzea izan da; hau da, hizkuntza idatziari dagozkion hitzen mozketak eta hitzen banaketak egin dira. Testuak bere horretan utzi dira, eskuizkribuetan agertzen diren ñabardura guztiekin, bat bera ere aldatu gabe. Baina testuetako hitzak zerrendatzerakoan hitz bakoitza gaur egun euskaraz idaztean erabiltzen diren arauetara egokitu da. Hau gertatu da nagusiki bi kasutan: a) "bat" mugatzailea eta b) aditz nagusi eta laguntzailea. Hala herribatetan bi hitzetan bereizi da "herri" eta "batetan"; orobat, bereizi da zaintzentitzu, "zaintzen" eta "titzu" bestetik ezarriz. Ezezko partikulua ere maiz banandu behar izan da ezdezaten edo ezdienin eta antzekoetan "ez dezaten", "ez dienin" ezarriz. Dena den, ez da beti hain erraza gertatu: badira kasuak zeinetan arau fonologikoa betez agertzen diren: etziuzten, etzakiten, etzuten, etzitiin, etzouten...

Hauez gain, badira kasu bat baino gehiago herri batzuk kontzeptu edo ekintza bat adierazteko bi hitz bereiz idatzita erabili dutenak eta besteetan batean lotuta agertzen direnak; horietako bat "lokhartu" da (batzuetan lokhartu, zein lokartu, besteetan loak hartu). Hauetan eskuizkribuan agertzen diren bezalatsu utzi dira, nahiz lematizazioan lema bakarraren pean ezarri.

Idazkera hutsak ez dira testu makinatuan zuzendu ezta datutegian ere. Baina lematizaziorako oso kontuan izan dira eta lema ezartzean ustezko idazkera zuzenean lematizatu da.

\subsection{Zerrenda bateratuko aldaeren lematizazioa}

Azkenik, lema bakoitzari agerrera-kopurua ere ezarri zaio. Honen bidez hitz bat testu bakoitzean zenbat aldiz agertzen den jakin dezakegu. Eta aldi berean hiztegian lema edo sarrera bakoitzaren agerrera-kopurua ezartzeko lagungarri egiten da.

Testu-hitzak behin datutegi batean kokatu eta doitu ondoren hitzen lematizazioari ekin zaio. Lan hau proiektu honetarako eraiki den lematizatzailea erabiliz egin da. Ezaguna da euskarari egokitutako lematizatzaile automatizatuak badirela; hala ere, corpus honen ezaugarriak kontuan hartuz (euskara dialektalean eta idazkera ez-estandarrean idatzia...) aplikazio informatiko berezi bat egitea erabaki da. Izan ere, eskuarteko testuak ez dituzte betetzen grafia zein morfologia aldetik idatziaren arauak. Merkatuko programak erabiltezin bihurtzen dituzten eta programa berezia eraikitzera behartu gaituzten arrazoiak ondokoak dira:

a) Grafia desberdinak 
Hitz bera modu grafiko askotan idatzia agertzen da testuotan. "aberats" hitza, adibidez, ondoko aldaera grafikoetan agertzen da: abaats, abaatsac, abaatsak, abarats, abaratsac, abaratxac, abarax, abats, abâtsac, abatsac, abatsak, abatz, abeassak, abéatch, abeatchac, abéatj, abéatjac, abeats, abéats, abeatsak, abeatx, abéatx, abeatz, abéatz, abeax, abeaxac, aberac, abérach, aberas, aberasac, aberassac, abératçac, aberatch, abérats, aberats, aberatsac, abératsac, aberatsak, abératss, abératssak, abératx, aberatx, aberatxac, abératz, aberatz, aberatzac, abératzak, aberatzak, aberax, abérax, aberaxac, aberaxak, aberaz, abérazac, abetx, eberax, habeatchac. Denak "aberats" lemapean ezarri dira.

b) Hitz homomorfoak

Forma bera izan arren kategoria gramatikal edo adiera desberdina duten hitzak azaltzen dira sarri dokumentuetan. Holakoen artean kokatzen da, adibidez behar hitza.

c) Hitzen mozketa desberdinak

Hitz eta unitate gramatikalen mozketak arazo bat baino gehiago ekarri du testuon tratamenduan. Era honetako arruntenak aditz nagusi eta laguntzailearen artean, batetik, eta izen edota izenondo eta zenbatzailearen artean, bestetik, agertu dira. Alderantzizko fenomenoak ere jasotzen dira: gaur egungo usadioan loturik idazten direnak testu batzuetan bereizirik ere ager daitezke. Ez denetan ordea, testuen artean desberdintasun handiak agertzen direlako. Hauen artean aipagarri gertatzen dira adibidez, gorago aipatu den "lokartu" hitzaz gain, "supazter" kontzepturako aldaera askeak chou bazterrean, sou pazterrian bezala, aldaera lotuak chubasterrian, soubazterrian, soupazterrian, souphasterrian, subasterrian bezala, zein marra bidez lotuak su-bazterrean bezala.

d) Hitzen aldaera desberdinak

Aldagarritasun handia agertzen da testuetan zehar. Hain eremu txikia izanik hitzen aldaeretan oso ugaria da bildu den corpusa. Horien adibide gisa gorago aipatu den "aberats" hitza berrartu daiteke.

e) Kontrakzio edo laburdurak

Ahozkoaren ispilu diren dokumentu hauetan kontrakzio asko agertzen da. 'Behar' da laburdurak eragiten dituen unitate adierazgarrienetako bat. Asko eta era askotakoak agertzen ditu: beaüc, beauk, beaun, béhaouc, béhaoun... Maizen gertatzen diren kontrakziook nagusiki "r" eta "d" kontsonanteen galtzearen ondorio badira ere badira bestelakoak ere.

- r > Ø: bihamoun vs. biharamoun, bihéramounn, biharamun...; buia, buiac, büria, bouria

- d > Ø: bïaiantek, biayant, biayanteï, bidaïant, bidéiant, bidageant

- a > : bihâmun, biharamen 
- ch / s / z: bichtatic, bistatik, biztatik

- $\mathrm{u}(\mathrm{r}) \mathrm{u}>\mathrm{u}$ : buruz, buus, buuz, buz, bus

e) Grafia alternantziak

Ugaria da, zerbait esatekotan, grafia desberdinen agerrera; horietan ohikoenen artean c / $k$ grafiena da. Ondoan agertzen diren kasuak bakarño batzuk baino ez dira:

- r /rr: beharetan / beharretan, alhorak / alhorrac

- in / iñ / gn: artzainez, arçaïnez, arçagna / artzaïna

- s / z: élhourès, élhouréz...

- c/ ç / z: beçala, becala, bezala

- ou / u: bourouann, bourouia, buru-buruan, buruik

Lematizaziorako erabili diren irizpideak ondokoak dira:

1. Hitz baten aldaera guztiak lema berean sartzea izan da lehen irizpidea. Horrela agertuko dira escapatcen eta bere aldaera grafikoak, zein espacatcen eta bereak, denak lema berean.

2. Hitzaren aldaera guztiak lema berean baina ez sinonimoak, bistan denez. Horrela estali eta tapatu bi lematan agertuko dira.

3. Lemarako aldaera aukeratzean Hiztegi Batuko hitza denean horretan agertzen den aldaerari eman zaio lehentasuna. Hiztegi Batuan agertzen ez direnetan Orotariko Euskal Hiztegiari egin zaio kasu. Azken honetan ere ez bada agertzen forma osoenari eman zaio lehentasuna.

4. Kategoria gramatikal desberdinetako hitz homomorfoak bi lematan ezarri dira: behar 1, behar 2, kasurako.

5. Adizkiarekin batera agertzen diren partikulak, loturik agertzen izan direnetan; ez dira kontuan hartuak izan; hala nola beitcen, bainiz, bada, ezta... Halakoetan adizkiari eman zaio lehentasuna. Hala ere bereiz edo banandurik idatzita aurkitzen direnetan badute euren lema.

Ondorioz, bi testuetako hitzak kontuan izanik, 947 lema lortu dira. 


\subsection{Hiztegia eta adizkitegia}

Dokumentuetan zehar agertzen diren hitzak hiztegian bildu ditugu; halaber adizki guztiak adiztegian. Halarik ere, hiztegia ez dugu adizkiez hustu. Hauen zerrenda agertzen da lema nagusi batzuetan bildurik. Aditz laguntzailearen kasuan sei lema nagusitan bildu dira adizki guztiak: izan, izan NN, edun NN, edun NNN, edin, ezan NN eta ezan NNN.

Hiztegiaren ezaugarriak ondokoak dira:

a) Sarrera-hitza: aldaera edo testu-hitz guztien lema;

b) agerrera-kopurua: sarrera-hitzari dagozkion testu-hitz guztien kopurua;

c) aldaerak alfabetikoki eratuta;

d) aldaera bakoitza agertzen den dokumentuari dagokion herriaren kodea.

aberats (293): abaats (bes, hel, gab, hel), abaatsac (aih, gab), abaatsak (bas, urk, bas, urk), abarats (d-g, u-g, u-g), abaratsac (ezt), abaratxac (ban), abarax (ure, ure), abats (bes), abâtsac (bai), abatsac (bir), abatsak (bard), abatz (bard), abeassak (leko), abéatch (iito), abeatchac (aha), abéatj (iba), abéatjac (iba), abeats (d-b), abéats (etx, g-z, etx, o-z, ath, g-z, laka, l-z, l-z, men, o-z, ath), abeatsak (azka, gam, ilh, l-b, ilh, l-b), abeatx (a-m, ida, osp), abéatx (hau, hau), abeatz (altz, garr, garr), abéatz (alt, alt, altz), abeax (pag, izu, pag), abeaxac (izu, a-zo, g-zo, g-zo), Aberac (mau), abérach (ito), Aberas (aih), aberas (mar, uzt), aberasac (uzt), aberassac (azo), abératçac (hen), aberatch (sen, sen), abérats (ain, irur, kan, bun, don, don, zar, zar, bun), aberats (arn, beho, azka, bar, bid, bir, buz, esk, etxa, gam, hal, iho, iru, alo, izt, jat, jats, jut, lig, lan, larr, mak, mar, men, s-g, d-l, mug, ora, ort, s-g, d-l, aiz, d-g, d-i, u-h, arh, a-s, bar, zal, bai, suh, u-h, azk, zal, suh, zib, d-b, zib, esk, etxa, ida, iho, alo, jats, laka, lig, lan, larr, bida), Aberats (esp, hir, meh), Aberatsac (arm), abératsac (arm, ain, kan, l-s), aberatsac (arn, bid, lek, get, hal, iru, izp, izt, its, jat, jut, las, ald, luh, mak, leko, dono, haz, mend, ort, dono, las, ald, lek, iri, izp), aberatsak (bida, meh, zur, buz, ahe, esp, ezt, haz, zur), Abératss (bit), abératssak (bit), abératx (ainh, ainh, lekb, a-z, ost), ost), aberatx (arü, mit, dona, arb, urr, arr, arü, ürr, b-l, sarr, ünd, lekb, urd, osp, ürr, sarr, ünd), Aberatxac (dom), aberatxac (ora, d-i, dona, dom, arb, urr, arr, azk, b-l, ban), abératz (aint, beh, hen, l-s), aberatz (aiz, d-s, d-s, ahe, get, hoz, its, ame, l-o, l-o, bil, irur, ur-p, arh, a-s), aberatzac (hoz, ame), abératzak (beh), aberatzak (hir, mil, mil), aberax (l-a, luk, be-l, soh, gar), eih, lak, larz, lar, l-a, luk, amo, mit, müs, anh, arro, mau, müs, urd, arro, soh, gar, lak, larz), abérax (a-z, z-p, z-p), Aberax (lar), aberaxac (amo, mug, anh, iri, lah), aberaxak (sar, sar), aberaz (mend), abérazac (ur-p), abetx (g-i, g-i), eberax (be-1), habeatchac (aha).

abere begirazale (3): abere beguiraçale (arü), abere begirazale (sarr, ünd)

abere zaintzale (2): Abere zaintzale (lek), abere zaintzale (haz)

aberezain (2): abere zainec (hal, jat), aberezain (dom)

abian (1): abian (dom)

abiatu (1): abiatu (zib)

abildura (1): Abilduran (luk) 
abisatu (38): Abisa (ainh, amo, arü, altz, bar, g-z, sarr, ünd, osp, l-a, mau, o-z, izu, pag, arro, s-g), Abisaïc (l-z, müs), abisailk (gar), abisaïn (gar), Abisadi (alt, alo, etxa, g-i, lig, larr, zal), abisatcen (ainh), Abisaï (müs, l-z), abisaïk (gar), Abiseic (esk), Abiseik (laka), Abizadi (hau, ath), habisa (mar), Abizaic (urd)

adindu (1): adinduac (ame)

aditu (45): Aditciarequin (eih), aintxiarequin (arr), aïntziarequin (arb), Aitciarekin (mug), Aitciarequin (bai), aïtouric (ald), aitziaakin (hel), Aitzia-akin (aih), Aditcéaréquinn (hen), Aditcéarekin (ain), Aditcearekilan (ort), aditcearekin (bida, lek), Aditcearekin (esp, sen, uzt), Aditcearequi (ure), aditcen (urr), Aditcen (get), aditciarekin (azk), Aditciarekin (mak), aditsearekin (ban), aditsen (luh), aditu (zib), adituric (lar), Aditurik (mil), aditzearekin (ahe, haz, d-l, sar), aditzian (meh), Aditzian (bid), aditziarékin (lah), Aditziarekin (hal, jat), aitceaekin (leko), aitcearekin (hir), aitciaekin (bas), Aitziakin (bes), aitzian (urk), Aitziaquin (bir), Aitziarekin (zur, dono), aytcearekin (anh), ayturic (ur-p)

ager (3): ager (d-s, hoz, mil)

ageri (83): agéri (ito), agercen (men), agertcen (l-b), agertzen (buz, d-b, ünd, esk, gar, lak, sar), agertziaren (iho), aguértcéco (ain), aguértcen (ost), aguer (kan, iri, izt, jats, izu, dono), Aguer (dom), aguercea (urr), aguerceco (ort), aguercen (zar, azk, bir, leko), agueri (garr, lar, osp, hir, zal), aguersian (bard), aguertcéco (hen), aguertcën (g-z), aguertcén (hau), aguertceco (las, meh, mend, zal), aguertcen (ainh, amo, anh, arb, arr, arü, g-i, hal, iba, ilh, jat, laka, larr, lar, osp, mak, ora, o-z, pag, arro, s-g, sen, dona, ure, bil, z-p), aguertciaren (jut), aguertzearen (ame), aguertzeko (azka), aguertzen (alo, arh, a-s, sarr, gam, iru, lah, larz, luk, bai, u-h), aguertziaan (hel), aguerzia (ur-p), arguetceco (its)

agertu (19): agertcen (eih), agertu (zib), agertuco (bes), aguertceco (beho), aguertcen (a-m), aguerth (beh), aguertu (iri, lig, l-s, osp, urd, s-g, ath, irur, bil, z-p), aguertuco (lan), aguertuko (ahe)

agitu (11): agitu (bar, be-l, ito), agitzen (bar), aguitu (altz, soh, g-i, laka, larr, l-a, s-g), aguert (arn)

agorril (1): Agorrilako (ald)

ahal (10): ahal (bit, lek, zib, garr, osp, l-a, hir, dom, suh, bil)

ahaltsu (1): ahaltsu (l-s)

ahamen (1): ahamen (uzt)

ahuspez (2): ahuspez (zib, hir)

ailor (1): ailhor (s-g)

aintzira (5): aintcira (iri), aintzira (hal, jat, mak, d-l)

aipatu (4): aiphatu (bida, zib, iho, d-l)

aire (1): airian (mar)

aise (2): aïsa (alt), aïze (garr)

aitaso-amaso (2): aitasso_amassouac (zar), aitaso amassouac (aintz)

aitzin (139): aïntcinian (aint), aitcinean (arn), aitcinian (a-m, eih), aitzinian (beho), etchaïtcinian (larr), aïcinéann (hen), aïntcinean (ain, sen), aintcinian (meh), aïntciniana (ost), aintzinian (zar, jats), aitcinéra (don), aitcinean (lek, mug), aitcinera (etxa), aitcinian (amo, a-z, arh, arm, beh, g-zo, etx, gab, l-z, müs, s-g, bai, dona, ure), aitciniann (hau), aitcinien (bard), aitcinin (arü, altz, sarr, ünd), aïtcitsun (mend), aïtsiarékin (kan), aitsinéra (l-s), aïtsiniala (irur), aitsinian (bun, kan), aitsinila (o- 
z), aïtssiniann (bit), aïtzinïan (gam), aïtzinéan (lah), aïtzinea (iba), aïtzinean (haz), aïtzineat (garr), aïtziniala (g-z), aïtzinian (buz, gar, hel, lekb), aïxinean (d-g), aïzinian (u-h), aincinila (men), aincira (uzt), aintcignean (bida), aintcina (d-i), aintcinean (bl, its, d-i, hir), aintcinian (anh, esp, ilh, l-b), aintsinean (luh, luh), aintsinian (ban), aintzinea (d-b), aintzinean (hal, iru, ort), aintzineât (zur), aintzinera (izp), aintzinian (hoz, jat, lak, dono), ainzinian (lan), aitcinean (get, izt, leko), aitcinerat (ald), aitciniala (osp), aitcinian (ainh, a-zo, azk, soh, g-i, ida, jut, las, mak, mar, ora, izu, suh, u-g, urr), aitcinila (mau, bil), aitcinin (arro), aitziñean (zib), aitzinean (ahe, ame, sar, mil), aitzineraino (iri), aitziniala (l-a), aitzinian (alo, azka, aih, bas, be-l, bid, bir, bes, d-s, d-s, ezt, iho, larz, l-o, luk, dom), aitzinin (ürr, bar, esk, laka, mit), antcinian (mend), aytcinian ( $z-p)$, aytzinean (a-s), hanzinian (ur-p)

akabantza (3): akabançan (aiz), akabanzat (ur-p), akanzan (bir)

akabatu (1): akabatcian (ath)

akulatu (1): akhulatiac (lan)

ala (3): ala (esp, osp), Ala (osp)

alde (65): aldé (ain, a-z), Aldé (ain, kan), aldéat (beh, bun, ost, aint), aldérat (zar, hen, izt, lah, z-p), alde (bida, etxa, hal, ida, jat), Alde (b-l, zib, hal, jat, d-l), aldean (zib), aldeat (bes, gam, iho, jats, larz, las, leko, ora, bai, urr), aldeât (zur), aldebat (lar), aldebatez (l-o), aldera (beho, esp, luh, sar), alderat (arm, azka, eih, zib, ezt, etxa, get, hal, haz, iru, its, jat, jut, lak, luk, meh, ort, d-l, d-i, dona, ure, ur-p, uzt), aldootaïc (pag)

alderdi (5): Alderdi (bid), alderdico (lekb), alderdiko (lekb), alderdiraat (d-g), alderdirat (u-g) aldi (24): aldi (g-z, lekb, ora), aldiann (bit), aldibates (lar, mar), aldis (l-a, z-p), aldiz (altz, b-l, bar, lek, zib, zib, d-b, hoz, hoz, iho, iri, lar, osp, d-l, uzt, bil)

alegeratu (1): alagueatuik (altz)

alegia (3): aleguia (laka, laka, zal)

algatze (2): algatzik (sarr, ünd)

alha (100): alan (ahe), alha (zar, ainh, ame, a-z, arb, a-zo, arr, arh, arm, aih, b-l, ban, bar, be-l,

bit, bid, bir, buz, kan, g-z, g-zo, soh, d-b, esk, etx, etxa, gar, garr, get, hal, hau, iba, iho, izt, jat, jats, lak, laka, lig, larr, l-s, osp, l-z, luk, mau, mug, müs, ora, urd, o-z, arro, d-i, hir, dono, bai, suh, ath, irur, ure, uzt, z-p), alhacélaric (ito), alhacen (g-i, mar, pag), alhan (ain, alt, alo, amo, anh, arü, ürr, lek, d-s, sarr, ünd, ida, iri, izp, lah, lar, ald, mit, d-g, sar, u-g, u-h, arn, eih), alha (aint), alhacen (a-m), alhancen (ilh, l-b), alhantzoon (larz), alhazen (a-s, dom), alhen (l-a, l-o, s-g)

alhatu (28): alatcen (meh, sen), alatzenzen (zur), alhatcen (aha, azk, bard, gab, hoz, jut, las, mak, men, ost, zal, dona, bil), alhatcencen (leko), alhatsen (bun), alhatzen (azka, bas, beho, bes, ezt, hel, iru, mil), allatcen (its), halatua (zib)

aloiatu (1): aloyatu (iho)

alor (75): alhôrrac (hau), alhor (ahe, altz), alhorac (g-z, etx, ida, lig, l-z, mar, mau, müs, o-z, bil, z-p), Alhorac (urd), alhorak (ürr, gar, l-o), alhorrac (aint, beho, zar, alt, ame, anh, arh, azka, ban, be-l, bit, bid, bun, soh, esk, ezt, g-i, hoz, iba, jut, laka, lan, larz, larr, l-s, las, osp, l-a, mend, ost, izu, pag, arro, s-g, d-i, zal, suh, ath, irur, u-h, ure), Alhorrac (ainh, arn, b-l, bai), alhorrak (a-s, bar, sarr, ünd, men, mit, don), Alhorrak (alo, d-s), alhorrer (izp), alhorreri (ald), alorrac (jats)

altaman (8): Altamann (beh, beh, beh, beh, beh, beh, beh), Altamanni (beh)

altxatu (17): alchatiac (ban), alchatou (hen), alchatu (anh, bida, bir, ezt, get, its, urr), alchatuac 
(ort), alsatun (ur-p), altchatu (ahe, bas, garr, sar), altchatuyak (zur), artzarcean (get) ama (177): ama (alt, alo, arb, a-zo, arr, arü, ürr, azka, bar, be-l, d-s, g-z, g-zo, soh, zib, d-b, ünd, esk, ezt, etx, etxa, gar, garr, garr, g-i, iho, ilh, ito, its, l-b, laka, l-s, osp, l-z, men, mit, urd, o-z, hir, ath, urk, ur-p, uzt, bil), amaï (hau, irur), ama'ri (ban), amac (aint, zar, ainh, ame, a-zo, aih, b-l, ban, bit, lek, g-z, g-zo, gab, hal, iri, ito, jat, lan, ald, müs, s-g, bai), amai (bid, bes), amak (aiz, ahe, altz, beh, bida, d-s, haz, d-l, mil), amarén (ain), amaren (bida, hal, hoz, jat, lar, sar), amari (a-m, aha, aint, aiz, arn, beho, eih, zar, ahe, ainh, amo, anh, a-z, arh, arm, a-s, azk, aih, beh, b-l, bard, bas, bit, bir, bun, buz, kan, sarr, esp, gab, gam, get, hel, haz, hen, iba, ida, iri, iru, izp, izt, jats, jut, lak, lekb, lig, lah, lan, larz, larr, las, ald, l-a, l-o, luh, luk, mak, mar, mau, meh, leko, mend, mug, müs, ora, ost, ort, izu, pag, arro, d-l, d-g, d-i, sen, don, zal, zur, dono, bai, dona, dom, suh, u-g, u-h, ure, urr, mil, z-p), amk (d-b)

aments (1): aments (gab)

amoina (1): amoïna (bit)

amoregatik (25): amoéagatic (ito), amokatik (bard), amoréakatik (aiz), amore gati (bas), amoreacatic (be-l, soh), amoreagatic (ame, b-l, larz, ora, ure), amorea-gatic (sen), amorecatic (bid, l-a, mar), amoregatic (u-g), amoregatik (hoz), amorekatic (d-g), amorekatik (a-s, l-o, bil), amourecatic (arü, larr), amourekati (ürr, mit)

amorratu (2): amurratuez (ur-p), amurratuz (bir)

andre (7): andre (ort, ort), andrea (ort, sen), andria (bir, ur-p), anndréa (hen) añik eta (6): agniketa (arro, etx), agniceta (ida), agnic_eta (larz), agniketa (1-z), agniqueta (1-a) anitz (157): ainhitzetan (ame), anhichco (jut), Anhichko (ilh), Anhits (bit), anhitsetan (bard), anhitz (arm, a-s, bard, bit, iba, jats, luk), Anhitz (a-z, bard, buz, gam, hoz, izp, l-b), anitz (zar, azka), Anitz (bes, ezt), aniz (aint), haïnitcetan (zar), haïnitcétan (aint), haïnitz (arn, beho, ain, lah, meh, sen, sen), haiñitz (zib), hainitch (osp), hainits (esp, mak), hainitz (ame, ame, lek, get, get, iri, its), hainitz eri (ald), hainitz (ald, d-i, hir, hir, zur, urr, urr), Hainitz (lek, hal, jat, las, sar, dono, uzt), hanic (irur), hanich (alt, arü, etxa, etxa, ida), Hanich (alt, lig), hanichco (amo), Hanichco (iho), hanichcotan (l-s), Hanichko (arü), hanitch (alo, alo, altz, altz, altz, bar, be-l, be-l, g-z, g-z, soh, sarr, ünd, esk, etx, hau, osp, l-z, l-a, mau, mau, men, men, müs, urd, o-z, pag, s-g, zal, zal, ath, ath, bil, bil, bil), Hanitch (bar, laka, urd), hanitchetan (men), Hanitchko (sarr, ünd), hanits (alt, bun, ida, mar, mend, suh, irur), Hanits (ban, kan, lekb, o-z), hanitsetan (a-zo), hanitx (alo), hanitz (ainh, ürr, ürr, azk, azk, aih, bas, bida, g-zo, soh, g-i, haz, haz, iru, lak, larr, l-o, luh, luh, leko, mit, mit, müs, ora, izu, s-g, dona, z-p, z-p), Hanitz (ainh, bid, g-i, haz, dom, urk, z-p), hanix (gar, gar, arro, arro)

anixko (9): hanichco (amo), Hanichco (iho), hanichcotan (l-s), Hanichko (arü), Hanitchko (sarr, ünd), hanizko (garr, garr)

apal (1): aphalaguac (ban)

apalgune (1): aphalgune (izt)

aperzebitu (23): aperbecitu (men), aperceittou (ost), apercebitcen (u-g), apercebitu (arh, altz, be-l, g-z, soh, sarr, ünd, hau, jut, mend, ath, u-h, z-p), aperceguitu (bir), aperceitu (bard), apercibitcen (d-g), aperzebitzendizi (gam), aperzeitu (urk), aperzeitzendu (aiz), aprecebitu (izu)

$\operatorname{aran}(1)$ : arana (bai)

arazi (285): egonasten (izu), egoneazten (laka), ekhararasten (b-l), ekarazten (anh), 
Ekharraaazten (hel), Ekharraassico (bun), ekharrarazteko (mil, ezt), Ekharrazico (bas), ekharrazteco (hir), Ekarrazten (aih), ekharrazten (buz), erdiaraztéaïnocoan (az), erdiazteko (müs, gar), erdierazteko (soh), erdikatzeko (mil), érdiraastéco (ost), erdiraazteko (d-b), erdirasteco (d-i), erdirazteco (esk), geldi-arazten (sar), guibelarazten (iru), iarraaci (d-g, d-g), iarraraci (ito), jar-aci (arü), jar_éaci (alt), jar_eraci (mau), jar_aci (men), jar_erassi (mit), jar_eraci (müs), jar_araci (ora), jareraci (urd), jar_araci (ost), jar_eaci (pag), jar_eraci (arro, s-g), jar_erazi (zal), jaréraci (z-p), jar_arasi (arh), jar_errassi (ürr), jar_erazi (bar), jar_eraci (be-l), jararasi (kan), jar_araci (soh), jar_aci (ünd), jar_erazi (esk), jar_éaci (etx), jar_eraci (etxa), jar_erazi (gar), jar_eraci (g-i), jar_ haraci (hen), jar_eraci (ida), jar_arazi (iho), jar_araci (iru), jar_arrazi (izp), jar_erazi (alo), jar_arazi (lak), jar_eraci (larr), jar_eaci (osp, l-z), jar_eraci (mar), jar_eaci (bil), jaraci (g-z, hau), jarra'asi (ban), jarraazi (d-b), jarraci (altz), jarrarasten (lar), jarraraci (d-i), jarrarazi (u-g, mil, d-s), jarréraci (ainh), jarreraci (laka), Jinaracico (d-i), jsara'asi (ban), sararasten (luh), sar_eraci (mau), sar_aaci (leko), sar_aci (men), sar_eaci (mit), sar_eraci (müs, amo), sar_araci (ora), sareaci (pag), sar_eraci (arro, s-g), sararazi (d1), sar_aci (anh), sar_eraci (zal), sar_arazi (bai), sar_araci (dona), sar_erazi (dom), saréaci (ath), sar_arasi (u-h), sar_araci (urr), saréraci (z-p), sararazi (arr), sar_araci (arh), sar_errassi (ürr), sar_arazi (a-s), sararaci (azk), sar_arazi (aih), sarhaaci (beh), sar_eraci (b-l), sar_araci (ain), sar_erazi (bar), sar_araci (be-l), sar_arasi (kan), sar_ eraci (g-z), sar_araci (soh), sarharazi (zib), sar_erazi (esk), sar_éaci (etx), sar_araci (etxa), sar_erazi (gar), sar_eraci (g-i), sararaci (get), sar_eraci (ida), sar_araci (iru), sar_arazi (lak), sar_erazi (lekb), sar_araci (lah), sar_eraci (larr), sar_harasten (ald), sar_ eraci (osp), sar_eaci (l-z), sar_eraci (l-a, mak), sareraci (mar), sarassi (l-s), saraci (izu), sarrâzi (zur), sarazi (urk), saraci (gab, hau), sarraasten (a-zo), sarraatci (mend), sarraazi (azka, bas, bes, d-b, gam, hel, jut, larz), sarraazten (g-zo), sarraci (ilh, l-b), sarrarazi (meh), sarraraci (mug), sarraraçi (ort), sarraraci (d-i), sarraraztén (don), sarraraci (hir), sarrarazi (dono), sarraraci (a-z, suh), sarrarazi (u-g, arb), sarraraci (ure), sarrarazi (mil, bid), sarraaci (zar), sarraraci (lek), sarrarazi (buz), sarrarazten (ahe), sarraraci (esp), sarrarazi (ezt), sarréraci (ainh), sarrarazi (hal), sarraraci (hoz), sarrarazi (izp), sarrarasi (izt), sarraraci (its), sarrarazi (jats), sarreraci (laka), sarraaci (las), sarrasteco (altz), sarrasten (lan), sarreci (bard, bard, bir), sarrhassi (bun), ssar_araçi (bit), ttoto_eraci (l-a), ttottoaci (ath), urruntaraziac (izp), chararazi (sen), yar_eraci (amo), yar_arassi (mend), yar_araci (ost), yararaci (sen), yar_arazi (bai), yar_araci (dona), yar_erazi (dom), yar_arasi (u-h), yar_araci (urr, uzt), yar_arazi (a-s), yar_araci (ain), yararaci (azk), yarhaaci (beh), yar_eraci (b-l), yar_araçi (bit), yar_aasten (g-zo), yar_harazi (zib), yararaci (jut), yar_arazi (ame), yar_erazi (lekb), yar_arazi (lah), yar_eraci (), yar_ harasten (ald), yar_eraci (mak), yarraasten (a-zo), yarraàzi (azka), yarraazi (bas, bes, gam, hel), yarraci (anh, izu, ur-p), Yarraci (arm), yarraci (bir, gab, ilh, l-b), yarrarazi (meh), yarraaci (leko), yarraraci (mug), yarraraçi (ort), yarrarazi (d-l), yarraraci (a-z), yarraraztén (don), yarraraci (hir), yarrarazi (dono), yarraraci (suh, ure), yarrarazi (arb, arr, aih, bid), yarraraci (lek), yarraaci (zar), yarrarazi (buz), yarrarazten (ahe), yarraraci (esp), yarrarazi (ezt, hal), yarraraci (hoz), yarraaci (iba, iba), yarrarasi (izt), yarraraci (its), yarrarazi (jat, jats), yarraaci (las), yarrazten (lan), yarreci (bard), yarrhassi (bun), zalazi (garr), çareraci (urd), çar_haraci (hen), zar_arazi (ame), zarrazi (garr), zarrarazi (d-s, jat), çarri (ath), zartarazteraïnokoa (ahe), zartasteco (izu), sar éassi (lig, lig, o-z, 
o-z, irur) jar éassi (irur), yar arasten (luh), alha azi (bar), sar hazi (haz), yar erasi (l-o), jin erasten (etxa)

arbaila (7): arbailla (ürr, men, mit, arro, zal, bil), arbailla (hau)

ardi (22): ardi (a-m, aha, ahe, arn, azk, bun, g-z, d-b, hel, its, luh, mak, mug, sen, uzt), ardia (beho), arditic (meh), ardizko (bida)

ardialde (1): ardi aldebat (lar)

ardimultso (6): ardi molcho (a-z), ardi moulço (arm), ardi moultzo (ora), ardi mulcho (luk), ardi multsu (esp), ardi multçu (ure)

ardioste (1): ardi oste (urr)

ardisaldo (43): ardi-saldo (ürr), ardi saldo (ainh, altz, amo, arro, ath, bar, bil, bun, dona, d-i, d-s, esk, etx, gam, g-i, hau, hoz, ida, irur, izp, izu, laka, larr, l-a, l-z, lig, men, müs, ort, o-z, s-g, sarr, ünd, zal), ardi saldoat (jats), ardi saldobat (alo, arü, gar, l-s, l-o, mit, pag) arditropa (48): arditropabat (mar), ardi tropa (aih, ame, a-s, azka, be-l, bit, buz, dono, etxa, ezt, gab, get, g-z, hen, iru, its, ito, izt, jut, lah, lan, las, lekb, luh, mau, meh, ost, soh, suh, zur), ardi tropat (bard, bes, urk), ardi tropat (bir, garr, ilh, l-b), ardi tropaat (beh, g-zo, mend), ardi tropa'at (ban), ardi tropabat (a-zo, altz, b-l, mil, z-p), hardi tropa (ur-p)

ardura (44): ardoura (aha, ain, arm, eih, gam, hel, iba, jut, lekb, ald, dona), ardura (ainh, anh, a-z, arn, azka, beh, beho, b-l, ban, bit, bid, d-s, gab, hoz, iho, iru, izp, izt, jats, lak, lan, larz, las, leko, mend, urd, d-g, d-i, zal, bai, suh, irur, u-g)

argi (25): argiz (bar), argui (ahe, arh, bun, gar, g-i, iri, lekb, don, zal), argui (aint, eih), arguia (aint, arh, eih), arguibates (alt), arguiric (a-m), arguis (mar, z-p), arguitan (jut), arguiz (ame, lan, luk), arguitouic (aha), arguizko (garr)

argi-argia (38): $\operatorname{argi}$ argia (ürr, sarr, ünd, men, mit, ito, l-b, l-o, u-h, esk), argui_arguia (iba, larz, izu, ainh, osp, bil, azka, las, zar, ezt, mend, aiz, a-zo, arü, beh, g-zo, etxa, ilh, alo, g-z, hau, laka, l-a, o-z, ath), argui-arguia (ain, amo)

argidura (2): arguidua (urd), arguidura (arro)

argitu (11): argitzen (urk), arguitcen (ida, pag), arguitia (l-s, ald, ost), arguitua (lek, urr), arguituic (müs), arguituric (larr, mau)

ari (201): aïçûtutcen (alt), aïçuçun (alt), aïcén (hau), aïcen (pag), aicitçun (mar), aïzen (hel), ari (a-m, aint, arn, beho, eih, zar, zar, ainh, ainh, ame, ame, amo, amo, anh, a-z, a-z, a-zo, arr, arh, arh, arm, arü, arü, ürr, ürr, a-s, b-l, ban, bar, bard, bas, be-l, be-l, bit, bit, bit, bid, bes, bes, bun, d-s, buz, buz, buz, kan, soh, soh, sarr, sarr, d-b, ünd, ünd, esk, esk, ezt, ezt, ezt, ezt, etxa, etxa, gam, g-i, hoz, iba, iba, iba, ilh, ilh, iri, iru, iru, izp, izp, izt, ito, jats, jats, jut, l-b, lak, laka, laka, lekb, lekb, lah, lah, lan, larz, larr, l-s, l-s, las, las, osp, l-a, l-a, l-a, l-o, l-o, luk, mak, mak, mak, mau, mend, mit, mit, müs, ora, urd, ost, ort, arro, arro, arro, s-g, d-g, bai, bai, dona, dom, dom, suh, suh, u-g, u-g, u-h, u-h, bil, bil, bil), aricen (d-g), arician (ure), aricien (l-b), aricirelaric (mar), ari_cirelaric (iri, las, s-g), arizen (aiz, a-s), arizien (azka), arizirelaik (azka), ariziren (aiz), arizutuzun (esk), haï (hen), haïcen (mug), haïciren (mug), haicen (esp, hir), haiciren (esp), haizen (urk), haizielaik (zur), haizuin (zur), hari (ain, ain, ahe, arb, arb, arr, beh, beh, bida, bida, lek, kan, get, hal, haz, hen, hen, its, its, jat, lah, luh, meh, meh, d-l, sen, sar, sar, urr, urr, uzt, uzt), haricen (dono), haricirelaric (hir), hari_cirelaric (ald)

aritu (1): haitu (ahe)

arraildu (39): arailtzeco (dom), arailatzen (zib, d-l), arrailatceco (uzt), arrailatzeco (iru), 
arrailatzeko (hel), arrailceco (a-m, leko), arrailcecua (arn), arrailcecoa (ort), arraildu (get), arrailtcéco (ain), arrailtceco (sen), arrailtceko (arb, arr), arrailttcerainocoan (dona, suh), arrailtzeco (aha), arrailltzeko (ora, bai), arrailzeko (jats), arraillaceco (bir), arraillatcéco (hen), arrailtatceco (urr), arrailtatzecoa (l-o), arrailtceco (amo, b-l, bida), arrailtceko (las), arrailtceraino (esp), arrailtcerainocouan (jut), arrailtseco (ban), arrailtzeco (iri, dono), arrailtzeko (gam), arrailtzeraino (hal, jat), arraitçeko (ur-p)

arrailgarri (1): arailagaria (azk)

arrailki (1): arraillki (mug)

arrangura (9): arrangourac (arm), arrangurac (aih, ort, dono), arrangurak (esp), errenkurac (bir), errenkurak (arb, arr, bida)

arranguratu (1): Arainkuratu (bar)

arrapru (2): arraproua (g-i, g-i)

arras (51): araz (anh), arrajs (ban, ban), arras (ain, a-m, arn, a-z, arb, arr, aih, bid, bid, bida, bir, bes, bun, d-s, zib, esp, gab, haz, haz, hen, hoz, hoz, iho, iru, izp, jats, jut, lar, ora, ort, d-l, don, hir, sar, zur, dono, bai, suh, urk, ur-p, urr, uzt, mil), arrass (bit), arraz (ahe, its, d-i), arratz (ald)

arrastatu (14): arastatou (luh), arastatu (müs), arestatu (g-z, l-s), arrastatu (azka, haz, its, d-g), arratztatu (mil), arrechtatou (aha), arrestatu (mit, z-p), arreztatu (ürr, garr)

arrasti (12): arasti (g-z, hau, o-z), aresti (bil), aristi (etx, l-z, l-a, mau, urd), arrasti (alt, men), arristi (ath)

arrastiri (29): arastiri (amo), arastiribatez (ida), Arestiri (sarr, ünd), aristiibates (irur), aristiri (arü, müs), aristiribatez (pag, z-p), ariztiibatez (lig), arrastiri (be-l, soh, esk, zal), arrastiry (s-g), arratsiri (d-g), arraztiri (ürr), arrestiri (ainh, laka, mit), arristi (altz), arristiri (bar, gar, g-i, osp, arro), Astirian (ilh, iri)

arratsalde (81): aatsalde (bir, hel), aatsaldeetez (urk), Uztaillaco-atjalde (iba), arats aldé (ain), arats aldebatez (l-o), arats aldi (ora), aratsaldé (kan), aratsalde (zib, luh, d-l, mil), aratz alde (etxa), aratxalde (azk, dom), arax aldibates (mar), araxalde (lak), arratçalde (its), arratch-alde (sen), arrats alde (bida, hal, jat), Arrast apalean (ahe), arratsalde (ahe, lek, buz, haz, iho, iri, izp, luk, leko, ort, uzt), arratsaldebates (esp), arratsaldebatez (hir), arratxalde (arb, arr, lah, d-i), Arratzalde (ame), arrax aldi (lekb), arrax aldibates (lar) arrax-alde (sar), Arraxalde (hoz), arraxaldi (mak), asalde (ur-p), atchalde (aha), atjsalde (ban), atsaldé (a-zo, bun, l-s, don), atsalde (azka, bas, bid, bes, g-zo, d-b, ezt, gab, gam, iru, ald, bai), âtsalde (zur), atxaldé (ost), atxalde (dona), atzalde (arh, d-s, u-h), Atzalde (aiz), atzaldeatez (garr), axaldé (a-z), axalde (anh, aih, b-l, lan, larz, izu, ure)

arren (5): arren (bir, bir, urd, ur-p, urr)

arres (2): arez (urd), arres (osp)

arribatu (3): arribatu (garr, zur), Arribatun (ur-p)

arroka (117): aroca (a-m, g-z, ida, l-s, bil), aroka (zib, men, mit, urd), arroca (ainh, arü, be-l, bir, lek, soh, etxa, hen, jut, lah, lan, ald, mau, ora, ost, d-i, sen, hir, ur-p), arrocabat (bun), arrocabaten (z-p), arrocâten (mug), arroka (a-zo, ürr, a-s, altz, beh, bar, g-zo, sarr, ünd, ilh, jats, ort, dono), arrokaaten (urk), arrokaten (aiz, l-b), arrokatzutan (garr), haroca (azk, lak), haroka (kan, müs, d-l), harokan (alo), harroca (ain, aint, arn, beho, eih, zar, ame, anh, arm, aih, b-l, bas, bit, bes, ezt, get, hel, hal, hoz, iba, iho, iri, izp, izt, ito, its, jat, larz, las, luk, mar, leko, o-z, bai, dona, dom, suh, u-g, urr, uzt), harrocac (anh), harrocaten (mend), harroka (ahe, a-z, arb, arr, bida, d-s, buz, d-b, esp, gam, haz, 
lar, mak, meh, don, zur, mil), harrokaten (bard, gab), harroquen (g-zo), harroska (sar) $\operatorname{arrotz}(65)$ : $\operatorname{arotcec}($ azk, etx, pag), arotcek (l-z), arotsec (irur), arotsek (lig, o-z), arotzec (l-a), arotzek (lak, mit, urd), arrotçec (ure), arrotcéc (ain), arrotcec (aint, arn, a-z, g-i, izt, lar, meh, ort, s-g), arrotcek (zar, mau, ath), arrots (ban, esp, esp), arrotsac (ito), arrotséc (ito), arrotsec (alt), arrotz (aint, zar, bas, haz, lar, d-l, sen, hir, hir, sar), Arrotz (lek, haz, haz), arrotzak (d-l), arrotzec (b-l, lek, bes, ezt, hel, hal, hoz, iri, izp, jat, mug, arro, zal, bai, uzt), arrotzek (ahe, ürr, d-b, gar, leko)

artalde (32): arthaldibat (eih), ardi arthalde (mug, mak, azk, ahe, sen, uzt, hel, dom), ardizko arthalde (bida), artalde (bid, lek, leko), arthaldé (ain), arthaldébat (kan), arthalde (arb, arr, bas, d-b, hal, haz, iho, iri, jat, lak, larz, ald, d-l, sar, bai), arthaldea (zib), arthaldebat (hir)

artatzaile (1): arthaçaleric (lar)

arte (189): isanarté (ur-p), arté (ost), artéan (aiz, ain, ain), artéänn (hen), artin (g-z), arte (arm, azk, bard, bard, bida, bir, lek, zib, esp, ezt, get, get, haz, hoz, iru, larz, dono, urk, ure), artean (ahe, ame, ame, a-s, beho, b-l, bida, lek, zib, get, haz, izt, izt, its, its, lak, meh, leko, mug, ort, d-l, d-i, hir, sar, dono, ure, uzt, mil), artebatetako (a-s), arteco (z-p), arteraïnokoan (ahe), arteraino (iri, hir, urr), artetik (luh), arthe (meh), artia (l-b), artian (a-m, aha, aint, arn, beho, eih, zar, ainh, alt, alo, amo, anh, a-z, arb, a-zo, a-zo, arr, arh, arm, arü, azk, azka, aih, aih, beh, ban, bas, be-l, bid, bun, d-s, buz, buz, kan, g-zo, soh, d-b, esp, ezt, etx, etxa, gab, gam, gar, garr, g-i, hel, hoz, iba, ida, iho, ilh, iri, iru, izp, ito, jats, jut, l-b, lekb, lig, lah, lah, lan, larz, larr, lar, l-s, las, ald, osp, l-z, l-a, l-o, luk, mak, mak, mar, mau, men, mend, müs, ora, ora, o-z, izu, pag, s-g, d-g, sen, sen, don, zal, zur, bai, dona, dom, suh, ath, irur, u-g, u-h, urr, z-p), artiane (ost, ost), artiann (bit, hau), artien (bard), artin (ürr, altz, bar, bes, bes, sarr, ünd, esk, laka, mit, urd, arro, urk, bil), artiraino (d-g), hartian (arh)

arteka (5): erdiartekan (l-b), éartekan (irur), ararteka (a-s), artekan (zal), eartekan (lig) artio (107): bilartio (urd, ainh), erdiartino (hau), erdiastekan (ilh), eçartio (mar), içanartio (mit), sarartino (alt), sarrartio (jats), artino (alo, amo, arh, arm, arü, altz, bar, be-l, lek, bun, g-z, g-zo, soh, sarr, ünd, esk, etxa, gar, garr, g-i, hel, hau, ida, lah, lan, larr, ald, l-a, luk, mau, men, müs, o-z, pag, s-g, d-i, don, suh, mil, z-p), artio (aiz, a-m, aha, aint, arn, eih, zar, anh, a-z, arb, arr, ürr, azka, beh, b-l, ban, bas, bit, bid, d-s, kan, d-b, ezt, etx, gab, gam, hal, haz, hen, hoz, iba, iho, ilh, izp, ito, jat, jut, lak, laka, larz, las, osp, l-z, luh, leko, mend, mug, ort, izu, arro, zur, bai, dona, dom, ath, u-g, u-h, uzt)

artzain (257): artçaïna (a-m, aha, aint), artçaina (eih), artçaines (aha), artçañez (a-m), artzaina (arn, beho), artzainez (arn, beho), arçainez (mug), arçagna (a-z, a-zo, g-zo, lan, men), arçagnac (bit), arçagnez (bard, men), arçaign (g-zo), arçaigna (soh), Arçaigna (mau), arçaignez (lan), arçain (a-z, a-zo), arçaina (azk, urr), Arçaina (ort), arçainez (azk, ort, urr), arcaïna (arm), arcagna (mar), arsagna (bard, ito, ito), arsagnés (ito), arsaignez (ban), arshaïna (bun), artçaïn (ida), artçaïna (ain, zar, anh, arü, be-l, hen, las, mug, ure), Artçainna (sen), Artçainétaz (ain), artçaïnez (arm, ure), artçagn (altz), artçagna (ainh, alt, amo, altz, gab, g-i, ida, ilh, l-b, laka, larr, l-a, müs, ora, izu, pag, arro, s-g, zal, ath, bil), Artçagna (etx, hau, l-z), artçagnès (bit, hau), artçagnec (alt), artçagnes (amo), artçagnez (soh, etx, gab, g-i, iba, ilh, laka, larr, l-z, l-a, mar, müs, pag, arro, s-g, zal), artçaiñ (osp), artçaiña (osp, urd), artçaigna (beh, etxa, jut), Artçaigna (bida), artçaignetcaz (etxa), artçaignez (beh), artçain (esp, its, l-b, urd, d-g, don, hir, suh), artçaina (b-l, esp, izt, its, 
lar, mak, leko, d-g, d-i, don, suh, z-p), Artçaina (hir), artçainec (lar), artçaines (ald), artçainez (anh, b-l, hen, las, mak, d-i, z-p), artaiñ (zib), artazagna (mit), artcagna (ost), artcain (bida), artcaina (ald), artsaina (kan), artsaïnéz (kan), artsagna (g-z, lig, l-s, irur), artsain (luh, u-g), artsaina (ban, luh, mend, o-z, u-g), artsainez (dono), artxaina (arr), artxainez (arr), artzaña (ürr, d-b, ünd, esk), artzaïña (sarr), artzaïez (dona), artzaïn (gam, lah), artzaina (ahe, arb, azka, lek, buz, hel, haz, iru, jats, lekb, lah, meh, bai, dona), artzaines (arb, arh), artzaïnez (ahe, buz, iru, jats, lekb, meh, bai), artzagna (alo, gam, gar, garr, iba, larz, l-o), artzagneke (garr), artzagnetzaz (gar), artzagnez (be-l, larz, mit), artzaiñ (bar, zib), artzaiña (bar), artzain (ame, aih, bes, esk, garr, hoz, d-1), artzaina (ame, aih, bas, bes, d-s, ezt, get, hal, hoz, iho, iri, izp, jat, lak, d-l, sar, zur, dono, dom), Artzainac (ezt), artzainak (urk), artzainetaz (lak), artzainez (bas, hel, izp, mil), artzeina (mil), arzaïna (aiz, luk, $\mathrm{u}-\mathrm{h}$ ), arzaïnes ( $\mathrm{u}-\mathrm{h}$ ), arzaïnetaïk (aiz), arzaïnez (luk), arzaina (arh, a-s, bid, bir, uzt), Arzaina (ur-p), arzaines (bir, ur-p), arzainez (bid), arzaynez (a-s), asaïnez (mend)

asaldatu (2): achaldatiac (ito), assaldatuez (bida)

ase (1): azeazi (garr)

aski (4): aski (zib, esk, osp, arro)

asko (20): achkori (sen), asco (anh, bir, eih, iri, mug, ort), Asco (b-l, lar), ascotan (kan), asko

(ahe, hel, urd, ure), Asko (arb, arr, d-l, mil), Asko (ahe), askotan (hoz)

asmatu (1): asmatu (arro)

aspaldi (1): aspaldi (aih)

aspre (1): asprea (ahe)

ate (68): até (ur-p), atéa (hen), ate (bir, esp, haz, hen), atean (mak), atearen (haz), atez (bard), athé (ain), athéaren (ain), athe (ahe, arb, arn, arr, azk, bida, zib, get, hal, jat, lar, meh, ort, d-l, sen, hir, hir, sar, zur, zur, bai, ure, urr, uzt), Athe (bida), athean (lar), athearen (ahe, arn, bida, zib, hal, jat, lar, meh, ort, d-l, sen, hir, sar, ure, uzt), athera (d-l), atheri (ahe), atheric (hir), athiaîn (bai), athiaren (arb, arr, azk, ban, get, ald, zur, urr), atia (ur-p), atian (bir, izu), atiaren (esp)

atera (16): atheatu (bas), athera (azk, b-l, ban, ezt, hal, hoz, jat, bai, urr), atheratcean (hir), atheratcen (arm, urr), atheratu (lek), atheratuko (d-l), atheratzen (zib)

ats (1): Atsarribuz* (1-b)

atso (1): atsoat (bard)

atxiki (59): achikasten (ur-p), achiquicen (bir), achiten (bard), atchiitzen (hel), atchikarasten (dono), atchikitcén (ost), atchikitcen (zar, anh, a-z, arm, azk, jut, l-b, las, mak, mar, dona, dom), atchikitcendu (ort), atchikitcentci (iba), atchikitcentu (leko, mug), atchikiten (ilh), atchikitsen (ald), atchikitzen (arh, arn, beho, bid, buz, zib, zib, ezt, lak, lekb, lah, larz, d-l, u-h, urk, mil), atchikitzentiztu (aiz), atchikitzentou (aha), atchikitzentu (azka, zur), atchikitzentzi (gam), atchiquitcen (amo, eih, get, d-i, ure), atchiquitecen (mend), atzikitzentzi (garr), etchekitcen (s-g, arü), etchekitzen (sarr, ünd, gar), etchekitcen (osp)

atzarri (22): atçarciarekin (azk), atçaria (mar), atçarria (bida, lar, sen, hir), atçarriric (arü), atçartcéarékin (ain), atçartcean (its), atçartcearekin (sen), atçartzean (hir), atciarra (ort), atxarria (arr), Atzarri (d-l), atzarria (arb, zib, get, d-l), atzartzean (sar), atzartzearekin (lek), atzartzen (zib), atzartziarekin (haz)

atzeman (8): atzemaiten (beh), hatcemaiten (l-b), hatcemaiten (ilh, izu), hatchematen (get), 
hatséman (l-s), hatzamaiten (urk), hotçamanik (ath)

auhen (4): aühenac (ure), auhenac (lar, uzt), auhenak (sar)

auhen-min (1): hauen-miñak (zib)

aurkitu (16): aourkhitcen (aint, zar), aourkitcen (sen), aourkitou (sen), aurkhitcen (hal, its,

jat), aurkhitu (ahe, b-l, hal, its, jat), aurkhitzen (zib, zib, zib), aurkitu (zib)

ausartu (1): aousartatsén (1-s)

auzo (146): aïçoac (bit, ito), aïçouac (ainh, amo, a-z, g-zo, soh, etxa, gab, hau, l-z, müs, ora,

pag, s-g), aịçouk (arü, altz), aïcouac (g-z, etx, g-i, ida), aïcouak (ath), aïsouc (ünd), aïssokouak (bard), aïssouac (lig, l-s, ost, irur), aïzouac (arh), aïzouak (alo, gar, garr), aïzouk (sarr), aïzuac (u-h), aïzuak (aiz), aiçouac (be-l, osp, l-a, urd, arro, zal), aiçouak (ilh, l-b), aiçouc (laka), aiçouk (men), aicouac (mar), aisouac (mau), aissouac (a-zo, o-z), aissouak (beh), aizüak (d-b), aizoac (ame), aizouac (esk), aizouk (ürr, bar, mit), aoüssouac (bun), aouçoac (lah), aouçouac (zar, iba, jut), aouçouak (don), aousouac (aha, aint, kan), aouzouac (hel, dona), aouzouak (buz, gam), aüçoac (ort, ure), aüçoak (mug), aüçuac (lar, d-g), aücoac (arm), aüssuac (luk), aüsuac (b-l, bas), aüzoac (bai), aüzoak (lek), aüzouac (lekb), aüzuac (jats, suh), auçoac (azk, iru, izt), auçuac (a-m, las), aucoac (anh), ausouac (eih), aussoac (mak, mend), ausuac (ban, izu, d-i), ausuak (l-o), auzoac (aih, bid, ezt, hal, izp, jat, lak, lan, dom), auzoak (leko, mil), auzuac (beho, bir, bes, hoz, iho, larz), auzuak (azka, u-g), auzuk (urk), ayçouac (larr), ayçouk (bil), ayssoac (z-p), ayzoak (a-s), ayzuac (ur-p), haüsoac (arr), haüzoac (arb, get), haüzoak (ahe, haz, meh), haöuçoac (hen), haouçoac (ain, sen), haousoac (ald), hauçoac (its, hir, urr), hauçoak (bida), hausoac (luh), hausoak (esp), hauzoac (iri, uzt), hauzoak (zib, sar), hauzuac (dono), hauzuak (d-s, d-l, zur)

auzoko (1): hauzocoac (arn)

azaro (1): Açarouen (ito)

azekia (3): acekiabat (arm), azeki (jats), azekia (buz)

azienda (12): aciénda (don), acienda (aint, zar, sen), acinda (anh, iba, d-g), azinda (aiz, arh, u-g, u-h), hazienda (get)

azkarki (2): azkarki (a-zo, iba)

azken (4): asquen (alt), azken (ahe, iri, mil)

azkenekotz (1): azquenecotz (hir)

aztal (2): aztaletan (osp), aztalian (zal)

ba (157): ba (arn, b-l, ur-p, bil), baçaquien (hir), bazakien (sar), bazakienak (zur), bazakiten (iri), baçén (ito), baçènn (bit), bacén (ain, hau, hen, don, don, don), Bacén (hau), Bacénn (hen), bâcebat (alt), bacen (zar, amo, anh, a-z, arh, arü, b-l, be-l, bida, bir, lek, soh, sarr, ünd, esp, g-i, get, ilh, its, l-b, lah, l-a, mak, müs, ora, urd, o-z, ort, izu, pag, s-g, d-g, d-i, sen, zal, dono, dona, u-g, ure, ur-p, ur-p, uzt, z-p, z-p), Bacen (amo, bel, soh, sarr, ünd, esp, g-i, get, ilh, lig, lah, mau, müs, ora, ort, izu, pag, s-g, d-i, sen, dono), basen (ürr, ban, l-o, luh, luk, mit), Basen (hel, ald), bassén (bun, ito), Bassèn (bit, bun), bassen (lig, l-s, irur), Bassen (o-z, irur), bazen (aiz, azka, bid, buz, ezt, gar, hel, iru, lekb, sar, bai, dom, suh, urk), Bazen (arh, ürr, a-s, azka, ezt, gar, lekb, larz, luk, mit, suh, u-h, urk, mil), bacien (leko), Bacien (anh, a-z, arü, bir, lar, osp, l-a, urd, zal, ur-p), bacirén (ain), baciren (lek, haz, urr), Baciren (zar, arb, arr, azk, b-l, bida, iru, izt, its, l-b, las, mak, hir, dona, ure, uzt), basien (l-o), Basiren (kan), Bazi 'en (ban), bazien (dom, u-h), Bazien (aiz, zur), bazi-in (aih), baziren (ahe, bas, zib, hal, 
jat, d-l, sar), Baziren (ame, bid, buz, lan, meh, mug, bai), Baçitçoun (arm), Bacitçun (gab), Bacitzoun (iba), bacitzun (iho), Bacitzun (hoz), Bazitzün (d-b), bazitzun (d-s, izp), Bazitzun (jats, lak), baziutzun (bes), baçuala (gab), baçouela (ainh), baçun (mar), Baçututçün (g-z), Bazututzun (esk), Baçutuçun (altz, laka), bazutuzun (bar), baçuçu (ath), baçouçoun (arm, iba, jut), Baçouçoun (jut), baçuçün (g-z), baçuçun (ainh, a-zo, bard, g-zo, etx, etxa, gab, ida, laka, l-z, mau, men, mend, arro, d-g, u-g, bil), Baçuçun (ainh, a-zo, beh, bard, g-zo, etxa, ida, larr, l-z, mar, men, mend, arro, ath, bil), Baçucun (alt, etx), baçuzun (hoz), Bassoussoun (ost), bassoussoune (ost), bazouzoun (gam), Bazouzoun (gam), bazuzun (alo, bes, d-b, esk, garr, izp, jats, lak), Bazuzun (alo)

bai (10): bai (a-zo, g-zo, garr), bai (hal, hal, jat, jat, urd, d-l), bay (ur-p)

baina (442): baina (aiz, arr, arh), Baina (meh), bainan (aha, ain, arb, azka, bun, eih, kan, kan, kan, hel, haz, iru, ito, ito, jats, lak, lekb, lah, lah, lar, lar, luk, meh, mug, d-g, sen, bai, dona, ure), Bainan (aha, aiz, aiz, ain, ain, ahe, anh, arb, arb, arr, arh, bun, bun, eih, kan, kan, hel, hel, haz, iru, jats, lak, lekb, lekb, lah, lar, las, luk, luk, meh, mug, mug, d-g, sen, don, don, bai, bai, ure, ure), baïnann (hen), Bainann (hen, hen), Bagnan (bida, ur-p), baiñan (zib, zib), baignan (bida, bida, bida), baina (beh, lan), bainan (ahe, ahe, ame, anh, arn, azk, aih, b-l, ban, ban, bas, bas, bid, lek, lek, lek, bes, d-s, d-b, esp, ezt, get, hal, hoz, iri, iri, iru, izp, izt, its, jat, las, ald, luh, luh, mak, mar, leko, ort, d-l, d-l, d-i, don, hir, hir, hir, hir, sar, zur, dono, dom, dom, dom, suh, u-g, urr, uzt, mil), Bainan (aha, ahe, ame, ame, anh, arr, azk, azk, azka, azka, aih, aih, arn, beho, b-l, b-l, ban, ban, bas, bid, bid, lek, bes, bes, d-s, d-b, esp, esp, ezt, ezt, get, get, hal, hoz, hoz, iho, iru, izp, izp, izt, izt, its, its, jat, lan, lan, lar, las, ald, ald, luh, mak, mak, mar, leko, leko, ort, ort, d-l, d-l, d-g, d-i, d-i, hir, hir, sar, sar, zur, zur, dono, dono, dona, dona, dom, dom, suh, suh, u-g, u-g, urr, urr, uzt, uzt, mil, mil), bana (zar, arm, bard, buz), Bana (zar, zar, arm, arm, bard, buz, buz, iho, jats, lak, urk, urk), béna (a-z, bit, hau, iba, ito, ito, l-s, ost, irur, irur, z-p), Béna (a-z, a-z, beh, beh, bit, bit, etx, etx, iba, iba, jut, l-s, l-s, ost, ost, o-z, o-z, irur, z-p), baynan (a-s), Baynan (a-s), beïnan (haz), bena (ainh, alt, alo, amo, a-zo, a-zo, arü, ürr, altz, altz, bar, bar, be-l, g-z, g-zo, soh, sarr, ünd, esk, etx, etxa, gab, gam, gar, garr, garr, garr, g-i, ida, ida, iho, ilh, jut, l-b, laka, lig, lig, larz, larr, osp, l-z, l-a, l-o, mau, men, mend, mit, müs, ora, urd, o-z, izu, pag, arro, s-g, zal, ath, u-h, urk, bil, z-p), Bena (a-m, ainh, ainh, alo, alo, amo, amo, a-zo, a-zo, arh, arü, arü, ürr, ürr, altz, bar, bard, be-l, be-l, beho, d-s, g-z, g-z, g-zo, g-zo, soh, soh, sarr, sarr, ünd, ünd, esk, esk, etxa, etxa, gab, gab, gam, gam, gar, gar, garr, g-i, g-i, hau, hau, ida, ilh, ilh, jut, l-b, l-b, laka, laka, lig, lig, larz, larz, larr, larr, osp, osp, l-z, l-z, l-a, l-a, l-o, l-o, mar, mau, mau, men, men, mend, mend, mit, mit, müs, müs, ora, ora, urd, urd, izu, izu, pag, pag, arro, arro, s-g, s-g, zal, zal, ath, ath, irur, u-h, u-h, bil, bil, $z$-p), bena (a-m. beho)

baino (147): baino (ain, ahe, ame, arb, arr, bun, eih, kan, haz, jats, lah, luk, mug, don, bai, ure), baïnonn (hen), bagno (bir, ur-p), bagnon (bir, ur-p), Bagnon (bir, bir, ur-p), baiño (zib), bainago (mil), baino (arn, azka, b-l, ban, bas, beho, bid, lek, esp, ezt, get, hal, iri, iru, izp, izt, its, jat, lar, las, luh, mak, meh, leko, ort, d-l, d-i, hir, sar, zur, dono, dona, dom, suh, u-g, urr, uzt), béino (ito), bano (aha, aint, zar, anh, arm, bard, bes, buz, iho, lak), béno (ainh, a-z, beh, etx, hau, iba, l-s, ost, o-z, ath, z-p), bayno (a-s), beino (lan), beno (a-m, aiz, alt, alo, amo, a-zo, arh, arü, ürr, altz, aih, bar, be-l, d-s, g-z, g-zo, soh, sarr, d-b, ünd, esk, etxa, gab, gam, gar, garr, g-i, hel, hoz, ida, ilh, jut, l-b, laka, lekb, 
lig, larz, larr, osp, l-z, l-a, l-o, mar, mau, men, mend, mit, müs, ora, urd, izu, pag, arro, s-g, d-g, zal, irur, u-h, urk, bil)

baizik (135): baïci (zar, pag), baïcic (ain, aint, alt, amo, anh, arü, altz, be-l, g-z, etx, etxa, hau, hen, ida, iru, lig, lah, l-z, mug, müs, o-z, arro, s-g, sen, u-h, urr), baïcik (arb, arr, arh, buz, ünd, laka, ora, don, ath), béçic (bit, bit), baïsic (kan, l-s, luk), baïssic (bun, irur), baïzik (aiz, alo, gar), baicic (ainh, arn, azk, bid, soh, g-i, hoz, iri, izt, its, las, osp, l-a, mak, mar, mau, urd, hir, dona, dom, u-g, ure, uzt, z-p), béicic (ito), baicik (sarr, esp, leko, men, d-g, zal), baiciq (eih, larr), baisic (ürr, ald), baisik (l-o, luh, mit), baizic (bas, esk, ezt, hal, izp, jat, dono, suh), baizik (ahe, azka, bar, jats, lekb, zur, mil), baycic (bil), bécic (aha, a-z, iba), bécik (beh, ost), becic (a-zo, arm, beho, gab, jut, lan, izu), becik (a-m, ilh, l-b, mend), beïcic (meh), beïzic (hel), beicic ( $\mathrm{g}$-zo), beicik (bard, bida), beizic (iho, lak), besic (ban), beyzik (a-s), bezic (aih, bes, larz, bai), bezik (d-s, d-b, gam, garr), bezin (urk)

bakar (7): bakar (bida), bakarbat (esp), bakhar (iru, don), bakharbatçuetan (hir), bakharra (its), bakharrak (zib)

balle (6): baille (beho, izp), baillea (suh), balea (b-l), Balea (las), vaillea (d-i)

bana (3): bana (aint), Bana (aint)

baño (1): bagnore (jut)

baratu (50): baatou (bun), baatu (a-z, arü, g-zo, sarr, d-b, gab, ilh, mend, ost, izu, pag), baâtu (a-zo), baraazten (d-b), baratcen (etxa, arro, zal, bil), baratou (a-s, buz, gam, jut, lekb, dona), baratséntu (ito), baratu (amo, arn, beho, d-s, ünd, etxa, gar, hal, izp, jat, jats, laka, lan, larz, lar, mar, men, d-l, zal, u-g, bil), baratzen (ame, ürr, esk)

baratze (144): baatcé (altz, beh, iba, ida, ito, ost), baatce (bir, gab, laka, l-z, izu, pag, ath, ur-p), baatceat (g-zo, mend), baatciat (a-zo), baatsé (g-z, lig, l-s, irur), baatse (o-z), baatsia (urd), baatze (a-m, aha, d-b, gam, hel), baâtze (zur), baatzet (urk), baatziakin (bas), baàtze (azka), bahatze (haz), baratcé bat (ain, aint, zar, a-z, bit, hen, izt, lah, don, dona), baratcébat éta (bun), baratce bat (aiz, ainh, amo, anh, arb, arn, beho, eih, arr, azk, b-l, be-l, bid, soh, sarr, ünd, esp, etx, etxa, g-i, get, hoz, its, jut, lan, larr, las, ald, osp, mak, mau, meh, leko, men, ort, s-g, sen, zal, suh, ure, urr, uzt), baratce eta (bida, luh, arro), baratce tchipi (müs), baratce (bil), baratcearequin (hir), baratcebat eta (arü, lar, mug), baratcebat éta (z-p), baratsé (kan), baratze bat (ahe, arh, ürr, aih, ban, bar, lek, bes, d-s, buz), baratze chume (zib), baratze bat (esk, ezt, gar, hal, iho, iri, iru, izp, jat, jats, lak, lekb, larz, l-a, luk, mit, d-i, sar, dono, bai, dom, u-h), baratzearekin (d-l), baratzeatekin (garr), baratzebat eta (alo, a-s, l-o, ora, mil), baratziarequin (ame), batcé (hau), batce (arm, ilh), batceat (bard, l-b)

baratzeño (3): baratce gnobat (mar, d-g), baratceñobat ( $u-g$ )

Barbau (4): Barbaou (o-z, o-z, o-z, o-z)

Barbazan (224): Babazanéco (a-z), Barbanazeco (l-s), Barbanzaco (d-l), Barbanzako (d-l), Barbanzaneco (lig), Barbasan (luk), Barbasaneco (etx, etx, l-z, l-z), Barbasanéco (kan), Barbasango (hen, zur, zur), Barbassaneco (l-b), Barbassaneko (ilh, ilh), Barbasseneko (l-b), Barbazan (ain, ame, anh, azk, aih, beh, bas, bid, lek, kan, g-z, d-b, esp, gam, garr, haz, ida, lekb, lan, ald, luk, müs, o-z, don, sar, bil), BARBAZAN (arr, bas), Barbazan (beho), Barbazanco (eih, get, hal, jat, u-h, uzt), Barbazanéco (ain, a-z, bit, bun, gab, lah, l-s, ost, ath), Barbazaneco (aiz, zar, ainh, amo, a-zo, arh, arh, arm, a-s, altz, altz, b-l, b-l, ban, bard, be-l, be-l, bit, bir, lek, bes, bes, d-s, d-s, g-zo, soh, soh, sarr, ezt, 
ezt, etxa, g-i, g-i, get, hel, hau, hoz, hoz, iba, iri, iri, iru, iru, izp, izt, izt, jut, lak, laka, lig, larz, larr, lar, las, ald, osp, l-a, l-o, mak, mar, mau, meh, leko, mend, mit, mit, mug, ora, urd, urd, urd, ort, ort, izu, pag, pag, arro, d-g, d-i, hir, zal, zal, dono, bai, bai, dona, dona, dom, suh, suh, irur, irur, u-g, u-g, u-h, urk, ure, ure, ur-p, z-p, a-m, aha, aint, arn, beho), Barbazan-eco (esk), Barbazanecoua (luh), Barbazaneko (alo, arü, ürr, azka, bard, buz, ünd, gar, laka, l-o, leko, men, men, mend, d-g, sar, mil, bil), Barbazane-ko (jats), Barbazanen (alt, alt, ame, arm, bid, buz, iba, osp, müs, ost), Barbazan-en (bar), Barbazanen (jut), Barbazaneren (luh), Barbazango (arb, arr, bida, zib, esp, hal, its, jat, sen, urr), Barbazan-go (zib), Barbazen (bun), Barbazeneco (anh, iho, larz, l-a), Barbazen-en (its), Barbazzen-eko (bar), Barbezan (ahe), Barbezanéco (ito), Barbezaneco (s-g), Barbézanén (ito)

barku (1): barkhuric (arn)

barna (4): barna (a-zo, g-zo, lar, hir)

barne (36): barnéat (bit), barnérat (lah), barne'at (ban, ban), barnean (zib, d-l), barneat (altz, altz, garr, iho, urr), barnen (l-o, mar), barnera (ame, lekb), barnerat (arb, arr, b-l, bid, bes, zib, hoz, hoz, iho, iru, ald, luh, luh, mug, arro, hir, urr, uzt, z-p), barnetan (lan), barnez (d-l)

basa (67): bacha (aha, ito, dom), basa (alo, arb, arn, arr, arm, aih, ban, bir, d-s, g-z, d-b, esp, esk, ezt, etx, gar, hel, hal, haz, hoz, iri, izp, izt, jat, laka, lekb, larz, las, l-z, l-a, luk, leko, mug, ort, suh, urk, uzt), basascoa (sar), basauzu (iho), bassa (ain, aint, zar, ainh, a-z, azka, bun, ilh, l-b, lig, larr, lar, osp, l-o, müs, urd, o-z, arro, s-g, zal, irur, ure), bassaou (beh), baza (ame, garr)

basatiar (1): basatiara (zib)

bat (2678): at (izu, izu), atsoat (bard), baatceat (mend, g-zo), baatzet (urk), baatciat (a-zo), batés (bit), bat (ald), batez (a-m, aha, aint, arn, beho, eih, arm, a-s, beh, bard), beceatetan (bard), behignoat (a-zo, g-zo), berceat (leko), bertzeatian (bas), besteat (d-s, g-zo), bestéat (ito), besteatian (gab), bezeatetan (urk), bezteat (garr), borthaat (leko, g-zo), bortaatetan (urk), borthaatian (gab, hel), borteatian (aiz), chipignoat (g-zo), elibat (müs, g-i, laka), élibat (alt), elibat (arro, zal, irur), élibat (altz), élibatec (altz), elibatec (urd, esk), elibatetan (be-l, soh, osp, bil, urd), elibatez (mit, ürr), élibatez (altz), elibatez (bar, be-l, soh, esk, g-i, bil, bil, bar, ida, osp), emazteat (bard, g-zo), emaztet (urk), etcheat (garr, mend), etcholat (osp), guiçounbat (a-zo), gninignoatek (bard), gnobat (mend, d-g, mar), molchoat (aiz), parteat (bir), saldoat (jats), tropaat (mend, beh, bes, g-zo), tropa'at (ban), tropat (urk, bard, bir, garr, ilh, l-b), ttipiñoatek (d-b), çabalbat (esp, urd), çaharbat (lek, lar, l-s, mar, mug, hir), acekiabat (arm), ñimiñobat (u-g), aldebat (lar), aldebatez (l-o), aldibates (lar, mar), émastebat (etx), émaztékibat (lah), çouinbat (alt), arastiribatez (ida), arditropabat (mar), arguibates (alt), aristiibates (irur), aristiribatez (pag, z-p), ariztiibatez (lig), arratsaldebates (esp), arratsaldebatez (hir), arrocabat (bun), arrocabaten ( $z-p)$, artebatetako (a-s), arthaldébat (kan), arthaldebat (hir), 'ten (ilh), 'tian (ilh), baat (ito), béhibat (z-p), bakarbat (esp), baratcébat (bun), baratceñobat (u-g), baratcebat (arü, mug, z-p), baratzebat (a-s, ora, mil), bértcébati (don), basterbatian (esp), bat (a-m, aha, aint, arn, beho, eih, aiz, aiz, aiz, aiz, aiz, aiz, aiz, ain, ain, ain, ain, ain, ain, ain, ain, ain, zar, zar, zar, zar, zar, zar, zar, zar, zar, zar, ahe, ahe, ahe, ahe, ahe, ahe, ahe, ainh, ainh, ainh, ainh, ainh, ainh, ainh, alt, alo, ame, ame, ame, ame, ame, ame, ame, ame, , amo, amo, amo, amo, amo, 
amo, amo, amo, anh, anh, anh, anh, anh, anh, anh, anh, a-z, a-z, a-z, a-z, a-z, a-z, a-z, a-z, arb, arb, arb, arb, arb, arb, arb, a-zo, a-zo, arr, arr, arr, arr, arr, arr, arr, arr, arr, arh, arh, arh, arh, arh, arh, arh, arh, arm, arm, arm, arm, arm, arm, arm, arü, arü, ürr, ürr, ürr, ürr, ürr, ürr, ürr, ürr, ürr, ürr, a-s, a-s, a-s, a-s, azk, azk, azk, azk, azk, azk, azk, azk, azk, azk, azka, azka, azka, azka, azka, azka, azka, azka, altz, altz, altz, altz, altz, altz, aih, aih, aih, aih, aih, aih, aih, aih, aih, aih, beh, beh, beh, beh, beh, beh, beh, b-l, b-l, b-l, b-l, b-l, b-l, b-l, ban, ban, ban, ban, ban, ban, ban, ban, bar, bar, bar, bar, bar, bar, bar, bar, bar, bar, bar, bard, bard, bard, bard, bas, bas, bas, bas, bas, be-1, be-l, be-l, be-l, be-l, be-l, be-l, be-l, bit, bit, bit, bit, bit, bit, bit, bit, bit, bid, bid, bid, bid, bid, bid, bida, bida, bida, bida, bida, bida, bida, bir, bir, bir, bir, bir, bir, bir, lek, lek, lek, lek, lek, lek, lek, lek, lek, lek, lek, lek, bes, bes, bes, bes, bes, bes, bes, bes, bun, bun, bun, bun, bun, bun, bun, d-s, d-s, d-s, d-s, d-s, d-s, d-s, d-s, d-s, d-s, d-s, buz, buz, buz, buz, buz, buz, buz, buz, kan, kan, kan, kan, kan, kan, g-z, g-z, g-z, g-z, g-z, g-z, g-z, g-z, g-zo, g-zo, g-zo, g-zo, soh, soh, soh, soh, soh, soh, soh, soh, sarr, sarr, sarr, sarr, sarr, sarr, sarr, sarr, sarr, zib, zib, zib, zib, zib, zib, zib, zib, d-b, d-b, d-b, d-b, d-b, d-b, d-b, d-b, ünd, ünd, ünd, ünd, ünd, ünd, ünd, ünd, ünd, esp, esp, esp, esp, esk, esk, esk, esk, esk, esk, esk, esk, ezt, ezt, ezt, ezt, ezt, ezt, ezt, ezt, etx, etx, etx, etx, etx, etx, etx, etx, etx, etxa, etxa, etxa, etxa, etxa, etxa, etxa, gab, gab, gab, gab, gab, gam, gam, gam, gam, gam, gar, gar, gar, gar, garr, g-i, g-i, g-i, g-i, g-i, get, get, get, get, get, hel, hel, hel, hel, hal, hal, hal, hal, hal, hal, haz, haz, haz, haz, haz, haz, haz, hau, hau, hau, hau, hau, hau, hau, hau, hen, hen, hen, hen, hen, hoz, hoz, hoz, hoz, hoz, iba, iba, iba, iba, iba, ida, ida, ida, ida, ida, ida, iho, iho, iho, iho, iho, iho, ilh, ilh, ilh, iri, iri, iri, iri, iri, iri, iri, iri, iru, iru, iru, iru, iru, izp, izp, izp, izp, izp, izp, izp, izp, izt, izt, izt, izt, izt, izt, izt, izt, ito, ito, ito, ito, ito, its, its, its, its, its, its, jat, jat, jat, jat, jat, jat, jats, jats, jats, jats, jut, jut, jut, jut, jut, l-b, l-b, l-b, lak, lak, lak, lak, lak, lak, lak, laka, laka, laka, laka, laka, laka, laka, lekb, lekb, lekb, lekb, lekb, lig, lig, lig, lig, lig, lig, lah, lah, lah, lah, lah, lan, lan, lan, lan, lan, lan, larz, larz, larz, larz, larz, larz, larr, larr, larr, larr, larr, larr, larr, larr, larr, lar, l-s, l-s, l-s, l-s, las, las, las, las, las, ald, ald, ald, ald, ald, ald, osp, osp, osp, osp, osp, l-z, l-z, l-z, l-z, l-z, l-z, l-z, l-z, l-a, l-a, l-a, l-a, l-a, 1-o, l-o, l-o, luh, luh, luh, luh, luh, luh, luh, luk, luk, luk, luk, luk, mak, mak, mak, mak, mak, mak, mau, mau, mau, mau, mau, mau, mau, mau, mau, mau, meh, meh, meh, meh, meh, meh, meh, meh, meh, leko, leko, leko, leko, leko, leko, leko, leko, men, men, men, men, men, men, men, men, mend, mend, mend, mit, mit, mit, mit, mit, mit, mug, mug, mug, mug, müs, müs, müs, müs, müs, müs, müs, müs, ora, ora, ora, ora, ora, urd, urd, urd, urd, urd, ost, ost, ost, ost, ost, ost, ost, ost, ost, ost, o-z, o-z, o-z, o-z, $\mathrm{o}-\mathrm{z}, \mathrm{o}-\mathrm{z}, \mathrm{o}-\mathrm{z}, \mathrm{o}-\mathrm{z}$, ort, ort, ort, ort, ort, ort, ort, ort, izu, izu, izu, izu, izu, izu, pag, pag, pag, pag, pag, pag, pag, arro, arro, arro, arro, arro, arro, s-g, s-g, s-g, s-g, s-g, s-g, s-g, s-g, d-l, d-l, d-l, d-l, d-l, d-l, d-l, d-l, d-g, d-g, d-g, d-i, d-i, d-i, d-i, d-i, d-i, d-i, d-i, sen, sen, sen, sen, sen, sen, sen, don, don, don, don, don, don, don, don, don, don, hir, hir, hir, hir, hir, hir, hir, sar, sar, sar, sar, sar, sar, sar, sar, sar, zal, zal, zal, zal, zal, zal, zal, zal, zal, zal, zur, zur, zur, zur, zur, zur, zur, dono, dono, dono, dono, dono, dono, dono, dono, bai, bai, bai, bai, bai, bai, bai, bai, dona, dona, dona, dona, dona, dona, dona, dona, dona, dona, dom, dom, dom, dom, dom, dom, dom, dom, dom, suh, suh, suh, suh, suh, suh, suh, suh, suh, suh, ath, ath, ath, ath, ath, ath, ath, ath, ath, ath, irur, irur, irur, irur, irur, irur, irur, u-g, u-g, u-g, u-h, u-h, u-h, u-h, u-h, u-h, u-h, u-h, urk, 
urk, urk, urk, urk, ure, ure, ure, ure, ure, ure, ure, ure, ur-p, ur-p, ur-p, ur-p, ur-p, ur-p, ur-p, ur-p, ur-p, urr, urr, urr, urr, urr, urr, urr, urr, urr, uzt, uzt, uzt, uzt, uzt, uzt, uzt, uzt, uzt, uzt, mil, mil, mil, mil, bil, bil, bil, bil, bil, bil, z-p, z-p, z-p, z-p), bat) (ban, hel), Bat (jut, dona), baté (a-z, g-z, g-z, hau, ilh, ito, l-s, ath), batê (bai, bai), batéé (beh, etx), Batéé (alt), batéan (ain, ain, ain, ain, lah, lah, lah), batéänn (hen), bat'étan (ath), batèc (bit, hen, hen, ito), batéc (ain, ain, ain, kan, kan, l-s, l-s, ost, ost), batée (gam, gam), baték (beh, don, don), batén (ain, ain, l-s, ost, ost, don), baténn (hen, hen), batès (bun, ito), batés (alt, bit, kan, kan, hau, hau, ito, l-s, l-s, ost, ost), batétako (don), batétan (etx, hau, hau, ito, ito, ito, ito, mug), batéz (ain, ain, ain, bit, hen, iba, don), bate (alo, arm, bard, bir, iba, ilh, l-b, l-b, l-o, men, men, mend, o-z, izu, ath), bate'e (ban), batean (aiz, ahe, ahe, ahe, ame, arm, arn, a-s, azk, azk, b-l, b-l, b-l, b-l, bid, bida, bida, lek, zib, zib, get, get, hal, haz, iri, iri, iru, iru, iru, izt, izt, izt, its, its, its, its, l-b, lar, ald, ald, luh, luh, mak, meh, leko, leko, ort, ort, ort, ort, d-l, d-l, d-l, d-l, d-i, d-i, sen, sen, hir, hir, sar, sar, sar, ure, ure, ure, ure, ure, uzt, uzt, mil, mil, mil, mil, mil), bateat (ur-p), batec (a-m, aha, aint, arn, beho, eih, zar, zar, zar, ahe, ahe, ainh, ainh, ainh, ainh, alt, ame, ame, amo, amo, anh, anh, a-z, a-z, a-z, arb, a-zo, a-zo, arr, arh, arh, arh, arm, arm, arm, arü, azk, azk, azk, azka, altz, aih, aih, b-l, ban, ban, ban, bas, bas, be-l, be-l, be-l, bid, bid, bid, bir, bir, bir, bes, bes, bes, bun, bun, bun, d-s, buz, g-z, g-z, g-zo, g-zo, soh, soh, soh, esk, esk, ezt, etx, etxa, etxa, etxa, gab, gab, gab, g-i, g-i, g-i, get, get, get, hel, hel, hal, haz, haz, hau, hau, hau, hen, hoz, hoz, hoz, iba, iba, ida, ida, iho, iho, iri, iri, iru, iru, iru, izp, izp, izp, izt, izt, ito, its, its, jat, jats, jut, jut, jut, lak, lak, lak, laka, laka, laka, lekb, lig, lig, lah, lah, lah, lan, lan, lan, larz, larz, larr, larr, lar, las, las, ald, ald, osp, l-z, l-z, l-a, l-a, luk, luk, mak, mak, mak, mar, mau, mau, mau, meh, meh, leko, mend, mend, mit, mit, mug, müs, müs, müs, ora, ora, ora, urd, urd, o-z, o-z, o-z, ort, ort, ort, izu, izu, izu, arro, arro, arro, s-g, s-g, s-g, d-i, d-i, d-i, sen, sen, sen, sen, hir, hir, hir, zal, zal, dono, dono, bai, bai, bai, dona, dona, dona, dom, dom, suh, suh, suh, ath, ath, ath, irur, irur, u-h, u-h, u-h, ure, ure, ure, ur-p, urp, ur-p, urr, urr, urr, uzt, uzt), bateco (lak), batee (bes, hel, l-z, leko, leko), batek (aiz, aiz, aiz, ahe, alo, alo, arb, arr, ürr, ürr, ürr, a-s, a-s, altz, beh, beh, bar, bar, bard, bida, bida, lek, d-s, d-s, buz, sarr, sarr, zib, zib, zib, d-b, ünd, esp, esp, ezt, etx, gam, gam, gam, gar, gar, garr, garr, garr, haz, ilh, jats, jats, l-b, lekb, lig, las, l-o, luh, luh, leko, leko, men, men, men, urd, d-l, d-l, sar, zur, zur, u-g, urk, mil, mil, mil, bil, bil), batekin (lek, sar), baten (a-m, aint, arn, beho, eih, zar, ahe, ainh, ame, ame, ame, amo, anh, a-z, arb, arb, a-zo, arr, arr, arm, arm, ürr, ürr, a-s, a-s, azk, azka, aih, beh, beh, b-l, ban, bar, bar, bard, bas, bid, bida, bir, lek, bes, bes, d-s, buz, g-zo, zib, zib, d-b, d-b, ünd, esp, ezt, etx, etxa, gab, gam, gam, g-i, get, hel, hel, hal, hal, haz, hoz, iba, ida, iho, iho, ilh, iri, iru, iru, izp, izp, izt, its, its, jat, jat, jats, jats, jut, jut, l-b, lekb, lah, lan, lan, larz, lar, las, las, ald, osp, l-z, l-a, luh, luk, mak, mak, mar, mau, meh, meh, leko, leko, men, men, mit, mit, müs, ora, ora, o-z, ort, ort, pag, s-g, d-l, d-l, d-g, sen, sen, hir, sar, sar, zur, dono, dono, bai, bai, dona, dona, suh, ath, ath, u-g, urk, ure, ur-p, urr, urr, uzt, uzt, mil, mil), batenn (bit), batequi (be-l, soh), batequin (hir), bates (aha, eih, alt, arh, ban, ban, bun, bun, g-z, g-z, g-z, esp, lig, luh, luh, mau, men, o-z, o-z, dono, ath), batetaco (b-l, bas, las), batetan (ainh, ainh, ainh, alt, alo, , amo, amo, amo, amo, amo, a-zo, a-zo, a-zo, a-zo, arü, arü, ürr, ürr, ürr, altz, altz, bar, bar, bard, bard, be-l, be-l, be-l, be-l, be-l, be-l, bes, buz, buz, g-z, g-z, g-z, g-z, g-zo, soh, soh, soh, soh, soh, soh, 
sarr, sarr, sarr, ünd, ünd, esk, esk, esk, esk, etx, etxa, etxa, etxa, etxa, gar, gar, garr, g-i, g-i, g-i, hau, hau, ida, ida, ida, laka, laka, laka, laka, lekb, lekb, lig, lig, lig, lig, larr, larr, larr, larr, osp, osp, osp, osp, osp, l-z, l-z, l-a, l-a, mau, mau, mau, men, men, men, men, mit, mit, mit, mit, mug, müs, müs, müs, urd, o-z, o-z, o-z, o-z, arro, arro, arro, arro, arro, arro, s-g, s-g, s-g, s-g, s-g, zal, zal, zal, zal, irur, irur, irur, bil, bil, bil, bil), batetara (ürr), Batetic (lar), batex (azk), batez (aiz, aiz, zar, zar, ahe, ahe, ahe, ahe, ainh, ainh, alo, alo, ame, amo, amo, anh, anh, a-z, a-z, arb, arb, a-zo, a-zo, arr, arr, arh, arm, arm, arü, arü, ürr, ürr, a-s, a-s, azk, azka, azka, altz, altz, aih, aih, aih, beh, b-l, b-l, bar, bar, bard, bas, bas, be-l, be-l, bid, bid, bida, bida, bir, lek, lek, bes, bes, d-s, d-s, buz, buz, g-z, g-zo, g-zo, soh, soh, sarr, sarr, zib, zib, zib, d-b, d-b, ünd, ünd, esk, esk, ezt, ezt, etx, etxa, etxa, gab, gab, gam, gam, gar, gar, gar, garr, garr, garr, g-i, g-i, g-i, get, get, hel, hel, hal, hal, haz, haz, hen, hoz, hoz, iba, ida, iho, iho, iho, ilh, ilh, iri, iri, iri, iru, iru, izp, izp, izt, izt, its, its, jat, jat, jats, jats, jut, jut, l-b, l-b, lak, lak, laka, laka, lekb, lekb, lah, lah, lan, lan, larz, larz, larr, larr, las, las, ald, ald, osp, osp, l-z, l-z, l-a, l-a, l-o, luk, luk, mak, mak, mar, mau, mau, meh, meh, leko, leko, mend, mit, mit, mug, mug, müs, müs, müs, ora, ora, urd, urd, ort, ort, izu, izu, arro, arro, s-g, s-g, d-l, d-g, d-g, d-i, sen, sen, don, hir, sar, sar, zal, zal, zur, dono, bai, bai, dona, dona, dom, dom, suh, suh, ath, irur, u-g, u-g, u-h, u-h, urk, ure, ure, ur-p, ur-p, urr, uzt, uzt, mil, bil, bil, z-p), batezn (etx), bathéé (beh), bathez (men, zur), bathi (ald), bati (aiz, ahe, a-z, a-z, arm, arm, azka, azka, bir, bir, ald, d-i, d-i, sen, sen, don), batian (a-m, aha, aint, beho, eih, zar, zar, ame, ame, ame, anh, anh, anh, a-z, a-z, a-z, arb, arb, a-zo, arr, arr, arh, arh, arh, arh, arh, arm, a-s, a-s, azk, azka, azka, azka, aih, aih, aih, beh, beh, beh, beh, ban, ban, ban, bas, bas, bas, bid, bid, bid, bid, bir, bir, bir, bir, lek, lek, bun, bun, d-s, d-s, d-s, buz, buz, buz, kan, kan, kan, kan, kan, kan, g-zo, g-zo, d-b, d-b, d-b, d-b, esp, esp, esp, ezt, ezt, ezt, ezt, ezt, ezt, gab, gab, gam, gam, gam, gam, get, get, hel, hel, hal, hal, hal, hal, haz, hoz, hoz, hoz, hoz, hoz, iba, iba, iba, iba, iba, iho, iho, ilh, ilh, iri, iru, izp, izp, jat, jat, jat, jat, jat, jats, jats, jats, jats, jut, jut, jut, jut, l-b, l-b, l-b, lak, lak, lak, lekb, lekb, lekb, lah, lah, lan, lan, lan, lan, larz, larz, larz, larz, larz, lar, l-s, l-s, l-s, l-s, las, las, las, ald, l-a, l-o, l-o, l-o, l-o, luk, luk, luk, luk, luk, mak, mak, mar, mar, meh, mend, mend, mend, ora, ora, ora, ora, ost, ost, izu, izu, izu, izu, izu, d-g, d-g, d-i, d-i, don, don, zur, zur, zur, zur, zur, dono, dono, dono, dono, bai, bai, bai, bai, dona, dona, dona, dom, dom, dom, dom, dom, dom, suh, suh, suh, u-g, u-g, u-g, u-g, u-h, u-h, u-h, u-h, u-h, ur-p, ur-p, urr, urr, z-p, z-p), batiane (ost), batiann (bit, bit, bit, hen, hen, hen), batin (pag), batt (iru), batzen (aih, larz), behiñobat (u-g), behibat (arü, a-s, esp), behignobat (d-g), bertcebat (mug), bertcebatian (esp), bestébat (bun, irur), bestélibatetan (o-z), bestebat (alo, arü, esk, g-i, l-a, pag, z-p), bestebatetan (ida, bil), bestebati (gar), bestebatian (mar, mar), bestelibati (gar), beztebat (lig), bihibat (bard, d-b, get), bortabat (arü), borthabatetan (bard), borthabati (gar), bortubatetan (gar), botchebaten (1-o), chaharbat (alt, urd), chipibat (arü, mit, z-p), chipibatec (pag, pag, z-p, z-p), chipibatetan (altz), chortabat (lar), coimbat (mar), coumbat (etx), eguerdi (mend), Egumbates (lar), Egunbatez (pag), ejerbatétan (alt), ejerbatetan (alo, urd), ekhuratubat (g-i), emastebat (arü, ilh, mar, pag), emastekibat (esp, mug), emaztebat (aiz, alo, l-b, urd, mil), estainubat (arb), etchebat (larr), gnimignobat (mar, ora, d-g), gnimignobatek (ilh, ilh, l-b, l-b), gueldibat (a-s, mar), guiçonbat (mar, müs), handibat (aiz, alt, alo, arü, kan, esk, müs, hir), handibatetan (mug), harphegnabaten (pag), 
haudibat (lar), haundibat (zur), heribatetan (gar, bil), heribatian (l-o, mar), heriignobatian (d-g), herribatean (lar), herribatetan (arü, bes, urd), herribatian (pag), hilabateko (mil), Ilhuntcebatez (lar), Ilhuntzebatez (hir), inkarubat (ida), karoumbat (mar), kharouinbat (l-o), kharrouïnbat (garr), kharroubat (z-p), léisebat (l-s), Laboaïbatec (irur), laboraribatec (arü, b-l, mit, z-p), Laboraribatec (mar, mug, pag), laboraribatek (ünd, u-g, urk), Laboraribatek (a-s), lacubat (urr), leicebat (d-g, u-g), leihobat (mar), lekhubatetan (alt), loskobat (ath), mendibatean (lar, hir), mendibaten (anh, esp), mendibatetan (altz, mug), mendibatian (d-g, u-g, z-p), mountagnabateen (ida), nechkabatec (lar), oyhanbatétara (bit), paropiabatétan (irur), patian (ur-p), pollitbatian (anh), poulibatian (d-g), poutsoubat (bun), saharbat (bun, l-o, z-p), saldobat (alo, arü, gar, l-s, l-o, mit, pag), (silobat (z-p), soumbat (ito, l-s, irur), tcébatez (alt), tchipibat (ida), tchipibatek (d-g, d-g), thipibatec (alt), tipibatek (l-o), tropabat (alt, a-zo, b-l, mil, z-p), ttipibat (a-s, esk, arro, mil), ttipibatec (amo, azka, azka), ttipibatek (u-g), ttipittobat (mug), zaharbat (alo, a-s, ünd, urk)

batere (143): bate (a-m), etcheate (izu), bat_èré (bun), bat_éré (zar, lig, irur), batéré (a-z, bit, hen, ito, ost, z-p, z-p), Batéré (z-p), batére (ainh, mau), batere (arn, beho, aiz, aiz, ahe, ahe, amo, amo, anh, a-zo, arü, arü, ürr, ürr, a-s, a-s, azk, azka, b-l, b-l, ban, be-l, be-l, buz, buz, g-zo, soh, soh, sarr, sarr, zib, ünd, ünd, esk, ezt, etxa, etxa, gab, gab, gar, gar, g-i, g-i, haz, ida, ida, iho, izt, jat, jats, jats, lak, lak, laka, laka, lekb, lekb, lan, lan, larz, larr, las, las, osp, l-a, l-a, l-o, luh, luk, mak, mak, mar, mau, mit, mit, mug, müs, müs, ora, urd, ort, ort, pag, pag, arro, arro, s-g, d-i, hir, sar, zal, suh, u-g, ure, urr, urr, uzt, mil, bil, bil), bat_ere (arh, bes, hal, hoz, izp, u-h), Batere (b-l, buz, etxa, gab, hoz, ida, jut, laka, müs, d-g, u-g, bil), batere_gabe (osp), bateren (mar), Bathere (zib), batre (anh, bid, bid, ure), Batre (anh, eih)

batu (2): batceco (urd), bâtu (1-b)

batzuk (165): arrokatzutan (garr), besteatcietan (gab), besteatzuetan (hel), besteatçutan (mend), 'atçutan (ilh), çoratubatçuez (mug), azutan (larz), bakharbatçuetan (hir), basuec (ur-p), batçou (bit, dona), batçouétan (ain), batçouetan (eih, ora, ora), batçouez (ora), batçous (bit), batçoutan (aint, zar, iba, jut), batçu (azk, lar, leko, hir, suh), batçuc (a-zo, g-zo), batçuec (a-z, lek, hir), batçuei (d-i), batçuek (esp), batçuer (a-z), batçues (esp), batçuetan (b-l, eih, esp, esp, izt, las, ort, ure, ure), batçuez (a-z, b-l, hoz, its, its, ort, suh, suh), batçutan (a-m, amo, anh, a-zo, g-zo, l-b, ost, izu), batçuz (arü, l-b, izu, izu), batcouer (arm), batcuez (bida), batcutan (anh), batcuz (anh), batsou (bun), batsouétan (kan), batsouetan (ald), batsous (d-g), batsoutan (bun), batsoutane (ost), batsuétan (ito), batsus (u-g), batsutan (l-s), batsuz (bard), batzütan (d-b), batzi-ic (aih), batzi-itan (aih), batzouétan (lah), batzouez (buz, lah), batzouitan (buz), batzoutan (arh, gam), batzouz (gam, iba), batzu (arb, arb, arn, arr, bas, bes, zib, garr, iho, iri, iri, lak, meh), batzuétan (lah), batzuc (haz), batzuec (arb, hoz, lar), batzuek (zib, d-l), batzuer (azka), batzueri (arr), batzuetan (ezt, get, hal, iru, jat, luk, luk, d-l, d-l, sar, dono, dom, uzt, uzt, mil, mil), batzuez (aiz, ame, sarr, zib, ünd, haz, haz, iru, meh, dom, dom), batzuitan (hal, jat, bai), batzus (l-o), batzutan (bid, gam, jats, jats, lekb, lan, l-o, u-h), batzuyez (bes, bes), batzuz (beho, d-s, jats, mil), bazuec (bir), bazueri (bir), bazuetan (ur-p), bertcebatçûtan (mug), gaichtobatzuz (azka, d-s), herribatçouetan (arm)

Baxenafar (4): Bachenabarreco (ezt, dom, dom), Bachenabarreko (ezt)

baxepireneo (1): bassepireneco (mar) 
bazkalondo (2): bascal ondo (get, hen)

bazkatu (5): bascatcen (altz), baskatzen (izu), bazkatzen (aiz, gam, d-l)

bazter (132): bastérian (kan), bastériann (hau), bastérréat (ito), baster (l-s, ure), Baster (arü, ünd, men), bastera (mar), basterïn (g-z), basterbatian (esp), basteriala (lig), basterian (arh, azk, ban, hoz, ida, lekb, larr, mak, d-i, z-p), basterila (mau, urd, o-z), basterin (altz), basterrean (b-l, get, izt, leko, ure, uzt), basterretaco (ame), basterrian (ainh, ame, a-zo, a-s, be-l, bid, eih, g-zo, hel, jat, mak, mend, izu, dono, u-h, u-h), basterriànn (hen), basterrila (etxa), basterrin (pag), baztéréan (ain), baztéreean (mug), baztérian (etx, dom), baztérréra (don), baztérrian (beh), bazter (ürr, iho, meh, mit, sar, bil), Bazter (sarr), Bazteretchar (zib), bazteriala (osp, irur), bazterian (alo, a-m, anh, gar, lak, lekb, l-z, l-a, müs), bazterila (bil), bazterin (ürr), bazterrean (arn, haz, iru, ort, mil), bazterrera (suh), bazterriala (zal), bazterrian (aint, arn, zar, arh, aih, buz, soh, ezt, gab, g-i, iho, jats, jut, lan, larz, las, luk, ora, s-g, d-g, bai, dona), bazterrila (bar), bazterrin (bes, bes, esk, laka, mit, arro), pastarrian (ito), pasterean (zib), pasterrean (its), pasterrian (arb, arr, beh, lah, izu), pazterian (anh), pazterrian (ora), phasterrean (d-i), phasterrian (eih, lar), phazterin (urk), phazterrean (iri), phazterrian (lar), pphazterrin (arro)

bazter-herri (1): baster-herri (sar)

bedera (1): bedera (aiz)

begi (3): Begia (zib), beguietaric (lar, hir)

begirale (6): béguiraléic (bit), beguirale (hal, jat, luk, dona), beguiraleric (b-l)

begirari (20): bégirariik (ünd), béguiaï (g-z), béguiaic (l-s), béguiaiic (g-z), begiariric (ürr), begirari (bar), begirariik (sarr), beguiaiic (osp), beguiraik (altz), beguirari (urd, s-g), beguirariaric (etxa), beguiraric (ida, suh), beguirariic (izu, arro), beguirariric (arü, laka, müs), beguiraririk (mit)

begiratu (108): begiatcen (men), Begiatçen (men), Begiatcen (sarr, ünd), Bégiatséntu (ito), Begiatzen (ürr), Bégiatzentu (mit), begiratcen (arn, u-g), begiratcentu (d-g), Begiratzen (bar, buz, esk, l-o), béguiacen (alt), Béguiacen (alt), beguiadi (hau), béguiatcen (alt, ath), Beguiatcen (arü, be-l, soh, g-i, ida, laka, osp, ath), Béguiatcën (g-z), Béguiàtcén (hau), Béguiatcen (l-z), beguiatcen (urd, urd), Beguiatcentci (izu), Béguiatcentiçu (etx), Beguiatcentu (pag), beguiatcia (altz), Beguiatsen (irur), Béguiatsen (lig, o-z), béguiatsen (lig, o-z, irur), Béguiatsentu (l-s), beguiatzen (alo), Beguiatzen (gar), Béguiatzen (1a), beguirann (hal), béguiratcéko (don), beguiratcen (ainh, ainh, b-l, g-i, larr, mau, müs), Beguiratcen (etxa, larr, las, mar, arro, s-g, zal, dona, suh, bil), Béguiratcen (mau, don), béguiratçènn (bit), Beguiratcentu (müs), béguiratcentu (z-p), Béguiratcentu (zp), Béguiratsen (bun), Beguiratzeintu (hal, jat), Beguiratzen (ame, iru), beguiratzen (iri), Beguirauc (hal, jat), beguiraun (jat), Beira (arn), beiratcen (a-zo, izt), Beiratcen (a-m, izt, jut, mend, uzt), Béiratcén (ost), Beïratcen (aha), Beïratcentci (iba), Béiratsènn (bit), beiratzen (ahe, jats), Beiratzen (beho, d-b, larz), beïratzen (luk), Beïratzen (luk), Beïratzentou (aiz), beiratzentzi (iho), Beirauc (hir), beratzen (garr), Béyratcen (arm), Beyratcen (beh, bard), Beyratzen (a-s), beyratzentzi (d-s)

begiratzaile (54): bégiaçalé (ito), begira salerik (l-o), begiraçaleik (men), begiratçale (ug), begirazaleic (esk), begirazalerik (gar), begirazalez (ürr), béguiaçaleic (alt, etx), béguiaçaleĩc (hau), béguiaçaléik (ath), beguiaçaleric (l-a), béguiaçaleric (z-p), béguiassaléic (irur), béguiassaléric (o-z), beguicaleïc (l-z), beguira zaileric (iri), 
beguiraçalarik (bil), beguiraçale (mar), beguiraçaleïc (anh, pag), beguiraçaleic (mau), beguiraçaleric (ainh, soh, g-i, larr, las, zal), béguiraçalerik (lig), béguirassalé (bun), beguiratcaleric (be-l), beguirazale (ame), beguirazaleik (alo), beïatçaleic (ost), beiraçaileic (a-m), beiraçale (bard), beïracaleïc (aha), béiraçaléic ( $a-z)$, beïraçaleic ( $a-$ zo), béiraçaléïk (beh), beïraçaleric (arm), beiraçalerik (jut, ort), beïratçaleïc (gab), beïratcaleïc (iba), beiratçaleik (d-g), beiratceleric (hir), beiratsaleïc (mend), beiratzale (jats), beiratzaléik (azka), beiratzaleric (beho), beïrazale (garr), beirazaleik (d-b), beyaçaleic (g-zo), beyrazaleek (a-s)

begitarte (1): beguithartia (osp)

behar-1 (203): Beaüc (bai), beauk (d-s), Beaun (bai), Béhaouc (aint), Behaouc (aha), Béhaoun (aint, zar), béhar (ain, aint, zar, a-z, kan, etx, lah, don), Béhar (aint, g-z, iba, ito, l-s, o-z), béharétan (kan), béharciéçu (ath), Béharda (bun), béhardira (bit), béharrac (ain, ain, ain, aint, zar, a-z, a-z, a-z, mug), béharragocoac (ain), béharragouac (zar, a-z), béharraouac (aint), béharrétan (ito), beharro (aha), Béhartcié (ost), Beardiré (ur-p), Behaïci (bard), Behaïcie (gab), Behaïzie (jats), behar (ahe, ahe, ahe, ainh, alt, ame, arh, arn, a-m, aha, beho, eih, azk, azka, altz, altz, lek, bes, d-s, zib, esp, esk, ezt, garr, g-i, get, haz, haz, haz, hen, hoz, iho, iho, ilh, iri, iri, l-b, lak, lak, larz, lar, l-z, luh, meh, leko, men, mug, urd, ort, arro, arro, d-g, hir, zal, bai, suh, u-g, u-h, urr, uzt, mil), Behar (aiz, zar, alo, ame, amo, anh, a-z, arb, arr, arm, ürr, azk, aih, b-l, ban, bas, be-l, bid, bir, soh, sarr, d-b, ünd, etxa, gar, hal, hau, hoz, ida, iru, izp, izt, its, jat, jut, lak, laka, lekb, lig, lan, larr, ald, l-a, luk, mak, mar, mau, mit, ora, izu, arro, s-g, d-l, d-i, sen, sar, zal, dono, dona, dom, irur, urk, ure, uzt, bil, z-p), beharac (azk, azk, azk, lak), beharago (lak, meh), beharagoac (azk), beharagouer (urd), beharaguer (dom), Beharda (osp), (Beharda (urd), behardela (urd), Behardia (arü, hel, müs, pag, zur), Behardira (azka, l-o, mend), Behardire (a-s, bida, meh), Behartci (beh), Behartcié (a-zo), Behartcie (gzo), Behartzi (gam)

behar-2 (96): beharer (urd), beharetan (l-a, pag), beharra (aha, ame), beharrac (aha, arn, eih, zar, ame, ame, arb, azka, ban, d-s, hel, hel, hel, hal, hal, haz, hoz, hoz, iho, iho, iri, iri, jat, jat, lar, lar, meh, meh, meh, leko, mug, arro, bai, bai, bai, ure, uzt, uzt), Beharrac (lar, mug), beharrago (ahe, arb, arr, d-s, zib, g-i, hoz, d-l, uzt), beharragoac (ame, haz, lar), beharragoak (garr), beharragoen (iri), beharragouac (zal), beharragoy (aiz), beharraguac (lan), beharrak (ahe, arb, arb, arr, arr, arr, azka, d-s, d-s, zib, zib, d-b, esp, d-l, d-l, d-l), beharrao (bai), beharraouac (azka), beharrauouac (iho), beharrec (hoz), beharren (d-1), beharretan (arn, izp, mug), Beharretan (ezt, g-i), beharretan_ago (arro), beharretanâo (mug), beharrian (be-l, haz, haz), Beharrian (haz), beharrogouac (hel), beharrunantac (s-g)

behardun (3): behardunak (bida), beharduner (bar), beharunian (osp)

behartsu (4): behartsuagoac (hal), behartsuen (hal, jat), behartuagoac (jat)

behatu (4): Béatcen (a-z), Beatcen (l-b), beatcentci (gab), Beatcentu (ilh)

behatzaile (2): beaçaleic (l-b), beaçaleik (ilh)

behi (152): béhi (ain, aint, zar, a-z, bit, bun, kan, etx, hen, iba, ito, lig, lah, l-s, l-s, ost, ost, o-z, don, ath, irur, ur-p), béhibat (z-p), behi (aiz, ahe, ainh, ame, amo, anh, arb, a-m, aha, arn, beho, eih, arr, arh, arm, ürr, ürr, azk, azka, azka, altz, aih, beh, b-l, ban, bar, bard, bas, be-l, bid, bida, bir, lek, bes, d-s, buz, g-z, soh, sarr, zib, d-b, ünd, esk, ezt, etxa, gab, gam, gar, garr, g-i, get, hel, hal, haz, hau, hoz, ida, iho, iho, ilh, iri, iru, izp, 
izt, izt, its, jat, jats, jut, l-b, lak, laka, lekb, lan, larz, larr, las, ald, osp, l-z, l-a, luh, luk, mak, mau, meh, leko, men, mend, mit, mug, müs, ora, ort, izu, izu, pag, arro, s-g, d-l, d-i, sen, hir, sar, sar, zal, zur, zur, dono, bai, dona, dom, suh, u-h, urk, ure, urr, uzt, mil, bil), Behi (iri), behibat (alt, alo, arü, a-s, esp, lar)

behin (8): Béhin (ain), behin (arm, hir), Behin (d-s, haz, iho, urd), bein (urk)

behiño (6): behiñobat ( $\mathrm{u}-\mathrm{g}$ ), behigno (l-o), behignoat (a-zo, g-zo), behignobat (mar, d-g)

behitto (1): behitto (urd)

behizain (1): behizagnez (alo)

beihala (1): beiyala (ur-p)

beila (14): béilan (kan), beilan (lek, buz, d-b, haz, iho, mil), beilla (ald), beillan (altz, bir, etx, ida, ilh, l-z)

beilatu (35): béillatçènn (bit), beïlatcen (arm, dona), beilatcen (its, mak), beilatzen (urk), beilatzenzi (garr), beillatcén (iba, ost, don), beillatcen (ainh, a-m, bard, be-l, soh, jut, l-b, larr, las, mau, mend, arro, dono, u-g, z-p), beillatcendu (d-g), beillatsën ( $g$ z), beillatsen (l-s), beillatzen (alo, beho, gam, jats), beillazen (ur-p), bellatzen (l-o), beyatcen (g-zo)

beilheratz (3): beilheratz (arro), beleatz (men, zal)

belar (148): bélhar (lah), bélharac (kan), bélharra (ain, zar, bit, l-s, don), bélharrac (ito), belarra (bid, bir), belhar (haz, iru), belhara (alo, a-m, g-z, etx, etxa, hau, lak, l-z, l-a, mar, müs, o-z, d-g, zal, dom, bil), belharac (azk, ida, lig, luh, mau, irur), belharra (aha, aiz, ainh, ame, amo, a-zo, arh, beh, bard, beho, be-l, bes, bun, buz, eih, g-zo, soh, d-b, esk, ezt, gab, gam, gar, g-i, hel, hen, hoz, ilh, jut, l-b, laka, larz, lar, osp, luk, mak, mend, mug, ora, ort, izu, pag, arro, s-g, d-i, bai, dona, ath, u-g, u-h, urk, ure, mil, z-p), Belharra (leko), belharrac (aint, alt, anh, arn, a-z, arb, arr, arm, azka, aih, b-l, ban, lek, ünd, get, hal, haz, iba, iho, iri, iru, izp, izt, its, jat, jats, lekb, lan, larr, las, ald, meh, men, urd, ost, sen, hir, dono, suh, ur-p, urr, uzt), belharrak (ahe, arü, ürr, a-s, altz, bar, bida, d-s, sarr, zib, garr, l-o, mit, d-l, sar, zur)

belaunikatu (13): belhaünicatou (lekb), belhaünicatu (jats), belhaïcatau (o-z), belhaïcatu (lig, irur), belhaounicatou (aint, zar), belharicatzen (urd), belharricatu (larr), belhauncatu (aih), belhaunicatu (d-s, izu), belhaunikatu (garr)

belauniko (136): bélhaïco (l-s), bélhaounico (ain, bit, ost), bélhaounka (kan), bélhaouriko (beh), beelhaunico (get), behaünca (gab), behaüniko (arb), belaüco (dom), belaunico (azk, bid), belauniko (leko), belhaüco (mug), belhaügniko (d-g), belhaünca (ure), belhaünica (u-h), belhaünico (anh, arm, lek, haz, luk, bai), belhaüniko (aiz, ahe, arr), belhaünko (d-b), belhaïco (ainh, alt, a-zo, g-z, g-zo, ünd, etx, hau, ito, l-b, l-z), belhaiko (altz, sarr, mit), belhaico (osp, ath), belhaiko (alo, ürr, bar, laka, l-o, men), Belhalaco (d-1), belhaoünico (bun, hen), belhaouco (hel), belhaounica (ora), belhaounico (aha, arh, buz, jut, sen, dona), belhaouniko (a-z, gam, don), belharico (arü, esk, etxa, g-i, ida, l-a, mar, mau, müs, arro, s-g, zal, z-p), belhariko (gar), belharrico (be-l, soh, pag), belhau'nico (ban), belhauco (ilh), belhaunco (bes), belhaunica (iho, d-i), belhaunico (a-m, ame, amo, arn, beho, b-l, bas, bir, eih, ezt, hal, hoz, iba, iri, iru, izp, its, jat, lak, lah, lan, larz, lar, las, ald, luh, mak, ort, hir, dono, suh, u-g, ur-p, urr, uzt), belhaunika (a-s, mil), belhauniko (azka, bida, zib, esp, mend, d-l, sar, zur), belhauninco (izt), belhaunka (meh), belhaunku (urk), belhayko (bil), belhoinko (bard)

beldur (214): béldour (ain, ain, don), béldourac (kan), béldourra (bit), béldourrac (ain, ost), 
béldourrak (don), béldourrez (hen), béldures (l-s), béldurrac (ito), béldurtçu (ost), bedur (its), beldour (aha, aint, eih, zar, arh, arm, a-s, bit, bun, buz, kan, hel, jut, lah, ald, luh, ora, sen), beldoures (luh), beldourra (hen), beldourrac (aha, eih, zar, arm, azka, buz, hel, iba, jut, lekb, lah, sen, dona), beldourrak (gam), Beldourrak (a-s), beldourtçou (iba), beldourtzou (gam), beldu (ur-p), beldur (aiz, ahe, ahe, a-m, ame, anh, arn, a-z, arb, a-zo, arr, aih, aih, beh, beho, b-l, ban, bard, bas, bid, bir, lek, bes, g-zo, zib, d-b, esp, ezt, get, hal, haz, haz, hoz, ilh, iri, izp, izt, jat, jats, l-b, lak, lekb, lan, larz, lar, las, l-o, luk, mar, mau, meh, leko, mug, ort, izu, d-g, d-i, hir, sar, zur, dono, bai, dona, dom, suh, u-g, u-h, urk, urr, uzt, mil), beldura (azk, urd), beldurac (azk, lak, mar, meh, dom), beldurak (l-o), beldurçu (mend), beldurez (d-s, lig, irur), Beldurez (ur-p), beldurra (ame, amo, azka, get, mak, ure), beldurrac (a-m, amo, arn, a-z, a-zo, beho, b-l, ban, bas, bid, bes, g-zo, ezt, gab, hal, iho, iru, izt, its, jat, jats, larz, las, ald, mak, leko, mend, ora, urd, ort, izu, d-g, d-i, dono, bai, suh, u-g, u-h, ure), Beldurrac (bir, luk), beldurraicie (gab), beldurrak (aiz, arb, arr, bard, bida, zib, d-b, esp, garr, ilh, l-b, d-l, sar, zur, urk, mil), beldurraz (lan), beldurres (bun), beldurrez (beh, lek, hoz, izp, lar, uzt), Beldurrez (iri, hir), beldurtzu (d-s, iho), beldurzu (garr)

beldurkunde (1): beldurkundeac (mug)

beldurtasun (1): beldurtasunaz (urr)

ber (26): ber (altz, g-i, larr, osp, müs, zal), Ber (alt, altz, esk, etxa, g-i, lig, lig, osp, l-z, zal, irur, irur), ber_denboran (bar), Ber_denboran (altz)

Ber gisa (13): bergaiça (bil), bergijan (men), bergisan (mit), berguisan (etx, gam, z-p), ber guizan (lig, irur), ber guisan (g-i, müs, l-z, zal), ber gisala (esk)

bera (182): bé (altz, hau, hau), béac (etx, hau, l-s), béak (ath), béra (ost), bérac (ain, aint, zar, alt, a-z, bit, kan, iba, ito, z-p), bérén (ost), béren (zar, a-z, iba, z-p), bérenn (bit), be (ath), beac (g-i, laka, l-z, o-z), beak (altz), Beaouc (zar), Beaouk (mend), bera (ahe, arm, arm, aih, ban, bar, lek, its, lekb, l-a, mak, urd, arro, d-l, sen, hir), berac (ainh, a-m, ame, anh, arn, a-zo, arü, ürr, azka, aih, b-l, ban, bard, bas, lek, bes, g-zo, sarr, ezt, etxa, gab, hal, ida, iho, iho, iri, izp, izt, its, jat, lig, larz, larr, lar, las, osp, l-a, mak, meh, mit, mug, müs, urd, izu, s-g, d-g, hir, hir, zal, dono, dom, irur, u-g, urk, ure, urr), beracen (mug), berak (aiz, ahe, ahe, a-s, bar, bida, d-s, zib, zib, esp, gam, gar, garr, l-o, leko, men, ora, d-l, don, mil, bil), beran (ünd), beras (ban), Beras (luh), berec (ora, s-g), berei (hal, jat), beren (ainh, ame, ame, amo, anh, arm, arü, ürr, a-s, azka, azka, beh, bar, bir, sarr, ünd, esp, gab, gar, garr, get, hal, hoz, iho, iru, its, jat, jats, jut, osp, l-a, luh, luk, mak, meh, mug, müs, s-g, sen, bai, dona, suh, ure, mil), berenaz (zib), berenik (sar), berer (ure), berez (bida)

berdin (37): bardin (gar, luk, mau, hir, ath, urk, uzt, mil), Bardin (arü, bar, sarr, ünd, izt, mug), Bérdin (don), berdin (a-m, ame, bas, hel, jut, lekb, lan, las, mend, ort), Berdin (aint, arm, arn, beh, b-l, bun, eih, gab, leko, d-g, u-g, zar)

bere (278): béé (alt, o-z, ath), bée (etx, etx), béré (ain, aint, zar, zar, a-z, a-z, bit, bit, bun, bun, kan, g-z, g-z, hen, hen, iba, izt, ito, l-b, lig, lig, lig, lah, lah, l-s, mau, ost, ost, don, ath, irur, irur, irur, z-p, z-p), bére (mit, ur-p), bee (l-z, l-z, leko, urk, urk, urk), beré (arm, l-b), bere (a-m, aha, arn, beho, aiz, aiz, ahe, ainh, alo, alo, ame, ame, amo, amo, anh, anh, arb, arb, a-zo, arr, arr, arh, arh, arm, arü, arü, ürr, ürr, a-s, a-s, azk, azk, azka, aih, beh, b-1, b-l, ban, ban, bar, bar, bard, bard, bas, bas, bas, be-l, be-l, bid, bid, bida, bida, bir, bir, lek, bes, bes, d-s, buz, eih, g-zo, soh, soh, sarr, sarr, zib, zib, zib, zib, zib, d-b, 
ünd, ünd, esp, esp, esk, esk, ezt, ezt, etxa, gab, gab, gam, gam, gam, gar, gar, garr, g-i, g-i, get, get, hel, hel, hal, hal, haz, haz, hoz, ida, ida, ida, iho, iho, ilh, ilh, iri, iru, iru, izp, izp, izt, izt, its, its, jat, jat, jats, jats, jut, jut, lak, lak, laka, laka, lekb, lig, lan, larz, larz, larr, lar, lar, las, ald, osp, osp, l-a, l-a, l-o, l-o, luh, luk, luk, mak, mak, mar, mar, mau, meh, meh, leko, leko, men, mend, mend, mit, mit, mug, müs, müs, ora, ora, ora, urd, urd, ort, ort, izu, izu, pag, pag, pag, arro, arro, s-g, d-l, d-l, d-l, d-l, d-g, d-g, d-i, sen, sen, sen, hir, hir, hir, sar, zal, zal, zur, zur, dono, dono, bai, bai, dona, dona, dom, suh, suh, u-g, u-g, u-h, u-h, ure, ure, urr, urr, uzt, uzt, mil, mil, bil, bil), beyan (ath)

berean (71): beh (urk), Ordou_berian (buz), béréan (mug), bérian (aint, zar, a-z, kan, iba, urp, ur-p), bériann (bit, bit), berïn (arro), berean (ahe, bida, zib, zib, get, haz, iru, its, its, d-l, d-i, sen, hir, sar), berian (aiz, ame, ame, arb, arr, arh, arh, azka, ban, beho, bid, bir, lek, d-s, buz, hal, hoz, iho, iru, jat, jats, lak, lekb, lekb, larz, las, l-o, luh, luh, luh, mar, meh, ora, izu, d-g, dono, bai, dona, dom, u-g, u-h, u-h, mil)

berehala (130): béhala (zar, alt, a-z, iba, l-s), Béhala (aint, beh, iba, ost), béian (ath), Béréala (hen), béréhala (ain, don), Béréhala (don), bérhala (bun, l-s), Be'ehala (ban), beahala (d-s), behaala (leko), Behaala (bes, leko), behala (aha, aiz, ainh, alo, amo, a-m, a-zo, arm, arm, arü, altz, beh, be-l, g-zo, g-zo, soh, d-b, etx, gab, garr, g-i, larz, larr, l-z, men, o-z, pag, pag, zal, dona, irur), Behala (arm, aih, bard, gam, ilh, jats, jut, l-b, larr), behâla (zur), Bereala (bida, bai), berehala (arn, azk, bid, bida, eih, lek, zib, hal, iri, izp, izt, jat, lan, lar, lar, meh, meh, ort, hir, hir, sar, sar, dono, urr, urr), Berehala (anh, arn, azk, zib, esp, gab, iho, izp, izt, lan, ald, mak, mend, zur, dono, urr, uzt), berhala (etxa, gar, ida, ida, osp, l-a, l-o, mar, mar, mau, müs, urd, s-g, bil), Berhala (l-a, müs, z-p), berheala (bas, hel, hoz, suh), Berheala (hel, suh), Berreala (bas)

beren (12): béren (aint), beren (a-m, aha, arn), been (altz, altz, bes, zur), Been (altz), ben (alo, ur-p, ur-p)

bero (1): beroa (zib)

beroki (1): beroki (ahe)

berotu (141): béotcén (hau), béotcen (alt, a-z, ath), béotsen (etx, l-s), bérocen (ur-p), bérotçènn (bit), bérotcén (ain, ost), bérotcénn (hen), bérotcen (aha, aint, zar, beh, z-p), bérotsén (ito), bérotsen (bun, kan, lig), bérotzen (lah), beotcen (altz, bard, gab, g-i, ida, l-z, mar, izu, pag), beotciaequin (g-zo), beotsén ( $g-z)$, beotsen (o-z), beotzen (hel), beratcen (l-b), berocen (bir), berotcén (iba), berotcen (ainh, a-m, amo, anh, arn, arb, arr, arm, arü, azk, b-l, be-l, bid, bida, eih, lek, soh, sarr, ünd, esp, etxa, get, hal, hoz, ilh, izt, its, jut, laka, larr, las, ald, osp, mak, mau, meh, leko, men, mend, mug, müs, ora, ort, arro, s-g, d-g, d-i, sen, hir, zal, dono, dona, suh, u-g, ure, urr, uzt, bil), berotciaikin (a-zo), berotsen (arh, ban, irur, u-h), berotzen (aiz, alo, ame, beho, ürr, a-s, azka, aih, bas, bes, d-s, buz, zib, d-b, esk, ezt, gam, gar, garr, haz, iri, iru, izp, jat, jats, lak, lan, larz, l-a, l-o, luk, mit, d-l, sar, zur, bai, dom, urk, mil), berrotzen (lekb)

berri (3): berri (a-m, ur-p, urr)

berriz (3): bériz (irur), beriz (lig), berriz (b-l)

berrizahar (2): berrichaharra (arm, arm)

Bertan (11): bertan (ainh, ürr, bar, esk, g-i, lig, mit, s-g, ath), bertann (hau), berthan (pag) bertsu (2): bertsuan (d-1), pertsuan (zib)

bertze (138): bértcé (ain, ain, don), bértcébati (don), beceatetan (bard), berçe (ur-p), berçeic (ur-p), berce (azk, azk, b-l, bir, bir, iri, iri, leko), berceat (leko), berceic (bir), bercian 
(ur-p), bersia (ban), bertcé (aint, zar, hen), Bertcé (don), bertcétann (hen), bertce (aint, ame, anh, anh, arb, arb, arn, arr, b-l, b-l, bida, eih, lek, lek, lek, esp, get, get, get, get, izt, izt, its, its, its, its, lar, lar, ald, ald, mak, mak, meh, ort, ort, d-g, d-g, sen, sen, hir, dona, dona, suh, u-g, u-g, ure, ure, ure, urr, urr, uzt, uzt), Bertce (esp, hir, urr), bertcebat (mug), bertcebatçûtan (mug), bertcebatian (esp), bertcek (meh), bertcera (lar), bertcetan (mak), bertsé (kan, kan), bertse (arh, luh, luh, luh), bertzé (lah, lah), bertze (aiz, ame, ame, arn, arr, arh, aih, aih, bes, bes, zib, zib, zib, hal, hal, haz, haz, haz, iru, jat, jat, lak, lak, lak, ort, d-l, d-l, d-l, zur, zur, bai, bai, mil, mil), Bertze (zib), bertzean (sar), bertzeari (ahe), bertzeatian (bas), bertzeic (aih), bertzetan (sar, zur), bertzietan (bas), berze (bid, bid)

bertzorduz (11): bertcé ordouz (don), berce ordus (b-l), bertce ordus (ure), bertce orduz (bida), bertze orduz (zib), bertce orduz (get), bertce orduz (its), bertze orduz (lak), bertce orduz (mak), bertze orduz (ort), bertce orduz (urr)

beste (197): béjté (ost), béssté (bit, bit), bésste (bit), bésté (bun), béstéékin (ito), béste (l-s), bejte (iba, iba, ost), besté (a-z, a-z, hau, hau, ito, l-s, o-z, ath), Besté (altz), bestéic (ito), bestéat (ito), bestébat (bun, irur), bestéic (ainh), bestéik (ath), bestélibatetan (o-z), beste (aha, ainh, a-m, alo, amo, amo, a-z, a-zo, a-zo, arh, arm, arm, ürr, ürr, a-s, a-s, a-s, azka, azka, azka, altz, aih, bar, beho, be-l, be-l, d-s, buz, buz, g-zo, soh, soh, d-b, d-b, ünd, ezt, ezt, etx, etx, gam, gam, g-i, hoz, hoz, ida, iho, ilh, iru, izp, izp, jats, jats, jut, jut, l-b, l-b, laka, laka, lekb, lekb, lan, lan, larz, larz, larr, larr, larr, las, las, osp, osp, osp, l-z, l-z, l-a, l-o, l-o, luk, luk, mau, men, men, men, mit, mit, müs, müs, ora, ora, ora, urd, urd, izu, izu, pag, arro, arro, s-g, s-g, d-i, d-i, zal, dono, dom, dom, suh, u-h, u-h, u-h, bil, bil, z-p), Beste (a-zo, bar, g-zo, hoz, urd), besteic (hau, lig), besteat (d-s, g-zo), besteatçutan (mend), besteatcietan (gab), besteatian (gab), besteatzuetan (hel), bestebat (alo, arü, esk, g-i, l-a, pag, z-p), bestebatetan (ida, bil), bestebatçouetan (aha), bestebati (gar), bestebatian (mar, mar), besteic (ünd), besteik (arü, altz, bar, sarr), bestelibati (gar), besten (altz), besteric (esk, ida, iho, izp, mau, zal), besterik (bar), bestetan (dono), bestiac (alt), bestian (hel, mend), beze (bard), bezeatetan (urk), bezetan (urk), bezté (beh), beztéïc (irur), beztétan (beh), bezte (etxa, garr), bezteat (garr), beztebat (lig)

bestorduz (23): Béstordus (ito), besté_orduz (a-z), besteorduz (urd), beste_orduz (ezt, etxa, gab, ilh, osp, s-g), bestordous (a-zo, pag), bestordouz (buz, gam), bestordus (altz, mar), bestorduz (amo, g-zo, lan), Bestorduz (izp), beste ordous (ora), beste ordouz (a-s), besté ordus (hau)

bet-betan (5): bet betan (haz), Bet betan (lek), Betbetan (zib, lar, ort)

bete (2): bethe (ame), betheric (mar)

beti (272): béthi (ain, aint, alt, a-z, a-z, bit, bit, bit, kan, kan, etx, lah, lah, l-s, ost, ost, don, don, ath), Béthi (iba), bèti (hen), béti (hen, ur-p), bethi (aiz, aiz, zar, zar, ahe, ahe, ainh, ainh, alo, alo, a-m, aha, arn, beho, eih, ame, ame, amo, amo, anh, anh, arb, arb, a-zo, arr, arr, arh, arh, arm, arü, ürr, ürr, a-s, a-s, azk, azk, azka, altz, beh, beh, b-l, b-l, ban, bar, bar, bard, bard, bas, be-l, be-l, bid, bid, lek, lek, bes, bes, bun, d-s, d-s, buz, buz, g-z, g-zo, g-zo, soh, soh, sarr, d-b, d-b, ünd, esk, esk, ezt, ezt, etxa, etxa, gab, gab, gam, gar, gar, garr, g-i, g-i, get, get, hel, hel, hal, hal, haz, haz, hau, hau, hoz, hoz, ida, iho, iho, ilh, ilh, iri, iri, iru, izp, izp, izt, izt, its, its, jat, jat, jats, jats, jut, jut, l-b, l-b, lak, lak, laka, laka, lekb, lig, lig, lig, lan, lan, larz, larz, larr, larr, lar, lar, las, las, ald, osp, osp, l-z, 
l-a, l-a, l-o, l-o, luh, luk, mak, mak, mar, mar, mau, mau, meh, meh, leko, leko, men, men, mend, mend, mit, mug, mug, müs, müs, ora, ora, urd, urd, ort, ort, izu, pag, pag, arro, arro, arro, s-g, s-g, d-l, d-g, d-g, d-g, d-i, d-i, sen, hir, hir, sar, sar, zal, zal, zur, zur, bai, bai, dona, dona, dom, dom, suh, suh, ath, irur, irur, irur, u-g, u-g, u-h, u-h, urk, ure, ure, urr, urr, uzt, uzt, mil, mil, bil, bil, z-p), Bethi (aih, bar, bida, zib, zib, esp, esp, etx, lan, l-z, urr), beti (arm, bir, dono, dono, u-g), bethi (mit)

betiri (10): bethiri (dom) bethiriekin (dom), betiri (lar, mar, leko), betiriikin (leko), betirirekin (lar. mak), bettiri (izt), bettirirekin (izt)

bezain (10): béçaïn (a-z), beçagn (altz), beçain (izt), becen (lek, hir, dono), becin (bida), bezaïn (garr), bezan (dom), bezen (urk, mil)

bezain bat (1): bezain bat (beho)

bezala (4): beçala (ida), becala (d-g), bezala (arn, zib)

bezalakoa (1): beçalacoa (hir)

bi (440): bi (aha, aiz, aiz, aiz, aiz, ain, ain, ain, zar, zar, zar, ahe, ahe, ahe, ainh, ainh, ainh, aint, a-m, alt, alt, alt, alo, alo, alo, ame, ame, amo, amo, amo, anh, anh, anh, arn, a-z, a-z, a-z, arb, arb, arb, a-zo, a-zo, a-zo, arr, arr, arr, arh, arh, arh, arm, arm, arm, arü, arü, arü, ürr, ürr, ürr, a-s, a-s, a-s, azk, azk, azk, azka, azka, azka, altz, altz, aih, aih, beh, beh, beh, beho, b-l, b-l, b-l, ban, ban, ban, bar, bar, bar, bard, bard, bard, bas, bas, bas, be-l, be-l, be-l, bit, bit, bit, bid, bid, bid, bida, bida, bida, bir, bir, bir, lek, lek, bes, bes, bes, bun, bun, bun, d-s, d-s, d-s, buz, buz, buz, eih, kan, kan, kan, g-z, g-z, g-z, g-zo, g-zo, g-zo, soh, soh, soh, sarr, sarr, sarr, zib, zib, zib, d-b, d-b, d-b, ünd, ünd, ünd, esp, esp, esp, esk, esk, esk, ezt, ezt, ezt, etx, etx, etx, etxa, etxa, etxa, gab, gab, gab, gam, gam, gam, gar, gar, gar, garr, garr, garr, g-i, g-i, g-i, get, get, get, hel, hel, hel, hal, hal, hal, haz, haz, hau, hau, hau, hen, hen, hen, hoz, hoz, hoz, iba, iba, iba, ida, ida, ida, iho, iho, iho, ilh, ilh, iri, iri, iri, iru, iru, iru, izp, izp, izp, izt, izt, izt, ito, ito, ito, its, its, its, jat, jat, jat, jats, jats, jats, jut, jut, jut, l-b, l-b, lak, lak, laka, laka, laka, lekb, lekb, lekb, lig, lig, lig, lah, lah, lah, lan, lan, lan, larz, larz, larz, larr, larr, larr, lar, lar, lar, l-s, l-s, l-s, las, las, las, ald, ald, ald, osp, osp, osp, l-z, l-z, l-z, l-a, l-a, l-a, l-o, l-o, l-o, luh, luh, luk, luk, luk, mak, mak, mak, mar, mar, mar, mau, mau, mau, meh, meh, meh, leko, leko, leko, men, men, men, mend, mend, mend, mit, mit, mit, mug, mug, müs, müs, müs, ora, ora, ora, urd, urd, urd, ost, ost, ost, o-z, o-z, o-z, ort, ort, ort, izu, izu, izu, pag, pag, pag, arro, arro, arro, s-g, s-g, s-g, d-l, d-l, d-l, d-g, d-g, d-g, d-i, d-i, d-i, sen, sen, sen, don, don, don, hir, hir, hir, sar, sar, sar, zal, zal, zal, zur, zur, zur, dono, dono, dono, bai, bai, bai, dona, dona, dona, dom, dom, dom, suh, suh, suh, ath, ath, ath, irur, irur, irur, u-g, u-g, u-g, u-h, u-h, u-h, urk, urk, urk, ure, ure, ure, ur-p, ur-p, ur-p, urr, urr, urr, uzt, uzt, uzt, mil, mil, mil, bil, bil, bil, z-p, z-p, z-p), (bi (ito), Bi (luh), bo (mug)

bidaiant (135): bïaiant (zib), bïaiantek (zib), biayant (a-z), biayanteï (a-z), bidaïant (don), bidéïant (ito), bidageant (arro), (bidairantak) (bida), bidairante (bida), bidajant (bar, be-l, esk, etx, gar, lak, laka, lig, l-z, men, urd, zal, ath, irur), bidéjant (hau, o-z), bidajentec (ainh), bidayant (aiz, ainh, ame, ame, a-zo, ürr, a-s, b-l, d-s, g-zo, soh, d-b, hoz, iho, ilh, jut, l-b, larz, ora, ort, izu), bidayènt (bit), bidéyant (kan), bidéyantéc (kan), bidayantec (a-m, arn, beho, dona, iru, lan, meh), bideant (d-l), bideantek (ahe), bidejan (mit), bidejant (alo, arü, altz, sarr, ünd, g-i, osp, mau, müs, pag, s-g, bil, z-p), Bidejanten (altz), bidejent (larr), bidejente (l-a), bideyant (b-l, lekb, l-o, 
ure), bideyante (its), bideyent (d-i), bidjant (ida), bijéjant (alt), piaïant (ain, dom), Piaiant (lek), piaiantek (buz), piaient (bir), piaiyant (ost), piaiant (bard, lek, hel, lah), piajiante (ban), piayant (azk, azk, azka, aih, aih, bas, bes, esp, gab, gam, luk, leko, sen, suh), piayantec (iri, mend, bai), piayanthe (beh), piayyantec (arm), pidajantac (izp), pidayant (anh, jats, las, ald, mak), pidejantec (arh), pideyantec (u-h), pieyjant (mil), pirayant (luh, luh, luh), piyayant (arb, arr, bid, get, mug, dono, uzt), piyayantec (hen), pyayetaco (urr), bidayantec ()

bidaiatzaile (1): piayazaillé (ur-p)

bide (2): bidea (zib, d-l)

bidekari (2): bidekariac (arb), bidekariek (arr)

bidelari (3): bidelari (sar, zur), Bidelarien (sar)

bidezko (2): bidezco (hal, jat)

biga (1): Biga (jut)

biharamun (148): Bihaamün (d-b), Bihaamoun (aha, hel, ito), Bihaamoune (ost), Bihaamun (bes, leko), Bihamën (g-z), Bihamén (hau), Bihamen (alo, arh, arü, bar, g-zo, sarr, ünd, lekb, osp, l-a, l-o, müs, urd, o-z, pag, ath, urk, bil, z-p), Bihamenen (irur), Bihamenian (alt, etx, l-z), Bihamenin (altz), Bihamon (a-zo), bihamoun (iba), Bihamoun (aint, a-z, arm, azka, ban, bard, bun, gam, ilh, jats, l-b, larz), Bihamounian (aiz, garr), Bihamun (amo, beh, beho bas, bir, esp, haz, l-s, men, mend, ort, izu, d-g, d-i, dom, ur-p), Bihâmun (ainh, zur, bai), biharamen (be-l), Biharamen (ürr, kan, esk, etxa, gar, g-i, ida, laka, lig, mar, mau, arro, u-h), Biharamenian (soh), Biharamoun (ain, eih, zar, a-s, bid, buz, hen, iru, jut, lah, ald, luh, ora, sen, don, dona), Bihéramounn (bit), Biharamun (ahe, a-m, ame, anh, arb, arn, arr, azk, aih, b-l, bida, lek, zib, ezt, gab, hal, hoz, iho, iri, izp, izt, its, jat, lan, las, luk, mak, meh, mit, mug, s-g, d-l, hir, sar, dono, suh, u-g, ure, urr, uzt, mil), Biharamunean (get), Biharamunian (d-s, lar), biheramen (larr), Biheramen (zal), Biheramun (lak)

bihi (30): bihi (arh, kan, gam, iri, izt, larz, ald, meh, ost), Bihi (meh), bihibat (bard, d-b, get), bihic (bir, lek), bihien (arb, arr), bihiic (bas), bihik (zur, urk), bihiric (aih, lah, dono, dona, dom), bihirik (arb, arr, d-l, d-g, mil)

bihotz (8): bihotx (lar), bihotz (bida, bida, garr, sen, hir), Bihotz (bida, sen)

bila (1): billa (zib)

bildu (51): bil (arü, altz, bar, be-l, g-z, soh, sarr, ünd, larr, osp, müs, pag, arro, zal, ath, z-p), bilartio (ainh, urd), bildiac (lak), bildiak (d-b), bildou (ain, arm, lekb, lah), bildu (amo, aih, beh, b-l, bir, g-zo, iho, iru, lan, izu, suh, ure, urr), bildua (hal, jat), bilduac (izt, meh), bilduic (a-m, l-z), bilduik (etx, men, urk), bilduric (l-a, s-g), bildurik (alo)

bisaia (2): bisaia (sar), bisaya (ezt)

bista (17): bichtarat (iho), bichtatic (aha, a-m, anh, a-z, ban, bid, iho, jut, lan, ora, bai), bistatic (mak, mug, müs), bistatik (d-l), biztatik (sar)

bitarte (2): bitartian (hal, jat)

bizi (7): bici (altz, iri, lah, hir), (bicitcen (bida), bisi (l-s), bizi (garr)

biziki (72): bicik (mug), biciki (beho, zar, arh, arh, arm, beh, beh, b-l, lek, buz, iba, ilh, izt, ito, ito, l-b, lah, las, mak, mar, leko, mend, ora, ost, izu, d-g, don, don, dona, u-g, u-h, u-h, ure), Biciki (gab, ost), biciqui (amo, a-zo, a-zo, eih, g-zo, jut), Biciqui (a-zo, g-zo), bisiki (kan), bisiqui (l-s, l-s), bissiki (aint, bun, bun, l-o, pag), biziki (aiz, a-s, azka, bes, d-b, ezt, gam, garr, hel, lak, lekb, lekb, larz, larz, luk, dom), Biziki (d-b, larz) 
bizkar (1): bizkar (larz)

bizkitarte (2): bizkitart (d-1), Bizkitartean (iri)

boiajur (9): bogajür (garr), bogajur (garr), boyageurs (mar), boyajur (urk), boyajurrac (bun), vouaïayur (1-s), voyajur ( $g-z$, etxa, iba)

borda (1): bordan (sen)

borondate (1): borondatez (ame)

borta (267): borta (aiz, anh, beh, bid, lek, jats, luh, luh, men, mend, mit, don, dono), Borta (aiz, ban), bortain (jats), bortaatetan (urk), bortabat (arü), bortaen (beh), bortain (bid), bortan (uzt), bortaren (ame, lek, haz, l-o, men, dono), borteatian (aiz), bortha (a-m, aha, aint, beho, eih, zar, ainh, alt, alo, ame, amo, a-z, arh, arm, ürr, a-s, azka, altz, aih, b-l, bar, bar, bas, be-l, bit, bes, bes, bun, bun, d-s, buz, kan, soh, sarr, d-b, ünd, esk, ezt, etx, etxa, gam, garr, g-i, hau, hoz, iba, ida, iho, ilh, iri, iru, izp, izt, ito, ito, its, jut, l-b, lak, laka, lekb, lig, lah, lan, larz, larr, l-s, las, ald, osp, l-z, l-a, l-o, luk, mak, mar, mug, müs, ora, urd, ost, o-z, izu, pag, arro, s-g, d-g, d-g, d-i, zal, dona, dom, suh, suh, ath, u-g, u-h, mil, bil, z-p), Bortha (a-zo, mau, irur), borthaaan (hel), bortha-an (aih), borthaïn (aint, a-z, a-zo, bas, bit, gab, gam, iba, l-b, l-s, ost), borthaën (hau), borthaén (g-z), borthaat (g-zo, leko), borthaatian (gab, hel), borthabatetan (bard), borthabati (gar), borthaen (zar, arü, g-zo, sarr, d-b, ünd, lig, osp, l-z, o-z, pag, ath, irur), borthain (azka, altz, ilh, jut, larz, mend, mend, izu), borthan (bard, etx, ito, urk, urk), bortharén (don), bortharen (ainh, alt, alo, amo, anh, arh, arm, beho, eih, ürr, a-s, b-l, be-l, d-s, buz, kan, soh, esk, ezt, ezt, etxa, gar, g-i, hoz, ida, iho, iri, iru, izp, izt, its, lak, laka, lekb, lah, lan, larr, las, l-a, luk, mak, mar, mau, leko, mit, mug, müs, ora, urd, arro, s-g, d-g, d-i, zal, dona, dom, suh, u-g, u-g, u-h, mil, bil, z-p), borthat (garr), borthiaan (bes), borthian (alo), borthien (altz), bortian (g-z, mar, o-z), bortiari (l-a), bortietaco (arm, esk, gab, gab, zal, irur), Bortietaco (arm), bortietako (l-o), bortin (arü, sarr, ünd), bothaan (bas)

bortitz (7): borthitcénéz (ain), borthitcez (azk), borthitz (altz, arn, bes, iri), borthitzez (larz) bortizki (4): borthiski (altz, beho), borthizki (azka), bortiski (mak)

bortu (40): bort'ondoko (bar, bar), borthu (ame, ame, a-zo, esk, o-z, s-g), Borthu (g-zo), Borthuetaco (bas), bortitaco (esk), bortou (arh, ora), bortu (a-zo, altz, g-zo, g-i, laka, lig, lan, mit, müs, o-z, arro, s-g, zal, irur, u-h, bil), Bortu (lar, l-a, arro), bortubatetan (gar), bortuco (etxa, g-i, g-i), Bortuco (etxa), bortuetaco (hel), Bortuetaco (hel), Bortuko (1-o)

bortxatu (1): bortchatuac (hel)

bortxaz (1): Bortchaz (bil)

botxe (18): botche (amo, larr, osp, l-a), botchebaten (l-o), botchu (alt, esk, etx, gar, g-i, hau, laka, l-z, arro, s-g, zal, ath)

brau (1): braou (kan)

brillanki (1): brillanki (gab)

brillant (2): brillant (beho), brillantic (bit)

brillantzia (2): brillantsia (irur), brilllantsia (lig)

buru (224): buia (alt, g-z, etx, hau, l-z, o-z, ath), buiac (etx, l-z), buila (men), büria (d-b), bouria (aha, aint, arm, a-s, bit, bun, eih, zar, gam, iba, lekb, ald, ost, don), bourian (aha, bun), bouriane (ost), bouriari (a-s), bourou (buz, kan), bouroua (ain, hen, sen, dona), bourouan (arm, lah), bourouann (hen), bourouia (lah), bouroura (iba), 
bourourat (ald), bourous (arh, kan), bourouz (a-s, hel, iba, lekb), bourria (ora), bourua (hel), buüs (alt, o-z), buria (aiz, ainh, alo, a-m, amo, amo, anh, a-z, a-zo, arh, arü, ürr, azka, aih, ban, bard, beh, beho, bid, d-s, g-zo, sarr, ünd, esk, etxa, gab, gar, garr, g-i, iho, ilh, izp, ito, jats, jut, l-b, lak, laka, lig, lan, larz, larr, l-s, las, l-a, l-o, luk, mar, mau, men, mend, mit, müs, urd, izu, arro, s-g, zal, bai, dom, suh, irur, u-h, bil, z-p), buriac (ida, pag), burian (amo, azka, ban, d-s), burien (bard), burin (arü, sarr, ünd), buru (arn, be-l, lek, soh, izt, lar, hir, zur), burua (ahe, ame, bida, bir, zib, esp, get, haz, iri, iru, jat, leko, ort, d-l, d-g, dono, u-g, ure, ur-p, urr, uzt, mil), buruac (azk, b-l, hal, lar), buruan (lek, haz, its, lar, urr, mil), buru-buruan (sar), buruik (urk), buruin (urk), burura (a-z, d-b, izp, d-l), bururaino (iri), bururat (arb, arr), burus (arü, sarr, ünd, l-a, $\mathrm{s}-\mathrm{g}, \mathrm{u}-\mathrm{h}$ ), buruya (arb, arr, bas, bes, its, mak, meh, mug), buruyan (meh), buruz (ahe, ainh, alo, anh, a-z, bas, be-l, bid, bida, lek, d-s, soh, esk, gab, ida, lan, osp, l-o, mug, arro, mil, bil), bus (hau, ath), buus (men), buuz (etx, l-z), buz (bard, ilh, l-b, urk)

buruzagi (25): bourouzaguiac (lekb), buüssaguiac (o-z), buçaguiac (g-z), buçaguias (hau), buraçagik (men), buruçaguiac (ainh, be-l, soh, laka, larr, müs, ath, z-p), buruçaguiaz (g-i), buruçaguic (arro), buruçaguik (bil), burussaguiac (lig, irur), buruzagiak (gar), buruzagic (esk), buruzagik (bar), buruzagiz (ürr), buruzaguiaz (alo), buuçaguiaz (etx, $1-z)$

dailatu (6): daillaturik (alo), daillatuic (etx, l-z), daillaturic (alt, g-i, z-p)

dei (1): dei (aiz)

deitatu (11): deïtatceco (aha), Deïtatzen (jats), deïthatzeco (luk), Deïthatzen (luk), deitatceco (larz), deitezen (ürr), deithacen (lan), deithatcen (arm, arm), deithatcera (d-i), deythatceko (ora)

deitoramen (1): deithoamenac (urd)

deitu (194): déïtcéco (hen), Déitcén (ain), Déitcénn (hen), déitceco (ain), déitceko (don), déicent (alt), déitceco (ath), déitcen (ath), Déitcen (etx), déitcera (alt), déithouco (kan), déitséco (kan), deïtcéco (dona), deïtcea (altz), deïtceco (arb, l-z, s-g, sen), deïtceko (hau), deïtcen (altz, altz, altz, hau, s-g), deîtcen (sen), Deittcen (l-z, sen), deïthuak (gar), deïthuko (altz), deitzeco (lekb, meh), deitzeko (alo, buz, gam, gar), deitzen (ahe, lek, gar, gar, haz, haz), Deïtzen (alo, meh), deicen (alt), deitçen (lig), deitcën (g-z, g-z), deitcea (osp), deitceco (ainh, amo, arü, be-l, bir, eih, soh, sarr, ünd, etx, etxa, g-i, get, ida, its, mak, mau, mend, müs, urd, ort, zal, u-g, ure, urr, uzt), deitceko (arr, esp, laka, men), deitcen (a-m, arb, arn, a-zo, arr, altz, altz, beho, be-l, eih, g-zo, soh, ida, izt, jut, laka, las, mar, leko, mug, mug, müs, müs, ora, o-z, o-z, ort, zal, ure, urr), Deitcen (ainh, amo, beho, g-i, its, mak, mau, men, mend, mit, urd, o-z, o-z, dona), Deitcendiat (esp), deitcera (hal, jat, larr), Deithcen (osp), deithu (aih, bida), Deithuko (bida), deithzen (lekb, lekb), deitseco (o-z), deitsen (o-z, o-z), deitzéco (lah), deitzeco (bid, esk, iru, lak, l-a, sar, dono, bai), deitzeko (ürr, azka, bar, zib, l-o, mit), deitzen (aiz, ame, arü, aih, aih, bar, bar, bas, be-l, lek, sarr, d-b, d-b, ünd, gam, garr, hal, jat, lan, lan, l-a, sar, sar, bai, urk, ur-p), Deitzen (ahe, bid, bir, esk, gam, iru, l-o, dono), deitzerat (zib), deizeko (aiz), deytceco (pag), deytceko (bil), deytcen (bil), Deytcen (bil), Deytcendiat (pag)

dena (201): déna (ain, ain, ainh, a-z, a-z, beh, bit, kan, etx, hen, hen, iba, ito, ito, lah, l-s, l-s, z-p), dénac (ain, lah), dena (aiz, zar, zar, ahe, ainh, alt, alo, alo, ame, amo, amo, anh, anh, anh, arb, arb, arb, a-zo, arr, arr, arr, arh, arü, ürr, a-s, azk, azka, aih, beh, b-l, ban, 
bar, bard, bard, bas, bid, bida, bir, bir, bir, bes, d-s, d-s, buz, buz, kan, g-z, g-zo, sarr, zib, zib, d-b, d-b, ünd, esp, esk, ezt, ezt, etxa, gab, gam, gar, gar, garr, g-i, get, hel, hal, ida, iho, iho, ilh, ilh, iri, izp, izp, izt, izt, jat, jats, l-b, l-b, lak, lak, laka, laka, lekb, lekb, lah, lan, larz, larr, lar, las, las, ald, osp, l-z, l-a, l-o, l-o, luh, luk, luk, mak, mak, mak, mar, mau, leko, leko, leko, men, mend, mend, mit, mug, mug, müs, ora, ora, urd, o-z, izu, pag, arro, d-l, d-l, d-g, d-i, d-i, sen, don, hir, hir, sar, zal, zal, zur, zur, dono, bai, dona, dom, suh, suh, u-g, u-h, urk, ure, ure, ur-p, ur-p, urr, uzt, mil, bil, bil), denac (anh, arh, ban, bes, get, hel, hen, lar, las, ald, mak, d-i, sen, dom, u-h, ure, ur-p), denak (ahe, bard, zib, leko)

denbora (27): ber_denboran (bar), Ber_denboran (altz), Démbora (ur-p), demboan (altz), dembora (garr, luh, luh), Dembora (d-s, las, l-o, izu, sen), demboran (izt, osp), Demboran (iri), demboras (esp), demboraz (meh), (demboraz) (bida), demboretan (sen, hir, sar), denboan (altz), denbora (zib, d-l), denboran (zib), denboraz (ahe), denboretan (luh)

denetarik (2): dénétac (ur-p), Denetarik (ahe)

desgisatu (1): desguisatcen (get)

deus (257): déüz (beh, irur), déüze (ath), déüzere (ath), déouçic (hen), déoujéré (ito, ost), déous (aint, zar), déousé (don), déousérè (bit), déouséré (bit), déousic (aint, kan, kan, lah), déousjé (iba), déousjéré (ost), déoussic (ain, ain, lah), déouz (bun), déouzere (bun), déus (beh), déusé (l-s, l-s), déusic (alt), déuz (lig, irur), deüjic (d-g), deüs (ainh, alo, arr, arü, d-b, ünd, etx, gab, l-z, luk, luk, s-g, bai, ure, ure), deüsé (a-z, g-z, g-z), deüse (alo, a-z, lekb), deüsere (ainh, lekb, pag, s-g), deüsic (iru), deüsik (arb, arr, d-b), deüsse (gab), deüzé (hau, hau), deüzéré (etxa), deüze (arh), deüzerik (aiz), deüzic (arh), deouchic (sen, sen), deouje (aha, iba), deous (jut, jut), deousere (buz, buz), deousic (dona), deousik (a-s), deouz (a-s), deujik (d-g), deujs (ban, ban), deus (ame, anh, arb, azk, azka, aih, bar, bar, bas, beho, be-l, bid, bes, eih, soh, sarr, sarr, ünd, ezt, ezt, gar, hal, iho, iho, iri, iri, izp, izt, its, its, jat, jats, lan, osp, mau, mug, ora, ora, hir, hir, sar, dono, dom, suh, suh, u-g, urr), Deus (d-s), deusée (o-z), deuséré (o-z, z-p, z-p), deuse (a-m, ürr, jats, mend, mit, izu, izu), deusee (amo, men), deuser (bid), deusere (a-m, amo, arm, arn, arü, beho, be-l, soh, zib, esk, esk, g-i, g-i, hoz, ida, laka, laka, las, las, l-a, l-a, mit, müs, müs, arro, arro, zal, zal, urr, bil), Deusere (lar), deuseren (ida), deuserre (gar), deusic (ahe, azk, b-l, b-l, bir, bir, bes, eih, get, izt, lak, lak, mar, mug, ort, ort, d-i, d-i, dono, bai, dom, u-g, u-h, u-h, ur-p, ur-p, uzt), deusik (ahe, bas, bida, zib, esp, esp, l-o, l-o, d-l, sar, zur, urk, urk, mil, mil), deusse (anh), deussic (lan, mak, mak), deuz (ame, larr, larr), deuzé (altz, altz, mau), deuzéré (lig), deuzein (azka), deuzere (ürr, etxa, urd, bil), deuzik (garr), jeus (d-s), jose (ilh), uesse (mend), yéoussic (zar), yéouzé (gam), yeous (hel, hel), yeousé (gam), yeousik (bard), yeus (g-zo, larz, larz), yeusic (meh), yeusik (leko, leko), yoose (l-b), yoouséré (a-zo), yosé (l-b), yose (ilh), yeauje (aha)

dirdira (1): dirdira (d-s)

dirdirant (9): dirdiran (aih, arn, kan, haz, izt, uzt), dirdirant (ban, lek), dirdirantic (dom) dirdiratu (3): dirdiâtzen (zur), dirdiratcen (a-z), dirdiretan (bard)

distiradura (2): dichtiradura (zib), dichtiraduraz (bida)

distirant (39): dichtillanta (sen), dichtiran (mil), dichtirant (d-b), dichtiranta (d-b), distian (bes), distiant (etx, l-z, ure), distirant (ahe, anh, arb, arr, arm, bas, bid, buz, gam, 
hel, iri, iru, lak, d-l, hir, sar, dono, dona, suh), distiranta (bir, hel, hen, leko, ora, d-i), distirante (meh), distirantic (get, bai), distirantik (esp), distirantirik (ur-p), diztiran (jats)

distirantasun (1): distirantasun (mug)

distiratu (18): dichtiratzen (lah), disdiratcen (azk), distiatzen (izp), distiratcen (b-l, lar), distiratourik (a-s), distiratsen (luh), distiratua (its, ort), distiraturic (s-g), distiratzeco (ame), distiratzen (hal, jat, lan), distiretan (iho, mak), listreatcen (be-l, soh)

dohain (1): dohain (zal)

dohakabe (10): dohacabeac (uzt), Dohacabeac (iri, hir), dohacabiac (aint, zar, zal), (dohacabiac)

(luk), dohakabeak (sar), dohakabek (gar), dohatkabiak (l-o)

dohatsu (6): dohatsou (ain), dohatsu (uzt), dohaxu (gar, lar, hir), dohaxuak (sar)

dolamen (3): dolamenac (arn, azk), dolhaménac (ain)

Done (273): dene (ilh), Dene (l-b), Don (lekb), Dona (bard), done (hal, iri), doni (ahe, ahe, iri, jut, lar, meh, meh), Doni (d-s, jut, ost, ost, ost), Tjondoni (hen), Tjonndoni (hen), Uundane (urk), Yaündoni (ure), Yandani (buz), Yandoné (zar, zar, azka), yandone (iba), Yandone (arm, arm, azka), yandoni (gam), Yandoni (buz), Yaundoni (ure), yoandané (bit), Yoandané (bit), yoandene (garr, garr), yoantcen (izt), yodoni (dom, dom), yon (ahe, ahe, iri), Yon (hal, iri, meh, meh, ost, ost, ost), Yonboni (larz), Yondane (arh, l-o, l-o, pag, pag, u-h, u-h), youandéné (beh, bun), Youandéné (beh, bun), Youandene (a-zo), (Youandene (a-zo), Youandoni (a-z, a-z), Youndane (ünd), Yuandoni (luh), Yundane (urk), zan (a-s), Yondoni_(ain), yondoni_ (bes, esp, bai, dona), Yondoni_ (ame, anh, arb, arr, azk, b-l, bid, bida, gab, get, hel, iba, iru, lan, luh, mend, d-l, d-i, sen, sar, suh, u-g, urr, uzt), Yondoni- (ur-p), Yondoni-" (ur-p), Yondo_ (jats), Joundan (g-i), Joundané (hau, hau, lig, lig, mau, mau, o-z, o-z, ath), joundane (arü, arü, sarr, sarr), Joundane (ainh, alo, alo, ürr, ürr, bar, bar, soh, soh, ünd, esk, esk, etx, etx, etxa, etxa, g-i, ida, ida, laka, laka, lekb, lekb, larr, larr, l-z, l-z, l-a, l-a, mit, urd, urd, arro, arro, zal, zal, ath, irur, irur), Joundane- (l-z), -Yondoni (mend), Joundaen- (ida), Joundane- (etx), Yondane- (pag), Yondone (hal, ald, izu), yondoni (aiz, bida, esp, gam, haz, izt, leko, leko, mug, sar, bai, dona, u-g), Yondoni (ain, ame, anh, arb, arr, azk, b-l, bid, lek, lek, bes, g-zo, g-zo, gab, gab, get, hel, haz, iru, iru, jat, jat, jats, lah, lah, lan, larz, las, las, ald, ald, luk, luk, mak, mak, mug, mug, izu, sen, don, don, zur, zur, dono, suh, urr, uzt), Yondoni- (zur), jondane (men), Jondane (men, müs, müs), Jondone (s-g, s-g), jondoni (d-l), Jondoni (d-i, hir, hir), Jondana (arh), jondon (ban), jondone (ban), Jondoni (bas, bas), Jondane (be-l, be-l), Jondoni (bir, bir), Jondoné (kan, kan), Jondoni (zib, ezt, ezt, ezt), Jondane (gar, gar, alt, alt), Jondoni (hoz, hoz, iho, izp, izp), Joan-déné (ito), Jondoni (its, its), jondoni (lak), Jondoni (lak), joandoni (lar), Jondane (osp, osp), Jüandeni (d-b), Juandeni (d-b), Jundane (mit), Jaoun-Doni- (ora), JaounDoni- (ora), jon doni (lar), Jon Joundane (ürr), Jon Doni (d-s)

dorpe (1): dorphe (zib)

duda (4): doudaric (bun), dudaic (osp), dudaric (lar), dudarik (lek)

ebaki (97): ébakia (beh, bun, gam, ost), ébakiac (ain, a-z), ébakiak (beh), ébakiic (g-z, etx), ébakiik (ath), ébakirik (ünd, lig, irur), ébaquiac (a-zo, bit, iru), ébaquic (l-s, o-z), eakiac (hel), eakiak (bas, leko), ebakhiac (arn), ebakhik (men), ebaki (izp, dona), ebakia (aha, a-m, arh, bard, bid, buz, gab, hoz, jut, lak, larz, luk, mak, mug, ora, ort, d-g, dom, u-h), ebakiac (ame, anh, aih, b-l, iba, lan), ebakiak (arm, a-s, d-s, zib, d-b, 
garr, jats, l-o), ebakidienian (pag), ebakiic (osp, l-z, izu), ebakik (altz), ebakina (mil), ebakiric (ainh, arü, ürr, be-l, esk, g-i, ida, laka, lar, l-a, mit, müs, urd, arro, s-g, zal, suh), ebakirik (bar, sarr, gar), ebaquia (amo, bai, eih), ebaquiac (g-zo, hal, izt, jat, mar), ebaquiric (soh, etxa, mau), ebaquitiac (d-i)

ebasle (1): ebaxletaric (urd)

ebatsi (146): ébach (ito), ebachtetic (sen), ebasteco (urr), ébasteko (azka), ebasterat (uzt), ebasteti (altz), ebastetic (l-z), ébastetic (etx, lig, irur), ebastetik (ahe), ébats (ain, zar, alt, a-zo, bun, kan, gam, ilh, iru, l-b, lig, l-s, don, irur), ebatsak (urk), ebatse (meh), ebatsi (lan), ébatss (bit), ébatx (a-z, hau), ebatxic (osp), ébatz (beh, hen), ébax (g-z, lah, z-p), ébazt (ur-p), eatj (iba), eats (bir, hel), eatz (mend), ebatch (aha, sen), ebatjs (ban), ebats (ahe, alo, arm, arn, bar, bas, beho, bid, bida, lek, bes, buz, zib, d-b, esp, esk, ezt, etxa, gab, hal, haz, iho, iri, izp, izt, its, jat, jats, jut, larr, las, luk, leko, men, mug, ora, o-z, ort, s-g, d-l, d-g, hir, zal, zur, dono, bai, suh, ath, u-g, mil), ebatx (ainh, arb, arr, arü, b-l, eih, soh, sarr, ünd, laka, lekb, mak, mit, ost, dona, dom), ebatz (aiz, ame, arh, a-s, azk, bard, garr, get, hoz, ald, l-o, d-i, u-h, bil), ebax (amo, anh, ürr, aih, be-l, g-zo, gar, g-i, lak, larz, l-a, mar, mau, müs, izu, pag, arro, sar, ure)

edan (145): édéaten (garr), édan (kan), édatén (ain, iba, ito, don), édatènn (bit), édaten (alt, l-s, mug, o-z), édatenn (hen), édates (ath), édatez (etx), édatiaïkin (a-z), édatiaékin (beh), édatiaikine (ost), édatiaeki (hau), édatian (aint, bun, zar), édatiéqui (g-z), édatiarequi (z-p), edanes (lar), edanez (bas, bes, zib, iho, izt, jats, mak, meh, mend, ora, d-l, dona), edatearequin (ame), edaten (aha, ahe, ainh, amo, arb, a-zo, arr, arm, arü, azk, azka, altz, beho, b-l, ban, bid, bida, d-s, buz, eih, g-zo, sarr, d-b, ünd, esp, ezt, etxa, gam, g-i, hel, hal, hoz, ida, iri, iru, izp, its, jat, laka, lig, lan, larr, las, ald, osp, l-a, l-o, mar, mau, leko, müs, urd, ort, pag, s-g, d-g, d-i, sen, hir, sar, zur, bai, dom, suh, irur, u-g, ure, urr, uzt, mil, bil), edatez (bar, be-l, soh, esk, lekb, l-z, arro), edatiaakin (aih), edatiaeki (alo, men), edatiaikin (gab, larz, izu), edatiaiquin (jut), edatiakin (bard, ilh), edatian (anh, arh, bir, lek, get, haz, dono, u-h, urk), edatiareki (gar), edatiarekin (aiz, ürr, a-s, lak, luk, mit), edatiarequi (zal), ediatiakin (l-b), edatean (arn), edatiaïkin (a-m)

eder (1): édér (bit)

edertto (2): edertto (hal, jat)

ediren (60): édién (sarr, hau), édieïtén (hau), édieïten (etx), édieiten (o-z), édien (alt, etx, ath), édienten (ath), édireyten ( $z-p$ ), edieïten (altz, l-z), edieiten (arü, sarr, ünd, men, urd), edien (amo, ünd, l-z, mar, pag), edieyten (pag), ediréïten (irur), edireïten (alo, amo, lekb), edireiten (ainh, ainh, ürr, bar, be-l, soh, esk, etxa, gar, g-i, laka, lig, l-a, l-o, l-o, mau, mit, müs, müs, arro, s-g), ediren (alo, arü, ürr, esk, ida, lekb, arro, z-p), edireyten (larr, bil)

edo (254): édo (ain, aint, zar, zar, alt, a-z, a-z, beh, bit, bit, bun, kan, kan, etx, etx, hen, hen, iba, ito, l-s, l-s, ost, ost, don, ath), edo (aha, aint, aiz, aiz, ahe, ahe, alt, alo, alo, a-m, ame, beho, eih, ame, amo, anh, anh, arb, a-zo, a-zo, a-zo, a-zo, arr, arh, arh, arm, arü, ürr, ürr, a-s, a-s, azk, azka, altz, aih, aih, beh, b-l, b-l, b-l, ban, bar, bar, bard, bard, bas, bas, bas, be-l, bid, bid, bida, bir, bir, lek, bes, bes, bun, d-s, buz, buz, g-z, g-z, g-zo, g-zo, soh, sarr, zib, d-b, d-b, ünd, esp, esp, esk, esk, ezt, ezt, etxa, etxa, gab, gab, gam, gar, gar, g-i, g-i, get, get, hel, hal, hal, haz, hau, hau, hoz, hoz, iba, ida, iho, ilh, ilh, iri, iru, izp, izp, izt, izt, its, its, jat, jat, jats, jats, jut, jut, l-b, l-b, lak, lak, laka, laka, lekb, lekb, lig, lig, lah, lan, lan, larz, larz, larr, lar, las, ald, ald, osp, l-z, l-z, l-a, l-a, l-o, 
l-o, luh, luk, luk, mak, mak, mak, mar, mar, mau, mau, meh, leko, men, men, mend, mend, mit, mit, mug, mug, müs, müs, ora, ora, urd, o-z, o-z, ort, ort, izu, izu, pag, pag, arro, arro, s-g, s-g, d-l, d-l, d-g, d-g, d-i, d-i, sen, sen, hir, sar, zal, zal, zal, zur, bai, bai, dona, dom, suh, irur, irur, u-g, u-g, u-h, u-h, urk, urk, ure, ure, ur-p, urr, uzt, mil, mil, bil, bil, bil, z-p), (edo (arü, arü, g-zo, g-zo, g-zo, meh, meh, pag), Edo (a-zo)

egarri (16): égari (kan, o-z), égarri (iba, lah, mit), egari (mau), egarri (aiz, anh, ürr, a-s, bar, bard, bida, etxa, arro), egarria (mar)

egiazki (6): éguiazki (ain), egiazki (zib, d-l), eguiaski (jat), eguiazki (ezt, hal)

egile (2): eguile (anh), eguilen (altz)

egin (449): éitia (ito), égin (ito), éginén (ito), égitén (ito), éguin (ain, ain, a-z, bun, kan, lig, lig, l-s, urd, don, ath, ath, ur-p, z-p), éguina (hen, lah), éguinènn (bit), éguincéçac (z-p), éguinic (alt, etx), éguinn (hau), éguissac (bun), éguitèco (bun), éguitéco (a-z, ost, z-p), éguitén (hau, ost, don, don), éguiteco (arm, lig, mug, dom, ur-p), éguiten (a-z, a-z, a-s, kan, hau, lig, lah, lah, l-o, mend, z-p, z-p, z-p), éguitencuen (ur-p), îten (zur), e'iten (ban), eïn (gab, gab, hel), eina (haz), eiten (bard), egik (buz, l-o, sar), egin (ürr, ürr, bida, d-s, buz, buz, sarr, zib, zib, ünd, esk, hoz, l-b, meh, meh, men, mit, mit, d-l, d-g, sar, zur), egina (arn, lak, u-g, u-h, mil), Egina (lek), egindadiela (hoz), egineçak (men), eginik (bar, l-o), egitéco (d-g), egiteco (beho, meh, u-h), egiteko (haz, zur), egiten (arn, bar, d-s, d-s, zib, zib, haz, hoz, hoz, iho, lak, men, d-l, d-g, d-g, sar, sar, u-g, u-g, u-g, u-h, mil), egitendu (d-g), egiterat (ald), egiuten (sen), eguic (ida, lekb, dom), eguin (aiz, aiz, ahe, ainh, alo, a-m, ame, amo, anh, arb, arb, arr, arr, arh, arm, arü, azk, ban, be-l, bida, eih, g-z, soh, esp, ezt, ezt, etxa, g-i, get, ida, ilh, iri, iru, izp, izt, jat, jats, lak, laka, lan, lar, las, ald, mau, mug, müs, ora, pag, arro, arro, s-g, s-g, d-i, d-i, sen, hir, dono, dono, dona, irur, irur, ure, uzt, bil, bil, bil), Eguin (hal, jat, lar), eguina (aiz, arh, beho, b-l, iho, its, lekb, luh, luk, mak, mend, ort, d-g, dom, suh, urr), eguinçac (eih, ure), eguinçan (ure), eguinac (lar), eguinan (lekb), eguinen (ame, ezt, lan, ald, luh, suh, uzt, mil), eguinezac (jats), eguinezan (jats), eguinic (larr, l-z, l-a, o-z, zal), eguinik (a-s, altz, gar), eguinzak (aiz), eguisan (luh), eguitéco (g-z), eguitén (hau), eguitea (aiz), eguiteco (a-m, ame, arh, a-s, azk, ban, lek, hoz, izp, jats, las, mar, arro, suh, irur), eguiten (ahe, alo, alo, ame, amo, anh, anh, anh, arb, arr, arh, arm, a-s, a-s, azk, altz, aih, ban, bida, eih, lek, lek, lek, esp, ezt, g-i, get, get, get, hal, haz, iri, iri, izp, izp, izt, its, jat, laka, lan, larz, larz, lar, lar, las, ald, ald, ald, l-a, luh, luh, luk, mau, mau, meh, meh, men, mend, müs, ora, ora, urd, pag, arro, arro, d-i, d-i, sen, sen, hir, hir, dono, dom, dom, dom, dom, suh, ath, mil), eguiteti (altz), eguitin (izp), eguizu (ezt), eiçak (esp), ein (bard, bas, bid, bir, bai), eina (leko), eindaïla (urk), eintsak (bard), eisak (leko), eitea (bes), eiteko (bard, urk), eiten (bard, bid, bir, bir, bes, bes, bes, leko, bai, urk), Eizak (urk), eyteco (d-s), eyteko (bas), hin (beh), in (a-m, aint, zar, a-zo, aih, g-zo, gab, jut, jut, izu), ina (aha, azka, aih, bit, larz, ost), indailla (gam), inen (a-zo, g-zo, g-zo), inendinat (a-zo), inic (a-zo, g-zo), Intzac (haz), isac (l-s, ald), itéco (aint, zar, l-s), itéko (beh), itén (ito, l-s, ost, ost), iteco (a-zo, g-zo, gab, iho, jut, izu), iteko (bit, ilh), iten (aha, aint, aiz, zar, a-zo, a-zo, azka, beh, g-zo, g-zo, d-b, d-b, gab, gab, gam, gam, garr, garr, garr, iba, iho, ilh, ilh, ilh, jut, jut, l-b, l-b, larz, l-s, izu, izu, izu, ur-p), itendiat (zur), izac (a-m, meh)

egoitza (2): égoitça (bida), Egoitça (bida)

egon (7): égoïten (alt), egoïten (beh), egoitea (iru), egoiten (l-a, arro), egonasten (izu), 
egoneazten (laka)

eguerdi ondo (22): eguerdi_ondo (a-m, arn, beho, mend), eguberdi onduan (urr), egouerdi ondo (arm, a-s, bard), egouerdi ondoatez (beh), égouérdi (bit), egordi oundo (ilh), égouardi ondo (ito), eouguerdi ondo (jut), egardi oundo(l-b), eguerdi_ondo (alo, izt, jats, larr, las, meh, mug, dono, suh, u-g), Eguerdi_ondo (zar), Eguerdi ondo (aint)

egun (465): aïgun (ito), égoun (bun, iba, lah, don, don), Égoun (ain), égounéan (ain, ain), égounéco (bun), égounco (ain, ain), égoune (ost), égoungo (bit, bit, lah, ost), égounian (bit, bun, lah), égouniane (ost), égouniann (bit), égun (a-z, beh, ünd, etx, hau, hau, lig, lan, l-s, o-z), Eün (d-b, leko), eünean (leko), eüngo (leko), egün (g-z, g-z, gar, gar), Egün (g-z, d-b), egoun (aha, arh, arm, a-s, a-s, eih, ilh, jut, jut, sen, dona), Egoun (aha, arm, a-s, bard, bit, bun, buz, buz, kan, gam, hen, iba, iba, jut, lekb, lekb, lah, luh, ora, d-g, sen, dona), egounco (ora, ora), egounean (luh, sen), egoungo (bard, dona), Egoungo (bard, sen), egounian (buz, ora, dona), egounien (bard, bard), Egumbates (lar), egun (alo, alo, ame, amo, amo, anh, arn, a-zo, arh, arü, beho, ürr, ürr, azk, azka, azka, altz, bar, be-l, bid, bir, lek, g-zo, soh, sarr, esk, etxa, etxa, gab, gab, gam, g-i, haz, ida, ida, iho, ilh, iri, iru, izp, izt, its, its, jats, jats, l-b, l-b, lak, lak, laka, laka, lekb, lan, larz, las, ald, ald, l-z, l-a, l-o, mak, mak, mar, mau, mau, meh, men, men, mend, mend, mit, mit, müs, müs, urd, ort, ort, s-g, d-l, d-g, d-g, zal, zal, dono, dono, bai, dom, suh, ath, u-g, u-g, u-h, u-h, ure, urr, uzt, uzt, mil, mil, bil, bil, z-p), Egun (aiz, ahe, ainh, ainh, aint, alt, alo, a-m, ame, ame, amo, anh, a-z, a-z, arb, a-zo, arr, arh, arü, arü, beho, eih, ürr, azk, azka, altz, altz, beh, b-l, ban, bar, bar, bas, be-l, be-l, bid, bida, lek, bes, d-s, kan, g-zo, soh, soh, sarr, sarr, zib, ünd, ünd, esp, esk, esk, ezt, etx, etx, etxa, gab, gam, gar, garr, g-i, g-i, get, hal, haz, hau, hoz, ida, iho, ilh, iri, iru, izp, izp, izt, ito, its, jat, jats, l-b, lak, laka, lig, lan, larz, larz, larr, l-s, las, las, ald, osp, l-z, l-z, l-a, l-o, l-o, luk, mak, mar, mar, mau, meh, men, mend, mit, mug, müs, urd, ost, o-z, ort, izu, izu, arro, s-g, d-i, don, hir, sar, zal, zur, dono, bai, bai, dom, suh, suh, ath, ath, irur, irur, u-g, u-h, ure, ure, ur-p, urr, urr, uzt, mil, bil, z-p), Egunbatez (pag), egunco (altz, arro, arro), egunco_egunian (larr, osp, osp), Egunco_egunian (s-g), egunean (b-l, b-l, bida, bida, zib, zib, get, iri, iru, mug, mug, d-l, d-l, d-i, d-i, hir, sar, sar), egunetan (aiz, lek, garr, haz, d-l), egungo (anh, arb, arr, b-l, ban, bida, bida, bes, buz, esp, ezt, garr, get, get, hoz, izt, luk, luk, meh, mug, d-l, d-i, d-i, hir, sar, sar, zur, zur), Egungo (aiz, arb, a-zo, arr, b-l, ban, bes, g-zo, esp, hoz, iri, l-a, mug), egunian (anh, arb, arb, a-zo, arr, arr, azk, ban, ban, bas, bes, g-zo, esp, esp, ezt, get, hal, hal, hoz, hoz, izt, jat, jat, l-a, luk, luk, meh, ora, zur, zur), egunin (altz, bes, arro, arro), eoun (hel, hel), egunn (am), Eoun (zar, hel), eun (urk, urk), Eun (aih, aih, bid, bir, urk), eunean (leko), Eungo (leko), eygun (pag, pag, z-p)

egur (3): égourrénn (hen), égourrétànn (hen), égourrétic (hen)

ehelegatu (1): ehelegatu (arro)

eia (1): eya (lar)

eijer (48): éijer (lig), éjer (altz, hau, ath), eïger (zal), eïher (ünd), eijer (gar), eder (ahe, arb, arr, aih, bun, zib, esp, d-l, sar, urk, urr), eger (ainh, pag), eiger (soh), eijer (etx, l-z, l-a, o-z, s-g, irur), ejer (arü, ürr, bar, be-l, sarr, esk, g-i, ida, laka, larr, osp, mau, men, mit, müs, arro, z-p), ejerbatétan (alt), ejerbatetan (alo, urd), eyjer (bil)

eitzi (1): éisi (alt)

ekarri (13): ekarazten (anh), Ekarrazten (aih), ekhararasten (b-l), Ekharraaazten (hel), 
Ekharraassico (bun), ekharrarazteco (arn), ekharrarazteko (ezt, mil), Ekharrazico (bas), ekharrazteco (hir), ekharrazten (aha, buz), ekharri (ban)

ekuratu (27): ekhuatu (altz, g-i, larr, l-a), ekhuazten (alo), ekhuratu (s-g), ekhuratubat (g-i), ukhuatu (ainh, alt, be-l, etx, hau, ida, lig, lig, osp, l-z, mau, o-z, ath, irur), ukhuratu (alo, bar, soh, esk, arro, irur)

ele (1): Elhe (altz)

elge (19): élgiac (ito), elgiak (d-b, l-b), elgueac (ame), elguiac (aiz, amo, a-z, arü, etxa, luk, ora), Elguiac (a-zo, g-zo, gab), elguiak (beh, bard, garr, ilh)

eli (39): bestélibatetan (o-z), bestelibati (gar), élibat (alt, altz), élibatec (altz), élibatez (altz), eli (ainh, g-z, g-z, g-z, etxa, larr, mau, müs), elibat (g-i, laka, müs, arro, zal, irur), elibatec (esk, urd), elibatetan (be-l, soh, osp, urd, bil), elibatez (ürr, bar, bar, be-l, soh, esk, g-i, ida, osp, mit, bil, bil)

elizate (3): élissa athila (l-s), eliçatherat (arh), elizatherat (u-h)

elkartu (1): elkhartino (bil)

elki (2): elkhi (ainh), ilkhi (d-l)

elur (148): élhourès (kan), élhouréz (ain), élhourrés (ost), élhourréz (bit), élhourrez (bun, don, ath), élhurés (1-s), élhurez (etx), élhurrés (ito), élhurrez (beh), élurrez (ur-p), elhürrez (d-b), elhoures (luh), elhourez (arh), elhourraz (lekb), elhourrèz (hen), elhourrez (aha, aint, eih, zar, arm, a-s, buz, gam, hel, jut, lah, ald, mend, ora, sen, dona), elhurés (g-z, hau), elhures (o-z), elhurez (alo, ünd, ida, lak, lig, l-z, l-o, mar, mau, meh, müs, zal, dom, irur, bil, z-p), elhurra (alt, b-l), elhurraz (ort), elhurres (arb, arr, ban, esp, lar), elhurretan (iho), elhurrez (aiz, ahe, ainh, a-m, amo, anh, arn, a-z, a-zo, arü, beho, ürr, azk, azka, altz, aih, bar, bard, bas, be-l, bida, lek, bes, d-s, g-zo, soh, sarr, zib, esk, ezt, etxa, gab, gar, garr, g-i, get, hal, haz, hoz, iba, ilh, iri, iru, izp, izt, its, jat, jats, l-b, laka, lan, larz, larr, las, osp, l-a, luk, mak, leko, men, mit, mug, urd, izu, pag, arro, s-g, d-l, d-g, d-i, hir, sar, zur, dono, bai, suh, u-g, u-h, urk, ure, urr, uzt, mil), elurrez (bid, bir), helhurraz (ame), elhourrez (), elhurrez ()

eman (340): Casumac (bid), emoc (azk), Gogoemac (bit), Gogouman (irur), Gomac (az), gomac (a-zo), gomak (garr), Goman (ort), gomaneçan (a-zo), Gooemac (g-zo), gooemaneçan) (g-zo), Gouardémac (iba), Gouardiemak (l-b), Gouardimac (ost, dona, azka), Gouardimak (gam), Guardaman (lan), Guardiemac (uzt), Guardi'emac (ban), (Guardi'eman) (ban), Guardimac (d-g, dono, u-g, larz), émaïtènn (bit), émaïten (don), émak (beh), éman (ain, ain, ain, aint, zar, alt, a-z, a-z, bun, kan, kan, kan, kan, etx, etx, ito, ito, lig, lah, lah, l-s, l-s, ost, o-z, o-z, irur), émann (bit, bit, hau, hen, ost), emaiten (lekb), Emaçue (its), emac (ahe, ame, anh, arb, arh, aih, bas, beho, be-l, bir, soh, get, iho, iho, iru, izp, izt, lak, lar, las, luk, mak, ora, d-i, suh, u-h, ur-p, urr), emaiten (iri, izt, lan, lar, lar, ald, l-o, luh, dom, suh, uzt), emak (a-s, bida, ilh, jats, mil), Emak (d-l), eman (aha, ahe, ahe, ainh, aint, aiz, alt, alo, a-m, arn, beho, eih, zar, ame, amo, anh, anh, arb, arb, a-zo, arr, arr, arr, arr, arh, arh, arh, arm, arm, arü, arü, ürr, a-s, azk, azk, azka, altz, altz, aih, aih, beh, b-l, b-l, ban, bar, bar, bard, bard, bas, be-l, be-l, bid, bid, bida, bir, bir, lek, lek, bes, bes, bes, d-s, d-s, buz, g-z, g-zo, soh, soh, sarr, zib, zib, d-b, d-b, ünd, esp, esp, esk, ezt, ezt, etxa, gab, gam, gar, gar, garr, garr, g-i, g-i, hel, hel, hal, hal, haz, haz, hoz, hoz, hoz, iba, ida, iho, iho, ilh, iri, iru, izp, izp, izt, izt, its, its, jat, jat, jats, jut, l-b, lak, lak, lak, lak, laka, lekb, lan, larz, larz, larr, lar, las, ald, osp, osp, l-z, l-z, l-a, l-a, l-o, luh, luk, mak, mak, mar, mau, meh, meh, leko, leko, men, mend, mit, 
mug, mug, müs, ora, ora, urd, ort, ort, izu, pag, pag, arro, arro, s-g, d-l, d-l, d-g, d-i, sen, sen, hir, hir, sar, sar, sar, zal, zal, zur, dono, dono, bai, bai, dona, dona, dom, suh, suh, suh, ath, u-g, u-h, u-h, u-h, urk, urk, ure, ure, urr, urr, urr, uzt, uzt, uzt, uzt, mil, mil, bil, z-p), (eman) (bida, luk), emancen (get), emancioten (hir), emann (ora, ost), emannay (ur-p), emansioten (ur-p), emantcen (esp, hir), emantxen (dono), emantzen (arb, haz, larz, dom, urk), emanzen (ur-p), ematen (zib, d-l), emoc (azk), hémann (hen), hemac (hen), man (hel)

emazte (404): émasté (a-zo, beh, beh, bit, bit, bun, hau, ito, ost, ost, ath), émastéat (ito), émaste (alt, alt, l-s, l-s, o-z), émastebat (etx), émastec (ito), émastia (alt, a-z, beh, bit, bun, kan, hau, ost, o-z), émazté (a-z, bun, iba, iba, don, don), émaztéa (ain), émazte (aint, zar, a-z, etx), émaztia (aint, zar, etx, iba, lah, don), emasté (altz, altz, altz, altz, g-z, g-z, hau), emaste (aiz, ainh, ainh, ame, amo, amo, arb, arb, arr, arh, ürr, azk, altz, b-l, ban, ban, bar, bas, be-l, d-s, und, esp, etxa, gab, get, get, hoz, ilh, jat, jat, larr, larr, lar, lar, lar, ald, ald, l-a, l-a, mak, mak, mar, mau, mau, mend, o-z, pag, d-g, d-g, d-i, d-i, dono, suh, u-h, u-h, u-h), emastea (ame, azk, get, mil), emasteat (a-zo, mend), emastebat (arü, ilh, mar, pag), emastia (ainh, amo, arb, arh, arü, eih, ürr, a-s, altz, ban, g-z, sarr, esp, etxa, hoz, ilh, larr, lar, l-s, ald, l-a, mak, mau, mend, pag, d-g, zur, dono, ath, u-h), emastiac (arr, d-i), emazt (osp), emazté (zar, izt, l-b, z-p), emazte (aha, alo, a-m, ame, ame, anh, anh, arn, arr, arh, arh, arm, arm, ürr, a-s, azka, azka, aih, aih, aih, beho, b-l, b-l, b-l, bar, bard, be-l, bid, bid, bida, bida, bida, bir, bes, bes, d-s, buz, buz, g-zo, soh, soh, sarr, sarr, d-b, d-b, ünd, esk, esk, ezt, ezt, etxa, gab, gam, gam, gar, gar, garr, garr, garr, g-i, g-i, hel, hal, hal, hoz, ida, ida, iho, iri, iri, iru, izp, izp, izt, its, its, jats, jats, jut, jut, lak, lak, laka, laka, lekb, lekb, lig, lig, lan, lan, larz, larz, las, las, osp, l-z, l-z, l-o, l-o, luk, luk, meh, meh, leko, leko, men, men, mit, mit, müs, müs, müs, ora, ora, urd, izu, izu, arro, arro, arro, s-g, s-g, sar, zal, zal, dono, bai, bai, dona, dona, dom, dom, suh, irur, irur, u-g, u-g, urk, ure, ure, urr, mil, bil, bil, z-p), emaztea (anh, arn, bida, lek, zib, hal, haz, iri, iru, izt, its, jat, leko, mug, hir, sar, ure, urr, uzt), emazteak (d-l), emaztearen (iru), emazteat (bard, g-zo), emaztebat (aiz, alo, l-b, urd, mil), emaztet (urk), emaztia (aha, alo, a-m, a-zo, arm, azka, aih, beho, b-l, bard, bas, be-l, bid, bes, d-s, buz, g-zo, soh, d-b, ünd, esk, ezt, gab, gam, gar, g-i, ida, iho, izp, jats, jut, l-b, lak, laka, lekb, lig, lan, larz, las, osp, l-z, l-o, luk, mar, meh, men, mit, müs, ora, urd, izu, arro, s-g, zal, bai, dona, dom, suh, irur, u-g, urk, bil, z-p), emaztiari (garr), emaztik (bar)

emazteki (45): émastéki (kan, kan, mug), émastéqui (hen, hen), émaztékibat (lah), émazteki (ain, ain, lah), emastéqui (ur-p), emasteki (azk, lek, hel, luh, luh, luh, luh), emastekibat (esp, mug), emastequi (ame, bir, eih, hir, ur-p), emazki (uzt), emazteki (ahe, ahe, a-s, bas, lek, lek, haz, haz, haz, sen, sen, sar, sar, zur, zur, uzt), emaztekia (ahe), emaztequi (hir, urr)

endurtu (1): endurtu (leko)

entzun (102): éntçoutiarékin (don), ëntsutiéqui (g-z), ençutearekin (izt), ençutiaéquin (alt), ençutiai (men), ensunic (lig), Ensunic (ito), Ensutiakin (bard), Entçoutiarekin (ora), entçun (altz), entçunez (g-zo), entçunic (b-l, etx, larr, l-z, mau, urd, s-g), Entçunic (etxa), Entçutés (ath), Entçutearekin (its), Entçuten (be-l, soh), entçutez (ainh), Entçutez (pag), Entçutiaïki (hau), Entçutiaïkin (a-z), Entçutiaékin (beh), Entçutian (gab), entçutiareki (g-i, arro), Entçutiareki (ida, zal), entçutiarekin (arü, mar, bil), 
entçutiarequi (müs), entçutiarequin (suh), Entçutiarequin (amo, d-i), Entçutiari (z-p), entçytiarekin (a-m), Entsoutia (bun), entsunic (irur), entsutiaéqui (o-z), entsutiaïquin (l-s), entzounik (arh, lekb), entzunic (iho, dom, u-h), entzunik (bar), entzutearekin (arn, iri), entzutearequin (iru), entzutez (esk), entzutiaekin (l-o), Entzutiaikilan (db), Entzutian (luk), entzutiareki (alo), Entzutiarekila (gar), entzutiarekin (ame, sarr, ünd, ezt, laka), Entzutiarekin (hoz, lak), Entzutiarequi (l-a), entzutiari (ürr, mit), Inçutiaekin (d-g), Inçutiarekin (u-g), intçounic (zar), intçounik (aint), intçoutiaïkin (ost), Intçoutiaiquin (jut), intçoutian (iba), intçoutiann (bit), intçoutiarekilan (aha), Intçoutiarékin (dona), Intçoutiarekin (arm), Intçutiaikin (izu), intçutiarekin (las), Intsutiaikin (mend), intzoutiaikin (gam), intzoutian (buz), intzunik (d-s, garr), intzurra (zib), Intzutiaikin (azka, larz), intzutiarekin (beho, izp), Intzutiarekin (jats), Inzoutiarekin (a-s), inzunik (aiz), Inzutiarekin (lan), untçunex (a-zo), Untçutiakin (ilh, l-b)

epain (4): éphéina (l-b), ephainac (hal, jat), epheina (ilh)

epaitu (2): ephaitia (aiz, u-g)

epantxatu (2): ephantchatceko (altz), ephantchatcen (altz)

erakutsi (2): eakouzten (garr), eracusten (iho)

eraman (11): éaman (irur), éréman (zar, bun, ito), éremanac (ur-p), eaman (lig), eraman (bir), ereman (aint, aiz, bai), erematen (luh)

erausi (2): eausitu (lek), ehausiz (iri)

erdi (1): erdian (lekb)

erdiragarri (2): erdiragarri (beh), erdiragarrico (ure)

erdiratu (54): érdiraastéco (ost), érdiratcéco (bit), erdiaraco (lar), erdiaraztéaïnocoan (a-z), erdiartekan (l-b), erdiartino (hau), erdiastekan (ilh), erdiatceco (anh, g-zo, g-i, mau), erdiatceko (laka, men), erdiatcen (arü, sarr, ünd), erdiatcera (ath), erdiatseco (g-z), erdiatu (altz, l-a), erdiatzeco (arh), erdiatzeko (garr), erdiatzekua (larz), erdiatzen (ürr, mit), erdiazteko (gar, müs), erdierazteko (soh), erdikatzeko (mil), erdiraazteko (db), erdirasteco (d-i), erdiratceco (be-l, eih, etxa, gab, hoz, ida, osp, arro, u-h, z-p), erdiratcecoa (its), erdiratcialano (s-g), erdiratu (bard, hir), erdiratuco (lig), erdiratzeco (bes, luk), erdiratzecua (d-s), erdiratzeko (alo, a-s, bid, buz), erdirazteco (esk)

ere (553): bagnore (jut), deouje (iba), déoujéré (ost, ito), déousé (don), déouséré (bit), deousere (buz), déousérè (bit), deousere (buz), déousjé (iba), déousjéré (ost), déouzere (bun), déouçic (hen), deuse (mit, izu), deüsé (a-z), deuse (ürr), deüsé (g-z), deuse (jats), déusé (l-s), deuse (mend, izu), deüse (a-z), deüsé (g-z), deüse (alo, lekb), déusé (l-s), deusee (amo), deusée (o-z), deusee (men), deuser (bid), deusere (müs, arro), deüsere (s-g), deusere (zal), deuséré (z-p), deusere (esk, g-i, ida, laka), deüsere (lekb), deusere (las, l-a, bil, mit, müs, amo), deuséré (o-z), deüsere (pag), deusere (arro, zal, urr), deuséré (z-p), deusere (arm, arü, be-l, soh, zib, esk), deüsere (ainh), deusere (g-i, hoz, laka, las, l-a, ), Deusere (lar), deuseren (ida), deuserre (gar), deusse (anh), deüsse (gab), deüze (arh), deüzé (hau), deuzé (altz, altz, mau), déüze (ath), deüzé (hau), deuzere (urd), déüzere (ath), deüzéré (etxa), deuzere (ürr, etxa), deuzéré (lig), deuzere (bil), deüzerik (aiz), é (altz, altz, hau, hau, hau, l-s, l-s), ê (zur), 'ê (ban), e (etx, jut, jut), étaaré (gam), etaare (gam, gam), etare (arü, garr, arü), étaré (zar), etare (ure, ure), étaré (zar), etare (ure), ihourcere (müs), ihourkere (pag, ath, ünd, ainh), Ihourkere (alo), ihourkere (alo), ihourquéréc (etxa), issaniké (bard), izanike (garr), izanikée (gam), izanikere (aiz), 
izanique (bir), izaniqueré (ur-p), jose (ilh), neho'cke'e (ban), nehokec (izu), nehokee (bid), nehokée (gam), nehokee (larz), Néhoque (iba), nihorkec (mar), nihouké (ito), nihoukec (gab), nihoukéek (beh), nihoukek (mar), nihourcek (urd), nihourkec (men), nihourqué (l-s), Noizee (mend), Nouize (men, men), nouizee (men), nuizee (men), Oraiéé (beh), orayere (ur-p), uesse (mend), yeousé (gam), yeousik (bard), yéoussic (zar), yéouzé (gam), yoose (l-b), yoouséré (a-zo), yose (ilh), yosé (l-b), éé (alt, alt, o-z), êê (ban), ée (etx, etx, etx, etx, etx), èrè (bun), éré (ain, aint, zar, ainh, ainh, ainh, alt, alt, alt, a-z, a-z, beh, beh, bit, bit, bit, bit, bit, bun, bun, bun, bun, bun, bun, kan, kan, kan, g-z, etxa, izt, izt, izt, ito, ito, lig, lig, lig, lig, lig, lig, lah, mau, mau, mug, don, irur, irur, irur, ur-p), ére (iba, lah, mau, irur), eé (o-z), ee (l-z, l-z, l-z, l-z, l-z, l-z, leko, leko, mend), eez (ida), eré (a-zo, a-zo, a-zo, irur, z-p), ere (ahe, ahe, ainh, ainh, aiz, a-m, aha, arn, beho, eih, ame, ame, ame, anh, anh, anh, a-zo, arh, arh, arh, arm, arm, arm, arü, arü, arü, arü, ürr, ürr, ürr, ürr, ürr, a-s, a-s, a-s, a-s, azk, azk, azk, azk, azka, azka, azka, aih, aih, aih, beh, b-l, b-l, b-l, b-l, b-l, ban, bar, bar, bard, bard, be-l, be-l, be-l, be-l, be-l, bit, bid, bida, bida, bida, lek, lek, lek, lek, d-s, d-s, d-s, d-s, buz, g-zo, g-zo, g-zo, soh, soh, soh, soh, soh, sarr, sarr, sarr, zib, zib, zib, zib, zib, d-b, ünd, ünd, ünd, esp, esp, esp, esk, esk, esk, ezt, ezt, etxa, etxa, gab, gab, gab, gab, gab, gar, gar, gar, gar, gar, gar, garr, g-i, g-i, g-i, g-i, get, hel, hel, hel, hal, hal, hal, hal, hal, hal, hal, haz, haz, haz, haz, haz, haz, hoz, hoz, hoz, hoz, hoz, hoz, ida, ida, ida, ida, ida, ida, iho, iru, iru, izp, izp, izt, its, its, jat, jat, jat, jat, jat, jat, jat, jats, jats, jats, jut, jut, jut, jut, jut, laka, laka, laka, lekb, lekb, lekb, lekb, lan, lan, lan, lan, larz, larz, larz, larr, larr, larr, larr, lar, lar, lar, lar, lar, las, las, ald, ald, ald, ald, osp, osp, l-a, l-a, l-a, l-a, l-a, l-a, l-a, l-a, l-o, luh, luk, luk, mak, mak, meh, meh, meh, meh, meh, mend, mit, mit, mit, mit, mit, mit, mug, mug, müs, müs, ora, urd, urd, ost, ort, ort, izu, izu, izu, pag, pag, pag, arro, s-g, s-g, s-g, s-g, s-g, s-g, d-l, d-l, d-l, d-l, d-i, sen, sen, sen, sen, sen, sen, sar, sar, sar, sar, sar, zal, dono, dono, bai, bai, dona, dona, dona, dona, dom, dom, dom, dom, dom, dom, dom, suh, suh, u-g, u-h, u-h, u-h, u-h, u-h, u-h, ure, urr, urr, urr, mil, mil, bil, bil, bil), erek (bil), héré (hen, hen), here (its)

ereitze (1): ereïtciac (s-g)

eretze (1): eretcila (urd)

eriaro (1): Eriaro (mil)

erne (9): érné (ain), erné (hen), ernéric (a-z), erne (ahe, lar, hir, sar, urr), erneric (uzt)

erori (108): éoï (alt, etx), éoi (g-z, ath), éoy (hau), érori (a-z, ito, lah, l-s, mug, ur-p, z-p), éroriçienn (hen), érorissien (bun), érrori (ain, iba), eoï (alo, l-z), eori (arb, etxa), eroï (leko), eroi (urk), erori (ahe, ainh, a-m, ame, amo, anh, a-zo, arr, arm, eih, ürr, a-s, azk, beh, b-l, bar, bard, bas, be-l, bid, bir, lek, bes, buz, g-zo, sarr, zib, d-b, ünd, esk, gab, gar, hel, hal, haz, hoz, ida, ilh, iru, its, jat, jut, l-b, laka, larz, las, osp, l-a, luk, mak, mau, men, mend, mit, müs, arro, s-g, d-g, d-i, sen, sar, dono, bai, dona, suh, ure, mil, bil), eroric (u-g), eroriciren (ort), erorizien (zur), eroriziren (aiz, beho), erortcen (esp, get, lar, don), erortsen (luh), erortzen (azka, gam, iri, l-o, d-1), errori (arü), herrori (aha) errabiatu (8): arrabiatu (meh), errabiatiac (iho), errabiatiez (ilh, l-b), errabiatu (haz, zal), errabiatuya (mug), errabiatyuez (mak)

erran (376): éraiten (kan, kan, etx, lig, l-s, l-s, o-z, irur, irur), éraitencisun (etx), éraiten (o-z, z-p, z-p), eraiten (alo, alo, g-z, g-z, ünd, hau, ida, ida, lig, l-z, l-z, l-o, müs, müs, urd), eraiten (a-m, anh, arü, iri, lak, lak, l-a, l-a, l-o, l-o, luh, luh, luh, mar, mau, mau, d-l, 
d-g, dom, dom), éran (kan, etx, lig, o-z, irur, irur, z-p), erana (d-l), érantsen (l-s), eraten (azk, azk, zib, zib, d-l), ératen (ur-p), erayten (bil, bil), érraïten (ain, ain, bit, bit, don), érraiten (iba, don), érran (ain, arr, bun, iba, ito), érrana (ain), érrann (bit), eran (alo, anh, a-m, azk, g-z, lak, l-z, l-a, l-o, l-o, mar, müs, pag, d-g, zal, dom, urk, ur-p, bil), erréitén (ost), erraïteko (garr), erraïten (aha, aint, aiz, eih, zar, amo, anh, a-z, arb, arb, a-zo, arr, arr, arü, altz, beh, beh, be-l, be-l, bun, buz, buz, soh, sarr, sarr, ünd, gam, gam, gar, gar, gar, garr, garr, hau, ida, iru, ito, jats, jats, l-b, l-b, laka, lekb, lah, lah, lar, ora, pag, arro, s-g, s-g, sen, don, zal, bai, ath, ath, ure, ure), erréiten (ost), erraitentzuen (aiz), erraiteco (lar), erraiten (ainh, ainh, alt, alt, ame, amo, arn, a-z, a-zo, a-zo, beho, ürr, ürr, azka, altz, beh, b-l, b-l, ban, bar, bar, bas, bas, bid, bid, lek, lek, bun, d-s, g-zo, g-zo, g-zo, soh, d-b, d-b, esp, esp, esk, esk, ezt, ezt, gam, g-i, g-i, hal, hal, haz, hoz, hoz, iba, iho, iho, ilh, ilh, iri, iri, iru, izp, izp, izt, ito, its, its, jat, jat, jut, jut, laka, larz, larr, larr, lar, lar, las, las, ald, ald, ald, osp, mak, mak, mar, leko, men, men, mend, mit, mit, ora, ort, ort, arro, d-g, sen, hir, hir, hir, sar, zal, dona, dona, suh, suh, suh, u-g, u-g, mil, mil), erraitera (b-l), erraitia (osp), erran (aha, aint, arn, beho, eih, zar, ahe, ainh, alt, ame, amo, a-z, arb, a-zo, arh, arm, arü, ürr, a-s, altz, aih, b-l, ban, bar, bard, bas, be-l, bid, bida, bir, lek, bes, d-s, buz, soh, sarr, d-b, ünd, esp, esk, ezt, etxa, gab, garr, garr, g-i, get, get, hel, hal, haz, hau, iho, ilh, iri, iri, iru, iru, izt, its, jat, jats, jut, jut, jut, l-b, laka, lekb, lig, lah, lan, larr, lar, las, osp, luk, mak, mau, meh, leko, leko, leko, men, mend, mit, mug, ora, urd, urd, ort, izu, pag, pag, arro, s-g, d-l, $\mathrm{d}-\mathrm{i}$, sen, sar, dono, bai, dona, ath, irur, u-g, u-h, ure, urr, uzt, mil), Erran (a-z, a-z, a-z), errana (bida, zib, zib, zib, lar, s-g), erranari (sar), errandelaco (g-zo), errane (ost), erranera (hal, jat), errantendio (ur-p), errantzeen (larz), errantziezun (izp), erranzioten (zur), erratènn (hen), erraténn (hen, hen), errate (bida), erraten (aiz, ahe, ame, aih, aih, bida, bir, bir, zib, get, get, get, haz, meh, meh, leko, sar, dono, dono, ur-p, urr, urr, uzt), errayten (a-s, etxa, pag), erreiten (bard, bard, larz), erten (zar, arh, arh, arm, arm, azka, ban, bes, bes, d-s, gab, gab, hel, lan, lan, lan, luk, luk, mend, mug, izu, izu, d-i, d-i, zur, bai, u-h, u-h, urk, urk), ertendaco (mug), ertentzuin (zur), ertenzien (azka), irten (hel), érten (aint), irten (a-m, aha), ertenzeen (belo)

erranfren (2): erranfrena (ort, ort)

errankizun (4): errankiçuna (altz, altz), errankizuna (sar, sar)

erranpen (2): erranpena (dona, dona)

errantxu (4): érantchoua (etx, etx), erantchoua (l-z, l-z)

erreboltatu (1): erreboltatiez (gab)

erreka (4): erreka (azka, esp, gam, zur)

errekeitu (1): erekeitiac (urd)

errekolta (2): errecolta (bas), errekolten (garr)

errekuntratu (2): errecountratu (a-zo, g-zo)

errendatu (1): errendatcen (altz)

errepira (10): érépia (kan), erephea (dom), erpira (mug), errépéa (l-b), errepea (ilh), errephea (hel), errephera (ame), errepila (ur-p), errepira (bir, luk)

erresurtsa (1): eresoursa (1-a)

erretenitu (5): éréténitcen (hau), érétinitsën (g-z), eretenitzen (a-m, l-a), erretenitzentu (mit) erretiratu (9): érrétiatouic (iba), eretiatuic (hau), erretiatiak (gam), erretiatu (bard), erretiratiac (dona), erretiratou (jut), erretiratu (azka, mend), erretiratuac (dono) 
errezibitu (1): errezeïtu (garr)

erromes (11): érroméssac (ain), erroméçac (hen), erromech (sen), erromes (esp), erromesac (its), erromesagoak (esp), erromesak (bida, esp, esp), erromesen (bida), errumesago (arn)

ertankara (1): ertankara (zib)

eskaini (4): escaintsen (luh), eskaïni (arb, arr), eskaigni (bida)

eskapatu (47): echkapatzen (aha), éscapatsen (ito), éskapatzén (don), ejpacatcen (iba), escahatcen (arü), escapatcen (alt, amo, b-l, etx, hen, larr, müs, u-g, z-p), escapatsën (g-z), escapatzen (ezt, lekb, o-z), eskapatcen (altz, mar, d-g, ath), eskapatzen (ürr, mit), espacatcen (arm, izu, d-i), espacatzen (iri), espakatzen (d-b, mil), espekatzenzela (zur), ezcapatcen (sarr, ünd, etxa, g-i, ida, l-z), ezcapatzen (esk, gar), ezkapatzen (arh, garr, laka, u-h, bil), ezpakatzen (aiz, l-o)

eskas (2): eskasian (arb, arr)

eskernio (4): eskerniouago (gar), eskerniouan (gar, gar, gar)

eskolatu (1): ezkolatu (garr)

eskusatu (1): esquisatu (urr)

eslaio (1): eslayo (beho)

esne (147): échné (ito), éjné (ost), ésné (bun), ésnia (don), éssnia (bit), echnea (sen), ejsne (ban), esné (aint, zar, alt, kan, g-z, l-s, mau, o-z, z-p), esnéa (ain, hen), esne (ahe, ainh, amo, b-l, eih, zib, d-b, iho, ilh, l-b, lak, lekb, lan, larz, lar, l-a, mar, ora, izu, pag, d-l, hir, u-h), esnea (bida, iri, izt, its, luh, leko, mug, ort, sar, dona, uzt), esnetic (bir), esnia (a-m, anh, arn, a-z, arb, arr, arh, arm, azk, azka, aih, bas, beho, bid, lek, bes, buz, g-zo, esp, ezt, gab, gam, get, hel, hal, haz, hoz, iru, izp, jat, jats, jut, lah, las, ald, luk, mak, meh, mend, d-g, zur, dono, bai, dom, suh, u-g, urk, urr, mil), ezné (altz, hau, lig, larr), ezne (aiz, alo, arü, ürr, bar, bard, be-l, d-s, soh, sarr, ünd, esk, etx, etxa, gar, g-i, ida, laka, osp, l-z, l-o, men, mit, müs, urd, arro, s-g, d-i, zal, ath, irur, ure, bil), eznez (garr), eznia (ame, a-zo, a-s, beh, ur-p), ejnia (aha), esné (aint), esne (eih)

Espainia (3): Españako (iho), Espagnaco (lan), Espagnalaco (l-a)

estainu (3): estaïnu (arr), estainubat (arb), esteynu (mil)

estali (70): echtalia (sen), echtaliacen (aha), éstalia (ain, kan), ejstalia (ban), esjtali (iba), est'aliacéna (hen), estalia (aint, arn, eih, zar, ahe, ahe, ame, anh, arb, arr, azk, bas, bida, bir, lek, bes, d-s, zib, esp, ezt, get, hel, hal, haz, hoz, iri, iru, izp, izt, its, jat, jats, lak, lah, lan, lar, las, ald, luh, luk, mak, mar, meh, ort, d-l, d-i, hir, sar, zur, dono, bai, dona, ure, ur-p, urr, uzt, mil), estaliacen (leko, mug), estaliazen (aiz, a-s, azka), estalii (urk), estallia (bard), estealia (gam), eztaliric (irur)

estranier (82): ésstranyérrac (bit), éstranyérék (don), éstranyérrék (ost), ejtranyer (iba), estranier (d-g), estranierec (d-g), estrandeye (ur-p), estrandjerek (laka), estrangé (altz), estrangeec (arü), estrangerec (soh, mar, d-i), estrangerhec (aiz), estranjéec (g-z), estranjérec (z-p), estranjec (hau), estranjeek (sarr, ünd, men), estranjerec (beho, esk, ida, osp, müs), estranjerek (alo, bar, mil, bil), estranjerrak (arh), estranjerrec (be-l), estranjerrek (u-g), estranjerrrec (larr), estrantgerec (etxa), estranyérac (l-s), estranyèrrec (bun), estranyérrec (hen), estranyer (ame, haz, lah), Estranyer (haz), estranyerac (its), estranyerec (anh, bid, bir, eih, get, iru, lekb, ald, mak, mend, ora, dom), estranyerek (a-m, bard, zur, dono, urk), estranyerrac ( $u-h)$, estranyerrec (a-zo, azka, g-zo, gab, jut, lan, larz, izu, dona, suh), estranyerrek (a-s, buz, gam, iba, ilh, jats, l-b, las), estrayer 
(u-g), Eztranyer (b-l), eztranyerrek (beh)

estremitate (1): estremitatean (gam)

eta (3158): cêneta (1-b), cereneta (urd), noizeta (arm, b-l), Noizeta (hoz), éta (ain, ain, ain, ain,

ain, ain, ain, ain, ain, ain, ain, ain, ain, ain, ain, ain, ain, ain, ain, ain, aint, zar, zar, zar, zar, zar, zar, zar, zar, zar, zar, zar, zar, zar, zar, zar, zar, zar, zar, alt, alt, alt, alt, alt, alt, alt, alt, alt, alt, alt, alt, alt, a-z, a-z, a-z, a-z, a-z, a-z, a-z, a-z, a-z, a-z, a-z, a-z, a-z, a-z, a-z, a-z, a-z, a-z, a-z, a-z, a-zo, beh, bit, bit, bit, bit, bit, bit, bit, bit, bit, bit, bit, bit, bit, bit, bit, bit, bit, bit, bit, bit, bit, bit, bun, bun, bun, bun, bun, bun, bun, bun, bun, bun, bun, bun, bun, bun, bun, bun, bun, bun, bun, bun, bun, kan, kan, kan, kan, kan, kan, kan, kan, kan, kan, kan, kan, kan, kan, kan, kan, kan, kan, kan, kan, ünd, etx, etx, etx, etx, etx, etx, etx, etx, etx, etx, etx, etx, etx, etx, etx, etx, etx, hau, hau, hau, hau, hen, hen, hen, hen, hen, hen, hen, hen, hen, hen, hen, hen, hen, hen, hen, hen, hen, hen, hen, hen, hen, iba, iba, iba, iba, iba, iba, iba, iba, iba, iba, iba, iba, iba, iba, iba, iba, iba, iba, iba, iba, ilh, ito, ito, ito, ito, ito, ito, ito, ito, ito, ito, ito, ito, ito, ito, ito, ito, ito, ito, lah, lah, lah, lah, lah, lah, lah, lah, lah, lah, lah, lah, lah, lah, l-s, l-s, l-s, l-s, l-s, l-s, l-s, l-s, l-s, l-s, l-s, l-s, l-s, l-s, l-s, l-s, l-s, l-s, urd, ost, ost, ost, ost, ost, ost, ost, ost, ost, ost, ost, ost, ost, ost, o-z, o-z, o-z, o-z, don, don, don, don, don, don, don, don, don, don, don, don, don, don, don, don, don, don, don, ath, ath, ath, ath, ath, ath, ath, ath, ath, ath, ath, ath, ath, ath, ath, ath, ath, ath, ath, ath, ath, ath, irur, irur, irur, irur, irur, irur, irur, irur, irur, irur, z-p, z-p, z-p, z-p, z-p, z-p, z-p, z-p, z-p, z-p), été (bun), étaaré (gam), étaré (zar, zar), et (ainh, aint, alo, bit, bir, bir, ünd, garr, its, lig, l-o, leko, leko, mug, arro, sen, sen, sen, sar, ur-p), eta (aiz, aiz, aiz, aiz, aiz, aiz, aiz, aiz, aiz, aiz, aiz, aiz, aiz, aiz, aiz, aiz, aiz, aiz, ain, ain, a-m, aha, aint, arn, beho, eih, zar, ahe, ahe, ahe, ahe, ahe, ahe, ahe, ahe, ahe, ahe, ahe, ahe, ahe, ahe, ahe, ahe, ahe, ahe, ahe, ainh, ainh, ainh, ainh, ainh, ainh, ainh, ainh, ainh, ainh, ainh, ainh, ainh, ainh, ainh, ainh, ainh, ainh, ainh, ainh, alt, alt, alt, alt, alo, alo, alo, alo, alo, alo, alo, alo, alo, alo, alo, alo, alo, alo, alo, alo, alo, alo, ame, ame, ame, ame, ame, ame, ame, ame, ame, ame, ame, ame, ame, ame, ame, ame, ame, ame, ame, ame, ame, ame, ame, ame, , , , , , amo, amo, amo, amo, amo, amo, amo, amo, amo, amo, amo, amo, amo, amo, amo, amo, amo, amo, amo, amo, amo, amo, anh, anh, anh, anh, anh, anh, anh, anh, anh, anh, anh, anh, anh, anh, anh, anh, anh, anh, anh, anh, anh, arb, arb, arb, arb, arb, arb, arb, arb, arb, arb, arb, arb, arb, arb, arb, arb, arb, arb, arb, arb, a-zo, a-zo, a-zo, a-zo, a-zo, a-zo, a-zo, a-zo, a-zo, a-zo, a-zo, a-zo, a-zo, a-zo, a-zo, a-zo, a-zo, a-zo, a-zo, a-zo, a-zo, arr, arr, arr, arr, arr, arr, arr, arr, arr, arr, arr, arr, arr, arr, arr, arr, arr, arr, arr, arr, arh, arh, arh, arh, arh, arh, arh, arh, arh, arh, arh, arh, arh, arh, arh, arh, arh, arh, arh, arh, arh, arh, arh, arh, arh, arm, arm, arm, arm, arm, arm, arm, arm, arm, arm, arm, arm, arm, arm, arm, arm, arm, arm, arm, arm, arm, arü, arü, arü, arü, arü, arü, arü, arü, arü, arü, arü, arü, arü, arü, arü, arü, arü, arü, arü, arü, ürr, ürr, ürr, ürr, ürr, ürr, ürr, ürr, ürr, ürr, ürr, ürr, ürr, ürr, ürr, ürr, ürr, ürr, ürr, ürr, ürr, ürr, a-s, a-s, a-s, a-s, a-s, a-s, a-s, a-s, a-s, a-s, a-s, a-s, a-s, a-s, a-s, a-s, a-s, a-s, a-s, a-s, a-s, azk, azk, azk, azk, azk, azk, azk, azk, azk, azk, azk, azk, azk, azk, azk, azk, azk, azk, azk, azk, azk, azk, azka, azka, azka, azka, azka, azka, azka, azka, azka, azka, azka, azka, azka, azka, azka, azka, azka, azka, azka, azka, azka, altz, altz, altz, altz, altz, altz, altz, altz, altz, altz, altz, altz, altz, altz, altz, altz, altz, altz, altz, altz, altz, altz, altz, altz, altz, altz, altz, altz, altz, aih, aih, aih, aih, aih, aih, aih, aih, aih, aih, aih, aih, aih, aih, aih, aih, aih, aih, aih, beh, beh, beh, beh, beh, 
beh, beh, beh, beh, beh, beh, beh, beh, beh, beh, beh, beh, beh, beh, beh, b-l, b-l, b-l,

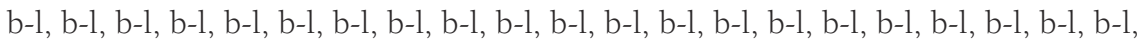
ban, ban, ban, ban, ban, ban, ban, ban, ban, ban, ban, ban, ban, ban, ban, ban, ban, ban, ban, ban, ban, bar, bar, bar, bar, bar, bar, bar, bar, bar, bar, bar, bar, bar, bar, bar, bar, bar, bar, bar, bar, bard, bard, bard, bard, bard, bard, bard, bard, bard, bard, bard, bard, bard, bard, bard, bard, bard, bard, bard, bard, bard, bard, bard, bas, bas, bas, bas, bas, bas, bas, bas, bas, bas, bas, bas, bas, bas, bas, bas, bas, bas, bas, be-l, be-l, be-l, be-l, be-l, be-l, be-l, be-l, be-l, be-l, be-1, be-l, be-l, be-l, be-l, be-l, be-l, be-l, be-l, be-l, be-l, be-l, be-l, bit, bid, bid, bid, bid, bid, bid, bid, bid, bid, bid, bid, bid, bid, bid, bid, bid, bid, bid, bid, bid, bid, bida, bida, bida, bida, bida, bida, bida, bida, bida, bida, bida, bida, bida, bida, bida, bida, bida, bida, bida, bida, bir, bir, bir, bir, bir, bir, bir, bir, bir, bir, bir, bir, bir, bir, bir, bir, bir, lek, lek, lek, lek, lek, lek, lek, lek, lek, lek, lek, lek, lek, lek, lek, lek, lek, lek, lek, lek, bes, bes, bes, bes, bes, bes, bes, bes, bes, bes, bes, bes, bes, bes, bes, bes, bes, bes, bun, bun, d-s, d-s, d-s, d-s, d-s, d-s, d-s, d-s, d-s, d-s, d-s, d-s, d-s, d-s, d-s, d-s, d-s, d-s, d-s, d-s, d-s, d-s, buz, buz, buz, buz, buz, buz, buz, buz, buz, buz, buz, buz, buz, buz, buz, buz, buz, buz, buz, buz, kan, kan, g-z, g-z, g-z, g-z, g-z, g-z, g-z, g-z, g-z, g-z, g-z, g-z, g-z, g-z, g-z, g-z, g-z, g-z, g-zo, g-zo, g-zo, g-zo, g-zo, g-zo, g-zo, g-zo, g-zo, g-zo, g-zo, g-zo, g-zo, g-zo, g-zo, g-zo, g-zo, g-zo, g-zo, g-zo, g-zo, g-zo, soh, soh, soh, soh, soh, soh, soh, soh, soh, soh, soh, soh, soh, soh, soh, soh, soh, soh, soh, soh, soh, soh, soh, sarr, sarr, sarr, sarr, sarr, sarr, sarr, sarr, sarr, sarr, sarr, sarr, sarr, sarr, sarr, sarr, sarr, sarr, sarr, zib, zib, zib, zib, zib, zib, zib, zib, zib, zib, zib, zib, zib, zib, zib, zib, zib, zib, zib, zib, zib, zib, d-b, d-b, d-b, d-b, d-b, d-b, d-b, d-b, d-b, d-b, d-b, d-b, d-b, d-b, d-b, d-b, d-b, d-b, d-b, d-b, ünd, ünd, ünd, ünd, ünd, ünd, ünd, ünd, ünd, ünd, ünd, ünd, ünd, ünd, ünd, ünd, ünd, ünd, esp, esp, esp, esp, esp, esp, esp, esp, esp, esp, esp, esp, esp, esp, esp, esp, esp, esp, esp, esp, esp, esp, esp, esp, esk, esk, esk, esk, esk, esk, esk, esk, esk, esk, esk, esk, esk, esk, esk, esk, esk, esk, esk, esk, ezt, ezt, ezt, ezt, ezt, ezt, ezt, ezt, ezt, ezt, ezt, ezt, ezt, ezt, ezt, ezt, ezt, ezt, ezt, ezt, ezt, etx, etx, etxa, etxa, etxa, etxa, etxa, etxa, etxa, etxa, etxa, etxa, etxa, etxa, etxa, etxa, etxa, etxa, etxa, etxa, etxa, etxa, gab, gab, gab, gab, gab, gab, gab, gab, gab, gab, gab, gab, gab, gab, gab, gab, gab, gab, gab, gab, gab, gab, gab, gab, gam, gam, gam, gam, gam, gam, gam, gam, gam, gam, gam, gam, gam, gam, gam, gam, gam, gam, gam, gam, gar, gar, gar, gar, gar, gar, gar, gar, gar, gar, gar, gar, gar, gar, gar, gar, gar, gar, gar, gar, garr, garr, garr, garr, garr, garr, garr, garr, garr, garr, garr, garr, garr, garr, garr, garr, garr, garr, garr, garr, garr, garr, garr, garr, garr, garr, g-i, g-i, g-i, g-i, g-i, g-i, g-i, g-i, g-i, g-i, g-i, g-i, g-i, g-i, g-i, g-i, g-i, g-i, g-i, g-i, get, get, get, get, get, get, get, get, get, get, get, get, get, get, get, get, get, get, get, hel, hel, hel, hel, hel, hel, hel, hel, hel, hel, hel, hel, hel, hel, hel, hel, hel, hel, hel, hel, hel, hal, hal, hal, hal, hal, hal, hal, hal, hal, hal, hal, hal, hal, hal, hal, hal, hal, hal, hal, hal, hal, haz, haz, haz, haz, haz, haz, haz, haz, haz, haz, haz, haz, haz, haz, haz, haz, haz, haz, haz, haz, haz, haz, haz, hau, hau, hau, hau, hau, hau, hau, hau, hau, hau, hau, hau, hau, hau, hau, hau, hau, hoz, hoz, hoz, hoz, hoz, hoz, hoz, hoz, hoz, hoz, hoz, hoz, hoz, hoz, hoz, hoz, hoz, hoz, hoz, hoz, hoz, hoz, hoz, hoz, hoz, iba, ida, ida, ida, ida, ida, ida, ida, ida, ida, ida, ida, ida, ida, ida, ida, ida, ida, ida, ida, ida, ida, ida, ida, ida, iho, iho, iho, iho, iho, iho, iho, iho, iho, iho, iho, iho, iho, iho, iho, iho, iho, iho, iho, iho, ilh, ilh, ilh, ilh, ilh, ilh, ilh, ilh, ilh, ilh, ilh, ilh, ilh, ilh, ilh, ilh, ilh, iri, iri, iri, iri, iri, iri, iri, iri, iri, iri, iri, iri, iri, iri, iri, 
iri, iri, iri, iri, iri, iru, iru, iru, iru, iru, iru, iru, iru, iru, iru, iru, iru, iru, iru, iru, iru, iru, iru, iru, iru, iru, iru, izp, izp, izp, izp, izp, izp, izp, izp, izp, izp, izp, izp, izp, izp, izp, izp, izp, izp, izp, izp, izp, izp, izt, izt, izt, izt, izt, izt, izt, izt, izt, izt, izt, izt, izt, izt, izt, izt, izt, izt, izt, izt, izt, izt, its, its, its, its, its, its, its, its, its, its, its, its, its, its, its, its, its, its, its, its, its, jat, jat, jat, jat, jat, jat, jat, jat, jat, jat, jat, jat, jat, jat, jat, jat, jat, jat, jat, jat, jat, jats, jats, jats, jats, jats, jats, jats, jats, jats, jats, jats, jats, jats, jats, jats, jats, jats, jats, jats, jats, jats, jut, jut, jut, jut, jut, jut, jut, jut, jut, jut, jut, jut, jut, jut, jut, jut, jut, jut, jut, jut, jut, jut, jut, jut, l-b, l-b, l-b, l-b, l-b, l-b, l-b, l-b, l-b, l-b, l-b, l-b, l-b, l-b, l-b, l-b, l-b, l-b, l-b, lak, lak, lak, lak, lak, lak, lak, lak, lak, lak, lak, lak, lak, lak, lak, lak, lak, lak, lak, lak, laka, laka, laka, laka, laka, laka, laka, laka, laka, laka, laka, laka, laka, laka, laka, laka, laka, laka, laka, laka, laka, lekb, lekb, lekb, lekb, lekb, lekb, lekb, lekb, lekb, lekb, lekb, lekb, lekb, lekb, lekb, lekb, lekb, lekb, lekb, lekb, lig, lig, lig, lig, lig, lig, lig, lig, lig, lig, lig, lig, lig, lig, lig, lig, lig, lig, lig, lig, lig, lig, lah, lah, lah, lah, lah, lah, lah, lah, lan, lan, lan, lan, lan, lan, lan, lan, lan, lan, lan, lan, lan, lan, lan, lan, lan, lan, lan, lan, lan, larz, larz, larz, larz, larz, larz, larz, larz, larz, larz, larz, larz, larz, larz, larz, larz, larz, larz, larz, larz, larz, larz, larz, larr, larr, larr, larr, larr, larr, larr, larr, larr, larr, larr, larr, larr, larr, larr, larr, larr, larr, larr, larr, lar, lar, lar, lar, lar, lar, lar, lar, lar, lar, lar, lar, lar, lar, lar, lar, lar, l-s, l-s, las, las, las, las, las, las, las, las, las, las, las, las, las, las, las, las, las, las, las, las, las, ald, ald, ald, ald, ald, ald, ald, ald, ald, ald, ald, ald, ald, ald, ald, ald, ald, osp, osp, osp, osp, osp, osp, osp, osp, osp, osp, osp, osp, osp, osp, osp, osp, osp, osp, osp, l-z, l-z, l-z, l-z, l-z, l-z, l-z, l-z, l-z, l-z, l-z, l-z, l-z, l-z, l-z, l-z, l-z, l-z, l-a, l-a, l-a, l-a, l-a, l-a, l-a, l-a, l-a, l-a, l-a, l-a, l-a, l-a, l-a, l-a, l-a, l-a, l-o, l-o, l-o, l-o, l-o, l-o, l-o, l-o, l-o, l-o, l-o, l-o, l-o, l-o, l-o, l-o, l-o, l-o, luh, luh, luh, luh, luh, luh, luh, luh, luh, luh, luh, luh, luh, luh, luh, luh, luh, luh, luh, luh, luk, luk, luk, luk, luk, luk, luk, luk, luk, luk, luk, luk, luk, luk, luk, luk, luk, mak, mak, mak, mak, mak, mak, mak, mak, mak, mak, mak, mak, mak, mak, mak, mak, mak, mak, mak, mar, mar, mar, mar, mar, mar, mar, mar, mar, mar, mar, mar, mar, mar, mar, mar, mar, mar, mar, mar, mar, mau, mau, mau, mau, mau, mau, mau, mau, mau, mau, mau, mau, mau, mau, mau, mau, mau, mau, mau, mau, meh, meh, meh, meh, meh, meh, meh, meh, meh, meh, meh, meh, meh, meh, meh, meh, meh, meh, meh, meh, meh, meh, meh, leko, leko, leko, leko, leko, leko, leko, leko, leko, leko, leko, leko, leko, leko, leko, leko, leko, leko, leko, men, men, men, men, men, men, men, men, men, men, men, men, men, men, men, men, men, men, men, mend, mend, mend, mend, mend, mend, mend, mend, mend, mend, mend, mend, mend, mend, mend, mend, mend, mend, mend, mend, mit, mit, mit, mit, mit, mit, mit, mit, mit, mit, mit, mit, mit, mit, mit, mit, mit, mit, mit, mit, mit, mug, mug, mug, mug, mug, mug, mug, mug, mug, mug, mug, mug, mug, mug, mug, mug, mug, mug, mug, müs, müs, müs, müs, müs, müs, müs, müs, müs, müs, müs, müs, müs, müs, müs, müs, müs, müs, müs, müs, müs, müs, müs, ora, ora, ora, ora, ora, ora, ora, ora, ora, ora, ora, ora, ora, ora, ora, ora, ora, ora, ora, ora, urd, urd, urd, urd, urd, urd, urd, urd, urd, urd, urd, urd, urd, urd, urd, urd, urd, urd, urd, ost, ost, ost, ost, ost, ost, o-z, o-z, o-z, o-z, o-z, o-z, o-z, o-z, o-z, o-z, o-z, o-z, o-z, o-z, o-z, o-z, o-z, o-z, o-z, ort, ort, ort, ort, ort, ort, ort, ort, ort, ort, ort, ort, ort, ort, ort, ort, ort, ort, ort, ort, ort, izu, izu, izu, izu, izu, izu,

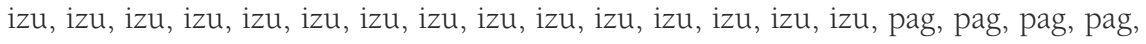
pag, pag, pag, pag, pag, pag, pag, pag, pag, pag, pag, pag, pag, pag, pag, arro, arro, 
arro, arro, arro, arro, arro, arro, arro, arro, arro, arro, arro, arro, arro, arro, arro, arro, arro, arro, arro, arro, s-g, s-g, s-g, s-g, s-g, s-g, s-g, s-g, s-g, s-g, s-g, s-g, s-g, s-g, s-g, s-g, s-g, s-g, s-g, s-g, s-g, d-l, d-l, d-l, d-l, d-l, d-l, d-l, d-l, d-l, d-l, d-l, d-l, d-l, d-l, d-l, d-l, d-1, d-l, d-l, d-l, d-g, d-g, d-g, d-g, d-g, d-g, d-g, d-g, d-g, d-g, d-g, d-g, d-g, d-g, d-g, d-g, d-g, d-g, d-g, d-g, d-g, d-g, d-i, d-i, d-i, d-i, d-i, d-i, d-i, d-i, d-i, d-i, d-i, d-i, d-i, d-i, d-i, d-i, d-i, d-i, d-i, d-i, sen, sen, sen, sen, sen, sen, sen, sen, sen, sen, sen, sen, sen, sen, sen, sen, sen, sen, sen, sen, sen, sen, sen, sen, don, don, don, don, hir, hir, hir, hir, hir, hir, hir, hir, hir, hir, hir, hir, hir, hir, hir, hir, hir, hir, hir, hir, hir, sar, sar, sar, sar, sar, sar, sar, sar, sar, sar, sar, sar, sar, sar, sar, sar, sar, sar, sar, sar, sar, zal, zal, zal, zal, zal, zal, zal, zal, zal, zal, zal, zal, zal, zal, zal, zal, zal, zal, zal, zal, zal, zal, zur, zur, zur, zur, zur, zur, zur, zur, zur, zur, zur, zur, zur, zur, zur, zur, zur, zur, zur, zur, zur, dono, dono, dono, dono, dono, dono, dono, dono, dono, dono, dono, dono, dono, dono, dono, dono, dono, dono, dono, bai, bai, bai, bai, bai, bai, bai, bai, bai, bai, bai, bai, bai, bai, bai, bai, bai, bai, bai, bai, dona, dona, dona, dona, dona, dona, dona, dona, dona, dona, dona, dona, dona, dona, dona, dona, dona, dona, dona, dona, dona, dona, dona, dom, dom, dom, dom, dom, dom, dom, dom, dom, dom, dom, dom, dom, dom, dom, dom, dom, dom, suh, suh, suh, suh, suh, suh, suh, suh, suh, suh, suh, suh, suh, suh, suh, suh, suh, suh, suh, suh, suh, ath, ath, irur, irur, irur, irur, irur, irur, irur, irur, irur, irur, irur, irur, irur, u-g, u-g, u-g, u-g, u-g, u-g, u-g, u-g, u-g, u-g, u-g, u-g, u-g, u-g, u-g, u-g, u-g, u-g, u-g, u-g, u-g, u-h, u-h, u-h, u-h, u-h, u-h, u-h, u-h, u-h, u-h, u-h, u-h, u-h, u-h, u-h, u-h, u-h, u-h, u-h, u-h, u-h, u-h, u-h, urk, urk, urk, urk, urk, urk, urk, urk, urk, urk, urk, urk, urk, urk, urk, urk, urk, urk, urk, urk, ure, ure, ure, ure, ure, ure, ure, ure, ure, ure, ure, ure, ure, ure, ure, ure, ure, ure, ure, ure, ure, ur-p, ur-p, ur-p, ur-p, ur-p, ur-p, ur-p, ur-p, ur-p, ur-p, ur-p, ur-p, ur-p, ur-p, ur-p, ur-p, ur-p, ur-p, ur-p, ur-p, ur-p, urr, urr, urr, urr, urr, urr, urr, urr, urr, urr, urr, urr, urr, urr, urr, urr, urr, urr, urr, urr, urr, urr, urr, urr, uzt, uzt, uzt, uzt, uzt, uzt, uzt, uzt, uzt, uzt, uzt, uzt, uzt, uzt, uzt, uzt, uzt, uzt, uzt, uzt, mil, mil, mil, mil, mil, mil, mil, mil, mil, mil, mil, mil, mil, mil, mil, mil, mil, mil, mil, mil, mil, mil, bil, bil, bil, bil, bil, bil, bil, bil, bil, bil, bil, bil, bil, bil, bil, bil, bil, bil, bil, bil, z-p, z-p, z-p, z-p, $z-p, z-p, z-p, z-p, z-p)$, Eta (garr), etaé (lig), etaare (gam, gam), etae (men), etaere (gam), etare (arü, arü, garr, ure, ure, ure), etea (alt, soh), ita (dono, dono), ta (a-zo, be-l, soh, ilh, larr, u-h, urk), zeta (aha, urd)

eta ere (3): étaére (ain), etare (eih, eih)

etorri (15): éthorrico (ain), étorrikoc (ur-p), ethorico (azk), ethorrico (get, urr), ethorriko (ahe, ame, bida, zib, d-l, sar), ethortcen (ort), ethortcerat (sen), etorrico (hen), etorricouc (bir)

etxe (445): éché (ur-p, ur-p, ur-p), étché (ain, ain, ain, bit, bun, bun, bun, kan, hau, hau, iba, ito, lah, ost, don), étchéic (iba), étchènn (bit, bit), étchérïc (ost), étche (garr), étcheïc (ito), étcheric (z-p), étchiaïn (bit, iba, ost), étchiaën (hau), echeik (1-b), etché (aint, zar, zar, zar, ainh, a-z, altz, altz, beh, kan, g-z, etx, hen, izt, l-b, lah, l-s, l-s, mau, mit, mug, don, dona, ath, z-p, z-p), etchéic (a-z, l-s), etchian (gab), etchéaren (lah), etchéarenn (hen), etchaïtcinian (larr), etchéchipignoat (a-zo), etchéic (g-z), etchéik (ath), etchéric (ainh, bit, hen), etche (aha, ahe, ainh, aiz, alt, alo, alo, a-m, ame, ame, amo, anh, arb, arb, arn, arr, arr, arh, arh, arh, arm, arü, ürr, a-s, azk, azka, aih, beho, b-l, b-l, ban, bar, bar, bard, bas, bas, be-l, be-l, bid, bida, bida, bida, bir, bir, bes, bes, d-s, d-s, buz, buz, 
eih, g-z, g-zo, soh, soh, sarr, d-b, ünd, esp, esp, esk, esk, ezt, etx, etx, etxa, etxa, gab, gam, gam, gar, gar, garr, g-i, get, get, hel, hal, hoz, hoz, ida, iho, ilh, iri, iri, iri, iru, iru, izp, izp, izt, its, its, jat, jats, jut, jut, lak, laka, lekb, lekb, lig, lig, lan, larz, larz, larr, las, las, ald, ald, l-z, l-z, l-z, l-a, l-o, l-o, luh, luh, luk, mak, mar, mar, mau, meh, meh, leko, men, mend, müs, müs, ora, urd, o-z, o-z, ort, izu, izu, pag, arro, arro, s-g, d-l, d-l, d-g, d-g, d-i, d-i, sen, don, hir, sar, zal, zur, zur, dono, dono, bai, dona, dom, dom, suh, irur, irur, u-g, u-h, u-h, u-h, urk, urk, ure, ure, urr, uzt, mil, mil, bil, bil), Etche (aih, ban, lek, zib, haz), etcheïc (a-zo, g-zo, gab, hel, pag), etche'ic (ban), etcheïk (beh, gar), etchean (haz, sar, urk), etchearen (ahe, ame, arn, lek, get, hal, haz, hoz, iru, izt, its, jat, lak, lan, luh, luk, leko, mug, ort, hir, sar, dono), etcheat (garr, mend), etcheate (izu), etchebat (larr), etcheco (bes, mend), etcheen (d-l, sar), etcheic (bid, osp, bai), etcheik (altz, a-m, bard, garr, ilh, leko, men), etchen (b-l, haz, ora, pag), etchera (zal), etcherat (arm, bar, zib), etcheric (amo, anh, arm, arü, beho, eih, ürr, azk, azka, b-l, be-l, lek, soh, esk, ezt, etxa, g-i, hal, haz, ida, iho, jat, lak, laka, lekb, lan, lar, las, l-a, luk, mak, mar, mau, mit, mug, müs, ora, urd, o-z, ort, arro, s-g, d-i, sen, hir, zal, suh, u-g, ure, urr), etcherik (aiz, ahe, alo, arn, a-s, bar, d-s, buz, sarr, zib, d-b, jats, l-o, luh, sar, bil), etcherric (uzt), etcherrik (ünd), etchetan (alt, altz), etchetara (ame), etchez (bida), etchiaan (hel), etchia-an (aih), etchiain (gam, jats), etchiaen (a-zo, arü, beh, g-zo, sarr, d-b, ünd, lig, men), etchiain (azka, bid), etchian (bard, bir, d-s, zib, esp, ilh, l-b), etchiaren (a-m, amo, anh, arb, arr, arm, eih, ürr, a-s, azk, zib, esp, g-i, ida, iho, izp, jut, laka, larz, ald, mak, meh, mit, ora, s-g, bai, dona, dom, irur, urr, mil), etchien (bas, luh), etciaren (kan), hetcheat (aha)

etxeki (1): etchekitcentu (pag)

etxeño (1): etchégno (a-z)

etxeratu (1): etcheratu (mug)

etxetto (2): etchetto (l-a, urd)

etxola (3): etcholabat (lar), etcholaen (osp), etcholat (osp)

etzan (141): étçana (ain, beh), étçanic (bit, hau), étçanik (don), étsana (kan), étsanic (l-s), etçana (a-m, anh, a-z, azk, b-l, bard, bida, eih, esp, ilh, izt, its, jut, l-b, lan, mak, leko, mug, ora, ort, d-i, sen, hir, suh), etçanic (aha, aint, zar, ainh, alt, amo, a-zo, arm, arü, altz, be-l, g-zo, soh, etx, etxa, gab, g-i, iba, ida, larr, lar, las, osp, l-z, l-a, mar, mau, müs, urd, izu, pag, arro, s-g, zal, ure, z-p), etçanik (laka, men, d-g, bil), etcinndouä (hen), etsana (ban, ald, luh, mend, ost, dono), etsanic (bun, g-z, lig, o-z, irur), etxana (arr), etzana (ahe, arb, azka, aih, bas, bid, bir, lek, bes, d-s, d-b, gam, get, haz, iho, iri, iru, izp, jats, lak, lah, larz, luk, meh, d-l, sar, zur, bai, dona, dom, ur-p, urr, uzt, mil), etzanac (ame), etzanic (ame, arh, esk, ezt, hoz, lekb, mit, u-g, u-h), etzanik (aiz, alo, ürr, a-s, bar, buz, sarr, ünd, gar, garr, l-o), etziña (zib)

ez (1189): eez (ida), écititcien (alt), és (bit, kan, kan, ost), ésdéçatènn (bit), ésdiénian (l-s), ésdou (kan), èsdouteniann (bit), éspitsutén (ito), étçakién (hau), étçakicièn (bit), étçakitén (ain), étçoutén (ain), étçoutènn (bit), étçu (beh), étçutien (hau), étci (iba, ost), étciakicién (ost), étcicien (iba, z-p), étciticién (ost), étcitouén (ain), étsakiten (kan, ito), étsissien (bun), étsitouen (kan), étsouten (bun, kan), étsusten (ito), étsutén (l-s, l-s), éz (ain, ain, ain, ain, bun, iba, don, don, don, don, don), ézçauténn (hen), ézdou (don), ézituzten (ur-p), es (alt, ame, ürr, esp, esp, get, ito, l-s, mau, mit, ur-p, ur-p), esbeitouté (kan), esdésaten (l-s), esdazaten (l-o), esdia (l-s), esdu (l-s), eshakicien 
(bard), espadou (g-zo), espadu (a-zo), espeïtsakiten (bun), espeïtu (alt, ora), espeittuke (altz), espeitié (o-z), espeitu (amo, arü, soh, hau, lig, l-a, l-o, mar, mau, z-p), espeytu (pag, bil), essassie (o-z), essassu (lig, irur), esta (arü, eih, ünd, lig, pag, irur), estéçatén (ito), estécën (g-z), estécén (hau), estacen (larr), estécen (alt, z-p), estadin (lar), esteçan (ora), esteçaten (a-zo, mar), estecen (ainh, arü, sarr, ünd, mau, o-z, pag, ath, bil), estenian (ainh), estesen (ürr), estesssen (lig), estezaten (lekb), estezen (l-a), estianian (ath), estiénian (bun, ost), estinïn (g-z), estirenian (larr), estou (lekb), estoutino (lekb), estu (amo, ito, ora, z-p), estuçu (ainh, g-z, etx, l-a, mau, ath), estuenia (ur-p), estuténian (ito), estutienian (irur), estzi (d-s), etçakicien (ainh, altz, ida, larr, l-z, mar, men, arro, bil), etçakien (soh, g-i, urd, pag, s-g, zal), etçakiten (a-m, zar, a-z, ilh, l-b, lan, las, müs, izu, ure), etçakizien (etx, laka, ath), etçaquicien (alt, mau), etçaquien (be-l), etçititçun (laka), Etçoutén (ain), etçoutènn (bit), etçouten (aha, aint, zar, ora), Etçouten (ora, sen, u-g), Etçutènn (bit), etçuten (a-m, a-z, bida, ilh, l-b, lar, las, mar, leko, müs, izu, d-g, ure), Etçuten (aint, eih, zar, a-z, ilh, l-b, las, müs, izu, ure), etçutian (g-i, müs, s-g), etçutien (soh, zal), etçutin (arü, pag), etçuzten (zar, a-z, ilh, izu), etcakiten (izt), etcen (uzt), etceren ( $g-i)$, etchekitcen (arü, osp, s-g), etchekiten (bar, ida), etchekitzen (sarr, ünd, gar), etci (a-zo, bard, g-zo, hoz, mend, d-g), etciakicien (hoz), Etcién (hau), etcicién (ost), Etcicién (iba), etcicien (ainh, a-m, a-zo, altz, altz, bard, bard, g-zo, hoz, ida, laka, larr, mar, mau, men, mend, arro, bil), Etcicien (ainh, alt, a-zo, beh, g-zo, etx, hoz, ida, laka, larr, l-z, mar, mau, men, mend, arro, d-g, ath, bil, z-p), etcicin (a-zo), etcien (arü, be-l, sarr, g-i, hau, urd, pag, s-g, zal, ath), Etcien (arü, be-l, soh, sarr, g-i, pag, s-g, zal), etciren (soh), Etcisiën (g-z), Etcissién (ost), etcitïcun (etx), etcitcien (beh), Etcitcien (etxa), etcitcin (g-zo), etcitian (ure), etcitiçun (mau), etciticien (ainh, altz, etx, l-z, men, arro, ath), etcitin (arr, las), etcititçun (ida, l-z), etcititcien (larr, bil), etcituen (izt), etcitusten (arb), etcitzien (hoz), etciuzten (ort, u-g), etcuzten (l-b), etsakien (lig, irur), etsén (ito), etsaquiciën ( $g-z)$, etsaquien (o-z), etsein (urd), etsicien (g-z), Etsicien (bard), etsien (lig, o-z, irur), Etsien (lig, urd, o-z, irur), etsitiçün (g-z), etsou (bun), Etsoutén (bun), Etsutén (ito), Etsuten (kan, l-s), etsutian (lig, irur, z-p), etsutien (o-z), etuten (dono), etxaquiten (arr), etz (ünd), Etz (urk), etzüzten (d-b), etzakicien (iho), etzakien (arü, ürr, sarr, gar, mend, mit), etzakitela (hoz), etzakiten (aiz, a-s, bid, buz, d-b, hel, iru, lekb, larz, lar, l-o, luk, zur, dono, dom, u-g, urk, z-p), etzakizien (alo, bar, bes, esk, gam, garr), etzakozien (d-b), etzaquicien (a-zo, g-zo), etzaquien (l-a), etzaquiten (arb, bir, hal, jat, bai), etzen (l-a), etzi (d-b), Etzi (garr), etziakizien (izp, jats), etzicien (iho), Etzicien (iho), etzien (ürr, gar, l-a, mit), Etzien (ürr, gar, l-a, mit), etzioten (hal, iru, jat), etziouten (a-s), etzitien (buz), etzitiin (hel), etzitin (bid), etzititzien (alo), etzitizun (esk), etzituen (bir, jat), etzituin (hal), etzituzten (larz), etzitzien (bes, iho), Etzitzien (bes), etzitzin (izp, jats), etziusten (azka, dono), etziuzten (l-o, zur, dom), etzizien (alo, bar, bes, d-b, esk, gam, garr, garr, iho, izp, izp, jats, urk), Etzizien (alo, bar, d-b, esk, gam, izp, jats), etzizun (gam), etzou (gam), etzouten (aha, arh, a-s, buz, hel), Etzouten (a-s, buz), etzu (hoz, izp), (etzuen) (luk), etzuten (aiz, aiz, arb, arr, azka, bid, bir, hal, iru, jat, lan, larz, l-o, luk, zur, bai, dom, u-g), (etzuten) (luk), Etzuten (aiz, arb, arr, azka, bid, hel, hal, iru, jat, lan, larz, l-o, zur, dono, bai, dom), etzutian (l-a), etzutien (ürr, gar, mit), etzutin (sarr, ünd), etzuzun (bar), ex (azk), Ex (azk), exakiten (d-g), exdute (lar), ez (a-m, aha, aint, arn, beho, eih, zar, ahe, ahe, ahe, ahe, ahe, ahe, ahe, ainh, alo, ame, ame, ame, ame, ame, amo, amo, 
amo, amo, anh, anh, anh, anh, anh, anh, anh, a-z, a-z, a-z, arb, arb, arb, arb, a-zo, arr, arr, arr, arr, arh, arh, arh, arh, arh, arh, arm, arm, arm, arm, arm, arm, arü, a-s, a-s, azk, azk, azk, azk, azk, azk, azka, azka, azka, azka, azka, altz, aih, aih, aih, aih, aih, aih, aih, beh, beh, beh, beh, beh, b-l, b-l, b-l, b-l, b-l, b-l, ban, ban, ban, ban, ban, ban, ban, bar, bard, bard, bard, bas, bas, bas, bas, bas, bas, bas, be-l, be-l, be-l, be-l, bid, bid, bid, bid, bida, bida, bida, bida, bir, bir, bir, lek, lek, lek, lek, lek, lek, lek, bes, bun, d-s, d-s, d-s, d-s, d-s, d-s, buz, buz, buz, g-z, g-zo, soh, soh, sarr, zib, zib, zib, zib, zib, zib, zib, zib, d-b, ünd, esp, esp, esp, esp, esp, esk, ezt, ezt, ezt, ezt, ezt, ezt, ezt, etx, etxa, gab, gab, gab, gab, gab, gab, gab, gam, gar, gar, garr, g-i, get, get, get, get, hel, hel, hal, haz, haz, haz, haz, haz, hau, hen, hen, hen, hen, hen, hen, hoz, hoz, hoz, iba, iba, ida, iho, iho, iho, iho, ilh, ilh, iri, iri, iri, iri, iri, iri, iru, iru, iru, izp, izp, izt, izt, izt, izt, its, its, its, its, its, its, its, jat, jats, jats, jut, jut, jut, jut, jut, jut, l-b, l-b, l-b, lak, lak, lak, lak, lak, lak, lak, laka, lekb, lekb, lig, lah, lah, lah, lah, lah, lah, lah, lan, lan, lan, larz, larz, larz, larz, larr, lar, lar, l-s, las, ald, ald, ald, ald, ald, ald, ald, osp, osp, osp, osp, l-a, l-o, luh, luh, luh, luh, luh, luh, luh, luk, luk, luk, luk, mak, mak, mak, mak, mak, mak, mak, meh, meh, meh, meh, meh, meh, meh, leko, leko, leko, leko, leko, leko, men, men, mend, mend, mend, mend, mug, mug, müs, ora, ora, ora, urd, ost, o-z, ort, ort, ort, ort, ort, ort, izu, izu, izu, izu, pag, arro, s-g, d-l, d-l, d-l, d-l, d-l, d-l, d-l, d-g, d-g, d-i, d-i, d-i, d-i, d-i, d-i, sen, sen, sen, sen, don, hir, hir, hir, hir, hir, hir, sar, sar, sar, sar, sar, sar, sar, zal, zur, zur, zur, dono, dono, dono, dono, bai, bai, bai, bai, dona, dona, dona, dona, dona, dona, dona, dom, dom, dom, suh, suh, suh, suh, suh, suh, suh, ath, irur, irur, u-g, u-g, u-h, u-h, u-h, u-h, u-h, u-h, u-h, urk, ure, ure, ur-p, urr, urr, urr, urr, urr, urr, urr, uzt, uzt, uzt, uzt, uzt, uzt, mil, mil, mil, mil, mil, mil, mil, bil), Ez (ahe, amo, anh, arh, arm, arn, beho, b-l, bas, bida, d-s, ünd, esp, ezt, get, hen, iri, iri, izt, its, jut, lak, lekb, lah, ald, osp, luk, mak, meh, leko, mug, mug, ort, d-i, don, hir, sar, dona, suh, u-h, urr, uzt, mil), ezçakiten (b-l), ezçakizin (arm), ezakiten (ur-p), ezçuten (d-i), ezçutian (osp), ezbaïtu (mug), ezbeitu (dom), ezciakicien (iba), ezcitcien (iba), ezcituzten (get), ezciuzten (mug), ezcuten (mug), ezda (ame, osp, l-o), (ezda (luk), ezdéçaten (aint, a-z), ezdadin (osp), ezdao (hel), ezdeçaten (ure), ezdezaten (beho, bes, gam, garr, jats), ezdiena (garr), ezdienin (bes), ezdiinian (hel), ezdizu (bes), ezdou (a-s), ezdu (luk, mug, u-g), ezduenian (lar, zur), ezdutelaric (u-g), ezduzu (jats), ezitititzun (bar), eziuzte (urk), eziuzten (lan), ezpadu (ida), ezpeïtcien (altz), ezpeïtou (gam), ezpeitu (gar, s-g), ezpeitoute (a-s), ezpeitu (ürr, be-l, sarr, ünd, g-i, mit, müs, arro, zal), ezpitou (lekb), ezta (a-m, aint, zar, ürr, sarr, g-i, hau, mar, mit, müs, urd, zal), Ezta (lar), eztéçaten (zar), eztçaquicien (etxa), eztadin (bar, zal), eztîlaïc (bai), ezt'suten (ban), eztçuten (izt), eztcicien (etxa), Eztcicien (gab), eztcitcien (etxa), ezteçan (müs), ezteçaten (a-m, amo, g-zo, etxa, ilh, l-b, las), eztecen (g-i, laka, men, s-g), eztenian (hal, jat), eztezan (alo), eztezaten (aiz, d-b, izp, urk), eztezen (soh, esk, gar, mit, arro), eztianian (alo, etx, etxa, laka, l-z), eztiénian (zar), eztiçu (altz, men, s-g), eztienïn (urd), eztienian (aiz, buz, d-b), eztilaric (las), eztinin (bar, esk, urk), eztitzu (izp), eztizu (alo), eztu (aiz, hal, ilh, jat, las, d-g, hir), eztuçu (etxa, ida, laka, larr, l-z, arro, bil), eztuenian (bir), eztutelarik (d-g), eztuzu (bar, esk, urk), ezuten (ur-p), Ezuten (bir, ur-p), ezuzten (lekb), etçaquiten (aha), etzuzten (a-m), ezbaitou (eih), ezpaitu (a-m), etciousten (aha), etciouzten (aint), estienian (aha), eztinian (aint), eztçakiten (aint), esdeçaten (eih), eztanic (beho) 
ezagutu (145): arra_ezagoutourik (a-s), éçagoutia (don), éçagoutoua (ain, hen), éçagutouric (ito), éçagutu (alt, a-z), éçagutuic (hau), éçagutuik (etx, ath), éçaguturic (larr), éçagutuya (mug), éçaoutia (aint, zar, beh), éçaoutouic (iba), ésagoutia (kan), eçaütia (gab), eçaütuya (leko), eçagoutia (eih, jut, ora), Eçagoutia (bit, dona), eçagoutoua (arm, sen), Eçagoutouya (lah), eçagutcen (bida), eçagutia (a-m, anh, iru, lan, las, mak, mar, ort, pag, d-g, d-i, u-g, z-p), Eçagutia (ure), eçagutu (ainh, altz, esp, lar, urd, hir), eçagutua (b-l, bida, izt), Eçagutua (azk, its, urr), eçagutuic (l-z), Eçagutuik (men), eçaguturic (arü, g-z, etxa, g-i, ida, laka, osp, l-a, mau, müs, s-g, zal), Eçaguturic (amo, be-l, soh), eçaguturik (bil), eçaoutia (l-b), Eçaoutia (aha, ilh), eçaoutu (a-zo, g-zo), eçautia (mend, izu), ecaguturic (arro), esa’utia (ban), Esagoutia (ald), esagutia (l-o), Esaguturic (l-s), essagoutia (bun), Essagutuic (o-z), essaguturic (lig, irur), Essaguturik (mit), Essaoutia (bard, ost), ezaütia (bai), Ezaütia (aiz, izp), ezaütua (bas), ezaütuik (db), ezaûtuya (zur), ezagoutia (arh, buz, lekb), ezagoutua (hel), Ezagoutua (ame, meh), ezagutia (arn, beho, d-s, iho, jats, lak, larz, suh, u-h), Ezagutia (bid, luk, dom), ezagutu (esk, iri, d-l), Ezagutu (aih, uzt), ezagutua (ahe, arb, arr, zib, get, hal, jat, dono, mil), Ezagutua (haz, sar), ezaguturic (ünd), ezaguturik (alo, bar, sarr, gar), Ezaguturik (ürr), ezaoutia (azka, gam), ezaoutu (garr), Ezautua (bir, ur-p), ezauturik (urk), Ezautuya (bes)

ezarri (3): eçartio (mar), ezar (garr), ezari (esk)

ezdeustatu (1): esdestatu (hel)

ezen (13): -ecen (iri, its), ésen (kan, kan), ecen (hal, jat, ora), -ecen (b-l, uzt), Ecen (bida), ezen (sar), (ezen (hal, jat)

ezeztatu (22): ézeztatu (beh), ecestatu (mend), eceztatou (dona), eceztatu (gab), eceztatzen (iri), esestatu (bir, haz), essestatuziren (aiz), etsestatu (mil), ez estatu (ure), ezestatu (lek, iru, jats), ezestatzen (azka), ezetatu (arb), ezeztatou (buz), ezeztatu (ahe, arr, aih, izp, larz, suh)

ezi (3): eci (müs, urd), ezi (bar)

ezik (5): -ezik (zib), ecic (hoz, arro, arro), ezik (d-l)

ezin (3): écin (ath), ecin (iru), ezin (ahe)

faltatu (1): faltatu (beho)

feit (1): Feit (altz)

fite (77): fité (aint, zar, alt, bit, bun, kan, g-z, iba, ilh, izt, l-b, mau, ost, don, z-p), fite (aha, alo, a-m, ame, amo, arh, arm, arü, a-s, azka, ban, bard, beh, beho, be-l, bid, d-s, buz, eih, soh, sarr, d-b, ünd, ezt, gab, gam, hel, haz, iru, izp, its, jats, jut, lak, lekb, lig, lah, lan, luh, mak, leko, men, mend, müs, ora, izu, d-g, zal, zur, dono, dona, suh, irur, u-g, u-h, urk, mil, bil), fitechko (dom), fitez (b-l, larr)

fitsik (3): fitsic (d-l), phitsic (lek, haz)

franko (13): frango (aha, a-m, d-s, hen, izt, d-g, bai, u-g), frangotan (arh, d-g, u-g, u-h), franko (ur-p)

frantses (1): frantsesez (d-b)

frontera (3): fonteraco (iho), fonteraco (Pyrénées) (iho), funteraco (lan)

fruitu (129): frouïtouënn (hen), frouïtouen (kan), frouitién (don), frouitiènn (bit), frouitien (aha, aint, jut, dona), frouitou (bun), frouitouen (lah, sen), frouituen (hel), froutén (ost), frouten (a-s, lekb), froutien (zar, buz, gam), fruituen (gab), fruiten (ame, jats, luk), fruitién (las), fruitien (amo, anh, arn, azka, aih, beh, ban, d-b, eih, ezt, larz, 
mend, ora, d-g, bai, dom, u-g), fruitier (izp), fruitiren (bil), fruitouen (arm, iba, ald), fruitouentzat (luh), fruituen (ahe, azk, beho, b-l, bir, lek, d-s, zib, get, haz, hoz, iri, iru, izt, its, lan, mak, meh, leko, ort, d-l, d-i, hir, dono, suh, ure, ur-p, urr, uzt), fruituien (urk), fruituyen (bes, mug, zur), frutën (g-z), frutén (l-s), fruten (aiz, ainh, alt, a-m, arh, arü, altz, be-l, soh, sarr, esk, etxa, gar, ilh, l-b, lig, l-a, izu, pag, s-g, ath), frutien (a-z, a-zo, bard, g-zo, etx, g-i, hau, ida, iho, ito, lak, laka, larr, l-z, mar, mau, men, mit, müs, o-z, z-p), frutin (ünd, u-h), frutuen (bas, mil), fuituen (bid)

funts (2): fountsian (a-zo), fountxian (g-zo)

furia (1): furian (las)

furios (7): fouriojés (ost), fourios (buz), fouriosés (hen), fouriosez (dona), furiüsez (luk), furiosen (iho), furius (mil)

fuxos (4): fuïouzés (hau), fuchos (jats, suh), fuchosez (jut)

gabe (158): batere_gabe (osp), bateren (mar), Galdingabe (gam), gaa (hel), gabé (ain, zar, ainh, a-z, a-zo, altz, beh, bit, bun, bun, kan, hau, hen, iba, ito, lah, l-s, mug, ost, don, dona, ath, ur-p, z-p), gabétaïc (g-z), gabe (aha, ahe, aint, alo, a-m, ame, amo, anh, arb, arn, arr, arh, arm, arü, a-s, azk, azka, aih, ban, bar, bar, bard, bas, beho, b-l, be-l, bid, bida, bir, lek, lek, bes, d-s, buz, eih, g-zo, soh, sarr, zib, d-b, ünd, esp, esk, ezt, etx, etxa, gab, gar, garr, g-i, get, hal, haz, hoz, ida, iho, ilh, iri, iru, izp, izt, its, jat, jats, jut, l-b, lak, laka, lan, lan, larz, larr, lar, lar, las, ald, osp, l-z, l-a, l-o, luh, luk, mak, mar, mau, meh, leko, men, mend, müs, ora, urd, o-z, izu, pag, arro, s-g, d-l, d-g, d-i, sen, hir, sar, zal, zur, dono, bai, dom, suh, irur, u-g, u-h, urk, ure, urr, uzt, mil, bil), gabea) (bida), gaben (g-i), gabetaric (ürr, lekb, lig, ort), gabetarikk (mit), gabez (ida), gabiac (d-s)

gain (209): gaïn (urd), gañan (d-b, d-b), gañen (ürr, ürr, bar, bar, esk), gaïgnian (bun, bun), gaïn (ahe), gaïnéan (ain, ain), gainéann (hen, hen), gainean (arb, arm, haz, iru, mug, sen, sen, ure), gaïneco (ure), gainen (zar), gainian (aha, aint, aiz, a-m, anh, arb, arr, buz, eih, hel, hel, ito, jats, jats, lah, las, las, luk, meh, mend, ora, don, bai, bai, dona), gaïnian (a-m), gaïntilat (gar), gagnan (a-z, a-zo, gab, gab), gagnën (g-z), gagnén (hau, hau), gagnen (ainh, alo, amo, arü, bard, be-l, ünd, etx, etxa, gar, gar, g-i, g-i, ida, laka, lig, larr, l-z, mau, men, men, mit, mit, müs, müs, o-z, pag, pag, arro, s-g, ath, ath, irur, bil), gagnian (a-s, g-zo, ezt, gam, gam, iba, ida, ilh, ilh, l-b, l-b, larz, l-s, l-a, l-o, ora), gagniane (ost, ost), gagniann (bit), gaiñ (arro), gaiñean (zib, zib), gaiñen (osp), gaignen (soh), gaignian (jut, jut, jut, jut), gain (bida), gainean (ahe, ame, ame, arm, arn, arr, azk, b-l, bida, get, get, iri, iri, iru, izt, its, its, lar, mak, mak, mar, leko, leko, ort, ort, d-l, d-l, d-g, hir, sar, sar, dono, suh, u-g, urr, uzt, uzt, mil, mil), gaineko (urk), gainen (z-p), gainian (anh, azka, aih, beh, beh, beho, ban, bas, bid, bir, lek, lek, d-s, esp, esp, hal, hal, hoz, iho, iho, izp, izp, jat, jat, lan, lan, ald, meh, mend, zur, dono, dona, urr), gainin (bes, bes, urk, urk), gaintica) (z-p), ganat (sen), ganian (lekb), gaynean (a-s), gaynia (ur-p)

gaitz (33): gaïtça (aint, zar), gaïtca (don), gaïtcac (aint), gaitcak (zar), gaïtcez (amo, arü, eih, ünd), gaïts (g-z), gaïtz (alt, bit, mau, müs, bil), gaïtzez (alo, gar, lekb), gaitcez (sarr, d-i), gaitz (bar, bar, be-l, soh, esk, g-i, iba, larr, l-o), gaitza (iho, lan), gaitzac (uzt), gaitzez (men)

gaixo (1): gaichuec (iho)

gaizki (2): gaïski (altz, altz)

gaizto (178): gaïchto (alo, altz, g-z, sarr, etxa, etxa, garr, l-s, s-g, don, irur), gaïchtoa (lar), 
gaïchtoac (anh, arb, luk, meh, sen, bai), gaïchtoez (sen), gaïchtouac (aha, arm, hel, iba, ito, ora, dona), gaïchtouak (buz), gaïchtouez (ora), gaïchtuac (arn), gaïstho (aiz), gaïsto (zar, alt, ida, lig, o-z, pag), gaîsto (soh), gaïstoac (ain, mug), gaïstoak (ahe), gaïstouac (arh, eih, hen), gaïstouak (bit), gaïstouez (urd), gaïstuac (d-g, u-h), gaïstutik (ath), gaïztiez (etx, l-z), gaïzto (aiz, müs), gachto (a-m, a-z, a-z, esk, etx, gar, hau, laka, lekb, lar, l-z, men, ure), gachtoac (haz, its, lah, ald), gachtoak (leko), gachtos (aha, ban), gachtotçu (ost), gachtouac (amo, bun, kan, gab, mend), gachtouak (azka, beh, 1-b), gachtouaktzou (gam), gachtouès (bun, kan), gachtouez (a-s), gachtuac (aih, ban, jats, izu, dom), gaichto (aint, arü, bar, beho, be-l, bes, d-s, d-b, ünd, esp, larz, osp, l-a, arro, hir, zal, ath), gaichtoac (ame, b-l, bid, get, hal, iru, izt, jat, lan, ort, d-i, hir, dono, uzt), gaichtoak (arr, esp, mil), gaichtoès (izt), gaichtobatzuz (azka, d-s), gaichtoez (get, leko), gaichtouac (g-zo, jut, osp), gaichtouactçu (a-zo), gaichtouak (ilh), gaichtuac (bas, hoz, iho, izp, las, suh), gaichtuk (urk), gaisto (ainh, arb, arr, ürr, g-i, larr, l-o, mau, mit, urd), gaistoac (azk, ezt, mak), gaistoak (ezt, d-l), gaistouac- (mar), gaistoues (ida), gaistuac (bir), gaistuak (lek, u-g), gaistues (d-g), gaistuez (u-g), gaisturic (l-a), gaizto (z-p), gaiztoac (urr), gaiztoak (sar), gaiztoez (sar, urr, z-p), gaizturik (laka), gastoac (lak), gastuak (ur-p), gaychto (a-s, bil), gueistouak (bard)

gaiztu (1): gaïstiac (o-z)

galant (1): Galant (arro)

galde egin (7): galde egin (d-g, meh), Galde eguin (a-m, arh, dono, ezt), Galde in (g-zo)

galdegin (87): galdéguin (a-z, lah), Galdéguin (bun, kan, l-s), Galdéin (aint, zar, ito), galde’in (ban), galdeïn (gab), Galdeïn (urk), galdegin (arn, bid, buz, hoz, lak, u-g), Galdegin (aih, beho, d-s, iho, l-o, men, sar, zur, u-h, mil), Galdeginen (lak), Galdegiten (lak), galdeguin (amo, anh, arm, a-s, b-l, etxa, iru, izp, lar, las, mar, mug, ora), Galdeguin (ahe, ame, eih, hal, iri, izt, its, jat, jats, lekb, lan, ald, luk, ort, d-i, dona, dom, suh, ure), galdeguiteco (anh, izt), galdein (bit, hel), Galdein (beh, bes, d-b, leko, mend, bai), Galdiin (larz), galdin (aha, garr, ilh, jut, l-b), Galdin (azka, iba, izu), galdine (ost), Galdingabe (gam), galthegin (altz), galtheguin (gar, müs, pag)

galdetu (56): Galda (a-zo), galdatou (luh), Galdétou (ain, hen, don), galdatu (lek, haz), Galdatu (arb, arr, bard, bas, bir, esp, mak, uzt), galdetou (sen), galdetu (zib, hir, ur-p, urr), Galdetu (azk, bida, get, d-l), galthatu (alt, alo, ürr, g-i, hau, ida, laka, mit, o-z, s-g, bil, z-p), Galthatu (ainh, arü, be-l, g-z, soh, sarr, ünd, esk, etx, lig, larr, osp, l-z, l-a, mau, urd, arro, zal, ath, irur)

galdu (68): galcen (urd), galdou (arh, kan, jut, lekb, ald, ora), galdouçirènn (bit), galdu (alt, alo, amo, a-z, a-zo, arü, ürr, altz, b-l, ban, bar, be-l, g-z, g-zo, soh, sarr, ünd, esk, etx, etxa, gar, g-i, hau, ida, iho, ilh, l-b, laka, lig, larr, l-s, osp, l-z, l-a, mak, mau, men, mit, mug, müs, izu, arro, s-g, d-l, d-i, zal, dono, dom, ath, irur, u-h, urk, bil, z-p), galtcen (izt, lar), galtciagatic (ida), galtzen (l-o), galzen (lan)

gardazale (1): gardazalé (ur-p)

garrasi (1): garassia (anh)

garratz (1): garatsac (kan)

gastigu (2): gaztiguia (etx, l-z)

-gatik (19): gatic (aha, ain, amo, anh, a-z, bar, bas, eih, ezt, its, jut, l-a, luk, ost, ort, d-i, dona), içanagatic (iri, ), issanagatic (zur)

gau (7): gaï (altz), Gaü (zib), gaüak (garr), Gaiaren (ida), Gaoua (bard), Gauan (mar), Gaubaren 
(urr)

gaur (9): gaöur (hen), Gaoür (hen), gaurco (hal, jat), Gaurco (hal, jat), gaurko (zib), Gaurko (zib, d-l)

gauza (147): Bergaïça (bil), gaüça (arm, iru, ort), Gaüça (d-g, ure), gaüca (anh), gaücez (mug), gaüsa (luk), gaüssa (bun), gaüza (d-b, garr, haz, jats, meh, leko, u-h), Gaüza (bai), gaïça (arü, be-l, hau, osp, müs, pag, ath, bil), Gaïça (altz, g-z), gaïca (o-z, s-g), gaïssa (irur), gaïssetaïc (irur), gaïssetaric (lig), gaïza (alo, sarr, ünd, gar), gaûzen (lek), gaiça (ainh, soh, etxa, g-i, laka, larr, l-a, mau, men, arro, zal, z-p), Gaiça (ürr, mit), gaiçac (etxa), gaiz (ame), gaiza (bar), Gaiza (esk), Gaoüça (hen), gaoüza (gam), gaouça (aha, ain, aint, a-zo, beh, bard, bit, g-zo, iba, ilh, ito, jut, l-b, lah, ora, dona), Gaouça (sen, don), gaousa (arh, kan, l-s), gaousetaric (ald), gaoussa (ost), gaouza (hel), gauça (a-m, eih, zar, amo, a-z, azk, b-l, bida, esp, esp, izt, its, lar, las, mak, mar, izu, d-i, hir, hir, dom, urr), gauças (gab), gaucen (esp), gausa (arb, ban, hoz, l-o, luh, ur-p), gautsa (ald, mend), gauza (ame, arn, arr, a-s, azka, bas, beho, bid, bir, bes, buz, ezt, get, hal, iho, iri, izp, jat, lak, lekb, larz, d-l, sar, zur, dono, suh, urk, uzt, mil), Gauza (aih, u-g), gauzak (a-s)

gaztaina (147): castagna (bir), gaistagna (be-l, soh), gaiztaignac (get), gaiztaina (hir), gastaña (d-b, ünd, hoz), gastaïgna (luk), gastaïna (aiz, arb, arr, kan), gastagna (aha, ainh, alt, amo, a-zo, arü, altz, bit, hau, hen, ito, larr, l-s, l-a, mar, mau, ora, ost, o-z, izu, d-g, ath, z-p), gastégna (g-z), gastagnac (bun), gastaignac (ald), gastain (azk), gastaina (ame, mak, d-l, u-g), gastan (ur-p), gastana (d-i), gastegna (mit, u-h), gasteigna (etxa), gastena (b-l, ban, eih, esp, its, jat, lar, luh, mend, zur, mil), gastigna (ürr), gaztaña (am, bar, sarr, zib, esk), gaztaïgna (jut), gaztaïn (sen), gaztaïna (ain, iru, lekb, las, meh, don), gaztagna (alo, a-z, azka, bard, beho, buz, g-zo, ezt, etx, gab, gam, gar, g-i, iba, ida, ilh, izp, jats, l-b, laka, lig, lan, larz, l-z, l-o, müs, pag, arro, s-g, zal, irur), gaztagnac (urd), gaztagnak (bida), gaztagnaz (garr), gaztaiña (osp), gaztaina (ahe, beh, d-s, iho, lak, sar, dona, urr), gaztainac (iri, dono), gazténa (zar, lah), gazténac (izt), gazteïnac (bas), gaztegna (arh, men, bil), gazten (bid), gaztena (aint, anh, arn, lek, bes, hel, hal, haz, leko, mug, ort, bai, dom, suh, ure, uzt), gaztenac (aih), gaztenak (urk), gazteyna (a-s), gaztina (arm)

gehiago (8): gehiago (d-s, zib, urk), gehio (d-g, u-g), guehia'o (ban), guehiago (a-zo, g-zo)

geldi (23): geldi (hel, hoz, lak), geldi-arazten (sar), geldirik (d-s), guéldi (hen), gueldi (anh, aint, a-z, a-zo, beh, eih, bid, g-zo, gab, mak, mug, suh, ur-p, zar), gueldibat (a-s, mar), gueldic (izu)

gelditu (70): gélditu (ito), gelditu (bes, haz, hoz, iho, iri, lak, l-o, luk, meh, sar, zur, urk), guélditou (ain, bit, kan, iba), guelditceco (b-l), guelditou (aint, zar, arh, arm, hel, hen, lah, ald, ora, sen, don), guelditu (aiz, ahe, a-m, ame, anh, arb, arr, azk, aih, beh, b-l, ban, bard, bas, bid, bida, bir, eih, lek, esp, ezt, get, iru, izt, las, mak, leko, mug, ort, d-i, hir, dono, bai, dom, suh, u-h, ure, ur-p, urr, uzt), guelditzen (a-s)

gero (283): géro (ito), geo (bes, bes, d-b, d-b), gero (arn, beho, ürr, ürr, bar, bar, bar, bid, d-s, d-s, buz, buz, sarr, zib, ünd, esk, esk, haz, haz, hoz, hoz, hoz, lak, lak, l-o, l-o, luh, luk, meh, meh, men, men, mit, mit, d-l, d-l, d-g, d-g, sar, sar, zur, zur, u-g, u-g, urk), Gero (bida), geuro (bas), gio (l-b, l-b), guïo (gam), guéo (bit, g-z, etx, etx, etx, hau, hau, iba, iba, ost, o-z, ath), guèro (bun, bun), guéro (ain, ain, aint, zar, zar, ainh, alt, a-z, a-z, beh, beh, bit, bit, kan, kan, hen, hen, izt, lah, lah, l-s, l-s, z-p), Guéro (don), 
gueo (bard, gab, gab, hel, hel, l-z, l-z, l-z, leko, leko, o-z, izu, pag, pag, ath), Gueo (izu), guero (aha, ahe, ainh, ainh, alo, alo, a-m, ame, ame, amo, amo, anh, anh, arb, a-zo, a-zo, arr, arh, arh, arm, arü, arü, arü, a-s, a-s, azk, azk, azka, azka, aih, aih, b-l, ban, bard, bas, bas, be-1, be-l, bid, bir, bir, d-s, eih, g-zo, g-zo, soh, soh, sarr, ünd, ünd, esp, esp, ezt, ezt, etxa, etxa, gar, gar, gar, g-i, g-i, get, get, hal, hal, ida, ida, iri, iri, iru, iru, izp, izp, izt, its, its, jat, jat, jats, jats, jut, jut, laka, laka, lekb, lekb, lan, lan, larz, larz, larr, larr, larr, las, las, ald, osp, osp, l-a, l-a, luk, mak, mak, mar, mar, mau, mau, mend, mend, mug, mug, müs, müs, ora, ora, urd, urd, ort, ort, arro, arro, s-g, s-g, d-i, d-i, sen, sen, hir, zal, zal, dono, dono, bai, bai, dona, dona, dona, dom, dom, suh, suh, u-h, u-h, ure, ure, ur-p, ur-p, urr, urr, uzt, uzt, mil, mil, bil, bil, z-p), Guero (aih, garr, lig, ald, irur), guio (arm, ilh, ilh), Guio (gam)

gertakari (5): gerthacaria (hoz, hoz), Guerthacaria (b-l), guerthakaria (bard, bard)

gertatu (36): gertatu (haz, d-g, u-g), gertatzen (urk), gerthatcen (d-g, u-g), gerthatu (arn, bida, d-s, iho, lak, d-l), guérthatcén (ost), guérthatou (ain, ain, bit), guérthatsèn (bit), guertathu (aiz), guertatu (ban), guertatzen (aiz), guerthatcen (a-z, lek, bun), guerthatou (arm, bun, hel, ora), guerthatu (azk, ilh, izp, lar, luk, bai), guerthatucen (hir), guerthatuzen (aih), guerthatzen (azka)

gibel (6): guibel (l-a), guibelat (hal, jat), guibeletic (hel, iru), guibeletik (ahe)

gibelatu (8): guibelarazten (iru), guibelatcen (hal, jat, sen), guibelatcentu (esp), guibeltcen (lz), guibeltsen (etx), guielatcen (gab)

gisa (40): bergijan (men), bergisan (mit), Berguisan (etx, gam, z-p), gisa (beho, lak, luh), Gisa (buz, u-h), gisan (arh), Giza (arh), gizan (u-h), guiçann (hen), guichan (sen), Guija (iba), guijan (aha), Guijsa (ban), guisa (ora, dona), Guisa (kan, iho, iru, jats, arro, dono, bai), guisan (a-s, bid, etxa, iru, laka, lekb, mau, müs, izu), Guise (bida), Guissa (a-z), guizan (etxa, gar)

gisa berean (2): guisa_berean (ure), guisaberian (mar)

gizon (414): guiçoun (etx), giçon (a-m, l-b, l-b, men, men, men, d-g, d-g, zal), giçoun (ito, l-b), gison (luh, luh, luh, luh, luh, luh, mend, mit), Gison (luh), gizün (d-b, d-b), gizon (arn, beho, ürr, ürr, lek, lek, lek, lek, bes, bes, bes, d-s, d-s, d-s, buz, buz, buz, sarr, sarr, sarr, zib, ünd, ünd, ünd, get, haz, haz, haz, haz, haz, hoz, hoz, hoz, iho, lak, lak, lekb, lekb, lan, l-o, l-o, l-o, meh, meh, mit, mit, d-l, sar, sar, sar, sar, zur, zur, u-g, u-g, u-g, u-h, u-h, u-h, urk, urk, urk, mil), gizona (d-l, urk), gizoun (bar, bar, bar, esk, esk, esk), gizun (d-b), guiçon (aha, ain, ain, ain, a-m, zar, zar, zar, ainh, ainh, ainh, alt, alt, amo, anh, anh, a-z, a-z, a-z, a-zo, arm, arü, arü, arü, azk, azk, altz, altz, altz, beh, beh, b-l, b-l, b-l, b-l, b-l, bida, bida, bida, bun, bun, eih, g-z, g-z, g-z, esp, esp, esp, etxa, etxa, etxa, g-i, g-i, g-i, hel, iba, iba, iba, ida, ida, ida, ilh, ilh, iru, iru, iru, izt, izt, izt, its, its, its, jut, laka, laka, laka, lah, lah, lah, larr, larr, larr, lar, las, las, ald, mak, mak, mak, mar, mar, mau, mug, mug, müs, müs, ora, ora, ora, urd, urd, urd, o-z, o-z, ort, ort, izu, izu, pag, pag, pag, d-i, d-i, sen, sen, don, don, don, hir, hir, hir, dona, dona, irur, irur, ure, ure, ure, urr, urr, bil, bil, bil, z-p, z-p), Guiçon (altz, urd), guiçona (bit, lah), guiçonbat (mar, müs), guiçonec (arm), guiçonik (don), guiçonn (a-m, bit, bit, hen), guiçoun (amo, amo, a-zo, bard, bard, be-l, g-zo, g-zo, g-zo, soh, soh, etx, hau, hau, ilh, lig, osp, osp, osp, l-z, l-z, l-a, l-a, l-a, arro, arro, arro, arro, s-g, s-g, s-g, zal, zal, ath, ath), Guiçoun (osp), guiçounbat (a-zo), guiçun (gab, gab, gab, hau, mau), guicoun (etx, mau), guisom (ban), guison (aint, alt, arh, arh, ban, ban, ban, kan, kan, 
kan, ald, ald, luk, mend, mend), guisson (hen, lig, ost), guissone (ost, ost), guissounn (bit), guizon (aiz, aiz, aiz, ahe, ahe, ahe, ahe, ahe, ame, ame, ame, arb, arb, arr, arr, arh, arm, ürr, a-s, a-s, a-s, azka, azka, azka, aih, aih, aih, bas, bas, bid, bid, bir, bir, ezt, ezt, ezt, gam, gam, garr, get, get, hel, hal, hal, hal, iri, iri, iri, izp, izp, izp, jat, jat, jat, jats, jats, jut, lekb, lan, larz, larz, larz, l-s, l-s, l-s, l-s, luk, leko, leko, leko, dono, dono, bai, bai, bai, dom, dom, dom, suh, suh, suh, ur-p, ur-p, ur-p, uzt, uzt, mil), Guizon (aih), guizona (ahe), guizoun (alo, alo, alo, be-l, gar, gar)

goait (7): gouaita (lig, irur), gouaitian (arh), gouaitiann (beh), goueïtian (a-zo), goueytian (gzo), gouitan (bar)

gogo eman (16): go émak (beh), Gogo eman (hoz), gogo emac (ora), Gogoemac (bit), Gogouman (irur), Gogua (be-l, be-l, soh, soh), gomac (a-zo), Gomac (a-z), gomak (garr), Goman (ort), gomaneçan (a-zo), Gooemac (g-zo), gooemaneçan) (g-zo)

gogor (2): gobor (aiz), gohorrekoak (bida)

gogorki (2): gogorki (d-g), gohorki (u-g)

goiz (138): goïçan (arü, ida, pag, arro, s-g, ath), goïçann (hau), goïçiann (bit), goïcéan (ain, mug), goïcéänn (hen), goïcan (müs), goïcean (meh, ure), goïcian (a-m, aha, aint, zar, amo, a-z, a-zo, arh, beh, be-l, g-z, g-zo, etxa, gab, iba, ito, l-b, lah, l-s, las, ora, d-g, don), goïciane (ost), goïcien (bard), goïsian (kan, luk), goïssian (bun, irur), goïzan (sarr, ünd, gar), goïzean (arb, arr), goïzian (alo, arm, buz, gam, hel, haz, jats, lekb, bai, u-h), gocian (ur-p), goiçan (ainh, laka, larr, osp, men, urd), goician (bir), goicean (arn, bida, iru, izt, its, leko, ort, d-i, sen, hir, uzt), goician (anh, azk, bid, eih, esp, ezt, g-i, hoz, ilh, jut, l-a, mak, mar, mau, mend, izu, zal, dona, suh, u-g, urr), goisean (luh), goisian (ban, ald, l-o), goissan (o-z), goissian (lig), goitzian (lan), goizian (lek), goizan (ürr, bar, esk, mit), goizean (ahe, b-l, zib, iri, lak, d-l, sar, mil), goizian (ame, azka, aih, bas, beho, d-b, hal, iho, izp, jat, larz, zur, dono, dom), goizin (bes, urk), goyçan (bil), goycian (z-p), goyzian (a-s)

gorde (102): gordatcën (g-z), gordatcén (hau, iba, ost, don), gordatcen (a-m, aha, aint, zar, ainh, alt, amo, a-zo, arm, arü, beh, be-l, g-zo, etx, etxa, g-i, ida, iho, ilh, izt, jut, l-b, laka, larr, ops, l-z, mar, mau, men, müs, ora, urd, izu, pag, arro, s-g, zal, suh, ath, bil, z-p), gordatsènn (bit), gordatsen (bun, kan, lig, l-s. o-z, irur), gordatzen (alo, ame, arh, ürr, a-s, bar, d-s, buz, sarr, d-b, ünd, esk, ezt, gam, gar, hel, hoz, izp, jats, lak, lekb, lan, larz, l-o, luk, mit, dom, u-h, urk), gordé, (aint, zar, o-z, don)gordëic (etx, l-z), gordéic (o-z), gordéik (ath), gordétcén (ain),gordétzen (lah), gorde (arm, bid, bida, pag) gordeïc (g-z, hau) gordeïk (laka), gordecen (bir, ur, p), gordeic (arü, Und), gordeik (sarr, men), gorderic (ainh, ürr, esk, etxa, g-i, iru, larr, lar, ald, osp, l-a, mau, mit, urd, s-g, bil), gorderik (altz, bar) gordetcénn (hen), gordetcen (anh, arn, a-z, arb, arr, azk, b-l, bard, bida, eih, soh, esp, gab, get, its, las, mak, meh, leko, mug, ort, d-i, sen, dono, dona, u-g, ure, urr, uzt), gordet-cen (hir), gordetsen (luh, sar), gordetzen (ahe, azka, aih, ban, bas, beho, bid, lek, bes, zib, hal, haz, iri, jat, mend, d-l, zur, bai, mil), gordez (l-a), gordia (lekb, mend, pag, don, z-p)

d-g, sar, zal, zur, dono, ath, u-g, urk, uzt, bil), goseac (arn, b-l, its), goseak (leko, hir), gosia (altz), gosiac (aih, a-m, hoz, iri, mend, bai, suh, urr), gosiak (azka, buz, lak, d-l), gosjé (iba), gossé (ain, aint, zar, a-zo, beh, bit, eih, g-zo, ilh, l-b, lig, lah, l-s, o-z, irur), gosse (ainh, anh, arü, bard, be-l, soh, etxa, gab, g-i, ida, larr, lar, osp, mar, mau, mit, müs, ora, urd, pag, arro, s-g), gosseac (ure), gossia (bun), gossiac (z-p), gossiak (gam), 
gotze (mil), goze (aiz, garr), gozetiak (ame)

gosetu (10): gosetiac (amo, arh, dom, u-h), gosetuac (d-i), gosetuak (ahe), gosetuik (laka), goshétuiric (alt), gossetiac (lan)

$\mathrm{gu}(1)$ : gu (osp)

guardatu (4): Gardiatzen (bas), goardiatsen (bun), Guardiatzen (jast, mil) guardatzaile (1): guardiatzalerik (mil)

guardia (49): Güardia (iru), gardariric (get), gardia (hen), Gardia (azk, bas), gardianoric (ald), gardienik (urk), Gaurdia (mil), goardia (anh, don), Goardia (ahe, anh, luk), goardian (anh, luk), gouardano (gam), Gouardi (a-s), Gouardia (kan, ilh, lah), gouardian (lah), Gourdia (arh) , guarda (aiz), Guarda (iho, iri), guardan (aiz), Guarde (suh), Guardi (bir, mak), guardia (aih, beho, jats, lak), Guardia (aih, b-l, izp, izt, lak, las, d-i, u-h), guardian (ame, b-l, ban, izt, larz, d-i, u-h), guardiariric (izt)

guardia eman (15): Gouardémac (iba), Gouardiemak (l-b), Gouardiemac (azka, ost, dona), Gouardimak (gam9, Guardaman (lan), Guardiac (ame), Guardi'emac (ban), Guardi'eman (ban), Guardiemac (utz), Guardimac (larz, d-g, dono, u-g)

guardiano (2): gouardianoric (buz), guardinoric (d-i)

gune (3): gune (l-a, arro, zal)

gunecal (1): günecal (alt)

gure (135): goué (ath), goue (bard), Goue (hel, hau), Goue-Jaouna (l-z), Goue-Jauna (etx), gouré (g-z, hen, dona, z-p), Gouré (ain, zar, bit, bun, mau, don), goure (aha, a-zo, arh, bar, be-l, lek, g-zo, g-i, lekb, lig, larr, ald, ald, l-o, luh, arro, arro, s-g, zal, irur, bil), Goure (ainh, alo, a-z, arü, ürr, a-s, ünd, gam, jut, l-b, laka, mar, men, pag, arro, sen, zal), Goure- (ida), Goure-Jaüna (sarr, mit), Goure-Jaouna (gar, o-z), GoureYauna (buz), Goure-Yincoa (arm), Guré (iba), gure (ahe, ahe, ame, ame, anh, arn, beh, b-l, ban, bas, bid, bida, d-s, d-s, esp, esp, esp, iri, izp, lar, luk, mak, mug, izu, d-l, hir, hir, sar, dono, dom, u-h, urr), Gure (ahe, aint, ame, azk, azka, beho, bir, soh, zib, ezt, get, hal, iho, ilh, iru, its, jat, jats, lak, osp, meh, leko, mend, ost, ort, zur, urk, ure, ur-p, mil), Gure-Jauna (hir), Gure-Jauna- (hir), Gure-Jincoua- (amo), guren (ida), GureSalbazailia (larz), Gure-Yaüna (gab, bai), Gure-Yauna (uzt), Gure-Yauna (a-m)

gustu (1): goustouac (luh)

guti (1): gutiago (z-p)

gutxi (1): guchihago (uzt)

guzi (258): gouçiac (bit, hen), goucia (aha, arh, hel, hen, lah, ora, don, dona), gouciac (aha, ain, ain, zar, arh, arm, arm, arm, eih, iba, jut, jut, lah, lah, ald, ora, ora, ort, sen, sen, dona, dona), gouciak (don), gouciétaric (ain, hen), Gouciétarik (don), goucietaric (ald, sen), gouciz (sen), gousia (kan, kan), gousiac (kan, kan), gousiétaric (kan), goussiac (bun), goussièc (bun), gouzia (a-s, buz, gam), gouziac (hel, lekb, lekb, lekb), gouziak (a-s, buz, gam, gam), Gouzietaric (lekb), guçiac (izt, ort), guçiak (beh), gucia (a-m, amo, azk, beho, bir, esp, gab, iri, iru, izt, izt, its, its, leko, ost, izu, dono, u-h, ure, uzt), guciac (a-m, ame, amo, anh, anh, arn, a-z, a-zo, azk, azk, beho, b-l, b-l, bid, bid, bir, eih, lek, g-zo, get, get, hoz, iho, iri, iru, iru, its, its, larz, lar, las, las, mak, mak, mar, mar, mar, meh, meh, meh, leko, mend, mug, mug, ort, izu, izu, d-g, d-i, d-i, hir, suh, u-g, u-g, u-h, ure, ur-p, urr, urr, urr, uzt), guciak (arb, arr, bida, bida, esp, ilh, l-b, d-g, urp), gucian (arn), guciétaïc (a-z), gucien (lar), Gucietaic (iba), gucietaic (bir), gucietaik (bida), gucietaric (beho, b-l, bid, iho, iri, izt, its, meh, d-i, hir, urr), Gucietaric (azk, 
iri), gucietarik (haz), Gucietarik (esp), guciez (lek), gucitaco (d-g, u-g), gucitaic (izu), gusiac (ban, ban, l-s), gusiak (bas, l-o), Gusieta'ic (ban), gussiac (ost), guzia (aiz, ahe, ahe, arb, arr, bid, zib, zib, garr, hal, hal, jat, jat, lan, lan, d-l, sar, dom), guziac (aiz, aiz, ame, ame, azka, azka, aih, bas, lek, bes, bes, ezt, hal, haz, haz, izp, jat, lak, lak, lak, larz, luk, dono, bai, bai, dom), guziak (ahe, ahe, d-s, d-s, zib, garr, jats, d-l, d-l, sar, zur, zur, zur, mil), guzian (bas), guzietaic (bas), guzietaric (ame, lek, d-l), guzietarik (aiz, zib, sar), Guzietarik (d-s), guziez (ame), guzik (urk, urk), guzitzat (haz)

haboro (2): haboro (bar, osp)

haiek (718): aiyeri (ur-p), éhiénn (hen), éhienn (hen), équienn (hen), ayhuen (ur-p), eien (bir), eier (bir), ekiénn (hen), ekien (bir), equien (bir), haien (etx, etx, gar, ida, ida, l-z, l-z, l-z, irur), Haien (alo, l-z, irur), hên (bai, bai, bai, bai), héin (a-z, a-z), héén (beh, ito, ito, ito, ost, ost), Héén (ost), haïndaco (a-z, g-zo, l-s, mend), haïndako (aiz, beh, garr), Héènn (bit), héèr (bit), héér (ost), Héèr (bit), Héér (ost), héc (ost), héen (aint, beh, bun, bun, bun, iba, dona, dona, dona), Héen (aint), Héién (iba), haien (ürr, bar, bid, etx, gar, iri, laka, l-a, mit, urd, urd, urd, bil), Haien (ürr, etx, laka, urd), héien (lah, lah), héier (ilh), Héier (ilh), héierri (lah), Héierri (lah), haindaco (a-m, a-zo, larz), haindako (jats), hékién (don), hékien (ain, ain, ain, ain), hén (hau, hau, hau, hau, hau, iba, l-s, l-s), Hén (l-s), harien (ürr, esk, esk, esk, mit), Harien (esk), hœtan (o-z), hétane (ost), hauyen (esp), hayën (g-z, g-z), Hayën (g-z), hayein ( $z-p)$, hayek (bil), hayen (ainh, ainh, amo, amo, amo, arü, arü, altz, beho, sarr, sarr, ünd, ünd, etxa, etxa, iru, larr, larr, mau, pag, pag, pag, pag, ure, bil, bil), Hayen (ainh, arü, beho, sarr, ünd, larr, mau, mau, mit, bil), héyen (a-z, kan, kan, kan), Héyen (kan, lah), hayer (amo, g-z, pag, bil), Hayer (amo, iru, pag), Héyer (a-z), hayetaco (arro), heïen (buz, buz, buz, meh, meh), Heïen (a-m, buz, meh), heèn (azka, azka, azka), heïn (garr), heén (azka), hec (aha, ame, ame, arb, arr, arh, arh, arü, azka, altz, aih, beho, b-l, ban, bas, bid, bir, lek, lek, lek, lek, bun, g-z, etxa, gab, get, hel, haz, haz, hen, hoz, iru, iru, izp, jats, jut, lak, lekb, lah, lah, lan, lan, luh, luk, mak, mar, mar, mend, müs, ora, o-z, ort, arro, d-g, d-i, sen, bai, dona, suh, u-g, u-h, u-h, ure, uzt), Hec (ban), heeen (leko), heek (men), heen (aha, aint, arn, aih, aih, be-l, d-s, d-s, d-b, d-b, gab, gab, gam, gam, gam, hel, izp, jut, jut, jut, lan, larz, larz, larz, leko, men, men, men, mend, mend, mend, d-g), Heen (aha, arn, d-s, gam, hel, hel, izp, leko, men), He-en (aih), heer (aha, beh, d-s, gab, gab, gab, izp, izp, izp, jut, jut, jut), Heer (aha, hel), heguien (hir), heia (izt), heiec (b-l), heiek (bard), heien (a-m, bard, bard, bard, bard, bir, lek, lek, lek, hoz, hoz, hoz, ilh, ilh, iri, izp, izt, izt, izt, l-b, l-b, mug, ort, ort, ort, ort, d-i, u-g, ure, uzt), Heien (bida, bida, izt, l-b), heier (bard, bard, hoz), heikien (zib), hein (d-b, garr, l-b, urk, ur-p, uzt, uzt), heiyen (mug), hek (ahe, beh, beh, bida, buz, sarr, zib, d-b, ünd, garr, garr, garr, haz, haz, ilh, l-b, l-o, l-o, luh, d-l, d-g, sar, zur), Hek (ahe), hekieï (sen), Hekieï (sen), hekiei (b-l, b-l), hekiek (d-l), hekien (ahe, ahe, arb, arr, b-l, b-l, zib, sen, sen, sen), Hekien (ahe, arb, arr, azk, azk, lar, d-l), hen (a-m, zar, zar, alo, alo, anh, anh, anh, arh, arh, arm, arm, arü, ürr, altz, altz, altz, ban, ban, ban, bar, bar, be-l, be-l, be-l, be-l, bid, bid, bid, soh, soh, soh, soh, soh, sarr, ünd, ezt, ezt, etxa, gar, g-i, g-i, g-i, jats, jats, jats, lak, laka, laka, lekb, lekb, lekb, lig, lig, larr, osp, osp, osp, l-a, l-a, l-a, l-a, l-a, l-o, l-o, mar, mar, men, mit, müs, müs, müs, o-z, o-z, o-z, izu, izu, izu, arro, arro, s-g, s-g, s-g, zal, zal, zal, zal, dom, dom, suh, ath, ath, irur, u-h, u-h, ure, ure, z-p, z-p), Hen (zar, alo, anh, arh, arm, altz, ban, bar, g-i, lekb, lig, osp, l-o, mar, müs, o-z, arro, s-g, dom, u-h), 
hequien (get, d-i, hir, hir, urr, urr), her (a-m, aint, zar, anh, arh, arm, altz, ban, be-l, bun, soh, gar, jats, lekb, lig, l-s, las, izu, arro, arro, d-g, irur, u-h, z-p), Her (ürr, bid, gar, hau, mit, o-z, s-g), heren (aiz, lek, d-g, d-g, u-g, u-g), Heren (aiz, aiz), hetaco (eih, luh), hetan (ahe, be-l, d-s, soh, garr, hel, hoz, jats, larz, ure), hetaric (hal, jat), hetarik (luh), heyec (ame, eih, ur-p), heyek (haz), heyen (ainh, ame, ame, ame, arb, arb, a-zo, a-zo, arr, a-s, a-s, a-s, bas, eih, lek, bes, bes, bes, g-zo, g-zo, esp, ezt, gab, gab, hal, hal, hal, haz, haz, iho, iho, iru, its, its, its, jat, jat, jat, lak, lak, lak, lan, lan, lar, lar, las, las, ald, luk, luk, luk, mak, mak, mak, mak, meh, mug, ora, ora, d-i, d-i, sar, sar, zur, zur, zur, dono, dono, dono, dona, suh, urk, urr, urr, mil, mil, mil), Heyen (ame, a-zo, bas, g-zo, ezt, iho, iru, las, ald, luk, suh, urk), heyer (ame, ame, a-zo, a-s, bas, beho, eih, lek, lek, g-zo, haz, haz, jats, luk, ora, zur, suh), Heyer (a-s, lak, ora), heyerri (bes, mug), houac (alt, amo, g-z, hau, mau, pag), hourak (alo)

hain (112): haï (a-m, aiz, a-z, arh, arm, altz, bit, bes, g-z, gab, gam, garr, hel, hau, iba, iba, jats, jats, l-b, luk, mit, mug, ost, dona, u-h), Haïn (aha, aiz, a-z, arm, arü, azka, bas, g-z, sarr, ünd, etxa, gab, gam, gar, iba, ida, ito, jats, mend, mit, ora, ost, o-z, pag, ath, ure), haïne (ost), haïnian (bai), haïnn (bit), Haïnn (hau), Hagn (etx, laka, l-z, bil), Haiñ (mau), hain (amo, arn, ürr, azk, azka, azka, bir, lek, esp, esk, gab, ilh, ilh, ilh, ilh, iri, jut, jut, jut, l-b, l-b, l-b, l-b, larz, meh, leko, mend, ort, izu, d-i, zur, irur, irur, ur-p, uzt), Hain (amo, a-zo, ürr, be-l, soh, ezt, g-i, ilh, l-b, men), haine (ilh), Hainsari (lak), hoïn (aha, eih, zar), hoin (aint)

hain sarri (2): Haïnsarri (eih)

hainbertzenarekin (7): Haïnbértcénarékin (ain), Haïnbértsénaïquin (1-s), Haïnbertcenarekin (arb), Hainbercenarekin (iri), Hainbertcerenarekin (b-l), Hainbertzénarékin (lah), haïn bertcenarekin (arr)

hainbeste (1): haïmbestetaano (gam)

hainbestenarekin (13): Haïmbestenaéqui (g-z), Haïmbestenarequi (s-g), Haïnbestenaeki (alo), Haimbestenaïkin (d-b), Haïnbestenarekin (a-m), Haimbestereki (esk, arro), Hainbestereki (bar), Hainbeztenarekin (a-s), Hambesténarekin (ainh), Hambestenaïquilan (a-zo), Hambestenaekila (g-zo), Hanbesténarékin (kan)

haizatu (3): haizatiac (arn), haïzçatouac (ain), hachatouac (sen)

hala (21): hala (alt, alo, alo, d-b, garr, ilh, ilh, l-b, laka, larr, l-a, izu), Hala (alt, d-b, l-b, laka, larr, l-a, izu), Halako (d-l), halhan (beh)

halaber (4): Alaber (zib), halaber (meh), Halaber (d-l), halaere (a-zo)

hala-hala (1): Halahala (osp)

halarik ere (1): Haléican éé (alt)

halere (1): halere (g-zo)

han (70): an (bir), han (ain, alo, a-m, ame, amo, a-z, arb, arr, arm, eih, ürr, a-s, beh, ban, bar, bas, d-s, g-z, zib, ezt, etx, etxa, gar, g-i, hau, ilh, iru, jut, l-b, lig, lah, l-s, l-z, l-a, luk, mar, mend, mit, müs, ora, pag, arro, s-g, sar, sar, sar, bai, irur, bil), Han (bard), handic (b-l, b-l, buz, dono, urr), handik (zib, leko, d-l), hanti (zar, altz, l-a), hantic (aint, alt, izp, laka)

handi (69): handi (anh, a-z, arh, eih, ürr, altz, aih, bar, bas, bit, bes, d-s, buz, sarr, zib, ünd, garr, hel, haz, hau, iri, jut, lekb, lan, larz, larr, ald, l-o, luh, luk, men, mend, mit, ora, urd, o-z, zal, dono, dona, dom, suh, u-h, urk, uzt, bil), handia (lek, haz, izp, ald, ald, luh), handiagouk (altz), handibat (aiz, alt, alo, arü, kan, esk, müs, hir), handibatetan 
(mug), handienekoa (zib), handit (larr), handitan (lah), handy (alt), haoundi (iba), haudibat (lar), haundia (b-l), haundibat (zur)

handik eta (17): handic eta (b-l, get, hoz)

hango (3): Hanco (alt), hango (aiz), Hango (d-1)

hara (1): Ara (hau)

haran (11): haran (arb, arr, azk, bes, hal, its, jat, hir, sar, urr, uzt)

har-botxu (3): har botchu (lig, irur), harbotsi (d-g)

harpeña (1): harphegnabaten (pag)

harrapatu (1): arrapatzen (aiz)

harri (112): arria (bir, ur-p), hari (luh), haria (hau, lig), hariac (etx, lak, l-z), hariën (g-z), harien (alo, l-o, müs, d-i), harri (azka, ban, bun, iri, iru, urk), harria (beh, hel, jat, jats, jut, luk, leko, dono, bai, mil), harriac (a-z, arb, arr, arm, ban, bard, bit, lek, bes, get, hal, hen, hoz, iru, lah, lar, l-a, mend, hir, dona, suh, irur, urr), harriak (aiz, ahe, bida, d-s, buz, zib, esp, gam, garr, ilh, l-b, d-l), harrién (ain, ost), harriaren (a-s, larz, las, urd), harrien (aha, a-m, amo, arh, arn, eih b-l, be-l, bid, soh, d-b, esk, etxa, gab, gar, g-i, its, laka, lekb, osp, mau, meh, men, ora, ort, izu, pag, arro, s-g, sen, zal, ath, u-h, ure, uzt, z-p), harrik (altz)

harrigarri (1): harrigarrico (ezt)

harritu (2): haritcecoua (mar), harritséco (ito)

harrobi (1): harrobia (izu)

hartako (7): hartaco (altz, haz, iho, izt, irur, uzt), hartako (bar)

hartan (127): lekhuhartan (ilh), artan (bir, ur-p), hartan (a-m, aha, aint, arn, beho, eih, aiz, ain, zar, ahe, ahe, ainh, alt, alo, amo, anh, a-z, arb, a-zo, arr, arm, arü, ürr, a-s, azk, altz, aih, beh, b-l, ban, bard, bas, be-l, bid, bida, lek, bes, bun, buz, kan, g-z, g-zo, soh, sarr, zib, zib, d-b, ünd, esp, esk, ezt, etx, etxa, gam, gar, garr, g-i, hel, hal, haz, hoz, iba, ida, iho, iri, iru, izp, izt, ito, jat, jats, jut, l-b, lak, laka, lekb, lah, lan, larr, lar, las, osp, l-z, l-a, l-o, luh, luk, mak, mau, meh, leko, mit, mug, müs, ora, urd, o-z, ort, izu, pag, arro, s-g, d-l, d-g, d-i, sen, hir, sar, zal, zur, bai, dona, dom, suh, ath, u-g, u-h, urk, ure, uzt, mil, bil, z-p), hartane (ost), hartann (bit, hau, hen), hertan (men), hartara (anh)

hartaz (7): hartaz (haz, haz, luk, d-g, sen, u-g)

hartu (46): hartcen (eih), har (izp), hartu (zal), hartucik (altz), harturic (arro, z-p)

haserre (1): hassarre (b-l)

haserretu (3): haserretsuac (hal, jat), hassarretu (its)

hasi (3): hasi (ahe), hastian (lekb, urr)

hatsgorapen (1): hatsgorapenac (mug)

hau (42): haü (zib), haütaco (mug), hau (zib, luh), hauc (luh), hauetan (luh), hauk (esp), haukiek (zib), haukien (zib, zib, zib, iri), Haukien (iri), hauyek (luh), hauyen (luh), hoc (ost), houk (altz, altz, altz), hounec (arh, arm, ürr, soh, etx, etxa, hel, hen, jut, lah, larr, l-z, l-a, l-o, mau, ora, pag, arro), hounek (alo, altz, bar, mit), Hounek (garr)

haur (159): haürac (g-z), haürak (gar), haürec (aiz, ure), haürrac (ahe, ainh, arm, arü, ürr, haz, iru, jats, luk, mau, mit, ort, s-g, bai, dom), haürrak (sarr, ünd), haürrari (ahe), haürrec (bun, gab, d-g), haöurrèc (hen), haour (garr), haourac (ain, kan, etx, iba, l-s, l-z, l-a, o-z, irur), haourak (alo, bar, men), haourrac (aha, aint, zar, a-z, a-zo, arh, altz, bard, bit, g-zo, hel, ito, jut, lah, ost, sen, dona), haourrak (altz, beh, gam, garr, don, ath), haourrek (altz, ilh, l-b), haurac (hau, ida, lak, lig, larr, luh, müs, sar, urr, z-p), haurak 
(zib, l-o, bil), haurec (anh, azk, urd, u-g), haurrac (aih, alt, a-m, ame, arb, arn, arr, beho, eih, b-l, ban, bas, be-l, bid, bir, lek, bes, soh, esk, ezt, etxa, g-i, get, hal, hoz, iho, iri, izt, its, jat, laka, lekb, lan, larz, lar, las, mak, mar, meh, mend, mug, ora, izu, pag, arro, d-i, hir, zal, dono, suh, u-h, ur-p, uzt), haurrak (a-s, bida, d-s, buz, d-b, izp, d-l, zur, urk, mil), haurrec (amo, azka, ald, osp), haurrei (bas, bid), haurrek (esp)

hauteman (1): hauteman (anh)

hautsi (7): haüsteco (lekb), haoustéco (bun), hausteco (mend, urd, pag), hausten (lek), hausteranocoan (lak)

hegadora (1): hegadorac (ame)

hegi (7): hégian (l-b), héguian (lah, mug), hegian (zib), heguian (arb, arr, ilh)

hein (7): hégnïn (g-z), heïnian (arm, hel), hegnan (gab), hegniala (alo), heguinean (mil), heineraino (urr)

heldu (127): héldou (ain, kan, ost), héldu (l-s), heldi (mak, meh), heldiac (a-z), heldou (aha, eih, zar, a-s, bit, lek, bun, buz, gam, hel, hen, iba, iba, izt, jut, ald, ald, luh, ora, sen, don, dona), heldu (ahe, aih, aint, aiz, a-m, ame, amo, anh, arb, arb, arm, arn, arr, arr, arh, azk, azka, a-zo, beho, b-l, bard, bas, bid, bida, bir, bes, d-s, g-zo, zib, d-b, d-b, esp, ezt, gab, garr, garr, get, hal, haz, hoz, iho, iri, iru, izp, izp, its, jat, jats, lak, lah, lan, larz, lar, las, osp, luk, mau, leko, men, mend, mug, izu, d-l, d-g, d-i, hir, hir, sar, dono, bai, dom, suh, u-g, u-h, urk, ure, ur-p, urr, uzt, mil), helduac (iri), helduciénac (ito), heldu_cirelaric (mar), helduouc (mend), helduzienak (zur), heltcén (don), heltziari (l-a), heltsen (l-s, ald)

heltier (1): heltier (ath)

heltu (13): heltu (g-z, etx, etxa, lig, osp, l-z, mit, urd, o-z, o-z, zal, irur, bil)

heltura (5): heltua (urd, ath), heltura (urd, arro, arro)

hemen (3): En (dom), hemen (zib, garr)

herri (855): érrirat (beh), beri (luh), erri (beh), erriain (beh), erriaen (beh), erriaren (ur-p), errirat (ur-p), erritan (beh, bir, ur-p), héri (kan, kan, kan, etx, etx, hau, hau, l-s, l-s, l-s, l-s, o-z), hériain (hau, l-s, l-s), hériaen (etx, l-z, o-z), hérialat (etx), hériën (gz), hériarén (ain), hériaren (kan, etxa), Hérico (kan, g-z, etx, hau, l-s), hérilat (o-z), hérirat (kan, d-g), hérri (ain, iba, ost, don), hérriaine (ost, ost), hérrian (ito), hérriarén (ain), hérriaren (don), hérriétan (ain), Hérrico (ain, bit, hen, iba, ito, lah, ur-p), hérrira (bun), hérrirat (ain, ito), hérritánn (hen), heri (alo, alo, azk, d-s, g-z, g-z, g-z, etx, gar, ida, ida, l-z, l-z, l-z, l-a, l-a, l-o, l-o, luh, luh, mar, mau, mau, mau, müs, müs, zal, dom, bil), heriaën (hau), heriaen (alo, arü, men, müs, d-i), heriain (bid), heriala (alo, hau, lig, l-a, irur), herialat (gar, ida, l-z), herian (l-o), heriaren (azk, azk, azk, gar, gar, ida, ida, ida, lig, lig, l-a, mar, mar, mau, leko, d-l, d-i, dom, bil), heribatetan (gar, bil), heribatian (l-o, mar), herico (ida, mar), Herico (azk, l-z, müs), herien (ida, müs), Heriko (gar, l-o, mau, bil), herila (l-o, mau, müs, pag, bil), herilat (mar, men), herirat (azk, d-i), heritan (alo, azk, lig), herrîtan (bai), herretic (hir), herri (aiz, a-m, aha, aint, arn, beho, eih, zar, zar, ahe, ahe, ahe, ainh, ainh, ame, ame, ame, ame, amo, amo, anh, anh, a-z, a-z, arb, arb, a-zo, a-zo, a-zo, arr, arr, arh, arh, arm, arü, ürr, ürr, a-s, a-s, azka, azka, altz, altz, altz, aih, aih, b-l, ban, ban, bar, bar, bar, bard, bas, bas, be-l, be-l, be-l, bit, bit, bid, bid, bida, bida, bir, lek, bun, bun, bun, buz, g-zo, g-zo, g-zo, soh, soh, soh, sarr, sarr, d-b, d-b, ünd, ünd, esp, esp, esp, esk, esk, ezt, etxa, etxa, gab, gab, gam, gam, garr, garr, garr, g-i, g-i, get, get, hel, hel, hal, hal, haz, haz, hen, hoz, iba, iho, iho, 
iho, ilh, iri, iru, iru, izp, izt, izt, ito, ito, its, jat, jat, jats, jats, jut, l-b, laka, laka, lekb, lekb, lekb, lig, lah, lah, lan, lan, larz, larz, larr, larr, larr, lar, las, las, las, ald, ald, osp, osp, luk, luk, luk, mak, mak, leko, leko, men, men, mend, mend, mit, mit, mug, ora, ora, ost, o-z, ort, izu, izu, izu, arro, arro, s-g, s-g, d-l, d-g, d-i, sen, don, don, hir, hir, sar, zal, zal, zur, dono, dono, bai, dona, dom, suh, ath, ath, u-g, u-g, u-h, u-h, ure, ure, ur-p, urr, uzt, uzt, mil, mil, bil, z-p, z-p, z-p), Herri (ahe, altz, bar, haz, izt, uzt), herria (aha), herriaan (bir, bes, bes, hel, hel), herria-an (aih, aih), herriain (aint, a-m, bit, gab, gab, gam, garr, iba, jats, bai), herriaat (hel), herriaen (zar, ainh, altz, altz, bir, bun, buz, g-zo, sarr, sarr, lan, osp, osp, leko, pag, d-g), herriain (a-z, azka, azka, beho, bid, bid, iba, larz, mend, izu), herriala (g-i, larr, osp, s-g, zal, ath, z-p), herrialat (be-l, soh), herrian (arh, arh, bard, bard, bas, bas, iba, ilh, ilh, ito, l-b, l-b, ald, ath, urk, urk), Herrian (lar), herriarènn (bit), herriarénn (hen), herriaren (aiz, a-m, arn, eih, zar, ahe, ahe, ainh, ame, amo, amo, anh, a-z, arb, arb, a-zo, arr, arr, arm, arü, arü, ürr, ürr, a-s, a-s, b-l, b-l, ban, ban, bida, lek, lek, d-s, d-s, buz, soh, d-b, d-b, ünd, ünd, esp, esp, ezt, ezt, etxa, g-i, g-i, get, get, hal, haz, hen, hoz, hoz, iho, iho, iho, iri, iri, iru, iru, izp, izp, izt, its, its, jat, jat, jut, jut, laka, laka, lekb, lah, lah, lan, larr, lar, lar, las, ald, ald, luk, mak, meh, leko, mend, mit, mit, mug, mug, urd, urd, ort, ort, pag, arro, s-g, s-g, d-l, d-g, sen, sen, hir, sar, sar, zal, zur, dono, dono, bai, dona, dona, dom, dom, suh, suh, u-g, u-g, u-h, u-h, ure, ure, ur-p, urr, uzt, uzt, mil, mil, z-p), herriat (zar, bir), herri'tan (ban), herribatçouetan (arm), herribatean (lar), herribatetan (arü, bes, urd), herribatian (pag), herrico (bes), Herrico (a-m, aha, arn, beho, eih, zar, ainh, ame, amo, anh, a-z, a-zo, arh, arm, arü, azka, aih, b-l, ban, bard, bas, be-l, bid, bir, bes, bun, d-s, g-zo, soh, sarr, ünd, esk, ezt, etxa, gab, g-i, get, hel, hal, iri, iru, izp, its, jat, jut, lekb, lig, lan, larz, larr, las, ald, osp, l-a, luk, mak, leko, mend, mit, mug, ora, urd, ost, o-z, ort, izu, pag, arro, s-g, d-g, d-i, sen, hir, zal, dono, bai, dona, dom, suh, u-g, u-h, ure, urr, z-p), herrien (bida, gam, ath), herrietan (b-l, bard, bas, bes, d-s, garr, haz, ilh, iri, l-b, mug, ort, sen), herriko (ahe, aint, jats, men, sar, zur), Herriko (aiz, arb, arr, ürr, a-s, beh, bida, lek, buz, d-b, esp, gam, garr, hoz, ilh, jats, l-b, laka, men, don, sar, zur, ath, urk, mil), herrila (ainh, ürr, etxa, laka, mit, urd), herrilat (arü, altz, bar, sarr, ünd), herrira (a-m, a-s, b-l, esp, hal, iru, jat, jut, lekb, lar, ort, izu, don, sar, bai, ure), herrirat (aha, aint, arn, beho, eih, aiz, ahe, ame, amo, anh, a-z, arb, a-zo, arr, arm, azka, aih, ban, bit, bid, bida, lek, bes, d-s, buz, g-zo, zib, d-b, ezt, gab, gam, garr, get, haz, hen, hoz, iho, ilh, iri, izp, izt, its, jats, l-b, lah, lan, larz, las, luk, mak, leko, mend, mug, ost, d-l, sen, hir, zur, dono, dona, dom, suh, u-g, urr, uzt, mil), herriren (bar, esk), herritan (arn, beho, bida, lek, buz, ezt, hoz, iho, izp, its, jut, larz, meh, pag, d-g, d-i, zur, dona, suh, u-g, urk, urr), herritto (urd), herrriaren (hal), hessi (mak)

herriño (1): heriignobatian (d-g)

herrixka (16): herichka (zib, meh), Herichka (zib), herichkaren (zib), herisca (lak, lak), Heriscaco (lak), heriscarat (lak), heriscaren (lak, lak), herrichca (iri, izt), herrichka (hal, jat, d-1), herrisca (aint)

hertsatu (5): hersatiac (aih), hersatuic (a-zo, g-zo), hertzatuac (ame), hetxatuac (ure)

hi (62): hi (esk, lig, mau), hii (a-m, ath), hire (a-s, zib, hel), hiri (ame, anh, a-z, arm, arm, ürr, beh, b-l, bard, bit, bid, bes, sarr, ünd, gab, gam, gar, gar, garr, g-i, get, hoz, iba, ilh, iru, jats, l-b, lah, larz, las, ald, luk, mak, mar, meh, men, mit, ost, ort, d-g, d-i, don, dono, bai, bai, dona, u-g, mil, bil, bil, z-p), hitaz (sen), iri (bir, ur-p) 
hiaur (1): hiaouri (jut)

hil (7): hil (ahe, ahe, arn, lar, mug), hila (iho), hilabateko (mil)

hilabete (45): hilaïteco (gab, ost), hilabétéco (hau, lah), hilabetean (ame, get), hilabeteco (aha, amo, altz, b-l, g-i, jut, mak, mau, ora, ort, ure, uzt), hilabeteko (esp, gam), hilabethean (izt), hilabethian (hal, jat), hilabetian (aiz, d-g), hilabetiaren (u-g), hilabetin (sarr, ünd), hilaiteco (larz), hilhaiteko (mug), hillabetian (urr), hillete (bard), ilabeteco (men), ilaiteco (bid), ilateco (bir), ilhaïteco (bai), ilhaitian (jats), ilhabétéänn (hen), ilhabéthienn (beh), ilhabeiteco (sen), ilhabetearen (arn), ilhabetian (suh), ilhabetiaren (d-s), illeteco (ur-p)

hiri (140): kohirian (l-b), hiaen (altz), hiiaen (etx, l-z, o-z), hiiën (g-z), hiiaren (hau, men, pag), hiraren (d-i), hiri (arn), hiria (bun), hiriaên (zur), hiriaan (hel), hiria-an (aih), hiriaïn (a-z, beh, gab, jats, bai), hiriaën (ainh), hiriaïne (ost), hiriaen (alo, bir, osp), hiriain (azka, iba, ilh, larz), hirian (arh, bard, d-g, urk), hiriarén (ain), hiriarènn (bit), hiriärénn (hen), hiriaren (aiz, a-m, aha, aint, arn, beho, eih zar, ahe, ame, amo, anh, arb, a-zo, arr, ürr, a-s, b-l, ban, bas, be-l, lek, buz, kan, g-zo, soh, sarr, zib, d-b, ünd, esp, ezt, etxa, gar, g-i, get, hal, haz, hoz, iri, iru, izp, izt, its, jat, jut, lak, laka, lekb, lig, lah, lan, larr, lar, las, ald, l-a, l-o, luh, luk, mak, mar, mau, meh, mit, mug, ora, urd, o-z, ort, arro, s-g, sen, don, hir, sar, zal, dono, dona, suh, irur, u-g, u-h, ure, ur-p, uzt, mil, bil, z-p), hirien (esk, garr), hirietako (zib), hirila (g-z), hiriren (bar), hirriain (izu), hirriaren (ora)

hirixka (1): hirichka (zib)

hiru (1): hiruetan (lar)

historia (16): iztoria (müs), historia (bid, lek, lig), Historia (arü), istoïa (alt, alt), istoria (arh, arh, ito, ito, urd, urd, u-h, u-h), iztoria (müs)

hitz (2): hitz (garr, garr)

hizkuntza (3): hitzcountcia (u-g), hitzcountzia (u-g), hitzkountcia (d-g)

hobe (1): hobia (arh)

hoberen (143): hobêna (zur, bai), hobééna (azka), hobéena (iba), hobéna (aint, zar, a-z, beh, g-z, hau, l-s, ath, z-p), hobénéna (bit), hobéréna (ain, bun, kan, hen, lah), hobe'ena (ban), hobeena (bas, bid, bes, g-zo, d-b, gab, gam, hel, larz, osp, leko), hobena (aiz, ainh, alt, alo, a-m, amo, a-zo, arm, arü, ürr, altz, bar, bard, be-l, soh, sarr, ünd, esp, esk, etx, etxa, gar, garr, g-i, haz, ida, ilh, l-b, laka, lekb, lig, larr, l-z, l-a, l-o, mau, men, mend, mit, müs, urd, o-z, izu, pag, arro, s-g, d-i, zal, dom, suh, irur, u-h), hobenena (a-s, ost), hoberena (ahe, ame, anh, arb, arn, arr, azk, aih, beho, eih, lek, d-s, buz, ezt, get, hal, hoz, iho, iri, iru, izp, its, jat, jats, jut, lak, lan, lar, las, ald, luh, luk, mak, mar, meh, mug, ora, ort, d-l, d-g, sen, hir, sar, dono, dona, u-g, urk, ure, urr, uzt, mil, bil), hoberenac (izt), hoberenena (b-l), obéna (ito), obeena (bir), oberena (zib)

honek (17): hunéc (ost), hunec (bas, be-l, bes, iho, iru, izp, mak, leko, sen, suh, u-h, ure, urr), hunek (azka, zib, sar)

honen (3): hunen (hal, jat), Hunen (esp)

honetako (2): huntaco (meh), huntako (zib)

honetan (2): hountan (ame), huntan (urr)

hor (15): ho (müs), hoez (lig, irur), hor (ainh, ürr, bar, be-l, soh, esk, g-i, larr, mit, arro, s-g, ath)

hori (12): Horek (urd), hori (arm, gab), horrén (ost, don), Horrén (don), horrec (altz, altz, 
iho), horrek (ahe), horren (mil), hotaco (eih)

horiek (28): hoic (luh), hoien (larz), hoiquien (get, get, hir, hir), horiéc (ost), Horién (don), horiétaco (don), horiec (ban, hir, urr, urr), horiek (esp, garr), horien (azk, esp, ezt, sen, dono), oriec (ur-p), orien (ur-p), hoyec (lah), hoyek (sar), hoyen (arr, esp, lah), hoyerri (urk)

horma (49): horma (ain, ahe, anh, arb, arr, azk, aih, b-l, ban, bid, bida, lek, bes, kan, esp, get, hel, hal, haz, hen, iho, iri, izt, its, jat, lah, lar, luh, mak, meh, leko, mug, ort, d-l, sen, sar, zur, dono, bai, dom, urk, ure, urr, uzt, mil), hormac (aiz), hormak (zib), orma (bir, ur-p)

horma-beltz (2): horma belza(ame), horma beltza (bas)

hormatu (1): hormatcen (arm)

horra (10): hora (g-z, l-o, bil), horra (a-z, altz, zib, larr, men, arro, s-g)

horrela (2): hola (d-1), Hola (d-1)

horretan (11): hortan (azka, gab, get, its, larz, l-s, ald, mend, don, dono), hotan (bar)

hotz (122): hot (aha, urd), hotç (osp), hotça (altz), hotçac (its, hir, suh, ure, urr), hots (amo, arü, eih, lek, soh, esp, hau, ito, lig, luk, mak, ost, ath, irur), hotsa (bun), hotsac (ban, mend, z-p), hotx (dona), hotz (ain, aint, a-m, beho, zar, ainh, alo, a-z, arb, a-zo, arr, arh, arm, azk, aih, beh, bas, be-l, bit, bid, bir, bes, d-s, g-zo, sarr, zib, d-b, ünd, esk, ezt, etx, gab, gar, garr, g-i, get, hal, haz, hen, ilh, iru, izp, izt, jat, jats, jut, l-b, lekb, larz, larr, lar, las, ald, l-z, l-a, l-o, luh, meh, men, mug, müs, ora, ort, izu, pag, s-g, d-g, sen, don, hir, sar, zal, zur, dono, u-g, u-h, urk, ur-p, uzt, mil, bil), hotzac (arn, azka, hoz, iho, iri, bai), hotzak (buz, gam, lak, leko, d-l), otz (l-s)

hoztu (10): hostuac (ame, b-l, d-i), hostuic (alt, ida), hotztuac (hel), hoztiac (lan, dom), hoztuak (ahe), hoztuic (laka)

huiatu (4): houiatouac (ain), houlatuilk (garr), huyatuac (urr), uiatuac (bir)

hullantu (2): huillancétic (ito), hullantziareki (ida)

hun (2): houn (eih), hun (a-m)

huna (8): houna (aint), Houna (a-m, aha, eih), hounac (aha, aint), Huna (beho), hunac (beho) hura (533): aren (azk, urd), han (bard, kan), hark (hir), hurra (a-m), -houa (bun, gam, hau, l-b, l-s, pag), -houra (aint, arü, be-l, eih, soh, jut, mau, men, mit, d-i, sen, dona, bil), houraçouçoun (arm), -hourazen (a-s), -hua (gab, leko), -hura (arb, azk, aih, hen, izp, u-g, u-h), -huranzen (mil), hüa (d-b), Hüa (d-b), Hüna (d-b), Haên (zur), haan (hel, leko), ha-an (aih, aih), Haan (hel), haën (hau), haén (ito), Haën (hau), haïndico (bit), haènn (bit), hac (bun), haen (aha, aint, zar, alt, alt, alt, alo, altz, beh, beh, beh, d-b, etx, lig, lig, l-z, o-z, o-z, izu, pag, pag, ath, ath, irur), Haen (alo, altz, bid, etx, l-z, men, urd, o-z, ath), haentzat (zur), hak (garr, garr), hantçat (bard), haréc (l-s), harén (ain, bun), Harén (ain, ito), harèndaco (bun), harénn (hen), haréntçat (ain), haratago (zib), harc (get), harec (izp, lig, s-g), harek (luh), haren (ainh, alo, ame, amo, amo, amo, amo, anh, arb, arn, arr, arh, arm, arü, a-s, azk, azka, ban, ban, bar, bas, beho, be-l, bid, bida, eih, lek, d-s, buz, buz, kan, soh, sarr, zib, zib, d-b, ünd, esp, esk, ezt, ezt, etxa, g-i, g-i, get, haz, hoz, hoz, ida, ida, iho, iri, iru, iru, izp, izp, izt, izt, izt, its, its, lak, laka, lekb, lekb, lah, lah, lan, larr, lar, las, ald, ald, osp, l-a, luh, luh, luh, luh, luk, luk, mak, mak, mar, mau, mau, meh, meh, men, men, mit, mug, ora, urd, ort, ort, s-g, d-i, sen, don, hir, zal, zal, zur, dono, dono, bai, bai, dona, dona, dom, suh, u-g, u-h, urk, urk, ure, ure, urr, uzt, uzt, mil, z-p), Haren (ahe, ainh, a-m, ame, amo, anh, arh, beho, ürr, ban, 
bar, be-l, kan, g-zo, soh, sarr, zib, ünd, esk, ezt, etxa, g-i, ida, iho, iru, izt, jut, laka, lekb, lah, larz, larr, las, ald, osp, l-a, l-o, mak, mar, mau, meh, mit, mug, müs, ora, urd, ort, izu, pag, arro, s-g, d-g, d-i, sen, sar, zal, suh, u-g, u-h, urk, urr, bil, z-p), harendaco (anh, bid, eih, iru, lak, lan, las, ora, d-i), harenn (hen), harentçat (b-l), harentaco (arü, sarr, ünd, lig, l-a, müs, s-g, z-p), harentako (l-o), haren_tako (bil), harentzat (a-s, get, hal, jat), hari (gab), harren (ürr, bes, gar, lekb, arro), Harren (arü, b-l, gar), harrentako (gar), houa (aha, alt, amo, altz, altz, bard, g-z, etx, garr, garr, hel, hel, hau, iba, lig, osp, l-z, men, ost, ost, ath, irur), -houa (a-zo, ilh, o-z), houra (ain, zar, ainh, alo, a-z, a-zo, ürr, a-s, bar, buz, kan, g-zo, sarr, esk, etxa, gar, hen, ida, laka, lah, ald, l-a, luh, luh, mar, müs, ora, urd, urd, arro, s-g, d-g, sen, don, zal, dona, z-p), -houra (g-i, l-o), (houra (ünd, larr), Houra (zar, zar, bit, buz, dona), hourac (arh, be-l, soh, esk, arro, s-g), houracen (ort), hourazen (aiz), hua (aih, beh, bes, izu, dono, urk), -hua (dono), Hua (bes, gab, leko), -huazen (larz), huna (ban, d-g), Huna (anh, azka, d-s, ezt, hoz, izp, jats, lan, larz, las, luk, mend, d-g, bai, u-g, u-h), hunac (anh, a-z, azka, ban, ezt, gab, hoz, izp, lan, las, bai, u-g), hunak (d-s, jats), hura (ahe, ahe, ame, anh, arn, arr, aih, b-l, ban, bas, beho, bid, lek, lek, d-s, d-s, zib, hal, haz, hoz, hoz, iho, iri, iri, iru, izt, its, jat, jats, lak, lan, lar, las, mak, mak, meh, mend, d-l, d-l, hir, sar, bai, dom, suh, ure, uzt), -hura (esp, luk, zur), (hura (bida, urr), Hura (arn, arb, arr, azk, bida, ezt, hal, its, jat, lak, lar, meh, d-l, bai, ure, uzt, mil), huracen (get), hurazen (azka), ua (bir) hurbildu (1): hourbiltsen (luh)

hurtu (1): hurtu (d-g)

ibai (1): ibai (lar)

ibar (17): ibar (alt, amo, be-l, soh, esk, g-i, ida, laka, lig, l-a, urd, s-g, irur), Ibar (lekb, urk), ibarre (gab)

ibili (4): Ibili (iho), ibiliak (d-s), ibilli (bida), ibilten (bun)

ibilzale (2): ibilçale (amo)

ideki (65): idékitcea (aint), idékitéat (beh), idékitcéa (zar), idékitcérat (ain), idékitséra (kan), idékitzéra (lah), idéquitéa (bit, l-s), idéquitcérat (hen), ide’itze’a (ban), ideitcera (gab), ideitcea (leko), ideite (urk), idekhitcera (hoz), ideki (zib, lar, ost), idekitcea (arm, esp, etx, l-z), idekitceat (bida), idekitcera (azk, lar, las, mug), idekitcerat (arb, arn, arr, lek, its, meh, sen, uzt), idekitea (bes, d-b), idekitzea (hel), idekitzeat (azka, bas), idekitzeât (zur), idekitzera (ezt, hal, jat, mak, sar, dom), idekitzerat (ahe, beho, d-s, zib, haz, iri, dono, mil), idequi (hir), idequic (a-zo), idequitcea (bir), idequitcerat (get, hir, urr), idequitzera (iru, bai), idiéïten (l-s), idiitzera (bid), idikitzerat (d-l)

idoki (18): idoïteat (bard), idokiak (ilh), idokitcea (jut), idokitceat (a-m, aha), idokitcera (ort), idokitcerat (d-g, u-g), idokiterat (aih, izt), idokitsen (luh), idokitzea (gam), idokitzeat (iho), idokitzera (jats, dona), idoquiäc (hen), idoquitcera (mar, eih)

iduri (1): uduï (altz)

igan (18): igaan (g-zo, d-b), igaïtentzen (aiz), igan (alo, a-z, a-zo, altz, g-z, ilh, l-b, lig, l-a, men, müs, ath, irur), igân (bai), igann (hau)

igorri (26): Egoriac (ur-p), egorriac (hal, hir), Egorriac (hen), egorriak (sar), igoriac (o-z), igoric (hau), Igoriic (etx), igoriric (l-a, bil), igorriac (jat, uzt), Igorriac (urr), (igorriak) (bida), Igorriak (d-b), igorric (alt, ito), Igorriic (l-z), Igorriik (ath), igorriric (g-i, laka, lar, osp, mar, arro), igorririk (bar)

igual (8): higoal (ahe), higual (get, its), Higual (mak), igoal (anh), igoual (a-s), igual (bir), 
Igual (azka)

ihes (88): ies (bid), iguesi (ur-p), ihès (hau), ihés (ost), ihési (a-z, kan), ihéssi (ain, l-s), ihech (sen), ihejs (ban), ihes (aint, arn, beho, zar, ainh, alo, anh, aih, bar, bas, bir, lek, bes, d-s, buz, g-zo, esp, gab, gam, hel, hal, hoz, ilh, iru, izt, jat, jats, jut, lak, las, ald, luk, meh, mend, pag, dono, bai, dona, ure, uzt), ihesari (suh), ihesi (ahe, arb, arr, azk, azka, bida, zib, get, iri, izp, larz, l-a, leko, mug, d-l, hir, sar, urr), ihessari (bard), ihessi (beh, lah, mak, mau, ora, urd, arro, s-g, zal), ihets (ort), ihex (a-m, dom), ihez (ame, a-zo, a-s, eih, its, men)

ihes egin (1): ihesiten (l-b)

ihur (39): ihour quec (alt), ihourc (ürr, be-1, soh, sarr, esk, g-i, hau, ida, lig, larr, larr, osp, l-z,

l-a, müs, arro, arro, s-g, zal, irur, z-p), Ihourc (etx), ihourcere (müs), ihourk (arü, bar, gar, laka, mit, bil), ihourkere (ainh, alo, ünd, pag, ath), Ihourkere (alo), ihourqué (o-z), ihourquéréc (etxa), ihourquec (g-z)

ihun (1): ihouné (altz)

ikara (2): ikharan (iru), ikharra (zib)

ikaratu (146): hicaratu (ur-p), icaratu (aih, bid, bir), iccaatu (ito), ikaatu (a-zo), ikaratcénn (hen), ikaratcen (esp, get), ikaratcenda (lar), ikaratsen (luh), ikaratu (bida, lek, haz, jats, mend, dono), ikaratuzen (zur), ikaratzen (luk), ikarratu (anh), ikhaatü (d-b), ikhaatou (ost), ikhaatu (alo, altz, ban, bes, g-z, g-zo, etx, ilh, l-b, lig, osp, l-z, o-z, izu, ath, irur, urk), ikharatcén (don), ikharatcen (iri, lan, mar, urd, hir, uzt), ikharatou (ain, eih, zar, arh, bit, buz, hel, iba, izt, jut, lah, ald, ora, sen, dona), ikharatoussén (bun), ikharatu (ahe, ainh, a-m, ame, amo, arn, a-z, arb, arr, arm, azk, beh, beho, b-l, bar, bas, be-l, d-s, soh, sarr, ünd, ürr, esk, ezt, gab, garr, g-i, hal, hoz, ida, iho, iru, izp, its, jat, lak, laka, larz, larr, l-s, las, mak, mau, meh, men, mit, müs, ort, pag, arro, s-g, d-g, sar, zal, bai, dom, suh, u-g, u-h, ure, mil, z-p), ikharatucen (aint, leko, mug), ikharatuzen (aiz), ikharatzen (a-s, azka, gam, l-o), ikharratou (lekb), ikharratoucen (aha), ikharratu (arü, etxa, gar, l-a, d-i, urr, bil), ikhatu (bard, hau), ikhratou (kan), iqharatu (alt)

ikasi (2): ikasi (bida), ikhassi (ida)

ikusi (371): ickouchi (ito), icousi (bid, bid), icoussi (l-s, l-s, o-z, o-z), icousteco (bid, o-z), icusi (amo, amo, aih, aih, bir, jats, hir, hir), icusteco (amo), icustiac (anh), icustiaz (bir), ikhüsteko (d-b), ikhouchi (sen), ikhouchteco (aha, sen), ikhouci (arh, hen), ikhouji (ost), ikhoujtéco (ost), ikhoujteco (iba), ikhous (bit, bun, eih, etx, lig, l-z, irur), ikhousi (aha, ain, alt, alo, alo, arm, ürr, a-s, altz, be-l, buz, buz, kan, kan, g-z, soh, sarr, esk, esk, etx, etx, etxa, gar, gar, hel, hau, jut, laka, laka, lekb, lig, ald, l-z, l-z, l-a, l-a, mit, urd, izu, s-g, s-g, don, dona, ath, bil, bil), ikhousji (iba, iba), ikhousjtéa (iba), ikhoussi (ain, aint, eih, zar, zar, a-zo, arü, arü, ürr, bar, bard, bit, bit, bun, g-zo, etxa, gam, g-i, g-i, ida, ilh, ilh, l-b, l-b, lig, lah, larr, larr, osp, l-o, mau, mau, mit, müs, müs, ora, urd, pag, zal, zal, irur, irur, z-p), ikhoussicin (pag), ikhoussiric (lig, irur), ikhoussissien (bun), ikhoustéco (zar, g-z, lah), ikhoustén (don), ikhousténn (hen), ikhousteco (alt, arh, ürr, altz, be-l, g-zo, soh, esk, etxa, jut, laka, larr, l-a, mau, ora, pag, arro, s-g, dona, ath, z-p), ikhousteko (alo, a-m, buz, sarr, ünd, gam, ilh, l-o, mit, d-g, bil), ikhousten (bar, g-zo, g-zo, ida), ikhoustiaën (hau), ikhoustiaen (osp), ikhoustiaren (g-i), ikhouz (a-s), ikhouzi (aiz, a-s, garr, garr), ikhouzizic (garr), ikhouzteko (garr), ikhujsi (ban), ikhujsten (ban), ikhus (arb, arr, izp, lar, d-i, ure, uzt), ikhusi (ahe, ahe, ainh, a-m, arn, a-z, arb, azka, beho, b-l, b-l, bas, bas, bes, d-s, d-s, zib, d-b, d-b, ezt, ezt, ezt, gab, gab, 
hal, hoz, hoz, iho, iri, iri, iru, iru, izp, its, jat, jats, lak, larz, las, las, luk, leko, leko, mug, d-g, d-i, d-i, sar, sar, bai, bai, dom, dom, suh, suh, u-g, urk, ure, uzt, mil, mil), ikhussi (a-z, beh, lan, mak, mak, u-h, ure), ikhustearen (larz), ikhusteco (ainh, azk, b-l, gab, hal, iru, jat, luk, mak, leko, mug, bai, suh, u-g, u-h, uzt), ikhusteko (bas, zib, ezt, gar, l-b, lak, sar, urk), ikhusten (ahe, a-z, arr, azka, d-s, zib, ida, iri, lar, lar, mug), ikhuzi (ame, ame), ikisi (ur-p), ikousi (ünd, lekb, luh, men), ikoussi (osp, mar, mar, arro), ikoustén (don), ikousteco (a-zo, arm, arm, arü, lekb, mar), ikousteko (ald, men), ikousten (a-zo, a-zo, ald, l-o, luh, luh, mar), ikoustiarén (don), ikujsi (ban), ikujsteco (ban), ikusi (arb, arr, azk, azk, beho, bida, bida, lek, lek, esp, get, haz, haz, izt, meh, meh, mend, mug, ort, d-l, d-l, zur, dono, ur-p, urr), ikus (bida, d-l, hir), ikusizuin (zur), ikussi (anh), ikustearen (lek, get), ikusteco (bida, urr), ikusteko (esp, zur), ikusten (lek, haz, d-l), ilhousi (arm)

ilhain (1): ilhagnez (l-o)

iluminatu (2): illuminatia (u-g), iluminatia (d-g)

ilun (27): Ilhoun (ain, aint, jut, lah, mug, dona), Ilhounaren (lekb), Ilhoune (ost), Ilhun (anh, arb, arn, arr, azk, b-l, bida, bes, izt, larz, d-l, mil), Ilhuna (luk, ure), Ilhunariburuz (aiz), Ilhunian (garr), Ilun (meh), Ulhun (mau), Ulhunaren (l-a),

ilunabar (21): Ilhoun gnabarian (kan), Hilounarrian (aha)Hilhun arrian (ur-p), Hiloun habarriänn (hen), Ilhounaarrian (buz), Ilhounarrian (arm), Ilhoun_naarrian (zar), Ilhuna aarrian (ezt), Ilhunaarrian (mend), Ilhun_aarrian (bir), Ilhunabarrian (bai), Ilhun-nabarrean (sar), Ilhun_nabarrian (hal, jat, jats, las, ort, suh), Ilunaarrian (bid), Ulhun gnabarrian (s-g), Ulhungnabartzian (alo)

ilundiri (6): (hel), Ilhoundirian (ora), Ilhundirian (gab, dom), Ilhunirian (dono), Ulhunduriaékin (beh)

iluntze (84): Ilhunciaikin (a-m), Ilhüntzian (d-b), Ilhounnçiann (bit), Ilhounsian (bun), Ilhountcean (sen), Ilhountcian (eih, iba, izu), Ilhountciarékin (don), Ilhountsian (arh), Ilhountzian (a-s, gam, iho), Ilhuncean (leko), Ilhuncian (a-z, ito, mak), Ilhuntcean (its, uzt), Ilhuntcebatez (lar), Ilhuntcian (amo, g-zo, esp, get, lan, d-g, d-i, u-g), Ilhuntsian (bas), Ilhuntze (iru), Ilhuntzebatez (hir), Ilhuntzian (azka, ban, beho, lek, d-s, hoz, izp), Ilhuntziarekin (ame, haz), Ilhuntziari (lak), Ilhuntzin (u-h), Ilunsian (ald), Iluntchian (zur), Iluntciai (men), lhuntzian (urk), Ulhun ceratcin (bil), Ulhüntsian (g-z), Ulhüntzian (gar), Ulhountzin (esk), Ulhun tcébatez (alt), Ulhun -tcin (ünd), Ulhuncé (ath), Ulhuncian (zal), Ulhunsian (l-o), Ulhuntcian (a-zo, be-l, etx, etxa, g-i, hau, laka, larr, l-z, müs, pag, irur), Ulhuntciari (osp, urd), Ulhuntcin (ainh, arü, altz, sarr, arro), Ulhuntsé (l-s), ulhuntsian (l-s), Ulhuntsian (lig, o-z), Ulhuntzian (soh, z-p), Ulhuntzin (ürr, bar, mit)

inguratu (11): iguratua (zib), inguratia (luk), inguratua (ahe, bida, iri), inguraturic (z-p), inguraturik (bar), ingurukatia (garr), ungüaturic (alt), unguratuic (urd), unguraturic (g-i)

inkaru (1): inkarubat (ida)

instant (43): istantian (a-s), ichtant (get), ichtantian (anh, azka, ban, buz, gam, hau, iho, ilh, iru, jats, jut, l-b, lah, las, ora, izu, d-i, bai, mil), ichtantin (bes), ichtatian (luk), ichtàntiann (hen), instantian (ito), instantiane (ost), istant (mar), istantean (b-l), istantian (arh, bir, ezt, lak, lak, mak, mend, d-g, u-g, u-h, ur-p, z-p), istantien (bard), istantin (sarr, ünd) 
intziri (3): intziriac (iri), intziriak (d-s), intzirinac (bai)

ipuin (4): iphunya (hal, hal, jat, jat)

iragan (37): igaran (ainh, arü, ürr, be-l, soh, sarr, ünd, esk, etxa, gar, ida, laka, larr, osp, l-o, mar, mau, mit, pag, arro, s-g, zal, bil, z-p), igarançenn (bit), iragaiten (bida), iragan (arb, arr, zib, g-i, hal, iri, jat, luk, meh, d-i, sar)

irakatsi (1): erakatx (osp)

irakurgarri (3): iracourgaria (men, men, zal)

iratzarri (173): iartçatiaïkin (aint), iatcarric (aint), iatçartçia (aha), iatzarric (aha), i'atsarci'an (ban), ihatzarririk (d-s), Ihatzartzian (d-s), iartçartcean (lar), iarzaziaquin (bir), iatçarcean (leko), iatçarciaïkin (mend, izu), Iatçarciaekin (g-zo), iatçarciai (men), iatçarciakin (ilh, l-b), iatçarcian (d-g), iatçarciareki (ida), iatçarciarekin (don), iatçaririk (bil), iatçarria (bit), Iatçarria (gab), iatçarric (zar), iatçartçiaékin (beh), iatçartciaëki (altz), Iatçartciaïki (hau), iatçartciaïkin (a-m, a-z), iatçartciaïkine (ost), iatçartciaeki (l-z), iatçartciaikin (gab), iatçartciakin (zar), iatçartcian (ath, u-g), Iatçartcian (iba), iatçartciarekin (anh), iatcarciareki (etx), iatsari (ito), iatsartcian (lig), iatsartsia (bun), iatsartsian (irur), iatsartziarequi (o-z), iatzarcearekin (uzt), iatzariik (ünd), iatzarik (azka), iatzarria (iri, meh), iatzarriic (bes, hel), iatzarriric (bid), iatzartzia-akin (aih), iatzartziaïkin (gam, jats), iatzartziaikin (d-b), iatzartziakin (bas), iatzartzian (alo, bid, zur), Iatzartzian (bes), iatzartziarekin (iho, izp, dono), Iatzartziarekin (dom), Iatzartzin (ünd), iatzarziaakin (hel), iatzarziaikin (azka), iazartzian (u-h), idatzartzian (urk), iharçarcéaréquinn (hen), iharçatu (mug), iharsatciakin (bard), iraçar ciarequi (be-l, soh), iraçartciaikin (a-zo), irartzar siaikin (l-o), iratçarciaequin (alt), iratçarciarekin (bl, mar), iratçarciarequin (amo), iratçaria (pag), iratçariric (müs, urd), iratçarria (eih, ora, suh), iratçarriik (izu), iratçarriric (amo, etxa, g-i, osp), iratçarry (urr), iratçarsiann (bit), iratçartcéan (lah), iratçartcean (izt), iratçartceareki (ure), iratçartciaeki (osp), iratçartciaikin (jut), iratçartcian (ainh, eih, g-i, mak, ora, s-g, zal, z-p), iratçartciareki (pag, arro), iratçartciarekin (esp, laka, las, dona, suh), iratçartciarequin (etxa), iratçartcin (arü, bil), iratçartsiarequi (mau), iratcarcearekin (ort), iratcarciaeki (urd), iratcarciareki (müs), iratcarciarequi (larr), Iratsarciarekin (ald), iratsartsen (luh), iratsartsiaiquin (1s), iratsartsiarékin (kan), iratsartsiequilan (g-z), iratxartxiarekin (arr), iratzarciarekin (luk), iratzaria (lak), iratzaric (l-a), iratzariik (sarr), iratzaririk (gar), iratzarria (bas, hal, jat, bai), iratzarriric (esk, ezt), iratzarririk (ürr, mit), iratzarsiareki (ürr), iratzarsiarekin (mit), iratzartciarekin (hoz), iratzartcin (sarr), iratzartzearekin (ahe, hal), iratzartzearequin (ame), iratzartziakin (larz), iratzartzian (arh), iratzartziarekilan (gar), iratzartziarekin (arb, buz, ezt, jat, lak), iratzartziarequi (l-a), iratzartzieki (esk), Iratzartzin (bar), iratzarzean (mil), iratzarzian (bai), iratzarziarekin (a-s), iratzarziaikin (beho), irazarriakin (lan), irratçarciarequin (d-i), irrazarririk (lekb), irrazartzian (lekb), itzartziarequin (iru), iyatzartcian (meh), iyazar ciarekin (ur-p), Yatçartcearekin (arm), Yatzarcearekin (iri), yatzarziarekin (aiz)

iratzartu (5): iatçartua (leko), ihatçartu (bida), jatzartu (garr), iratzartu (arn), iratzarturic (arn) ireki (2): oroidekiak (l-b), irekiseat (ur-p)

irus (92): ious (alt, alo, altz, g-z, etx, hau, l-z, o-z, pag, ath), irüs (d-b), irous (ainh, amo, a-z, arh, arü, bar, be-l, bun, soh, sarr, ünd, esk, g-i, ida, ilh, jut, l-b, laka, osp, l-a, mar, mau, men, mit, müs, urd, izu, arro, s-g, don, z-p), irousj (ost), irouss (bit), iroussac (a-zo, g-zo, l-s), irouz (a-s, etxa, garr, lig, larr, irur), iruj (iba), irus (a-m, ürr, gab, lan, larz, 
luk, mug, u-h), Irusac (bas), irusak (urk), irutzak (mil), iruz (ame, beh, bard), urrus (bid), urrusac (dom), urus (arb, arr, azk, aih, bida, bir, bes, esp, get, mak, meh, ure, ur-p), urusac (iri, dono), urusak (lek, haz, leko, zur), uruz (its, l-o)

isla (4): isla (alt, l-a), islaca (etx, l-z)

istil (2): istil (larr), istilla (larr)

istorio (53): hichtorio (izt), historio (izt), historioa (ame, ame, bid, bun, bun), Historioa (luh), historioua (mend, mend), ichtoïa (hau, l-s, l-s), ichtoria (bar, bar, be-l, be-l, d-s, d-s, soh, soh, ilh, ilh, l-b, l-b, larz, larz, osp, l-a), Ichtoria (osp), Ichtorio (iho), ichtorioa (bit, bit, iru, iru, l-a, ost, suh, suh), ichtorioua (buz, iba, iba, ost), ichtoriouaren (buz), ichtoriua (bes, bes, zur, zur), istorio (luk), istorioa (luh, don), ichtorioa (beho)

itandi (1): ithandi (don)

ito (1): itho (garr)

itsaso (2): itchasso (ida), itzazo (ame)

itzali (17): itçaldou (ost), itçaldu (ort, hir), itçali (eih, las), itsaldu (bard), (itsali (ban), itzaldu

(bes, d-b, hoz), itzali (arn, d-s, ezt, lak, luk, u-g), itzaltzentzou (gam)

itzul (2): itçul (hir), itzul (lar)

itzuli (2): itzuldu (ame), utçul (urd)

ixtant (1): ichtantian (beho)

izan (831): içan (aha, aint, eih), Içan (a-m), içana (aha, eih), izan (a-m, arn, beho), izanic (arn, beho), Izantzen (beho), 'zan (sarr), hiçan (bard), içan (ain, ain, ain, zar, zar, ainh, ainh, alt, alt, amo, amo, amo, anh, anh, a-zo, a-zo, a-zo, a-zo, arm, arü, azk, azk, azk, azk, altz, altz, beh, beh, b-l, b-l, b-l, bard, be-l, bida, bida, g-z, g-z, g-zo, g-zo, g-zo, g-zo, soh, soh, soh, esp, esp, etx, etx, etx, etx, etxa, etxa, gab, gab, g-i, g-i, g-i, g-i, hel, hel, hau, hau, hoz, iba, iba, ida, ida, ida, ilh, iru, iru, iru, iru, iru, izt, izt, izt, izt, izt, izt, its, its, its, l-b, laka, laka, laka, lah, lah, lah, lah, larr, larr, las, las, las, las, las, osp, osp, osp, osp, l-z, l-z, l-z, l-z, l-z, l-a, l-a, l-a, l-a, l-a, mak, mak, mak, mak, mak, mak, mak, mar, mau, mau, meh, leko, leko, leko, leko, men, men, men, men, mug, mug, mug, mug, mug, mug, mug, ora, ora, ora, urd, urd, ort, ort, ort, ort, izu, arro, arro, arro, s-g, s-g, s-g, d-g, d-g, d-g, d-g, d-i, d-i, sen, sen, sen, sen, sen, don, hir, hir, hir, zal, zal, dona, ath, u-g, ure, urr, urr, urr, urr, bil, bil, bil, z-p, z-p, z-p, z-p), Içan (ain, anh, g-z, hel, hau, ida, jut, lan, mar, mau, mend, müs, ora, urd, ort, s-g, d-i, zal, ath), içana (amo, anh, a-z, its, jut, l-a, ort, d-i, dona), içanaatic (a-zo, g-zo, hel, iba), içanaatik (ilh, mend), içanac (ain), içanagatic (soh, hir, bil), içana_gatik (men), içanagatikan (mak), içanartio (mit), içanatic (gab), içanâtic (mug), içanatik (l-b), içanbedi (bit), içancen (ito), Içancen (pag), içanez (l-z), içanic (arm, bit, izt, ito, laka, ald, osp, d-g), içanik (leko, u-g), içanikan (arh), içann (bit, hau, hen, hen, hen, hen, hen), içaten (osp), içatu (its, its), ican (anh, altz, etx, mend, mug), icanez (etx), isan (ban, ban, ban, ban, ban, ban, kan, kan, kan, kan, l-s, l-s, ald, ald, ald, luh, luh, luh, luh), isanarté (ur-p), isanic (ürr, kan, l-s, mit), isantsén (l-s), issan (bun, lig, lig, lig, ost, o-z, o-z, o-z, irur, irur, irur, irur), Issan (l-o), issanagatic (o-z), issane (ost), issanic (bun, lig, irur), issaniké (bard), itzanik (lan), izaïn (garr), izaite (ahe), izak (lek, zur), izan (aiz, aiz, ahe, ahe, ahe, alo, alo, alo, ame, ame, arb, arb, arb, arr, arr, arr, arh, arh, arh, arh, ürr, ürr, ürr, ürr, a-s, azka, azka, aih, aih, aih, b-l, b-l, bar, bar, bar, bar, bas, bas, bas, bas, be-l, be-l, bid, bid, bid, bid, bid, bir, bir, lek, lek, lek, bes, bes, bes, d-s, d-s, d-s, buz, buz, buz, sarr, zib, zib, d-b, d-b, d-b, d-b, ünd, esk, esk, ezt, ezt, ezt, gam, gam, gar, gar, gar, get, get, hal, 
hal, hal, hal, hal, haz, haz, haz, haz, haz, haz, hoz, iho, iri, izp, jat, jat, jat, jat, jat, jats, lak, lak, lak, lak, lak, lak, laka, lekb, lekb, lekb, lekb, lah, lah, larz, lar, l-o, l-o, luk, luk, meh, meh, mit, mug, o-z, d-l, d-l, d-l, sar, sar, sar, sar, sar, sar, zur, zur, zur, dono, dono, bai, bai, bai, bai, dona, dom, dom, dom, dom, suh, suh, suh, suh, u-g, u-g, u-g, u-h, u-h, u-h, u-h, urk, urk, urk, uzt, uzt, uzt, mil, mil, mil, mil, mil, mil), izan) (meh), Izan (alo, arb, arr, a-s, gar, lak), izana (bar, ezt, iri, luk, ost), izanaatic (bes, larz), izanac (bid), izanagatic (be-l), izanagatik (l-o), izana_gatik (jats), Izancen (get), izandaila (azka), izanic (azka, get, meh, dono, dom), izanican (u-h), izanik (d-s, zur), izanikée (gam), izanike (garr), izanikere (aiz), izanique (bir), izaniqueré (ur-p), izantzen (aiz, azka, larz, mit), Izantzen (mil), Izantzenê (zur), izantzien (zur), izantzutenian (zur), izantzuzun (iho), izanzirenian (a-s), izate (d-l), izatu (bir, bir, get, ur-p), izatun (ur-p), izatuzuten (ur-p), nahiçan (arü, arü, altz, altz, altz, bard, larr, larr, larr, müs, müs, pag, ath, ure, ure, ure), nahisan (kan, l-s, l-o, l-o, mit), nahizan (urk), san (ünd), zan (sarr, ünd)

izen (1): icéna (lah)

izigarri (11): icigarrico (aint), icigari (hau), icigarri (laka, lig, larr, larr), icigarrico (zar, ainh), izigarri (bar, bar, esk)

izitu (3): icitcen (bida), isitsen (ban), izitzen (d-1)

izkina (1): ichkinean (uzt)

izotz (1): izotzarekin (sar)

jabe (4): yabeak (zib, d-l), yabiac (hal, jat)

jagi (1): yaguiteco (hir)

jaiki (1): yaiki (arn)

jainko (190): Goure-Yincoa (arm), Gure-Jincoua- (amo, ), Ginco (alt, alt, bil), Gincouaren (mar, pag, arro, bil), Jaincoaren (mug), (Jainco) (ame), Jaincoa (luh), Jaincoaren (a-s, ban, hoz, iho, izp, its, lak, lar, luh, hir, u-g, mil), Jainkoa (u-h, mil), Jainkoaren (d-l), Jinco (g-z), Jinco (ainh, esk, ezt, etxa, hau, lekb, lig, l-a, mug, müs, o-z, s-g, ath, z-p), Jincoa (d-i, z-p), Jincoén (ito), Jincoaren (d-s, d-i, dom), Jincouaïn (g-z), Jincouaën (hau), Jincouac (osp), Jincouaen (alo, altz, etx, l-z), Jincouaren (ainh, amo, arü, ürr, bel, kan, sarr, ünd, esk, etxa, g-i, ida, larr, l-a, mau, mit, müs, ora, urd, s-g, zal, ath, irur), Jincuan (bir), Jincuaren (soh), Jinküaren (d-b), Jinko (men), Jinkoäin (aiz), Jinkouaren (gar, laka, lig), Jinkouren (bar), Jinkuan (bas), Tjinncoärénn (hen), Yaïncoa (luk), Yaïncoaren (buz, luk, ure), Yaïnkoaren (haz), Yainco (ame, bard, lek, jut, ort), Yaincoa (iru), Yaincoac (hal, jat), Yaincoaren (ame, arm, azk, b-l, lek, ezt, get, iri, iru, izt, jut, las, mak, ort, sen, dono, urr, uzt), Yaincoua (lah), Yaincouain (mend), Yaincouaren (beh, bard), Yainco-Yauna (las), Yaincuaren (suh), Yainko (haz), Yainkoaren (ahe, bida, zib, esp, sar, u-h), Yainkouain (azka), Yeinkoaren (meh), Yenkoaren (l-o), Yinco (zar, bit, bid, l-s, leko), Yincoa (dom), Yincoac (aih, ur-p), Yincoaen (leko, d-g), Yincoain (a-zo, bid), Yincoarén (ain, l-s, ost), Yincoaren (anh, bit, lah, ald, dona), Yincoua (bun), Yincouaïn (a-z, iba), yincouac (bun), Yincouak (don), Yincouaren (zar, lekb), Yincuaan (aih), Yincuaïn (bai), Yinkhouaan (hel), Yinko (garr, zur), Yinkoa (arh), Yinkoaren (zur), Yinkouain (gam), Yinkouain (ilh, l-b), Yinkouarén (don), Yinkouaren (arh), Yinkua (bes), Yinkuain (gab, larz), Yinkuaren (jats), Yncoain (izu), Yncouaen (g-zo), Yncua (izu), Yuhkua (urk), Yunkuan (urk), Yainco (eih), Yaincoaren (aha, arn, eih)

Jainko Jauna (1): Yainco Yaouna (aint) 
jakin (145): iakitéco (ito), iakiteco (d-g), jakin (iho), jakinez (d-l), jakitéco (kan, etx, hau), jakitea (altz, osp, pag), jakiteco (ainh, alo, arh, ban, be-l, g-i, ida, lak, lar, l-z, mar, mit, mug, müs, arro, s-g, ath, u-g), jakiteko (arü, ürr, d-s, d-b, gar, ilh, izp, men, ora, mil, bil), jakitera (bar, ünd, esk, laka, lig, larr, urd, zal, irur), jaquitéco (g-z, o-z), jaquitéra (z-p), jaquiteco (bir, soh, etxa, mau), jaquitera (l-a, d-i), tjaquitéco (hen), yakin (zib), Yakin (aih), yakitéa (a-z), yakitèco (bun), yakitéco (ain, zar, lah), yakitéko (beh, don), yakitea (leko), yakiteco (azk, aih, bas, bid, bes, sarr, ezt, gab, get, hel, hal, haz, hoz, iri, izt, its, jut, lekb, larz, las, luk, meh, mend, ost, ort, izu, sen, dono, dona, u-h, urk), Yakiteco (bida), yakiteko (aiz, anh, arb, arr, a-s, azka, bard, esp, gam, garr, jats, l-b, lan, ald, d-l, sar, zur), yakitekotzat (zib), yakitera (b-l, lek, buz, jat, l-o, luh, mak, dom, suh), yaquin (l-s), yaquiteco (amo, a-zo, bit, g-zo, iru, l-s, bai, urr), yaquitera (ame, ure), yakitéco (aint), yakiteco (a-m, aha, arn, beho), yaquiterat (eih)

jakintsu (7): jakintsu (etx, l-z), jakintzu (d-s), yakinsun (mug), yakinsunik (zib), yakintsun (lan), yakintzu (garr)

jalgi (8): jalgi (iho), yalgui (bit), yali (bes), yalqui (iba), yelgui (ame), yelkhi (a-zo, g-zo), zaldiezin (garr)

jalki (3): jalkhi (g-z), jalki (mar), jelkhi (l-a)

jan (311): yatéa (aint), yatean (arn), yateat (a-m, aha), yaten (aha, beho, eih), yaterat (arn, beho, eih), yatiaïkin (a-m), yatian (aint), ïatéa (ito), gaten (bil), iatéa (ito), iatén (ito), iaten (d-g), jan (kan, iho, lar), janez (zib, ora, d-l), jatéa (g-z, etx, hau, o-z), jaténn (hen), jatéra (ainh, kan, kan, z-p), jatea (alo, arh, ürr, altz, d-b, d-b, ilh, osp, l-z, men, mend), jate'at (ban), jateat (iho), jaten (ainh, alt, arü, altz, ban, d-s, sarr, d-b, ünd, etxa, g-i, ida, izp, laka, lig, larr, osp, l-a, mar, mau, müs, urd, o-z, pag, s-g, d-i, irur, u-g, mil), jatera (alt, arh, arü, arü, bar, be-l, soh, sarr, ünd, esk, etxa, gar, g-i, ida, izp, laka, lig, larr, lar, l-a, mar, mau, mit, müs, ora, urd, pag, arro, s-g, zal, ath, irur, bil), jaterat (d-s, ezt, iho, lak, mug, u-g, mil), jates (ath), jatez (bar, be-l, soh, esk, etx, l-z, arro), jatiaëki (hau), jatiaeki (alo), jatiakin (ilh), jatian (arh, bir), jatiéqui (g-z), jatiareki (gar), jatiarekin (ürr, lak, mit), jatiarequi (zal, z-p), jatieaeki (men), tjaténn (hen), tjatérat (hen), yan (ahe, lek), Yan (jut, jut), yanez (bas, bes, izt, jats, mak, meh, mend, dona), yatéa (zar, a-z, iba), yatéat (beh), yatéco (ost), yatén (ain, l-s, don), yatènn (bit), yatéra (bit, bun, lah, don), yatérat (ain), yate (urk), yatea (lek, bes, buz, gam, hel, jats, jut, larz, l-s, leko, izu, u-h), yatearequin (ame), yateat (a-zo, bard, bas, bir, g-zo, gab, garr), yateco (haz, its, urr), yaten (amo, arb, a-zo, arr, arm, azk, azka, b-l, bid, bid, bida, buz, g-zo, esp, ezt, gam, garr, hel, hal, hoz, iba, iri, iru, its, jat, lan, las, ald, l-o, leko, mug, ort, sen, hir, sar, zur, bai, dom, suh, ure, ur-p, ur-p, urr, uzt), yatera (ahe, ahe, ame, amo, anh, arm, esp, hal, hal, izt, jat, jat, l-b, lekb, lan, las, l-o, luk, mak, d-i, sar, bai, dona, dom, suh, u-h, ure), yaterat (arb, arb, arr, arr, a-s, azk, azka, aih, b-l, b-l, bida, lek, zib, haz, hoz, iri, iru, ald, luh, luh, meh, ort, d-l, d-g, sen, hir, zur, dono), yatez (lekb), yathiarekin (aiz), yatia-akin (aih), yatiaikin (a-z, gab), yatiaékin (beh), yatiaikine (ost), yatiaikin (larz, izu), yatiaiquin (jut), yatiakin (bard, l-b), yatian (zar, anh, a-s, lek, bun, get, haz, dono, u-h, urk), yatiarekin (luk)

janari (1): yanarri (zib)

jarraiki (24): jaraikiic (l-z), jaraikiik (etx), jaraïkirik (ünd), jaraïquiten (o-z), jaraikirik (sarr), jaraikitzen (zib), jarraïkiac (pag), jarraikkic (hau), jarraikiric (lig, irur), jarraïkirik (gar), jarraïkirk (altz), jarraikiric (ürr, arro), jarraikirik (mit), jarraikitzen (ezt), jeraikiric 
(mau), jerraïkiric (ida), yaraïkiak (l-o), yaraïquic (l-s), yarraïkiac (sen), yarraïkirik (don), yarraikiak (leko), yarreikitzen (sar)

jarri (220): yaraaci (a-m), yarraaci (aha, aint), yarrarazi (beho), iarraaci (d-g, d-g), iarraraci (ito), iarri (hal, jat), iarricen (d-g), jar éassi(lig, o-z, irur), jar'aci (sarr), jar_éaci (alt, etx), jaraci (g-z, hau), jar_aci (ünd, men), jar-aci (arü), jar_araci (soh, iru, ora, ost), jaréraci (z-p), jararasi (kan), jar_arasi (arh), jar_arazi (iho, lak), jar_arrazi (izp), jar_ eaci (osp, l-z, pag, bil), jareraci (urd), jar_eraci (be-l, etxa, g-i, ida, larr, mar, mau, müs, arro, s-g), jar_erassi (mit), jar_erazi (alo, bar, esk, gar, zal), jar_errassi (ürr), jar_haraci (hen), jari (etx, ida, l-z, mar, mau, men), jarra'asi (ban), jarraazi (d-b), jarraci (altz), jarraraci (d-i), jarréraci (ainh), jarrarasten (lar), jarrarazi (d-s, u-g, mil), jarreraci (laka), jarri (arh, arü, ürr, altz, ban, be-1, soh, soh, sarr, gar, g-i, hau, izp, izp, laka, mit, pag, zal, zal, u-g), jarria (d-b), jarricen (ünd, ilh), jartcéa (ost), jartcen (iba), yar (arn, eih), yar azi (haz), yar erasi l-o), yar arasten (luh), yar_aasten (g-zo), yaraci (get), yar_araçi (bit), yararaci (azk, jut, sen), yar_araci (ain, bida, ost, dona, urr, uzt), yar_arasi (u-h), yar_arassi (mend), yar_arazi (ame, a-s, lah, bai), yar-arazi (luk, sar), yar-arazten (iri), yarassi (l-s), yareazi (urk), yar_eraci (amo, b-l, mak), yar_erazi (lekb, dom), yarhaaci (beh), yar_harasten (ald), yar_harazi (zib), yari (a-m, l-o), yarraaci (zar, iba, iba, las, leko), yarraasten (a-zo), yarraazi (bas, bes, gam, hel), yarraàzi (azka), yarraci (anh, bir, gab, ilh, l-b, izu, ur-p), Yarraci (arm), yarraraçi (ort), yarraraci (a-z, lek, esp, hoz, its, mug, hir, suh, ure), yarrarasi (izt), yarrarazi (arb, arr, aih, bid, buz, ezt, hal, jat, jats, meh, d-l, dono), yarraraztén (don), yarrarazten (ahe), yarrazi (garr, larz), yarrâzi (zur), yarrazten (lan), yarreci (bard), yarrhassi (bun), yarri (aha, aint, arn, eih, zar, amo, beh, bit, bun, ezt, gam, iru, jats, jut, l-b, las, ald, luk, mend, izu, u-h), yarria (buz), yarriçouçoun (arm), yarrizen (aiz, zur), yartsen (luh), yartzen (lan), yartzenda (azka), yar (arn, eih), yari (a-m), yarri (aha, aint, arn, eih)

jaun (110): Goue-Jaouna (l-z), Goue-Jauna (etx), GoureYauna (buz), Goure-Jaouna (o-z, gar), Goure-Jaüna (mit, sarr), Gure-Yaüna (bai), Gure-Yauna (uzt), Gure-Yaüna (gab), GureJauna- (hir), Gure-Jauna (hir), Yainco-Yauna (las), Yésous-Yaouna (bit), güre iaüna (dg), jaüna (hen), Jaüna (ürr, gam, hau, mug, s-g), jaouna (ath), Jaouna (bar, laka, lig, irur), jauna (ban, osp), Jauna (ainh, alo, arü, be-l, bir, d-s, soh, d-b, ezt, g-i, hoz, ida, iho, izp, its, larr, arro, d-l, zal, u-g, bil), Jauna- (mar), Jaunac (bas), Jaunan (mau), jouna (etxa), Yaüna (a-s, beh, bit, ünd, iba, leko, pag, ure), Yaüna- (jats), Yaünac (lekb), yaouna (a-zo), Yaouna (ain, bard, hel, l-b, ost, sen), Yaounac (dona), yauna (ahe, bida, iri, lan, d-l, hir, sar), Yauna (zar, ame, ame, anh, a-z, azka, b-l, g-zo, zib, get, hal, ilh, jat, jut, l-o, mak, mend, ort, zur, dono, ur-p, urr), yaunac (haz), Yaunac (bid, lek, ald, meh), yaunak (esp), Yaunaren (lan), Gure-Yauna (a-m), Yauna (aha, arn, beho)

Jaun Done (2): yandon (aha), Yandone (aha)

jautsi (2): jautsi (d-l), yauts (bida)

jauzi (1): yauzi (zib)

jeiki (76): yeiki (aint, beho), yeki (a-m), yekicen (aha), -jeiki (arü, hau), -yeïki (dona), -yeiki (leko), jaïki (etx, gar, l-z), jaiki (mar), jéiki (ath), jeiki (altz, lekb, larr), jeïqui (alt), jeiki (ainh, ürr, bar, be-l, soh, sarr, d-b, ünd, esk, g-i, ida, izp, laka, lig, lar, osp, men, müs, zal, irur, u-g), Jeiki (mit), jeiqui (g-z, etxa, l-a, o-z), jekicen (ilh), jeyki (pag), jeyqui (d-i, z-p), yaïki (aih), yaiki (b-l), yéiki (zar, iba), yékiten (beh), yeïki (bun, buz, hel), yeikitzen (gam), yeiki (ame, bid, bes, ezt, jut, las, ort), yeikitcen (g-zo, suh), 
yeikitzen (azka, l-o), yeiqui (l-s), yekhitzen (aiz), yeki (bard), yekicen (l-b), yekiten (a-zo), yekizen (zur), yequi (arb, iru), yeyki (urk)

jende (163): géndi (hen), genté (z-p), gentiac (alt, etxa, ida, larr, müs, urd, pag, zal), gentiak (ath), gentic (arü, arro), gentik (altz, bil), iéndiac (ito), iende (d-g), ienki (d-g), jéntiac (hau), jëntic (g-z), jende (amo, arh, ban, ban, bir, d-s, izp, lak, ora, d-i, u-g, mil), jendiac (iho), jendiak (d-b, jats), jente (aiz, bar, aiz, osp, mar, bil), Jente (ath), jentiac (ainh, be-l, soh, etx, g-i, lig, osp, l-z, l-a, mau, o-z, s-g, irur, z-p), jentiak (gar, laka), Jentiak (alo), jentic (ürr, ünd, esk, mit), jentik (bar, sarr, men), yèndé (bit), yéndé (ain, bun, ost, ost, don), yénde (iba), yendé ( $a-z, a-z$, beh, lah, ur-p), yende (ame, anh, arb, a-zo, arr, arm, arm, a-s, azk, azka, aih, b-l, bas, bid, bida, lek, lek, bes, g-zo, zib, zib, esp, ezt, gam, garr, get, hel, haz, haz, hoz, ilh, iri, iru, izt, its, jut, l-b, lekb, lan, larz, l-s, las, ald, luh, luk, mak, meh, leko, mend, mug, ort, ort, izu, sen, hir, sar, zur, dono, bai, dona, dom, suh, u-h, urk, ure, ure, urr, uzt), Yende (ahe, aih), yendea (d-l), Yendee (aih), yendiac (zar, bard, gab), yendiak (buz), yenté (kan), yentiac (l-o), yende (a-m, aha, arn, beho, eih), yendiac (aint)

Jesukristo (33): Chrichto (izt), J (urr), Jésou-Christo (kan, kan), Jésu-Cristo (ito, ito), JésusChrist (larr), Jesü-Krichto (d-b), Jessou-Christo-Goure-Jaouna (ora), Jesu-Chrichto (ban), Jesu-Christ (osp), Jesu-Christo (b-l, d-s, garr, izp), Jesukrist (g-i), JesuKristo (d-g), Jesu-Kristo (sar, u-g), Jesus-Christ (altz, irur), Jesus-Christo (suh), (Jesus-Crist) (arro), (Yésu-Cristo) (a-z), Yésukhrichto (esp), Yésus-Kristo (aiz), Yesuchristo (bida, bida), Yesu-Christo (ahe, ahe, g-zo, mak), Yesus-Christo (iri)

Jesus (147): Jésus (g-z, hau, its, larr, l-a, mau, men, o-z, ath, irur, z-p), Jesous (lekb), Jessous (ora), Jesu (bas, l-o), Jesus (alo, amo, arü, ürr, altz, aih, ban, bar, bas, be-l, bir, d-s, soh, sarr, d-b, esk, ezt, etx, etxa, gar, garr, g-i, hal, hoz, hoz, ida, iho, izp, jat, lak, laka, lig, lar, osp, l-z, l-o, mar, mit, mug, müs, urd, pag, arro, s-g, d-l, d-l, zal, dono, suh, u-g, uzt, uzt, bil), Jeus (arm), Jeuss (mil), Tjéçous (hen), Yéjouj (ost), Yéjus (iba), yésous (gam), Yésous (zar, arh, bun, lah), Yésous-Yaouna (bit), Yéssous (ain), yéssus (beh), Yéssus (bard), yésus (dom), Yésus (a-z, a-zo, esp, izt, izt, l-s, don), Yechuch (sen), Yesous (a-s, buz, hel, jut, ald, luh), Yessus (gab, lan), Yesu (anh, a-zo), Yesus (aiz, anh, azk, azka, b-l, bid, lek, bes, g-zo, zib, ünd, get, haz, ilh, iri, iru, jats, l-b, larz, las, luk, mak, meh, leko, mend, ort, izu, d-g, d-i, sar, zur, bai, dona, u-h, urk, ure, ur-p, urr), Yesus (arn), Jesu-Christo (arn), Yéchuch (aha), Yésous (eih), yésus (a-m), Yesus (aint, beho) jin (169): gïnen (pag), ginen (arü, a-s, etxa, l-a, mau, ort, bil, bil), ginendun) (pag), giten (etxa, zal, ath), jien (alt, d-b, ilh, o-z), jin (d-b, etxa, iho, izp), jinën (g-z), jinén (ito), Jinaracico (d-i), jinen (ainh, alo, ürr, altz, ban, ban, bar, be-l, be-l, d-s, kan, soh, sarr, ünd, esk, etx, gar, g-i, hau, ida, izp, lak, lak, laka, lig, lan, larr, lar, osp, l-z, l-o, mar, men, müs, ora, urd, o-z, arro, s-g, d-i, ath, irur, mil, z-p), jinenduc (u-g), jinic (zal), jintencien (ünd), jitén (hau), jiten (ainh, arü, ürr, ban, bar, be-l, soh, sarr, gar, g-i, ilh, laka, lig, mit, müs, urd, o-z, pag, irur, z-p), jitenciren (mit), jnen (arh), yéïnduc (a-z), yen (aiz, urk), yenen (its, d-g), yenenduk (esp), yieïn (gam), yien (a-zo, beh, hel, l-b, leko), yienduc (bes), yi-in (aih), yin (bard), yinac (a-z), yinén (l-s, ost, don), yinènn (bit), yinen (zar, amo, anh, arb, arr, azka, b-l, bas, lek, bun, buz, ezt, gab, hal, hoz, iri, iru, izt, jat, jats, jut, lekb, lekb, lah, larz, las, ald, luh, luk, mak, meh, dono, bai, dona, dom, suh, ure), yinenduk (haz, zur), yinene (bid), yiten (beh, iba, l-b), ynen (arm, arm, g-zo, izu, u-h), yten (lekb), yinen (a-m, aha, aint, arn, beho) 
Jinko (3): Yincouaren (aint), Yincuaren (a-m, beho)

jo (153): Go (mar), io (d-g), jo (alt, arh, arü, ürr, ban, ban, bar, be-l, kan, soh, sarr, d-b, ünd, esk, etx, gar, g-i, hau, iho, iho, izp, laka, larr, osp, l-a, mau, men, mit, urd, o-z, arro, s-g, d-i, zal, ath, u-g, bil, z-p), Jo (ainh, alo, altz, d-s, ezt, etxa, ito, lak, l-z, mil), joiten (müs), Joïten (lar), joiten (ida), jotzen (zib), tjo (hen), yo (ain, zar, ahe, ahe, amo, anh, a-z, azk, beh, b-l, bard, bit, bid, bir, lek, lek, bun, buz, g-zo, gab, garr, garr, get, hel, hoz, iba, iru, izt, its, jats, jut, lekb, lah, larz, l-s, las, ald, l-o, luk, mak, leko, mend, ora, ost, ort, izu, sen, sar, sar, bai, dona, suh, u-h, urk, ure, ur-p, urr, uzt), Yo (ame, arb, arr, arm, aih, bas, bes, hal, haz, jat, l-b, meh, mug, pag, d-l, dono, dom), yoac (hir), yoïten (gam, don), Yoçuten (ilh), yoeitin (a-zo), yoiten (lan), Yoiten (hir), yok (bida), yoka (ahe), Yotcen (esp), yoten (iri), yotsen (luh), yozouten (a-s), yozuten (azka, zur), Yozuten (aiz), yo (a-m, aha, aint, beho), Yo (arn, eih)

joan (467): yoan (arn, beho), youan (aha, aint, eih), youancen (beho), yuan (a-m, aint, beho), gan (azk, azk, azk, bida, bida, bida, bir, bir, zib, zib, get, get, ort, ort, d-l, d-l, ur-p, urp, ur-p), gançen (ur-p), gancen (ort), ganzen (get), goan (sen, sen, sen), guan (ahe, ahe, sar, sar, sar, urr, urr, urr), ioan (d-g), joan (anh, kan, kan, kan, lak, lak, lak, lar, mug, mug, ora, ora, u-g, mil, mil, z-p, z-p, z-p), johan (mil), jouaïten (be-l, lig, s-g, irur), jouaitenda (müs), jouaiten (soh, l-a, zal), jouan (alt, alt, alo, alo, arh, arh, arh, arü, ürr, ürr, be-l, be-l, be-l, bid, kan, g-z, g-z, g-z, soh, soh, soh, etx, etx, etx, etxa, gar, gar, gar, g-i, g-i, g-i, g-i, hau, ida, ida, ida, ilh, ito, ito, jut, laka, lig, lig, lig, larr, larr, larr, osp, osp, l-z, l-z, l-z, l-a, l-a, l-a, mar, mar, mar, mar, mau, mau, mau, mit, müs, müs, müs, ora, o-z, o-z, o-z, pag, pag, pag, arro, s-g, s-g, s-g, zal, zal, zal, ath, ath, ath, irur, irur, irur), jouann (hau, hau, hen), jouansén (ito), jouantsen (o-z), jouantzen (mit), jouiten (men, urd, arro), joun (ainh, ainh, ainh, arü, arü, ürr, altz, altz, altz, bar, bar, sarr, sarr, ünd, ünd, ünd, esk, esk, esk, etxa, etxa, etxa, laka, laka, men, men, men, mit, urd, urd, arro, arro, bil, bil, bil), juan (aiz, aiz, ban, ban, ban, d-s, d-s, d-s, d-s, d-b, d-b, iho, iho, iho, izp, izp, d-i, d-i, u-g, u-g), juana (d-b), juanahi (bir), juàtcénn (hen), jun (sarr), juten (garr), tjouänn (hen), uiancen (d-g), yoaiten (lah), yoaittenn (bit), yoaiten (leko), yoan (ain, ain, ain, anh, anh, anh, arb, arb, arr, arr, b-l, b-l, b-l, bas, bit, garr, hal, hal, haz, haz, izt, izt, its, jat, jat, lah, lah, lah, lah, l-o, l-o, l-o, mak, mak, meh, leko, leko, mug, izu, d-g, don, don, hir, hir, dom, dom, dom, urk, urk, ure, ure, ure), yoann (bit), yohan (hel, iru, luh, mug, zur), youaïten (bun), youaiten (dona), youan (zar, zar, zar, a-z, a-z, a-zo, a-zo, a-zo, arm, arm, a-s, a-s, a-s, beh, beh, beh, bard, bard, bard, bid, bid, bid, bun, bun, bun, buz, g-zo, g-zo, gam, gam, gam, garr, hel, hel, hel, iba, iba, iba, ilh, ilh, ilh, jut, jut, l-b, l-b, lekb, l-s, l-s, ald, dona, dona, dona), Youan (l-b), youana (arm, buz), Youanden (a-zo), youane (ost), youann (bit, g-zo), youantcen (l-b), youantzen (a-s), youanzen (a-s), youiten (beh), youn (l-s), youran (lekb, lekb, lekb), yuaiten (bas, lan, luh), yuan (ame, ame, ame, amo, amo, amo, a-z, azka, azka, aih, aih, aih, bas, lek, lek, bes, bes, bes, buz, esp, esp, ezt, ezt, ezt, gab, gab, gab, gab, haz, haz, hoz, hoz, hoz, iri, iri, iri, iru, iru, its, its, jats, jats, jats, lan, lan, larz, larz, las, las, las, luh, luh, luk, luk, luk, mak, meh, meh, leko, mend, mend, mend, ost, izu, izu, zur, dono, dono, dono, dono, bai, bai, bai, bai, suh, suh, suh, u-h, u-h, u-h, uzt, uzt), yuane (ost), yuantcen (esp, uzt), yuantzen (lan, larz, zur), yuanzen (azka, urk), yueiten (bard)

Jondone (8): yondané (a-m), Yondone (aint), Yondoni (aint, arn, eih) 
justizia (135): jousticia (kan), joustizia (arh), jujstisia (ban), justicia (ainh, alo, amo, arü, ürr, altz, be-l, bir, d-s, g-z, soh, sarr, ünd, etxa, g-i, hau, ida, ito, laka, lan, larr, osp, l-a, mar, mau, mit, müs, urd, o-z, pag, arro, s-g, d-i, hir, zal, ath, u-g, urr, uzt, bil, z-p), Justicia (alt, ilh), justissia (lig, irur), justizia (aiz, azka, bar, d-b, esk, gar, iho, izp, lak), juzticia (men), juztizia (mil), tjousticia (hen), youchticia (sen), youssticia (bit), yousticia (zar, iba, jut, dona), youstisia (lah), youstissia (bun), youstizia (buz, lekb), youztizia (gam), yujticia (ost), yusticia (ame, anh, a-z, a-zo, arm, azk, aih, beh, b-l, bard, bid, bida, lek, g-zo, esp, ezt, gab, get, hoz, iri, iru, izt, its, l-b, las, ald, luh, mak, meh, leko, mug, ora, ort, d-g, don, dom, suh, u-h, ure, ur-p), Yusticia (mend), yustician (izu), yustissia (l-s), yustizea (garr), yustizia (ahe, a-s, bas, haz, jats, larz, luk, sar, zur, dono, bai), yuztizia (urk), justicia (beho), yousticia (aha, aint, eih), yusticia (a-m, arn)

kabala beguirazale (1): kabale beguiraçale (bil)

kabala zaintzale (1): kabala zaintzale (d-s)

kabalzain (3): cabalé sagnés (o-z), cabalaçaignez (jut), cabalaçain (ora)

kanpo (3): campoan (lar), Kampoco (ame), Kampoko (d-l)

kanpotar (8): campotar (urr), campotiar (iho), campotiarrec (amo, luk), kampotar (l-o), kampotiarrak (bida), kampotiarrek (d-s)

kantoi (1): kantoïnean (luh)

kantu (4): khantiala (lig), khantuco (altz, o-z, o-z)

karitate (2): kaitatia (lig, irur)

karraska (2): carrascaz (izt), karraskan (zur)

karrika (13): carikara (luh), carricaï (alt), carricaco (alt), karikaren (meh), karrikaco (meh), karrikarat (meh), kharicaco (lig, irur), kharrica (arro), kharricala (arro), kharrika (esk, l-a), kharrikala (esk)

karroin (71): karroïn (aha), kharrogna (beho), kharroïn (aint, eih), kharroina (arn), kharroña (a-m), carrogna (bas), carrougna (beh, bun, ito), karoïna (lak, d-i), karroina (mend), karrogna (jats), karroina (ald), karrou (men), karrougna (l-b), kharroïn (zar, gam, don), kharroïna (buz, iru, las, d-g, suh), kharrogna (azka, ezt, iba), kharroiña (hoz), kharroigna (amo), kharroin (d-s, izp, jut, lan), kharroina (a-s, lekb, dona, u-g), kharrou (bar, larr), kharroua (ainh, altz, g-i, laka, osp, pag), kharrouak (ürr, mit), kharrouïnbat (garr), kharroubat (z-p), kharrougn (larz, ost, izu), kharrougna (a-z, a-zo, arh, bard, bit, etxa, ilh, ora), kharrouign (g-zo), kharrouk (arü, sarr, ünd), kharrounte (gar), kharruña (d-b), kharrugna (gab, luk, u-h)

karroin-aide (1): kharou aidiac (1-a)

karroin-beltz (11): karoumbat (mar), kharou (hau, ida, mau, müs, o-z), kharoua (lig, urd, bil), kharougna (1-s), kharouinbat (1-o)

karroindatu (12): kharountatcen (g-z, etx, l-z, zal), kharountatuic (irur), kharountatxen (ath), kharountatzen (alo), kharrountatcen (be-1, soh, arro, s-g), kharrountatzen (esk)

karrointe (1): carronté (alt)

kasatu (104): Casatiac (beho), cassatiac (aint), kasatiac (a-m), Khachatiac (aha), Khassatiac (eih), acassaturic (etxa), Acassaturic (mau), acazaturic (esk), akasaturik (sarr), akassaturic (arü), caçaturic (ida), cajsatiac (ban), Casatüic (g-z), casatiac (arh, aih, bid, iho, suh, u-h), Casatiac (jut, lak, ort, izu, d-g, d-i, dono, bai, dona, u-g), casatouyac (kan), casatuac (lek, iri, izt), Casatuac (bir, get, leko), casatuak (haz), casaturic (be-l, ezt), casatuyac (mug), Casatuyac (bas, bes), cassatiac (zar, z-p), Cassatiac (anh, a-z, 
ora, ure), Cassatouac (lah), cassatuac (azk), Cassatuac (its), cassaturic (ainh, soh, müs), Cassaturic (amo), Kajatiac (ost), kasatiac (lekb, luk, dom), Kasatiac (arm, hoz, larz), kasatiak (buz, jats, l-b, don), kasatouac (luh), kasatourik (a-s), Kasatuac (meh), kasatuak (ahe, esp, mil), kasatuik (men), kasaturic (las), kasaturik (ünd), Kasatuyac (arb), Kasatuyak (arr, zur), kasjatiac (iba), kassatiac (bit, gab), Kassatiac (lan), kassatiak (gam), Kassatiak (beh), kassatuic (a-zo), kassatuik (altz), Kassaturic (mar), Kassatuyac (mak), Kazatuac (ame), khasatiac (iru), Khasatiac (azka, ald), khasatiak (d-s), khasatuac (b-l, hel), khasatuik (urk), khassatatiak (bard), khassatiac (bun), Khassatiac (mend), Khassatiak (ilh), khassatuic (g-zo), khassutuic (l-s), khazatuik (garr)

kasko (1): cassko (bit)

kasu (3): khasu (bes), Cassou (eih), Khasu (a-m)

kasu egin (47): Cassu eguic (ida), cassu eguinçac (ure), cassu éguincéçac (z-p), Kasouiçac (arm), Kasouiç̧an (arm), Casu izak (lek), casu eisak (leko), casu egineçak (men), intzac casu (haz), casu eguizu (ezt), casu eguiten (izp), casu eguitin (izp), casu* eguinezac (jats), cazu eguitén (hau), kasou eguisan (luh), kasou eguiten (ald), kasou isac (ald), kasou eguinan (lekb), kasou eguic (lekb), kasu eiçak (esp), kasu egik (sar), kasu izak (zur), kasu izac (meh), kasu egin (ürr), kasu egitendu (d-g), kasu eguiten (laka), kazu eguiten (ath), khasou in (jut), khassou éguiten (a-s), Khassou éguissac (bun), Khassu egin (mit), khassu ein (gab, gab), khassu isac (l-s), khassu egik (l-o), khassu eintsak (bard), khassu egin (mit), eissac khasu (mug), eisan khasu (mug), eizak khasu (urk), eizan khasu (urk), khasu eguic (dom), khasu eguin (bil, bil), khasu egik (buz), Khasu egiten (u-g), kasu eguinzak (aiz)

kasu eman (7): emac cassu (lar), emaçue casu (its), Casumac (bid), kasu emac (iho), Kasu eman (arr), kasu emac (arb), Khasou man (hel)

kasuegile (1): kasoueguilerik (luh)

kausitu (128): causitu (arn, beho), causitzen (beho), kaouchitou (aha), khaouchitzen (aha), khaoussitou (aint), khausitcen (a-m, arn), khaussitcen (eih), khaüsitu (a-m), caüsitcen (meh, ort), caüsitu (gab, mug, mug, ort), caüsitzen (jats), caüssitcen (gab), caouchitu (ito), caoussitcén (ain), causicen (bir), causitcen (azk, izt, suh, urr, uzt), causitu (azk, bid, bir, lek, get, iho, izt, leko, uzt), causituçuten (hir), causitubaitciren (hir), causitzen (bas, bid, ezt, iho, iru), caussitcen (anh), caussitu (anh), caustu (urr), kaüsitcen (arb, arr, arm), kaüsitou (hen), kaüsitu (jats, meh), kaüsitzen (luk), kaüssitu (ure), kaöucitcénn (hen), kaoussitu (beh, l-b), kausitcen (esp, leko, dono), kausitou (arm), kausitsen (ald), kausitu (haz, sar), Kausitu (dono), kausitzen (ame, azka, d-b, sar, mil, mil), kaussitcen (mend), kaussitu (mend), kausten (esp), kauusitu (bida), khaüsitou (arh), khaüsitu (iru), khaüsitzen (arh, bai, dom), khaousitcen (dona), khaousitou (kan, dona), khaousitsen (kan), khaousitu (jut), khaousitzen (hel), khaoussitcen (bard, ora), khaoussitou (zar, lah), khaoussitzen (gam), khausitcen (hoz, las, mak, d-i, ure), khausitou (a-s), khausitsen (u-h), khausitu (b-l, bas, bes, d-s, buz, zib, gar, hoz, larz, las, mak, izu, d-i, dom, u-h), khausitzen (a-s, bes, buz, ezt, izp, lak, larz), khausjitcen (iba), khaussitcen (lar), khaussitu (bard, lan), khaussitzen (lah, lan), khautsicen (b-l), khauzitu (ame), khoussitzen (gam)

kendu (4): khendia (beho), kendiac (anh), khendiac (gab), khendu (u-g)

kexatu (1): khéchatiéz (ainh)

kolera (1): coléra (lah) 
koleratu (6): coleatiez (pag), coleratiez (izp, mend), coleratu (izu), coleratuz (bid), koleatiez (gam)

kondaira (7): condjiera (ure, ure), conyera (bai, bai), khondera (zib), kondera (ezt, ezt)

kontent (2): content (aint, zar)

kontrako (1): contraco (lan)

kontu (5): khountu (mit, mit, bil, bil), kountu (urd)

kontu egin (1): contou egui (sen)

kontu eman (5):contu eman (ur-p), Contu eman (get, urr), kontu emak (d-1), Kontu emak (bida)

kontutu (1): Kontutzen (d-1)

koro (1): khoro (garr)

kristo (5): Christ (altz), Christo (a-zo), Cristo (anh, bas), Krist (l-o)

kuinta (4): couïntac (amo), couintac (s-g), kuintak (d-l)

kukutu (34): cucutia (a-m, beho), coucoutia (a-z, arm, bit, bun, g-zo, iba, ito, jut, l-s, ora, ost, suh), coucoutuic (a-zo), cucutcen (d-g), cucutia (amo, aih, gab, d-g, u-g, u-h), kükütüik (d-b), koukoutia (arh, beh, buz, garr, ilh, l-b, izu), koukoutzen (aiz), kukutia (larz, dom),

kutu (1): coutsén (itu)

kunplitu (1): kompli (garr)

kurritu (1): courritu (garr)

kuxian (6): couchian (arr, sen), cuchian (arb), Cuchian (get), kuchean (ahe), kuchian (d-1)

laborari (144): laüari (d-b), laboaï (alt, altz, g-z, o-z), Laboaï (alo, etx, hau, ath), Laboaïbatec (irur), Laboai (l-z), laborari (ahe, ainh, a-z, arb, a-zo, arr, ürr, aih, ban, bar, bit, bid, bes, buz, kan, g-zo, sarr, esk, ezt, gab, gam, g-i, hal, hen, hoz, iho, izp, jat, l-b, laka, lekb, lig, larz, lar, las, luh, urd, ost, sen, don, sar, dono, bai, dom, ure, uzt, mil), Laborari (aiz, ain, zar, ame, amo, anh, arh, arm, azk, azka, beh, bas, be-l, bir, lek, bun, d-s, soh, esp, etxa, gar, garr, get, hel, haz, iba, ida, iru, izt, ito, its, jats, jut, lak, lah, lan, larr, l-s, ald, osp, l-a, l-o, luk, mak, mau, leko, men, mend, müs, ora, ort, izu, arro, s-g, d-i, hir, zal, zur, dona, suh, u-h, ur-p, bil), laborariatec (d-g), laboraribatec (arü, b-l, mit, z-p), Laboraribatec (mar, mug, pag), laboraribatek (ünd, u-g, urk), Laboraribatek (a-s), Laborary (urr), Labori (meh), laourari (bard), laouri (ilh), Laborari (a-m, aint, arn, beho, eih), Laboraribatek (aha)

lagundu (160): laüncen (arm, leko), laündu (d-b, ito, bai), laüntcen (g-zo), laüntzen (bai), lagüntu (g-z), lagoundou (bun, gam, hel, jut, lekb, luh, ora, sen, dona), Lagoundou (ain, arh, kan, hen, lah), lagounsen (bun), lagountcén (ain, don), lagountcénn (hen), lagountcen (jut), lagountsen (luh), lagountzen (buz, lah), lagun (lar), laguncen (alt, azk), lagundou (ald), lagundu (ame, anh, arü, azk, aih, beh, ban, bid, bida, lek, d-s, zib, esp, esk, ezt, etxa, gab, garr, get, hal, haz, hoz, iho, ilh, iri, iru, izp, izt, its, jat, jats, l-b, lak, lan, l-o, meh, mug, d-l, d-i, hir, sar, zur, dono, suh, ure, ur-p, urr, uzt), (lagundu) (luk), Lagundu (leko, ort, u-h, mil), lagungoua (bar), laguntcen (anh, arb, a-zo, arr, lek, esp, gab, hoz, izt, lar, osp, meh, ort, pag, hir, urr), laguntsén (kan), laguntsen (o-z), laguntu (alo, amo, ürr, altz, be-l, soh, sarr, ünd, hau, ida, laka, larr, mar, mau, mit, urd, o-z, pag, s-g, bil), Laguntu (men), laguntzen (ban, bas, bes, d-s, ezt, garr, hel, hal, haz, iri, jat, lak, las, l-o, luk, sar, mil), laoundou (zar, bard, iba, ost), laountcen (zar), laundou (mend), laundu (bir, izu), launtzen (aih), lagoundou (eih), 
Lagundu (arn), laguntcen (arn, eih), laoundou (aint), laountcen (aint)

laguntza (3): laguntza (zib, d-1, dom)

lak (5): lac (be-l, soh, etxa, ost, z-p)

Laku (9): laco (ito, las, osp, d-l, d-i), lacua (get), lacubat (urr), lako (d-b, gam)

landa (52): landa (zib, luh, urr), landac (ain, a-m, aha, eih arb, arr, arm, azk, aih, bas, bir, bes, buz, kan, get, hel, hen, iho, iru, izt, its, lak, lekb, lah, lar, mak, meh, mug, ort, sen, dono, dona, ur-p, uzt), Landac (lek, iri, hir, dom), landak (bida, esp, gam, d-l, sar, zur, urk, mil), Landak (leko), lander (d-g, u-g)

langile (2): languille (bida, d-l)

lantu (2): lanthuac (hal, jat)

laster (31): lachter (sen), laster (ain, a-z, a-z, arb, arr, azk, bas, bida, bir, bes, zib, esp, gar, get, haz, hen, hoz, iho, iho, iri, ito, osp, luk, mug, ora, urd, ort, d-i, ure), lazter (garr)

laster egin (1): laster-egin (d-1)

lasterka (12): lasterca (be-l, soh, hoz, izp, lan, lar, osp, osp, bai), lasterka (ahe, haz, l-o)

lasterkatu (8): lastercatiac (izu), lastercaturic (esk, g-i, s-g), lasterkaturik (alo, bar, bil), lasterkatuyak (zur)

lasto (1): lastuac (meh)

lehen (97): léhénago (kan, etx, hen, lah, ost), léhènn (bit), léhénoo (iba), léhen (alt), Léhen (1s), lénénago (bun), Lénénago (z-p), leheño (zur), lehen (beho, eih d-b, jut, mau, o-z), Lehen (bes), lehena'u (ban), lehenago (a-m, aiz, zar, ainh, alo, ame, anh, arb, arr, arh, arü, ürr, azk, azka, beh, bard, be-l, bid, g-z, soh, sarr, ünd, esk, gar, g-i, hal, hoz, ida, iru, jat, jats, l-b, laka, lekb, larz, larr, lar, las, l-z, l-a, l-o, luk, men, mit, müs, hir, dono, bai, dona, suh, ath, u-h, ur-p, uzt, mil, bil), lehen_ago (lig, zal, irur), Lehenago (bas, garr, d-g, dom, u-g), lehenôo (mug), Lehenau (leko), Leheneco (lek), lehengo (sen), Lehengo (luh, sar), leheno (aha izu, urk), lehenogo (d-i), lehenoo (bir, hel, mend)

leiho (145): léihora (don), leïhouan (aha, aint), léihouane (ost), léihoan (kan), léihouan (zar), léihoula (l-s), léyhora (bit), leïhiria (garr), leihoan (ain, arb, meh, leko, sen, dona), leïhoat (a-zo), leïhora (ito, lekb, bai), leïhouala (hau, lig, s-g, irur), leïhouan (a-z, arm, bun, buz, etx, gam, gar, l-z), leïhouann (hen), leïhoula (ainh, alt, altz), leïhuan (las), lehouan (mau), lehoun (ürr), leihoa (bida, bes, iba), leihoan (arn, arr, bid, iru, izt, lak, mug, ort, hir, u-g, ure, uzt), leihobat (mar), leihora (beho, b-l, ban, g-zo, ezt, gab, hal, hoz, iri, jat, lan, d-l), leihorat (ame, d-s, zib, iho), leihoratu (d-i), leihorric (iru), leihotic (aih), leihouala (g-i, osp, l-a), leihouan (amo, be-l, ida, ilh, jut, l-o, luh, izu, d-g, zal), leihouen (bard), leihoula (müs, urd, o-z), leihoun (esk, laka, men, mit), leihuan (d-b, get, izp, larz, luk, mend, dom, suh, u-h), leihun (urk), leinouan (l-b), leiuan (bir), leiyuan (ur-p), leyherat (lek), leyhoan (anh, haz, its, lar, mak, sar), leyhoat (bas), leyhora (ahe, a-s, azk, etxa, z-p), leyhorat (lah), leyhouala (g-z), leyhouan (arh, azka, beh, eih, soh, ora, pag), leyhoula (larr, ath, bil), leyhoun (arü, sarr, ünd), leyhuan (aiz, a-m, dono, mil), leyoan (esp), leyorat (ald, urr), leyuan (jats, zur)

leihoratu (3): leïhoualatu (alo), leihoulatu (arro), leihoulatzen (bar)

leize (5): léisebat (l-s), léissé (bit), leicebat (d-g, u-g), leize (azka)

leku (210): léchkian (ito), lékhïn (g-z), lékhian (aint, a-z, bun, l-s), lékhiane (ost), lékhou (ain, aint zar, hen, iba, ost, don), lékhu (a-z, etx, ito), léqu (alt), léquian (alt), lecu (aih), lehku (osp), lekhian (a-m, beho, eih, zar, alo, ame, arh, arm, azka, be-l, d-s, buz, soh, d-b, ezt, gar, g-i, hoz, ida, iho, ilh, iru, izp, l-b, lak, lekb, larz, larr, l-z, l-a, l-o, luk, 
mar, müs, ora, urd, o-z, izu, pag, s-g, zal, bai, dona, suh, ath, z-p), lekhiann (bit, hau), lekhin (ainh, arü, ürr, altz, bar, sarr, laka, mit, arro, bil), lekhou (aha, eih, arm, a-s, bit, bun, buz, gam, jut, lekb, ald, ora, dona), lekhouan (ain, sen), lekhu (aiz, ahe, ainh, alo, a-m, amo, arb, a-zo, arr, arü, beho, ürr, azka, beh, b-l, bar, bard, be-l, g-z, g-zo, soh, sarr, zib, d-b, ünd, esk, ezt, etxa, gar, g-i, hau, hoz, izp, l-b, lak, laka, lan, larz, larr, las, ald, l-z, luk, mak, mau, leko, mit, müs, urd, o-z, izu, pag, s-g, hir, sar, bai, dom, suh, ath, u-g, urk, uzt, bil, z-p, z-p), Lekhu (iho, sar), lekhuan (b-l, its, sar, u-g, u-h, uzt), lekhubatetan (alt), lekhuhartan (ilh), lekhuian (leko, d-g, dom), lekhuyan (arb, arr, bes, mug), lekian (aih, arn, ban, etx, jats, osp, dono), lekin (ünd, men), lekou (iru, luh), leku (aiz, anh, anh, bid, bir, lek, haz, izt, its, jats, men, mend, dono, ure, ur-p), Leku (lek, haz), lekuan (bida, haz, urr), lekuin (urk), lekuyan (esp)

lengoaia (1): lenguayan (bes)

listreatu (1): listreatcen (b-l)

lo egin (98): lo (arm, azk, kan, zib, hel, hal, jat, ald, luk, mar, leko, urd, sen, urk), loï (ost), loïc (aha, anh, a-z, a-zo, kan, g-zo, iba, ito, l-s, meh, mend, ora, dom), loïk (aiz, alo, bard, garr, l-b), loîk (zur), loic (bid, bir, bes, lan, izu, d-g, ur-p, z-p), loik (altz, d-b, hoz, ilh, larz, men, mil), lok (urk), loric (amo, arn, lek, get, hal, haz, iho, iri, izt, its, jat, lak, lah, las, ald, luh, mug, d-i, hir, dono, bai, suh, u-g), lorik (ahe, bida, d-s, zib, esp, ezt, don, sar), louac (esk, lig, osp, l-a, mau, arro, s-g, s-g, suh, bil), louak (alo, a-s, a-s, altz), louc (irur), lozelaik (garr)

loak hartu (27): lo hartu (zib), loac hartu (ainh, hal, jat, dom, ure, z-p), loac hartcen (mak, ure), loak hartu (mak), loac hartuc (d-i), loak hartcén (don), loc hartu (amo, izu, d-g, u-h), loc hartou (arh, bit, lekb), loc hartzen (arh, lekb), u-h), lohacartu (ur-p), loharcatzen (bas), loharkatu (bida), loharcatcen (hen), loharquatou (hen)

lokartu (111): lo arkhatzen (d-l), lo'khartcen (ban), locartcen (uzt), locartu (azk, aih, bid, bir), locartzen (aih), lokarcen (get), lokartcen (ort, urr), lokartu (anh, lek, haz, its, jats, luh, meh, mend, ort, d-l, urr), lokartua (dono), lokartucen (esp, leko), lokartuzen (aiz, zur), lokartzen (l-o), lokartzen) (luk), lokhartü (d-b), lokhartcén (ain), lokhartcen (a-m, aint, zar, arb, arr, beh, b-l, jut, lar, mar, dona), lokhartia (bard), lokhartou (zar, arm, bun, buz, hel, iba, jut, lah, ost, sen, dona), lokhartsen (bun), lokhartu (ain, ahe, a-m, ame, arn, a-z, arb, a-zo, arr, beh, b-l, ban, bas, bes, d-s, g-zo, ezt, gab, hoz, iho, ilh, iri, iru, izp, izt, lak, larz, las, luk, mug, ora, sar, bai, u-g, uzt), lokhartucen (l-b, hir), lokhartusén (l-s), lokhartuzen (azka, beho, mil), lokhartzen (ame, azka, buz, gab, gam, gam, iru, izp, jats, l-o), lokharzen (beho, lan), loc hartou (eih), lokartou (aint), lokhartoucen (aha)

losko (18): losco (ainh, arü, g-z, esk, arro), losko (ürr, sarr, ünd, gar, laka, mau, men, mit, urd, o-z, zal, bil), loskobat (ath)

lotsa (76): loçac (alt), lotça (alt), Lotaric (lah), lotcha (ito), lotsa (alo, bar, esk, etx, ida, laka, lig, l-s, l-z, men, mit, o-z, arro, s-g, irur, bil), lotsac (esk, etx, etxa, laka, l-z, o-z, arro, s-g, zal), lotsak (alo, altz, bar, men, bil), lotxa (ainh, etxa, larr, osp), lotxac (ainh, larr, osp, mit, müs, ath), lotxatuçu (ath), loxa (arü, ürr, altz, be-l, g-z, soh, sarr, ünd, gar, g-i, hau, l-a, müs, pag, zal, z-p), loxac (be-l, g-z, soh, g-i, hau, ida, l-a, mau, pag, z-p), loxak (arü, ürr, sarr, ünd, gar)

lotsatu (1): lotsatcén (ain)

lotu (58): lotcén (hau), lotcen (ainh, arü, be-l, soh, etx, etxa, g-i, ida, laka, larr, osp, l-z, mau, 
müs, pag, arro, zal, ath, bil), lothü (g-z), lothu (alt, arü, ürr, bar, be-l, soh, sarr, ünd, etx, etxa, gar, g-i, hau, ida, ito, laka, larr, l-z, men, mit, müs, urd, zal, ath), lothucen (pag), lotsën (g-z), lotsen (lig, irur), lotu (o-z), lotzen (ürr, bar, sarr, ünd, esk, gar, l-a, mit)

lur (445): loura (kan, kan, luh, luh), louréco (kan), loureco (luh), loureko (gam), lourra (aha, aint, eih, ain, ain, zar, zar, arh, arh, arm, arm, a-s, a-s, bit, bit, buz, buz, gam, gam, hel, hel, hen, iba, jut, lekb, lekb, lah, lah, ald, ald, ora, ora, ost, sen, sen, don, don, dona, dona), lourreco (aha, aint, eih), lourréco (ain, zar, bit, hen, iba, lah), lourreco (arm, hel, jut, ora, ost, sen, dona), lourreko (a-s, buz, ald), lourréko (don), Lur (bida), lur (zib, hal, jat, meh, d-l), lura (anh, azk, azk, g-z, g-z, etx, etx, gar, gar, hau, ida, ida, lak, lak, l-s, l-s, l-z, l-z, l-o, l-o, mar, mar, mau, müs, müs, o-z, o-z, d-i, d-i, zal, bil, bil, z-p, z-p), lureco (azk, g-z, osp, l-z, mau, müs, d-i), luréco (etx, l-s), lureko (alo, zib, gar, lak, l-o, d-g, bil), lurra (aiz, aiz, ahe, ahe, ainh, ainh, alt, alo, ame, ame, amo, amo, anh, a-z, a-z, arb, arb, a-zo, a-zo, arr, arr, arü, arü, ürr, ürr, azka, azka, altz, altz, aih, aih, beh, beh, b-l, ban, ban, bar, bar, bard, bard, bas, bas, be-l, be-l, bid, bid, bida, bida, bir, bir, lek, lek, bes, bes, bun, bun, d-s, d-s, g-zo, g-zo, soh, soh, sarr, sarr, zib, ünd, ünd, esp, esp, esk, esk, ezt, ezt, etxa, etxa, gab, gab, garr, g-i, g-i, get, get, hal, hal, haz, haz, hau, hen, hoz, hoz, iba, iho, ilh, ilh, iri, iri, iru, iru, izp, izp, izt, izt, ito, ito, its, its, jat, jat, jats, jats, jut, l-b, l-b, laka, laka, lig, lig, lan, lan, larz, larz, larr, larr, lar, lar, las, las, osp, osp, l-a, l-a, luk, luk, mak, mak, mau, meh, meh, leko, leko, men, men, mend, mend, mit, mit, mug, mug, urd, urd, ost, ort, izu, izu, pag, pag, arro, arro, s-g, s-g, d-l, d-g, d-g, hir, hir, sar, sar, zal, zur, zur, dono, dono, bai, bai, dom, dom, suh, suh, ath, ath, ath, irur, irur, u-g, u-g, u-h, u-h, urk, urk, ure, ure, ur-p, ur-p, urr, urr, uzt, uzt, mil, mil, a-m, aint, arn, beho, eih), lürra (d-b, d-b), lurrac (garr, lar), lurracen (ort), lurraïn (aiz), lurran (urk), lurrean (ame), lurreco (ainh, ame, amo, anh, a-zo, arh, altz, aih, b-l, ban, bas, be-l, bid, bir, bes, d-s, g-zo, soh, ünd, esk, ezt, etxa, gab, g-i, get, hal, hau, hoz, ida, iho, iri, iru, izp, izt, its, jat, laka, lig, lan, larz, larr, las, l-a, luk, mak, mar, leko, mug, urd, o-z, ort, izu, pag, arro, s-g, d-l, hir, zal, dono, bai, dom, suh, ath, irur, u-g, ure, urr, uzt, z-p, a-m, arn, beho), lurréco (bun, ito), lurreko (ahe, arb, arr, arü, ürr, azka, bar, bard, lek, sarr, garr, haz, ilh, jats, l-b, lekb, men, mend, mit, sar, zur, u-h, ur-p, mil), lurréko (a-z, beh), lürreko (d-b), lurrerat (zib), lurresko (esp)

lur-ikara (1): lur-ikarra (d-l)

lurlantzale (1): Lur lantzale (iri)

maite (2): maité (altz), maite (iru)

maiz (29): maïs (bir, kan, esp, get, hen), maïz (ahe, arb, arr, aih, bes, haz, lah, lar, mug, sen, ure), mais (mak, meh), maix (azk), maiz (bas, zib, hal, its, jat, d-l, hir, sar, zur, uzt)

malezia (4): maleciatiez (a-zo), malesian (ürr), malesiatiez (g-zo), malezian (mit)

malurus (85): malerosac (beho), malerouchac (aha), maléousac (l-s, o-z), maléousec (g-z), maléoussak (beh), maléouzac (hau), maléroujac (ost), malérousac (bun), malérousak (don), malérousec (z-p), malérousjac (iba), maléruzer (aiz), maléusec (alt), maleous (altz), maleousec (a-zo), maleoussak (gam), Maleoussak (ilh, l-b), maleoussec (gzo), malerousac (amo, larr, mar, müs), malerousac) (urd), malerousak (arü, sarr, ünd), malerousec (etxa, ida, mau), Malerousec (ainh, esk), malerousek (laka, men), malerouser (arro), maleroussac (soh), maleroussak (bard), malerouzak (mit), malerus (b-l), malerusac (a-m, luk, d-i), malerusak (ürr, mil, bil), malerussac (lan), maleusac 
(dom), maleusei (urk), malhourousa (ald), malhurosac (azka), Malhurosac (suh), malhurosec (hoz, jats), malhurusac (dono), Malhurusac (haz), malhurussec (gab), malhuruzac (ur-p), malorossac (anh), malouousac (hel), malour (a-s), malourosac (arm, dona), malourosec (jut), malourouchac (sen), malourousac (lekb), malouroussac (ora), malurosac (iho), Malurosac (bai, u-g), Malurosak (d-g), malurossac (mend), malurusac (aih, iru, izt, lak, mak), Malurusac (lek, get), malurusak (zur), malurussac (bid), malusac (bir), Maluusac (bes), maluusak (bas)

manera (3): maneran (be-l, soh, luk)

manu (3): mania (zal), maniac (irur), maniak (hoz)

markatu (1): markatcen (altz)

mehatxu (1): mehatchuz (iho)

memento (25): Mémènnto (bit), mémëntin (g-z), mémènto (bit), Mémentian (bun), Mémento (d-g), mémentouan (alt), mement (its), Mement (iru), mementian (larz), mementin (bar, esk, laka, arro), memento (ame, lekb, ath), Memento (ame, beho, lekb, u-g), mementouan (aha, lig, osp, zal, irur)

men (1): menian (bida)

mende (2): mende (hel), mendetan (lek)

mendi (157): Pyrénées-mendietako (sar, sar), méndi (ain, kan, hau, hen, l-s, ost, don, don, don), méndy (bun), mendïn (urd), mendi (aha, ahe, ainh, a-m, amo, arb, arm, arn, arr, a-s, a-s, a-z, azk, azka, aih, beh, beho, b -l, ban, bar, bard, bas, be-l, bid, bida, bir, lek, lek, bes, d-s, buz, eih, soh, zib, ezt, ezt, etxa, gam, hal, haz, hoz, hoz, iba, iho, iri, iru, izp, its, jat, jats, jut, lak, lekb, lah, lan, lan, larz, larz, larr, las, ald, osp, l-a, l-o, luh, luh, luk, mak, mau, meh, leko, men, mug, ost, ort, izu, pag, arro, s-g, d-l, d-i, sen, sar, zur, dono, dono, bai, dona, dom, suh, ath, urk, ure, ur-p, urr, uzt, mil), mendia (arm), mendiaïn (mend), mendian (aint, zar, alt, etx, haz, l-z), mendiaren (ame, get), mendibatean (lar, hir), mendibaten (anh, esp), mendibatetan (altz, mug), mendibatian (d-g, u-g, z-p), mendien (aiz, z-p), mendier (lan), mendietaco (bas, ald, luh, leko, leko, mug, hir, dom, dom), mendietako (esp, esp), menditaco (ezt, ezt), menditan (urk), mindi (a-zo, ürr, g-zo, gab, ilh, ito, l-b), mendiarte (aha)

mendiarte (3): mendiarté (a-z), mendiarte (mak, mend)

menditarte (2): menditarte (bard), mendi-tarte (sen)

menturatu (2): menturatcen (hoz), menturatzen (izp)

mestera (1): mestera (izp)

min (4): minac (hal, jat, hir), minak (ilh)

miragarri (1): miragarri (zib)

miragarriki (2): miragarriki (hal, jat)

miserable (1): miserabliac (larz)

miseria (2): miseriac (be-l, soh)

montaña (5): müntaña (d-b), müntañetako (d-b, d-b), montagnatetan (garr), mountagnabateen (ida)

moztu (27): mostiac (arh, u-h), moustia (mar), moustiak (ilh, l-b), moustu (ito), moustuic (hau, l-s, o-z), mousturic (arü, ünd, etxa, müs, z-p), mousturik (ürr, l-o), mouztuic (laka), mouzturic (sarr, esk, mit, zal), mouzturik (bar, men, bil), moztiac (a-z, buz), moztoua (ain)

muga (5): mougan (sen), mugan (ahe, bida, hal, jat) 
mulde (1): moulde (bar)

multzo (10): molcho (a-z), molchoat (aiz), moulço (arm), moultzo (ora), mulcho (luk), multçu (ure), multso (arh), (multso (ban), multsu (esp), multzo (u-h)

mutiko (142): Mithico (l-s), Mothico (ain, g-z, esk, etx, lak, laka, l-z, izu, irur), mothico (b-l, soh, g-i, hau, lekb, lig, osp, l-a, mau, mit, müs, o-z, pag, zal, dom, ure, z-p), Mothiko (alo), mothiko (ürr, altz, l-o, mar, ath, bil), Motico (anh), motico (kan), Motiko (aiz), mouchico (ida), Moutchiko (l-b), mouthico (aha, eih, a-zo, arh, arü, bit, bun, etxa, ito, jut, luh, ora, arro, s-g, dona), Mouthico (aint, zar, arm, g-zo, hel, iba, lah, ost), mouthikho (alt), mouthiko (a-s, sarr, ünd, gam), Mouthiko (bar, bard, buz, ilh, larr), Moutiko (ald), moutiko (men), mouttico (sen), Mouttiko (don), Muthico (ainh, aih, ban, bas, bes, ezt, iho, iri, izt, lan, las, urd, d-i, hir, bai, suh, u-g, arn), muthico (beho, be-l, get, hoz, iru, izp, larz, lar, luk, mak, mend, ort, u-h), Muthiko (ahe, arb, arr, d-g, sar, urk), Müthiko (d-b), muthiko (gar, mil), mutico (amo, azk, gab, urr, uzt), Mutico (bid, lek, leko, dono), Mutikho (azka), mutiko (ame, bida, esp, haz, its, jats, meh), Mutiko (beh, d-s, zib, d-l, zur), mutthico (a-m), Muttico (bir), muttico (mug)

mutikoño (1): Mouthicogno (a-z)

mutikotto (2): muthicotto (hal, jat)

mutil (2): mutil (hen), Mutil (ur-p)

muxi (1): mouchiic (osp)

naba (18): naba (ainh, alo, altz, bar, g-z, sarr, ünd, etx, gar, ito, larr, l-z, l-o, mau, müs, o-z, ath), nava (arü)

nabar (1): naarrian (aint)

nabaritu (2): nabaritu (hal, jat)

nagusi (115): naüsiac (amo, jats, meh, bai), naüsiak (ahe, mug), naüsiaz (d-b, ort), naüsik (sarr), naüssiac (arü, bun, gab, luk, mau, pag), naüssiaz (s-g), naüziak (aiz, garr), naöuçiac (hen), nagousiac (arm), nagousiaz (buz), nagusiac (get, iru), nagusiak (sar), nagussiac (its, ure), naouchiaz (sen), naoucic (ito), naoujiac (ost), naousia (altz), naousiac (alt, arh, kan), naousiak (don), naousias (l-s), naousiaz (gam, jut, l-a, dona), naousik (altz), naoussiac (ain, zar, a-z, a-zo, bit, lah, ora), naoussiak (azka, beh, bard, ilh, l-b), naujsias (ban), nausia (beho, bir), nausiac (a-m, arn, azk, aih, b-l, bas, bes, ezt, hoz, iho, iri, larz, luh, izu, d-g, d-i, zal, dom, suh, u-g, u-h, urr, uzt), nausiak (arb, bida, d-s, esp, leko), Nausiak (arr), nausiaz (bid, izt, lak, las, zur, dono, mil), nausiec (hir), nausik (ünd), nausiz (urk), nausjiétaz (iba), naussiac (aint, eih, etxa, lan, osp, mar, mend, urd), naussiak (l-o), naussiaz (anh, g-zo, hel, mak), naussiz (mit), nauziac (ame, ur-p), nauziak (a-s), nousiac (izp), naouchiac (aha)

nahi (608): emannay (ur-p), juanahi (bir), nahi (aha, ain, ain, aint, aiz, a-m, arn, zar, zar, ahe, ahe, ainh, ainh, alt, alt, alo, alo, ame, ame, amo, amo, anh, anh, a-z, a-z, arb, arb, a-zo, arr, arr, arh, arh, arm, arm, arü, ürr, ürr, a-s, a-s, azk, azk, azka, azka, aih, aih, beh, beh, b-l, b-l, ban, ban, bar, bar, bard, bard, bas, beho, be-l, be-l, bit, bit, bid, bid, bida, bir, eih, lek, lek, bes, bes, bun, bun, d-s, d-s, buz, buz, kan, kan, g-z, g-z, g-zo, g-zo, soh, soh, sarr, sarr, zib, zib, zib, d-b, d-b, ünd, ünd, esp, esp, esk, esk, ezt, ezt, etx, etxa, etxa, gab, gab, gam, gam, gar, garr, g-i, g-i, get, get, hel, hel, hal, hal, haz, hau, hau, hen, hen, hoz, hoz, iba, iba, ida, ida, iho, iho, ilh, ilh, iri, iri, iru, iru, izp, izp, izt, izt, ito, ito, its, its, jat, jat, jats, jats, jut, jut, l-b, l-b, lak, lak, laka, laka, lekb, lekb, lig, lig, lah, lah, lan, lan, larz, larz, larr, larr, lar, l-s, l-s, las, las, ald, ald, osp, osp, l-z, l-z, 
l-a, l-a, l-o, luh, luh, luk, luk, mak, mak, mar, mar, mau, mau, meh, meh, leko, leko, men, men, mend, mend, mit, mug, müs, ora, ora, urd, urd, ost, ost, o-z, ort, ort, izu, izu, pag, arro, arro, s-g, s-g, d-l, d-l, d-g, d-g, d-i, d-i, sen, sen, don, don, hir, hir, sar, zal, zal, zur, zur, dono, dono, bai, bai, dona, dona, dom, dom, suh, suh, ath, ath, irur, irur, u-g, u-g, u-h, urk, urk, ure, ur-p, urr, urr, uzt, uzt, mil, mil, bil, z-p, z-p), nahiçan (arü, altz, larr, müs, pag, ure), nahisan (l-o, mit), nahuken (mar), nai (bir, bir, bir), nay (ur-p, ur-p, aiz, aiz, ain, ain, ain, ain, zar, zar, ahe, ahe, ainh, ainh, alo, alo, ame, ame, amo, anh, anh, a-z, a-z, arb, arb, a-zo, a-zo, arr, arr, arh, arh, arm, arm, arü, ürr, ürr, ürr, a-s, a-s, a-s, azk, azk, azka, azka, altz, altz, aih, aih, beh, beh, beh, b-l, b-l, b-l, ban, ban, ban, bar, bard, bard, bas, bas, bas, be-l, be-l, bit, bid, bid, bida, bida, lek, lek, lek, bes, bes, bun, bun, d-s, d-s, d-s, buz, buz, kan, kan, kan, g-z, g-z, g-z, g-zo, g-zo, g-zo, soh, soh, soh, sarr, sarr, d-b, d-b, d-b, ünd, esp, esp, esp, esk, esk, ezt, ezt, ezt, ezt, etx, etx, etx, etxa, gab, gab, gab, gam, gam, gar, gar, gar, garr, garr, g-i, g-i, g-i, g-i, get, get, get, hel, hel, hel, hal, hal, hal, haz, haz, haz, haz, hau, hau, hau, hen, hen, hoz, hoz, iba, iba, ida, ida, iho, iho, ilh, ilh, iru, iru, iru, iru, izp, izp, izp, izt, izt, izt, izt, ito, ito, its, its, jat, jat, jat, jats, jats, jats, jut, jut, l-b, l-b, l-b, lak, lak, lak, laka, laka, lekb, lekb, lekb, lig, lig, lah, lah, lah, lan, lan, larz, larz, larz, larr, lar, lar, l-s, l-s, las, las, las, ald, ald, osp, osp, l-z, l-z, l-a, l-a, l-a, l-a, l-o, l-o, luk, luk, luk, mak, mak, mak, mak, mar, mar, mar, mau, mau, meh, meh, leko, leko, men, men, men, mend, mend, mit, mit, mug, mug, mug, müs, müs, ora, ora, ora, urd, urd, urd, urd, ost, ost, o-z, o-z, o-z, ort, ort, ort, izu, izu, izu, pag, arro, arro, s-g, s-g, d-l, d-g, d-g, d-i, sen, sen, sen, don, hir, hir, hir, hir, sar, sar, sar, sar, zal, zur, zur, zur, dono, dono, dono, bai, bai, dona, dona, dom, dom, suh, suh, suh, irur, irur, irur, u-g, u-g, u-h, u-h, urk, urk, urk, ure, ur-p, urr, urr, urr, uzt), uzt, uzt, mil, mil, mil, bil, bil, bil, z-p, z-p, z-p), nhai (ida)

nahi izan (17): nahiçan (arü, altz, altz, bard, larr, larr, müs, ath, ure, ure), nahisan (kan, l-s, 1-o), nahiukhen (laka), nahizan (urk), nahukhen (ünd, ath)

nahikatu (2): nahi catu (uzt), nahikatu (meh, d-l)

nahiz (9): nahis (arb, arr, esp), nahiz (ahe, hoz, iho, uzt), Nahiz (zib, luh)

nasa (1): nasa (izt)

nehor (84): néhoc (bit, ost, dona), Néhoque (iba), néhorc (bun, bun, lah, mug), néhork (don), neho'cke'e (ban), nehoc (a-m, aha, aint, zar, arm, azka, bas, bes, bes, hel, haz, haz, haz, hoz, iho, jut, bai), Nehoc (lak), nehok (d-s, buz, jats, leko, urk, mil), nehokée (gam), nehoke (mend), nehokec (izu), nehokee (bid, larz), nehorc (azka, b-l, b-l, lek, lek, iru, izp, izt, its, lekb, lan, lan, lar, ald, luh, ora, ora, ort, d-i, dono, suh, u-g, u-h, ure, urr, uzt, uzt), Nehorc (ame, beho, ezt, hal, its, jat, d-g), nehore (eih), nehorec (arh), Nehorek (aiz), nehork (bida, zib, zib, l-o), Nehork (bida), Nehorkec (las), nehorkek (bard)

nekatu (2): necatuac (ort), nekatiak (aiz)

neke (1): nekhian (aiz)

neska (26): néchca (ain), nechca (azk, bir, get, hal, its, jat, ur-p, uzt), nechka (ahe, arb, arr, bida, zib, haz, meh, sen, sar, zur), nechkabatec (lar), nesca (urr), neska (luh, d-l), netchéa (hen)

neskatila (20): nechcatila (ainh, zal), nechkatila (larr), nescatila (alt, soh, etx, hau, osp, l-z, l-a, mit, o-z, s-g), neskatila (alo, altz, mau, men, müs, ath), nezkatila (bard)

neskato (60): néchcato (iba), néchcatoché (ito, l-s), néchkato (bit, ost, don), nechcato (a-m, 
aint, anh, arm, ban, bas, beho, bid, ezt, hoz, iho, iri, iru, izp, izt, jut, las, luk, leko, ora, izu, d-i, bai, suh), nechkato (aha, zar, azka, buz, esp, gam, jats, lan, larz, ald, mend, ort, dona), neichcato (eih), nescato (b-l, lak, mak, mug, pag, d-g, dom, u-g, u-h), neskato (arh, a-s, aih, lek, bun, d-s, lekb)

neskatoko (4): nechkatoco (bes), nescatoco (lah), neskatoko (mil), nexkatoko (urk)

neskatuxe (16): neskateuche (ilh), neskhateuché (l-b), nechcatoché (a-zo), nechcatoco (hel), nechcatuchégno (a-z), nekatoche (l-o), nescatoche (amo, g-zo, etxa), neskatoce (dono), neskatoché (beh), neskatoche (d-b, mar), nezkatoche (aiz), nechkatoche (gab)

neskatxa (6): néscatcha (kan), nechcatcha (ure), nescatcha (esk, hir), neskatcha (ame), neskatchar (bar)

neskatxu (9): nescatchou (arro), nescatchouna (gar), nescatchun (be-l), neskachou (ürr), neskatchou ( $g-z)$, neskatchouna (arü, sarr, bil), neskat-chouna (ünd)

ni (1): ni (d-b)

nihor (46): nihük (d-b), nihoc (arb, arr), Nihoc (luk), nihor (bida), nihorc (ain, amo, anh, azk, kan, kan, hen, iru, mak, meh, sen, hir), Nihorc (aih, get, iri, meh, hir, dom), nihorec (arn), nihork (ahe, ahe, a-s, sar), Nihork (esp, d-1), nihorkec (mar), nihouc (a-z), nihouké (ito), nihoukéek (beh), nihoukec (gab), nihoukek (mar), nihourc (gzo), Nihourc (mau), nihourcek (urd), nihourek (garr, l-b), Nihourek (ilh), nihourkec (men), nihourq (a-zo), nihourqué (l-s), nihun (iru)

ñimiño (27): ñimiñobat (u-g), ñmiño (d-s), gnimigno (beh, bard, buz, gab, gab, gab, garr, haz, haz, haz, haz, mar, ora, ora), gnimignobat (ilh, l-b, mar, ora, d-g), gnimignobatek (ilh, ilh, l-b, l-b), gninignoatek (bard), nimino (lek)

no (ka) (2): no (aint, zar)

noiz (256): noïs (eih, kan, lar, ost, u-h, u-h), Noïs (aha, arh, eih, kan, mar), noïz (ain, a-m, arh, arh, beh, bit, lek, bun, bun, buz, eih, soh, gam, haz, hen, lekb, lah, lah, las, mug, sen, don, ure, ure), Noïz (zar, bit, bun, bun, gam, gam, haz, lekb, mug, sen, sen, dona, ure, ure), nois (esp, mak, mend), Nois (amo, amo, esp, esp, mak, mar, u-h, u-h), noiz (aha, aint, arn, beho, zar, ahe, ame, anh, anh, a-s, azk, ban, bas, bida, d-s, zib, zib, ezt, gab, gab, hal, hoz, iba, iru, izp, izt, izt, its, jat, jats, jats, jut, jut, lan, lan, larz, ald, l-o, mar, meh, müs, ora, ort, izu, d-i, sar, dona, dom, dom, urk, urr, mil, z-p), Noiz (a-m, aint, beho, ame, arh, a-s, a-s, azk, b-l, b-l, bida, bida, gab, gab, hal, hoz, iru, izp, its, jat, jats, jats, jut, jut, larz, larz, l-o, luk, meh, ora, ora, ort, dona, dom, dom, urr, mil, z-p), Noizee (mend), noizeta (arm, b-l), Noizeta (hoz), noiz-eta-ere (hir), nouis (g-z, etxa, pag), Nouïs (hau, o-z), nouïz (ünd), Nouiz (ünd, gar, gar), nouis (alt, hau, hau, ida, mau, o-z, s-g, ath, ath, bil), Nouis (alt, hau, l-a, l-a, mau, mau, o-z, pag, s-g, ath, ath), nouiz (ainh, a-zo, arü, ürr, altz, altz, bar, bard, be-l, be-l, g-zo, soh, sarr, esk, etx, gar, gar, g-i, laka, lig, lig, larr, osp, l-z, l-a, l-a, mit, urd, arro, arro, zal, irur, irur), Nouiz (arü, ürr, ürr, altz, altz, bar, be-l, be-l, soh, sarr, etxa, g-i, g-i, ida, ida, lig, mau, mit, mit, müs, müs, arro, arro, s-g, zal, bil, bil), Nouize (men, men), nouizee (men), nous (alt), Nuiz (soh), nuizee (men)

noiz ere (4): nouis é (hau, hau, hau), nouis é (hau)

noiz eta ere (1): nouiz eta é (altz)

noizbait (1): nouizpait (arro)

nola (2): nola (hoz), Nola (iho)

non (4): non (a-z, zib), noun (altz, lek) 
nonbait (3): nonbait (iri), nonbaitik (d-1), nonbeitik (zib)

nondik (2): Nondik (ahe), nountik (garr)

nor (143): nor (aha, ain, a-m, aint, arn, beho, eih, zar, ahe, ame, amo, anh, arb, arr, arh, arm, a-s, azk, azka, aih, beh, b-l, ban, bas, bit, bid, bida, bir, lek, bes, bun, d-s, buz, kan, zib, d-b, esp, ezt, garr, hel, hal, haz, hen, hoz, iri, iru, its, jat, jats, jut, l-b, lak, lekb, lah, lan, las, ald, l-o, luh, luk, mak, meh, leko, mend, ora, ost, ort, izu, d-l, d-g, d-i, sen, don, hir, sar, dono, bai, dona, dom, suh, u-h, urk, ure, ur-p, urr, uzt, mil, z-p), Nor (izp, mug), Nordiren (lar), nortciren (izt), nortzien (gam, iho, larz, zur), nour (ainh, alt, a-z, a-zo, arü, ürr, altz, bar, be-l, g-z, g-zo, soh, sarr, ünd, esk, etx, etxa, gar, hau, ida, ilh, laka, lig, larr, l-s, osp, l-z, l-a, l-o, mar, mau, men, mit, müs, urd, o-z, pag, arro, s-g, zal, ath, irur, bil), Nour (alo, g-i), nurtcien (gab)

nora (148): no'at (ban), noat (aha, a-m, aint, zar, a-z, arh, arm, beh, bas, bid, bir, bes, bun, d-b, gab, gam, garr, hel, iba, iho, ilh, ito, l-b, leko, mend, ost, izu, d-g, u-h, urk, ur-p), noât (zur), nora (lan, ure), norat (aiz, ain, ahe, ame, anh, arb, arn, arr, a-s, azk, azka, aih, beho, b-l, bida, eih, lek, d-s, buz, kan, soh, zib, esp, ezt, get, hal, haz, hen, hoz, iri, iru, izp, izt, its, jat, jats, jut, lak, lekb, lah, larz, lar, las, ald, luh, luk, mak, meh, mug, ora, ort, d-l, d-i, sen, don, hir, sar, dono, bai, dona, dom, suh, u-g, urr, uzt, mil, z-p), noua (hau), nouat (ainh, alt, alo, a-zo, arü, altz, bard, g-z, g-zo, sarr, ünd, etx, gar, g-i, lig, l-s, osp, l-z, l-a, mau, men, o-z, pag, ath, irur), nourat (amo, ürr, bar, be-l, bit, esk, etxa, ida, laka, larr, l-o, mar, mit, müs, urd, arro, s-g, zal, bil)

nork (4): norc (ito, u-g), norcien (iba), norciren (get)

obedient (1): obédient (altz)

obeditu (99): obéditou (arh, arm, a-s, eih, bit, bun, kan, hen, ost, don), obéditu (ahe, ainh, aint, aiz, b-l, hau, iru, arro, ur-p, z-p), obéitou (zar, iba), obeditcen (aha), obeïtou (gam, hel), obeïtu (bas, meh), obeditou (buz, jut, lekb, ald, luh, sen, dona), obeditu (anh, arb, arn, arr, ürr, azk, azka, aih, ban, lek, sarr, zib, ünd, esp, etxa, get, haz, ida, izp, izt, its, jats, lak, lan, larz, larr, las, l-a, l-o, luk, mar, mau, mend, mit, mug, müs, ora, izu, pag, d-l, d-g, hir, dono, suh, u-g, ure, urr, uzt, mil, bil), obeditzen (u-h), obeitu (a-m, amo, beho, bid, bir, bes, gab, iho, mak, leko, zur, bai, dom)

oberen (1): obeena (aha)

ofreitu (1): offfreitu (ame)

ogi (148): Oïak (bai), ogi (haz), Ogi (urk), ogiac (arn, be-l, hoz, u-h), Ogiac (aih, lek, bes, buz, haz, iho, ito, lak, lekb, d-g, u-g, mil), ogiak (ürr, gar, mit), Ogiak (d-s, zib, d-b, l-b, l-o, d-l, sar, zur), Ogic (sarr, esk), ogik (bar, men), Ogik (arü, ünd), ogui (iru), Ogui (lah), oguia (iba), Oguia (ezt, ur-p), oguiac (aint, beho, zar, alt, amo, arh, b-l, bun, soh, gab, g-i, hau, ida, jut, larz, l-a, mar, mau, leko, müs, ora, o-z, sen, dona, dom, ath, ure, urr), Oguiac (aha, ain, ainh, a-m, ame, anh, a-z, a-zo, arm, azk, azka, ban, bas, bit, bid, bir, eih, kan, g-z, g-zo, etx, etxa, get, hel, hal, hen, iba, iri, iru, izp, izt, its, jat, laka, lig, lan, larr, l-s, las, ald, osp, l-z, luh, luk, mak, mend, mug, urd, ost, ort, izu, pag, d-i, hir, zal, dono, suh, irur, uzt, z-p), oguiak (a-s, bida, esp, gam, garr, jats), Oguiak (aiz, ahe, alo, arb, arr, beh, bard, ilh, don), oguic (arro), oguik (altz, bil)

ohartu (25): ohartcen (get, lan), ohartou (eih, lah, ora, sen, dona), ohartoucen (aha), ohartouzen (hel), ohartu (a-m, arn, beh, esp, hoz, iho, izp, izt, its, lak, larz, luk, ort, dono, urr), ohartuzuzun (bes)

ohi (1): ohi (ahe) 
ohildu (14): ohilduac (d-l), ohilduak (zib), Ohiltiac (pag), ohiltuac (l-o), ohiltuik (urd), ohilturic (larr, zal), Ohilturic (ürr, lig, s-g, irur), Ohilturik (alo, gar, mit)

ohoin (149): ühüñek (d-b), ühünak (d-b), ogognac (hen, ur-p), ohoïnac (osp), ohoïnac (ain, anh, arm, buz, kan, get, hel, iru, jats, lekb, lah, lar, las, ald, luk, meh, mend, mug, sen, bai, suh, ure), Ohoïnac (lek), ohoïnak (aiz, arb, arr, leko, don), Ohoïnak (haz), ohognac (amo, ezt, iba, larz, ora, ost, izu, dona), ohognak (aha, gam), ohoiñac (zp), ohoiñak (zib), ohoignac (jut), ohoinac (arn, beho, zar, ame, azk, azka, b-l, ban, bid, bir, bes, hal, iho, iri, izt, its, jat, lak, lan, l-o, mar, d-g, d-i, dono, dom, u-g, urr, uzt), Ohoinac (aih, hoz, izp, luh), ohoinak (ahe, bas, bida, esp, mak, d-l, hir, sar, zur, urk, mil), Ohouïnac (bun), Ohoynak (d-s), ouhouñac (esk, urd), ouhouñak (ürr, bar), ouhougna (lig, irur), ouhougnac (ainh, a-z, a-zo, arh, bit, g-z, g-zo, etx, etxa, gab, g-i, hau, ida, l-z, l-a, mau, müs, o-z, pag, arro, s-g, zal, ath), Ouhougnac (alt, l-s), ouhougnéc (ito), ouhougnak (alo, altz, bard, gar, ilh, men, mit, bil), Ouhougnak (garr), ouhougnat (l-b), (Ouhougnétaï) (a-z), ouhouiñak (sarr, ünd), ouhouignac (beh, be-l, soh, laka), ouhouinak (arü, larr), ouhouynak (a-s), uhugnac (u-h), ohoïnac (a-m, eih), oihanac (ort)

ohointza (2): ohointçaric (lar), ohoyntzerik (d-s)

oihan (440): oïhan (aint), oihanetarat (arn), oihanetaric (arn), oïanialat (etx), oïhanaï (hel, mug), oïhanéako (bun), oïhanéat (l-s), oïhanéco (zar), oïhanérat (ain, don), oïhanari (gab), oïhanétan (ain, aint, zar, iba, ost), oïhénétan (ito), oïhanétann (bit), oïhanétic (ain, aint, bun, iba, lah), oïhanétik (don), oïhaneat (aha, aiz, a-m, bas, iba, mend, izu), oïhaneco (arm, iru, lah, las, ost, bai), oïhaneko (gam, ora), oïhanera (lekb), oïhanerat (arb, arr, buz, sen, suh), oïhanetan (aha, aiz, alo, a-m, amo, arü, altz, bas, bun, buz, sarr, ünd, gam, gar, garr, hel, ida, iru, laka, lekb, lig, l-z, mend, mit, mug, müs, urd, sen, bai, irur), oïhaneti (zar, altz), oïhanetic (aha, a-m, arü, etx, gab, lekb, lig, l-s, l-z, müs, ora, urd, ost, o-z, izu, s-g, sen, zal, bai, suh, ath, u-g), oïhanetik (arb, arr, buz, sarr, ünd, gam, gar, mit), oïhanialat (alo, laka, lig, l-a, irur), oïhanian (arb, don), oïhanilako (gar), oïhanilat (ainh, arü, altz, sarr, ünd, müs, urd, ath), oïhenari (bard), oïheneat (garr), oïhenetalat (a-zo), oïhenetan (a-zo, gab, l-b), oïhenetik (l-b), ohanetik (mend), oihaénétan (etx), oihan (izt), oihanari (bida, ida, lan, l-o), oihanétan (lah, l-s, z-p), oihane'at (ban), oihaneat (urk), oihaneco (beho, bir, bes, hal, iho, jat, lak, larz, luh, luk, ort, d-g, d-i, sar, u-g, uzt), oihaneko (zib, d-l), oihaneraco (izt), oihanerat (aih, b-l, bid, hoz, iri, izp, dom), oihanetan (arn, ürr, b-l, bar, bid, bida, bir, bes, zib, esk, g-i, hal, hoz, iho, iri, izp, izt, jat, lak, lan, larz, osp, l-a, l-o, luh, mau, men, o-z, ort, izu, d-l, d-g, d-i, sar, dom, suh, u-g, urk, uzt), oihaneti (l-a), oihanetic (aih, ban, bas, bid, bir, bes, esk, g-i, hel, hal, hoz, iho, iri, iru, izp, izt, jat, lak, lan, larz, larr, osp, mau, ort, d-g, d-i, dono, dom, z-p), oihanetik (alo, beho, ürr, bida, laka, l-o, luh, men, d-l, sar, urk), oihanetik" (bar, zib), oihanialat (g-i, osp, mau, zal), oihanian (arr, aih), oihanilat (ürr, bar, esk, men, mit), oiheneat (d-b, ilh, l-b), oihenetaïc (a-zo), oihenetan (d-b, ilh), oihenetik (d-b, ilh), oyénéko (beh), oyaneko (esp), oyanen (ur-p), oyanetan (esp, ald), oyanetarat (ald), oyanetik (esp, ald), oyanila (alt), oyenetan (ur-p), oyhan (its, meh), oyhanéco (z-p), oyhanérat (kan), oyhénarat (ito), oyhanari (ahe, anh, lek, d-s, mil), oyhénari (a-z), oyhanétan (ainh, kan, g-z, hau, larr), oyhénétan (a-z), oyhanéti (g-z), oyhanétic (alt, bit, eih, hau), oyhénétic (a-z, ito), oyhénétik (beh), oyhanbatétara (bit), oyhanean (meh), oyhaneco (eih, ezt, get, jut, leko, dona, ure, urr), oyhaneko (azka, 
haz, jats, zur), oyhanerat (amo, arh, a-s, azk, mak, mar, pag, hir, dono, u-h), oyhanetan (ahe, ame, anh, arh, arm, a-s, azka, beh, ban, beho, be-l, eih, lek, d-s, soh, ezt, get, haz, its, jats, jut, lar, las, luk, mak, mar, leko, ora, pag, arro, s-g, hir, hir, zal, zur, dono, dona, ath, u-h, ure, urr, mil, bil), oyhanetarat (ame), oyhaneti (pag), oyhanetic (ainh, ame, amo, anh, arh, arm, azk, azka, be-l, lek, kan, soh, ezt, get, haz, ida, its, jats, jut, lar, las, luk, mak, mar, meh, arro, hir, dona, irur, u-h, ure, urr), oyhanetic" (arm), oyhanetik (aiz, ahe, a-s, d-s, leko, zur, mil, bil), oyhanialat (be-l, g-z, soh, hau, l-z, s-g), oyhanian (azk, lar), oyhanilat (larr, o-z, arro), oyhann (bit), oyhanpétan (alt), oyheneco (etxa), oyhenetaic (g-zo), oyhenetalat (g-zo), oyhenetan (bard, g-zo, etxa), oyhenetic (bard, etxa), oyhenilat (bil)

oihanetu (1): oyanetu* (ur-p)

oihu (8): oïhu (ahe), oïhuen (l-o), oihik (bar), oihu (hal, ilh, jat), oyhu (hir), oyuac (ald) oihu egin (113): oihou itéco (aint), oihu egiteco (beho), huyu éguiteco (ur-p), oïhou eguiteco (arh), oïhou éguiteco (arm), oïhou éguitèco (bun), oïhou éguitéco (ost), Oihou éguiten (lah), oïhou éguitén (don), oïhou itéco (zar), oïhou iten (zar), Oïhou iten (aint), oïhuiten (ost), oyheguiten (irur), Oyhéguitën (g-z), Oihuïten (iba), oïhuteco (l-b), oyhuguiten (larr), oïhu eguiten (arr), oïhu eyteko (bas), Oïhu eguiten (arb), Oïhu eguinen (mil), oïhu iten (izu), oïhu eguiteco (a-m, suh), oïhu eguinen (suh), oïhu eguiteco (las), oïhu éguitéco (a-z), Oỉhu éguiten (a-z), oïhu iteco (a-zo), oïhu inen (a-zo), oïhu iteco (g-zo), oïhu inen (g-zo), oïhu iteco (gab), Oỉhu iten (gab), oïhu iten (garr), oïhu éïtia (ito), oïhu itéco (l-s), oïhu itén (l-s), Oïhu eguiten (ora), oïhu egiteco (u-h), Oỉhu egiten (uh), oïhu éguiteco (lig), oïhu eguiteco (irur), Oîhu iten (l-b), oïhy éguiteco (mug), Oihü iten (d-b), oihou eguiten (luh), oihou eguinen (luh), oihou iteco (jut), oihu éguinen (mug), oihu eiteko (urk), Oihu eiten (urk, urk), oihu eiteko (bard), oihu eiten (bard), Oihu egiten (sar), oihu éguiteco (dom), oihu eguiten (dom), oihu eitea (bes), Oihu eiten (bes), Oihu egiten (d-l), oihu eguiten (lar), oihu eguinen (uzt), oihu eguiteco (hoz), Oihu egiten (hoz), oihu iteco (iho), oihu egiten (iho), oihu eguiten (iri), oihu eguinen (lan), Oihu iten (larz), oihu iteco (izu), Oihu iten (azka), oihu eguiteco (ban), oihu eguiten (ban), Oihu eguinen (ald), oihu egiten (d-s), Oihu eguinen (ezt), oihu eguiteco (izp), Oihu eguiten (izp), oihu egitéco (d-g), oihu egiten (d-g), oihu egiten (u-g), oihu eguiteco (ame), oihu iteko (ilh), Oihu iten (ilh), oihu eguiteco (mar), Oihu eguiten (l-a), oihu éguiten ( $z-p)$, oyhou eguiten (arh), oyhou eguiteco (a-s), Oyhou eguiten (a-s), oyhou iteko (bit), oyhou éguinènn (bit), oyhu eguiten (hir), oyhu egiteko (zur), oyhu itendiat (zur), oyhu eguiteco (lek), oyhu eguiten (lek), oyhu egiteko (haz), Oyhu egiten (haz), oyhu eguiteco (azk), Oyhu eguiten (azk), Oyhu eguiten (get), oyhu eguiteco (arro), oyhu eguiten (arro), oyhu eyteco (d-s), oyhu eguiteco (jats), Oyhu eguinen (ame), oyhu itéko (beh), Oyhu iten (beh), oyhu itén (ito), oyhu eguitéco (g-z), oyhu éguitéco (z-p), oyou egiterat (ald)

oldartu (7): oldartiez (a-m, d-b, s-g, bai), oldartu (hoz, iru), oldartuyez (bas)

olha (1): olhan (don)

omen (2): Omena (its, its)

on (271): hün (d-b), hon (arh, ure), Hona (aiz, arh, bid, iru, ald, ure), honac (bid, iru, ald), honak (aiz), houn (ainh, alt, alo, arü, ürr, a-s, altz, be-l, bun, g-z, soh, sarr, ünd, esk, etx, etxa, gar, garr, g-i, hau, ida, ito, laka, lekb, lig, larr, l-s, l-z, l-a, l-o, mau, men, mit, müs, urd, o-z, izu, pag, arro, s-g, zal, ath, irur, bil, z-p), Houn (alt, alo, arü, ürr, bar, 
be-l, g-z, soh, sarr, etx, etxa, gar, g-i, hau, laka, lig, larr, l-z, l-a, mau, men, mit, müs, o-z, pag, s-g, zal, ath, irur, bil, z-p), houna (alt, alo, altz, bit, g-z, esk, esk, etx, garr, ida, ito, l-s, l-s, osp, osp, l-z, l-a, mau, urd), Houna (ainh, alt, alt, amo, a-z, arm, bun, buz, gam, ilh, jut, lekb, l-a, l-o, mar, ora, ost, izu, don, dona), hounaïn (garr), hounac (zar, amo, a-zo, arm, g-zo, iba, iho, jut, osp, mar, mend, ora, don, dona), Hounada (a-s, l-b), hounaen (men, o-z), hounak (bar, beh, bit, buz, gam, ilh, l-b, müs), hounaou (beh), Hounaouçou (g-zo), Hounaouçu (a-zo), hounaren (z-p), Hound (ünd), houne (ost), houneco (zal), hun (larz, luk, suh, u-h), Hun (ida), hunaüçu (gab), Hunaouçou (iba), Hunauzu (iho), on (bes, lah), ona (eih, zar, ahe, azk, aih, zib, luh, hir), (ona) (bida), Ona (ain, ame, arb, arn, arr, b-l, bas, bir, kan, esp, get, hel, hal, hen, iri, izt, its, jat, lak, lah, mak, meh, leko, ort, d-l, d-i, hir, sar, dono, suh, urk, urr, uzt, mil), onac (ain, ame, arb, arn, arr, azk, aih, b-l, bas, bir, lek, kan, get, hel, hal, haz, hen, iri, izt, its, jat, lak, lar, luh, meh, mug, ort, d-i, dono, dom, ur-p, urr, uzt), Onada (zur, ur-p), onak (ahe, zib, esp, mak, leko, d-l, sar, zur, urk, mil), (onak) (bida), Onauzu (bes), onecoa (sen), onecoac (sen), onekoa (bida), onekoak (bida), onena (ur-p), Ouna (bard), ounac (bard)

on-behar (2): on-beharrac (hal, jat)

ondasun (16): hontarçunic (z-p), hountarçounic (larr), hountarçunen (zal), hountarzun (alo), hountarzunen (bar), hountasun (d-g), huntarsun (u-g), ontansuntçat (b-l), ontasun (bida, zib, haz), ontasunac (lek), ontasunen (sar), ontazun (ame), Ontazunik (garr), ountasunen (1-o)

ondo (77): ondouan (aha), bort'ondoko (bar, bar), ondo (aint, arn, arro, beho, get, hen, ora, aiz, arm, a-s, bard, bit, get, hen, ito, jut, ora, arro, d-g), ondoan (aiz, ain, ahe, ahe, iri, ald, d-l, don), ondoco (ame, g-zo, lan), ondoko (altz, a-m), ondorat (arb, arr), ondoti (zar, ito), ondotic (aint, alt, hal, iho, iri, jat, lar, mar, hir, uzt), ondotik (arb, arn, arr, laka, mug, o-z, d-l, urk), ondotiq (etxa), ondouan (larr, l-a, l-o), ondouetaco (g-zo, arro, s-g, s-g), ondoun (bar, arro), onduan (urr), oundo (ilh, l-b), oundoco (a-zo, l-a), oundotik (l-a), oundouan (bun, garr, ito), oundouetaco (a-zo)

onetsi (1): onhexi (iri)

ongi (5): ongi (d-l), ongui (aiz, aiz, ahe, b-l)

ontsa (8): ontsa (iho), ontza (aiz), onza (garr), ountza (garr), ounxa (mar), ounxachko (l-a), untsa (lan), untxa (ban)

orain (30): oaï (ito), Oaï (l-s), oaïco (bas), Oagnic (bir), oai (d-b), oïno (aiz, bas), oîno (zur, zur), Oîno (zur), ohono (beh), Ora (ur-p), oraï (eih, haz, lar, lar), Oraï (garr), orai (ainh, lek, d-s, larr, luh, urk), Orai (iho), Oraiéé (beh), Oraico (azk, lek, luh), oray (kan), orayere (ur-p)

oraino (377): ôano (mug), ôno (ban, ilh, ilh, l-b, bai, bai), Oaïno (bas, ito), oagno (bir), oano (a-zo, arü, arü, altz, altz, altz, bun, buz, g-zo, g-zo, g-zo, sarr, etx, etx, etx, hau, hau, ito, lig, l-s, l-z, l-z, l-z, l-a, men, men, mug, o-z, o-z, pag, pag, ath, ath, irur, irur, urk), Oano (alt, alo, g-z, gar, hau, men, o-z, urk), ono (aha, zar, zar, ban, ban, bard, buz, buz, garr, ito, l-b), Ono (aha, arm, azka, garr, ilh, l-b), oogno (bir), oono (a-m, aint, a-z, a-z, a-z, azka, azka, aih, beh, bid, bid, bid, bes, bes, bes, d-b, d-b, d-b, gab, gab, gam, gam, hel, hel, iba, iba, iba, iho, jut, jut, larz, larz, leko, leko, leko, mend, mend, ost, ost, izu, izu, izu), Oono (aih, a-m, bes, gab, hel, jut, mend, ost), Oonoré (aint, zar), oonoré (aint), oonore (a-m), oraïno (ain, ain, ain, ahe, arb, arb, arr, arr, arh, bas, be-l, 
be-l, eih, kan, kan, kan, soh, soh, haz, haz, hen, hen, hen, jats, jats, lekb, lekb, lekb, lah, lah, lar, l-s, luk, meh, meh, ora, ora, sen, sen, don, don, dona, dona, ure, ure, ure), oraîno (anh), Oraïno (ain, anh, arh, bun, eih, jats, lah, lar, luk, ora, dona), Oraïno* (iru), Oraïnoko (haz), Oraïnon (meh), oraiño (zib, zib), oraigno (bida), oraino (ahe, ame, ame, ame, arm, arn, a-s, azk, beho, b-l, b-l, b-l, be-l, lek, lek, d-s, esp, esp, esp, ezt, ezt, get, get, hoz, hoz, hoz, iho, iri, iri, iri, iru, iru, izp, izp, izp, izt, izt, its, its, lak, lan, larz, lar, las, las, las, l-o, mak, mak, mar, mar, mar, ort, ort, d-l, d-l, d-g, d-i, d-i, sar, sar, dono, dono, dom, dom, dom, suh, suh, suh, u-g, u-h, urk, urr, urr, urr, uzt, uzt, mil, mil), Oraino (bida, d-s, ezt, get, izt, its, lan, ald, mak, ort, d-i, don, hir, hir, sar, u-h, uzt, mil), Orainon (dono), orano (ainh, ainh, ainh, alo, amo, amo, arü, ürr, ürr, bar, bar, g-z, soh, sarr, esk, esk, esk, etxa, gar, gar, g-i, g-i, g-i, ida, lak, laka, laka, lig, larr, osp, osp, l-a, l-a, l-o, l-o, mau, mau, mit, mit, müs, müs, urd, urd, arro, arro, s-g, s-g, s-g, zal, ath, irur, bil, bil, bil, z-p, z-p), Orano (amo, ürr, etxa, ida, lak, laka, lig, larr, osp, mau, mit, müs, pag, arro, zal, z-p), orayno (a-s), Orayno (a-s), orono (bit), Orono (bit, d-g, u-g), uano (alo)

ordain (1): ordainez (bid)

ordari (1): ordari (urd)

orde (9): orde (a-zo, a-s, g-zo, zib, gab, iri, lar, d-1), ordetan (aiz)

ordoki (18): ordoki (a-m, aint, arn, zar, bid, bun, d-s, d-b, iho, jats, jut, lak, osp, mar, leko, ort, izu), (ordokiak) (bida)

ordoki-arte (1): ordokhiarte (meh)

ordu (18): ordouz (aint), Ordou_berian (buz), ordu (zib, zib), Ordu (arh, zib, hal, hoz, its, jat, larz, meh, d-l, d-i, sar, u-h, urk, mil)

orduan (158): ordain (zal), Ordain (aiz), ordin (g-z), ordian (aha, aint, aiz, a-m, arn, beho, eih, zar, alo, amo, a-z, a-z, a-zo, arh, arm, azka, aih, beh, ban, be-l, bid, bun, d-s, buz, g-zo, soh, ezt, etx, gab, gam, gar, g-i, hau, hoz, iba, ida, iho, ilh, iru, izp, jats, jut, l-b, lak, laka, lig, larz, larr, l-s, las, ald, osp, l-z, l-a, l-o, luk, mar, mau, meh, mend, müs, ora, o-z, izu, s-g, d-g, d-i, don, dono, bai, dona, dom, suh, ath, irur, u-g, u-h, z-p), Ordian (aint, zar, alt, azka, aih, bid, d-b, garr, ito), ordiane (ost), ordiann (bit), ordien (bard), ordin (ainh, arü, ürr, altz, bar, sarr, ünd, esk, etxa, men, mit, urd, pag, arro, bil), ordouan (ain, a-s, kan, luh, sen), ordouann (hen), ordouian (lah), orduan (ahe, ame, azk, b-l, bida, bir, lek, esp, hel, haz, izt, its, lan, lar, leko, ort, d-l, hir, sar, ure, ur-p, urr, uzt, mil), Orduan (ahe, bir, zib, hal, haz, iri, jat, hir), Orduian (dom), orduin (urk), orduyan (arb, arr, bas, bes, mak, mug, zur), Orduyan (mug)

ordukotz (1): Orducotz (aih)

oro (316): aldootaïc (pag), o (hau), oö (o-z), Oôtaric (a-zo), ootaïc (etx, l-z, ost), Ootaïc (a-m, zar, g-zo, hel), ootaïk (leko, ath), Ootaik (altz, bard), Ootaik (urk), orataric (get), oro (a-m, aha, aint, arn, beho, zar, zar, ahe, ainh, ainh, ainh, alt, alo, alo, alo, ame, amo, a-z, a-z, arb, a-zo, a-zo, arr, arü, arü, arü, ürr, ürr, ürr, a-s, altz, altz, aih, beh, beh, beh, bar, bar, bard, bard, be-l, be-l, be-l, bit, bit, bun, buz, buz, g-z, g-z, g-z, g-zo, g-zo, soh, soh, soh, sarr, sarr, sarr, d-b, d-b, d-b, ünd, ünd, ünd, esk, esk, esk, ezt, ezt, etx, etx, etx, etxa, etxa, etxa, gab, gab, gab, gar, gar, gar, garr, g-i, g-i, g-i, g-i, hau, hau, hoz, iba, iba, ida, ida, ida, ida, iho, ilh, ilh, iri, izp, izp, ito, ito, jats, jats, jats, jut, l-b, laka, laka, laka, lig, lig, lan, lan, larz, larr, larr, l-s, l-s, osp, osp, osp, l-z, l-z, l-z, l-a, l-a, l-a, l-o, l-o, luk, luk, mau, mau, mau, mau, men, men, men, mend, mend, mend, mit, 
mit, mit, mug, müs, müs, müs, urd, urd, ost, ost, o-z, ort, pag, pag, pag, arro, arro, arro, s-g, s-g, s-g, d-g, don, hir, zal, zal, zal, zal, zur, dono, bai, suh, suh, ath, ath, ath, irur, irur, irur, u-g, uzt, uzt, mil, mil, mil, bil, bil, bil, z-p, z-p, z-p), Oro (d-s), oroekin (lig), oroidekiak (l-b), orotaïc (g-z, mend, d-g, bai), Orotaïc (bit, bun, buz, gab, ito, l-s), orotaïk (beh, garr), orotaîk (zur), Orotaïk (gam, garr, jats), orotaic (aha, bes, l-b, lan, ora), Orotaic (aih, aint), orotaik (azka, d-b, ilh, men), Orotaik (laka), orotan (arm), Orotan (l-a), orotaric (amo, anh, arr, arü, eih, ürr, sarr, ünd, gar, g-i, hal, hoz, jat, jut, lak, lig, lah, mak, mar, mau, mit, ort, s-g, dono, dona, irur, u-g, ure, bil), Orotaric (ainh, arh, arn, be-l, soh, esk, ezt, ida, iru, izp, izt, larr, las, osp, luk, mug, müs, urd, o-z, arro, hir, zal, dom, suh, u-h, uzt), orotarik (alo, arb, larz, luh), Orotarik (a-s, bar, l-o, mil), Orotariq (etxa), Orotzas (z-p), otaïc (hau), Otaïc (alt)

orobat (15): , oorat (ost), orobat (amo, arb, arr, ezt, lah, s-g, urr), Orobat (hal, hen, jat, larz, sen, suh)

osin (5): osin (iho), ossin (bard, müs, pag), oussi (its)

osoki (6): osoki (beho), osoki (gar, lak, dona), ossoki (ure), ossoqui (ure)

ostatatu (1): ostatatu (urd)

oste (1): oste (urr)

ote (3): othe (hal, jat, d-1)

ozar (1): oçar (ort)

paratu (4): pharatu (bida, bida, iho, meh)

pareko (1): pareco (ame)

pareta (1): pareta (d-s)

paropia (5): paropia (irur), Paropia (irur), paropiabatétan (irur), paropiaen (irur), paropiaren (irur)

parte (1): parteat (bir)

partitu (1): parthitoussiren (bun)

pasaiant (2): pasayant (ezt, izt)

pasatu (81): paastu (bid), pachatou (aha), pachatu (sen), pajsatu (ban), pasatcen (esp, d-g, urr), pasatou (kan, ald, luh, dona), pasatsen (luh, luh), pasatu (beho, bir, lek, get, haz, hoz, iru, jats, leko, ort, izu, don, dono, dom, u-g, uzt, mil), pasatuzen (zur, ur-p), passatcen (mend), passatou (arh, eih, hen, lah, ora), passatoussén (bun), passatouzen (gam), passatu (aint, anh, beh, gab, its, lak, l-s, mak, suh, u-h, ure), passatucen (mend), pazatu (ame), pazatucen (ur-p), phajatou (ost), phasatcen (lar), phasatou (buz, hel, jut, lekb), phasatouzen (a-s), phasatu (amo, bas, ezt, izt, larz, las, mug, urk), phasatuzen (azka, bes), phasatzen (lekb, urk), phasjatu (iba), phassatou (zar), phassatu (b-l, bard), phassatuic (lan), phassatzen (lan), phazatzentzen (garr)

pausa (1): paüchan (haz)

pena (2): phenac (lig, irur)

peña (7): pegna (azka, ban, bid), peigna (iru), phegna (arh, u-h, ure)

pentsatu (1): Penzatzen (garr)

pentze (97): pénsiac (bun), péntsiac (bit), peintciac (anh, bid, ora), peintziac (luk), penceac (arm, ort), penciac (arü, uzt), pensiac (mend), pentceac (mug, ure), pentciac (its, dom), pentciak (beh, esp, esp), pentsiac (kan, luh), pentzeac (ame, arn, iru, meh), pentzeak (ahe, mil), pentziac (aih, ban, lah, dono), pentziak (aiz, haz, jats, l-o), Pentziak (garr), penxer (d-g), penziak (a-s), phéntciac (ost), pheintziak (d-b), phenceak (leko), 
phencer (u-g), phenciac (azka, lek, lak, lan, mak), phensiac (arh, u-h), phentceac (izt, lar), phentçiac (aint), phentciac (a-m, eih, zar, jut, las, dona), phentciak (iba, suh), phentxiac (d-i), phentzeak (iri), phentzer (izp), phentziac (bas, bas, beho, buz, hel, hal, hoz, iho, jat, lekb, larz, bai), phentziak (aha, ezt, gam), phentzic (bes), phenziak (d-s), phinciac (a-zo, g-zo), phinsiak (bard), phintceac (b-l), phintciac (amo, a-z, etxa, gab, izu), phintciak (ilh, l-b), phintsiac (l-s), pinceri (ald), pinciac (ito, mar, pag), pintciak (don)

persegitu (26): perjéitiac (ost), persébituric (ainh), perséguituïc (g-z), persegitiac (lak), persegitiak (azka), persegituac (bid, meh), persegituak (sar), persegituik (men), perseguitiac (izp, ora, d-i, dona, z-p), perseguitiak (gam), perseguitourik (a-s), perseguituac (get, its), perseguituric (be-l, soh, müs, urd), persekeitiac (larz), persituic (a-zo), pertseytuïac (bas), perzéguitiak (beh)

Petri (260): Péthi (ath), Pétiri (beh, lah, o-z, don, don), Pétiriékin (beh), Pétiriéqui- (o-z), Pétirikin (mug), Pétri (zar, a-z, bit, bun, hen, ito, ost), Pétriékin (aint, zar), Pétriékin(ain), Pétriekin (bas), Pétriikin- (hel), Pétrirékin (bun), Pétrirékin- (a-z), Pedro (d-g), Pethiri (etx, larr, l-a), Pethirieki (gar), Pethirieqi- (l-a), Pethirireki (s-g), Pethiry (alt), Pethri (arh), Petiri (aih, bir, lek, d-b, garr, hal, haz, jat, las, ald, luk, men, mit, mug, zur, dono), Petiriekin (men), Petiriekin- (ald), Petiriikin (d-b), Petiri-ikin- (aih), Petirikin (aiz, lek, haz, zur), Petirikin- (dono), Petirikin) (garr), Petirirekin (buz, hal, lah, las, luk), Petirirekin) (jat), Petiri-dé-Commingesco (mug), Petrîquin (bai), Petri (aha, ahe, aint, a-m, a-zo, arm, arn, azka, ban, bas, bit, d-s, buz, eih, ezt, gam, hoz, iho, iri, izp, its, jut, lak, larz, meh, mend, ort, izu, hir, u-h, urk, ure), Petri'ek'in (ban), Petrie (azo), Petriekin (aha, a-zo, arh, d-g), Petriekin- (u-h), Petriekin) (bida), Petriequin (d-i), Petriikin (azka, iba, larz, izu), Petriikin- (bid, bes, esp), Petriikine (ost), Petrikin (gam, lan), Petrikin- (anh, gab, urk), Petriquin (bir), Pétrirekin (a-m), Petrirekin (ahe, arn, arr, azk, b-l, d-s, ezt, hoz, jats, jut, lak, meh, d-l, dona), Petrirekin- (arb, arm, iho, iri, izp, its, ort, sen, sar, u-g, uzt), Petrirekin) (ame, luh), Petrirequi (eih, ure), Petrirequin (get, hen), Petrirequin- (iru, hir, suh), Petrirequin) (urr), Phéti (hau), Phétiëki (hau), Phétiri (kan, kan, soh, z-p), Phétiriékin- (ainh), Phétirieki (lig), Phétiriequi (z-p), Phétiry (alt), Pheti (mau), Phetieki- (alo, ath), Phetiieki (l-z), Phetiieki- (etx), Phetirekin (lekb), Phetirequi (mau), Phetiri (alo, arü, ürr, bar, be-l, sarr, sarr, ünd, ünd, esk, etxa, gar, g-i, ida, ilh, l-b, laka, osp, osp, l-z, l-o, urd, pag, pag, arro, s-g, zal, irur), Phetirieki (arü, esk, müs, irur), Phetirieki- (g-i, mit, urd), Phetirieki) (larr), Phetiriekin (l-o), Phetiriequi (be-l), Phetiriequi- (soh), Phetirikin- (ilh, l-b), Phetiriquin (etxa), Phetirireki (bar, ida, zal), Phetirireki- (arro), Phetirirekin (ürr, laka), Phetiry (müs), Phetri (g-zo, g-zo, lekb),_Pétri (ain),_Petri (bes, esp, bai, dona),_Petri (ame, anh, arb, arr, azk, b-l, bid, bida, gab, get, hel, iba, iru, lan, luh, mend, d-l, d-i, sen, sar, suh, u-g, urr, uzt), -Petri (ur-p), -Petriquin" (ur-p),_Petri (jats), -Petri (ora), -Petrirekin (ora)

pidaiant (2): pidayant (aha), pirayant (eih)

Pierres (5): Piarres (zib), Piarresekin (zib), Pierres (aiz, mil), Pierresekin (mil)

Pierra (25): Piéraéqui- (g-z), Piera (g-z), Pierra (amo, altz, mar bil, bil), Pierré (bard), Pierraeki (altz), Pierraekin (mar), Pierrarequi- (amo), Pierre (a-s), Pierrekin (bard), Pierrekin- (as), Simpiéra (l-s), Simpiéaïquin- (l-s), Pierra (bil), Pierra ekin (bil), Pierraekin (mar), Pierrekin (bard), Pierré (bard), Pierre (beho), Pierrekin (beho)

pikatu (99): phïcatia (a-zo), phicatia (g-zo, ure), phicatiac (a-m, aint, eih, zar, arm, hoz, iba, iho, 
jut, lak, luk, ost, bai, dona), phicatuac (azk, b-l, get, izt), phicatuic (g-z, izu), phicatuik (urk), phicaturic (amo, osp, mau), phicaturik (larr, urk), phicatuyac (mak), phikatiac (gab, lekb, dom), phikatiak (a-s, bard, d-s, gam), phikatuak (d-l, mil), phikatuik (alo, altz), phikaturic (ath), phikatuyac (bes), phikatzen (lan), picatcen (hir), picatia (ezt), picatiac (anh, arn, ban, beho, bid, ald, ora, d-g, u-g), picatou (aha), picatouäc (hen), picatu (uzt), picatua (bir, ur-p), picatuac (bas, bir, lek, its, ur-p, urr), picaturic (be-l, soh, las, suh), picatuyac (mug), picatzen (iri), pigatouac (luh), pikatiac (bun, kan, iru, mend, ort), pikatiak (aiz, jats, don), pikatouac (sen), pikatu (sar), pikatuac (haz, meh, dono), pikatuak (ahe, bida, zur), pikatuyac (arb, arr), pikatuyak (esp), pikatzen (aiz), pikhatiac (azka, larz), pikhatouac (lah), piquaturic (alt), pphiquatiac (bit)

Pireneo (244): Piréné (ünd, ünd), Pirénéétaco (ain, ain, hen), Pirénétaco (hen), Pirénetaco (d-1), Piréré (altz), Pirene (sarr), Pireneetaco (sarr, hal, hal, jat), Pirene-etaco (arü), Pireneetako (arü), Pirenétaco (beho), Pirenetaco (beho, jat, larz, larz, meh, meh, zur, zur), Pirenetacotan (get), Pyénéétaco (l-b), Pyenetaco (d-s), Pyrénéac (altz, mug), Pyrénéataco (sen), Pyrénêtaco (bai, bai), Pyrénéétaco (zar, zar, ainh, aint, kan, kan, lah, lah, l-z, l-z), Pyrénéétako (bard, bard, ilh), Pyrénéco (bun, izt, ito, ito, l-s, l-s, mar, mend, z-p, z-p), Pyrénéco_tako (jats), Pyrénéeco (g-z), (Pyrénées) (iho), Pyrénées (aiz, aiz, alt, a-zo, beh, bun, esk, esk, lekb, lekb, o-z, o-z, irur), Pyrénées-mendietako (sar, sar), Pyrénéessétaco (ost), Pyrénées_taco (arh, u-h), Pyrénées-taco (luk, luk), Pyrénées-tako (zib, zib), Pyrénéetaco (ainh, aint, b-l, b-l, iru, jut, jut, las, las, mau, mau, mit, ora, ora, dona, suh, suh, ath, ath), Pyrénéetako (gam, gam, garr, lig, mit), Pyrénéetan (irur), Pyrénéko (a-s, a-s, beh), Pyrénétaco (bit, bit, buz, g-z, hau, hau, iba, iba, izt, lak, mend), Pyrénétako (azka, buz, mil, mil), Pyréneac (mug), Pyrénees_ taco (arh), Pyréneetaco (etx, etx, larr), Pyréneetako (ilh), Pyrénetaco (ürr, ost, dono, urr), Pyreeneco (hoz), Pyrenéétaco (dona), Pyrenéétako (l-b), Pyrenées_taco (u-h), Pyrenées-taco (its), Pyrenées-taho (jats), Pyrenéetaco (be-l), Pyrenéetako (lig), Pyrene (hir), Pyreneac (g-zo), Pyreneco (azk, iri, iri, mak, mak), Pyreneeco (hoz), Pyreneeko (urk), Pyrenees (urk), Pyrenees-taco (its), Pyreneetaco (aha, amo, amo, arn, ürr, be-l, soh, soh, ida, ida, iru, izp, izp, laka, larr, osp, osp, urd, urd, pag, pag, d-i, d-i, zal, ure, ure, bil), Pyreneetako (d-s, sen, bil), Pyreneko (arb, arb, arr, arr), Pyrenesetaco (lek), Pyrenesetako (lek, haz, haz), Pyrenesseco (gab, gab), Pyrenetaco (a-m, aha, anh, anh, azk, azka, aih, aih, ban, ban, bid, bid, bir, bir, bes, bes, gar, get, lak, men, müs, müs, ort, ort, izu, izu, d-l, d-g, d-g, hir, dono, u-g, u-g, ur-p, ur-p, urr, uzt, uzt), Pyrenetako (bida, bida, gar, garr, men), Pyrienoetaco (alo), Pyrineoetako (alo), Pyrinioétaco (a-z, $\mathrm{a}-\mathrm{z})$

plainta (11): plaïntak (altz), plaintac (aiz, ainh), plaintak (jut), pleinta (garr), pleintac (z-p), pleintak (d-b, gam), plenten (sarr), plinten (arü, ünd)

plainu (96): plaïgnouac (lekb), plaignouac (aha), plaignuac (get), plañien (esk), plaïniac (kan, dom), plaïnouac (lah, sen), plaïnuac (b-l, meh), plaïnuyac (bas), plañuac (urr), plagniac (a-z, etx, gab, hau, ida, lig, larr, l-s, l-z, mar, mau, ora, pag, zal, irur), plégniac (bun, iba, ito, ost), plagniak (bard, l-b), plégniak (beh), plagniën (g-z), plagnic (etxa), plagnien (alo, ürr, laka, l-a, men, mit, müs, o-z, arro, bil), Plagnien (alt), plagnik (ath), plagnouac (hen), plagnouak (don), plaiñietara (osp), plaigniac (a-zo, g-zo), plaignuac (bid), pléiniac (aint, zar), plainouac (luh), plainuac (izt), plainuik (urk), plainuyac (bes, its, mak), pléygniac (bit), playgnoac (ur-p), pleñiac (iho), pleigniac (beho, eih), 
pleïniac (anh), pleïnouac (dona), pleïnuac (haz), plegniac (arh, azka, ezt, izp, lan, larz, las, luk, mend, izu, d-g, u-h), plegniak (buz, jats, suh), plegnuac (hoz), pleiñiac (u-g), pleiniac (ban, lak, d-i), pleiniak (a-s), pleinuac (lek, iru), pleinuak (leko), pleinuyak (zur), pleynuak (mil)

planada (5): planada (a-s, buz, iba, l-s, dona)

plano (5): plana (a-zo), plano (bit, g-zo, pag), planou (lah)

plantatu (1): plantatu (anh)

plaza (35): plaçan (aha, amo, beh, bard, etxa, iba, izt, jut, lah, lan, las, luh, mak, mau, mend, d-i, don, ure, ur-p), plaçara (ora), plaçaren (ora), plaçat (bard), plasan (ald), plassan (lig, irur), plazaat (bas), plazalat (urk), plazan (bas, bir, lek, esk, gam, get, haz)

plenta (1): plentac (a-m)

pobre (462): paubre (a-s, a-s, mend, mend), paubriago (a-s), paubriak (a-s, mend), paubrio (mend), pobré (bun, bun, bun, kan, kan, hen, iba, iba, lah, lah, lah, z-p, z-p, z-p), pobréäc (hen), pobréago (hen), pobréagoac (z-p), pobréaguac (izt), pobre (a-m, arh, arh, arh, aih, b-l, b-l, ban, bas, bas, beho, bid, bid, bir, bir, bes, bes, bes, buz, buz, eih, ezt, ezt, gam, hen, iru, iru, iru, izp, izp, its, its, jats, jut, jut, lekb, lekb, lekb, las, las, ald, luh, luk, luk, mak, mak, leko, ora, ora, ort, d-g, d-g, d-g, d-i, hir, hir, hir, sar, sar, dono, dona, dona, suh, suh, suh, u-g, u-g, u-h, u-h, u-h, ure, ure, ure, ur-p, ur-p, urp, urr, mil, mil), pobreaüak (leko), pobreac (ahe, b-l, izt, izt, its, luh, ort, d-i, d-i, sen, dono), pobreago (b-l, iru, its, jats, luk, d-i, sar, ure, mil), pobreagoac (bid, luh, hir), pobreak (leko, leko, sar, mil), pobriô (zur), pobriac (, amo, amo, amo, arh, aih, ban, ban, bas, bid, bir, kan, ezt, get, haz, iba, izp, izt, jats, jut, lah, las, ald, ald, luh, mak, ora, ort, dono, dona, dom, dom, u-g, u-h, ur-p, urr, urr), pobriago (amo, arh, beho, bes, buz, eih, kan, ezt, lekb, mak, ort, dono, dona, suh, u-h), pobriagoac (lah, ora, d-g, u-g, urr), pobriagoak (bas), pobriagouac (las), pobriaguac (izp), pobriak (beho, buz, gam, gam, zur, zur, zur), pobrio (a-m, bir, bun, gam, iba, jut), pobrioocoac (aih), praübé (etxa, hau, hau), praübe (aiz, ainh, ainh, ainh, arü, arü, arü, ürr, ürr, sarr, sarr, sarr, d-b, d-b, ünd, ünd, ünd, etxa, etxa, gab, garr, garr, hau, mau, mau, mau, mit, mit, mit, s-g, s-g, s-g), praübeago (mau), praübe_ago (hau), praübiaüak (d-b), praübiac (gab), praübiago (etxa), praübiagouac (s-g, ath), praübiak (d-b, garr), praübigo (sarr, ünd), praübigouak (ürr, mit), praübio (gab), prabiagouac (irur), praoué (altz, altz, bard, l-s, l-s, l-s), Praoué (altz), praoubé (a-zo, beh, g-z, etx, ida, ito, ito, lig, l-z, ost, ost, ost, o-z, o-z, o-z, ath, irur), praoube (alo, a-zo, bar, g-zo, g-zo, etx, etx, ida, ida, laka, laka, lig, lig, l-z, l-z, l-a, l-a, l-a, l-o, l-o, l-o, men, men, men, irur), praoube_ago (l-a), Praoubec (etx, l-z), praoubek (alo), praoubiai (lig), praoubiac (a-zo, bit, bit, bit, g-zo), Praoubiac (bit), praoubiago (g-z, laka), praoubiagoac (g-zo), praoubiagoak (l-b), praoubiagouac (a-zo, etx, ito, l-z, l-o, o-z), praoubiagouak (ilh, don), praoubiagouér (bit), praoubiak (beh, beh, ilh, ilh, ilh, l-b, l-b, l-b, don), praoubien (don), praoubier (irur), praoubigo (men), praoubio (ost), praouiagouac (l-s), praouiagouak (beh), praouiak (bard), praouyac (bard), praouyagouac (bard), praué (altz, altz), praubé (alt, g-z, g-z, don, ath), Praubé (alt), praubé_ago (alt), praube (alo, alo, ürr, bar, bar, be-l, be-l, be-l, soh, soh, soh, esk, esk, esk, gab, garr, laka, lan, lan, mar, müs, müs, müs, urd, urd, arro, zal, zal, irur, bil, bil, bil), praube_ago (be-l, soh, bil), praubiac (lan), praubiago (ainh, alo, arü, ida, müs), praubiagouac (lig), praubiagouak (mar), praubigo (esk), praubigouk (bar), praue (g-i, g-i, g-i, osp, osp, osp), praue_agouac (osp), prauve 
(larr, larr, larr, mar, mar, mar, pag, pag, pag), prauveagouac (larr), prauviago (pag), probe (anh, anh, arm, arm, lek, lek, lek, get, jats, larz, larz, izu, izu, izu, urk), probiac (anh, arm, lek, get, larz, izu), probiago (anh), probiagoac (arm, lek), probiaguac (larz), probik (urk), probio (izu, urk), probre (dom), probriac (lek)

pollit (85): poïlit (aiz), poïllit (zar, izt), poïlllit, aint), poilit (lak), poillit (a-m, amo, arm, bes, d-s, eih, kan, g-zo, ezt, gam, iho, izp, jats, jut, lekb, las, luk, mak, izu, bai, dona, dom, suh), polit (mar, dono), pollit (aha, arn, a-s, azka, beho, b-l, ban, bas, bid, buz, hel, hen, hoz, iri, iru, larz, l-o, mend, ost, ort, hir, u-g, ure), pollitbatian (anh), pouillitgno (a-z), pouillit (ain, bard, etxa, ilh, ito, l-b, lah, l-s, ora, sen), poulibatian (d-g), poullit (beh, gab), puillit (azk, lek, zib, get, haz), pullit (bir, d-b, its, lar, leko, mug, d-i, zur, ur-p, uzt, mil)

porrokatu (1): phorrokatuz (zal)

potxa (1): potcha (lekb)

prestu (5): perestua (lek), Pestua (haz), Pherestia (dom), Pherestu (esk), Prestuya (mug)

presuna (1): presuna (urr)

probatu (1): probatcen (altz)

pundu (15): phündüradino (d-b), phoundian (buz, gam), phoundouradio (bit), phundaraïnokoa (garr), phundian (a-m, amo, hoz, mend), phunduraano (bes), poundourano (bun), pundian (ban, jats), puntin (men)

punta (6): phuntalat (garr), pountan (luh), puntan (bard, beho, u-g), puntarat (zur)

putzu (34): phoutçou (iba, jut), phoutsou (arh), phoutzou (lekb, dona), phutçu (a-m, amo,

l-b, izu, s-g, ure), phutzu (bas, beho, d-s, lan, larz, bai, u-h), phuzu (l-o), poutçou (aha, mend, ora), poutsou (kan, ald), poutsoubat (bun), putçu (azk, meh, meh), putsu (ban), putzu (alo, ezt, iru, ort)

sain (1): Sain (arü)

saindu (1): Saindua (mar)

Saint Bertrand de Cominges (189): saint Pierrekin (bard), saint Pierré (bard), Saint Bertrand de Commingeko (ahe), Saint Bertrand Cominyeco (anh), Saint Bertrand de Comminges (arb), Saint Bertrand de Comminges (arr), Saint Bertrand de Comminges (bar), Saint Bertrand de Comminges (g-z), Saint Bertrand de Commingeko (laka), Saint Bertrand de Comingesco (izu), Saint Bertrand de Comminges (don), Saint Bertrand Commingeco (zal), Saint-Bertrand-de-Comminges co (eih), Saint-Bernard-de-Cominges eko (bil), Sjan Bertran Comminjeco (iba), St Vindent de Commingeko (aiz), St Bd Comminge (alt), St Bertrand de Commingeco (arh), St Bertrand-de-Comminges (beh), St. Bertrandde-Commingeco (ban), St Bertrand de Comminges (bas), St Bertrand-de-Comminges (bid), St Bertrand de Comminges (lek), St Bertrand Gomminge (mar), St Bertrand de Comminges (mau), St Bertrand-de Comminjeco (men), St Bertrand-de-Comminges (oz), Yondoni Beñat Commingeco (gab), Begnade Commingéco (hen), Beltran Cominyeco (ezt), Beltran Comingeco (iru), Bernat Comintseneco (meh), Bertran Commenyeco (azo), Bertran Cominjekoaren (d-s), Bertran Commingeco (g-zo), Bertran Comminjeco (iba), Bertran Comminyeco (iri), Bertran Commeinge (l-b), Bertran Cominge (lan), Bertran Commingaco (osp), Bertran Commingeco (ort), Bertran Comingoaren (d-1), Bertran Comminges-co (ath), BertranCommingeneko (a-s), Bertrand Commingeco (ainh), Bertrand Comminges (ame), Bertrand Comminges (aih), Bertrand Gommingeko* (ald), Bertrand Commingeaco (arro), Bertrand Commingeco (hir), San 
Bertrand de Comingeco (dono), BertrandComingeko (d-g), Bertrand-Commingeco (uzt), Bertrand-de-Commingeco (ban, mak), Bertrand-de-Commingeneko (mil), Bertrand-de-Comminges (esp, garr), Bertrand-de-Commingeseco (s-g), Bertrand-deComminges-eko (arü), Bertran-de-Commingeco (lar), Bertrann-Cominjaco (hau), Don Betan Comminges (lekb), San Betran Cominyeco (hal), jat), San Betran Comingeko (ilh), san Betrande Cominyeco (u-g), Betran-de-Commingues (altz), Dona Bettan Commingueko (bard), Doni Bettan Commingeseco (ost), Saint-Bertrand de Cominges (Saint-Bertrand de Cominges (amo), Cominseco (iho), San Betran Cominyeco (hal, jat), san Betrande Cominyeco (u-g), Ssan-Bértrann Commingéco (bit), SaintBertrand de Commingeco (hel, hoz), Saint-Bertrand-de Commingeco (jut, mit, lig), Saint-Bertrand de Commingeco (dom), San-Bertrand-de-Commingeco (suh), SanBertrand-Commingeko (azka), Saint-Bertrand de Commingen (arm), Saint-Bertrand de Comminges (etxa), Don Betan Comminges (lekb), Saint-Bertrand-de Comminges (sen), Yon Doni Bettan Commingeseco (ost), Dona Bettan Commingueko (bard), San-Bertrand Comminjaco (izt), Joundane-Betran-Commingeco (l-z), Saint-Bertrandde-Comingeko (buz), Saint-Bertrand-de-Cominges-co (zib), Saint-Bertrand-deCominjeko (gar), Saint-Bertrand-de-Commingéco (bun, kan), Saint-Bertrand-deCommingeco (bir, izp, leko, dona, irur, u-h, ur-p, z-p), Saint-Bertrand-de-Commingeko (l-o), Saint-Bertrand-de-Comminges (azk, be-l, soh, d-b, haz, lak, l-a, luk, müs, ora, sar), SaintBertrand-de-Comminges (alo), Saint-Bertrand-de-Commingeseco (ain), Saint-Bertrand-de-Comminges-eco (esk), Saint-Bertrand-de-Comminges-eko (sarr, ünd), Saint-Bertrand-de-Comminges-ko (jats), Saint-Bertrand-de-Comminjeko (ürr), Saint-Bertrand-de-de-Comminges (lah), Joundaen-Bertrand-Commigaco (ida), Joundane-Betran-Commingeco (etx), Saint-Bertran-Comminjeco (urd), SaintBertrand-Commingesco (las), Saint-Bertrand-Commingesgo (bida), Sen-BertranCommingeco (g-i), Simbertrand-de-Commingéco (l-s), St-Bertrand-de-Comminges (gam), Yondane-Bertrand-Commingeco (pag), Yondoni-Petan-de-Commingesgo (zur), San-Bertran-Commingesseko (a-z), san-Bertran-Comminyekuaan (bes), San-BertrandComingeko (luh), San-Bertrand-Commingeaco (d-i), San-Bertrand-Commingeco (get), san-Bertrand-Comminges (urr), San-Bertrand-de-Commingeco (b-l, larz, larr), San-Bertrand-de-Commingecouain (mend), San-Bertran-de-Commingeko (ure), San-Bertran-de-Comminges-co (its), San-Bertran-de-Comminye (ito), San-BetranCominyeco (bai), San-Betran-de-Comminyeko (urk), San-Pétan-Commenyéco (zar), san (a-s, esp, l-b, s-g, d-g, d-g, d-g, u-g), San (ainh, amo, amo, a-s, d-s, garr, hal, ilh, iri, jat, lan, lar, osp, mak, meh, ort, ort, ort, d-l, hir, dono, uzt, mil, mil, mil), sànn (hen), Bertrand-de-Cominjeco (arn), Saint-Bertrand-de Comingeco (aha)

Saint Pierra (3): Saint Pierra (bil), Saint Pierra ekin (bil), St Pierraekin (mar)

salbaia (57): çalbaya (ald), chalbaya (sen), jalbaya (ost), salbaïa (buz, iru, lah, mend), salbaïya (bida, get), salbaia (bid, lak, d-g, mil), Salbaia (lek), salbaya (a-m, amo, anh, azk, beho, b-l, bard, bas, bes, eih, kan, g-zo, hen, its, jats, jut, lan, luh, mak, meh, ora, izu, d-l, d-i, don, dono, bai, dona, u-g, urr), salbayé (l-s), salbayaüçu (gab), salbayada (zur, urp), salbaye (gam), salbayia (a-zo, a-s), sjalbayaouçou (iba), sjaldo (iba), ssalbaya (bit), zalbaiada (aiz), zalbaya (u-h)

salbaje (21): sabaje (arh), salbage (alt, etxa, mau, ath), salbajé (hau), salbaje (arü, ürr, altz, be-l, soh, sarr, ünd, g-i, ida, mit, bil, z-p), Salbaje (men), salbajia (mar), salvage (pag) 
salbatu (13): saaltcéa (iba), saaltcea (mend), sabalcéa (ito), sabalsera (lig), sabaltcea (altz, ost), sabaltcera (irur, $z-p$ ), sabaltséa (g-z, o-z), sabaltsèra (bun), sabaltsera (ürr), sabaltzera (l-o)

salbatzaile (11): Gure-Salbazailia (larz), Salbaçalia (men), Salbaçaliac (urd), Salbatçailea (lar), Salbatçailia (azk), Salbatxaïlea (arb, arr), Salbatzailea (arb, arr), Salbatzailiac (lak), Salbazaile (lan)

salbu (1): salbu (lar)

saldo (50): saldo (a-m, aint, beho, zar, ainh, amo, altz, bar, bun, d-s, sarr, ünd, esk, etx, gam, g-i, hau, hoz, ida, izp, laka, lig, larr, osp, l-z, l-a, men, müs, urd, o-z, ort, izu, arro, s-g, d-i, don, zal, dona, ath, irur, bil), saldoat (jats), saldobat (alo, arü, gar, l-s, l-o, mit, pag) samin (1): saminak (ahe)

samur (4): samoretaz (lak), samur (bard, dom), samurrez (dono)

samurtu (2): samurtuez (ure), samurtuik (urk)

San Bernard de Comminges (3): St_Bernard_de_Comminges (beho), Saint-Bertrand-de (aha), Saint-Bertrand-de-Comminges (eih)

San-Petan-Commenges (4): San-Bertrand-de-Commingeseco (a-m), San-Pétan-Commenyéco (aint), San_Pierre (beho), San_Pierrekin (beho)

sarrera (1): sarreran (zib)

sarri (29): Hainsari (lak), sari (ürr, ürr, etx, ida, l-z, men, urd, o-z, ure, bil), sarri (amo, arü, altz, be-l, soh, ezt, etxa, gar, g-i, hau, laka, mit, mit, pag), tsari (sarr, ünd, mau)

sartu (343): çareraci (urd), çar_haraci (hen), çarri (ath), çartcin (mau), chararazi (sen), charraraci (ito), chartzeat (aha), chartcerat (sen), chartouac (sen), chartouik (aha), chartséa (ito), jsara'asi (ban), sar (alo, arh, eih, kan, zib, esk, etxa, lig, l-o, mau, o-z, d-i, dom, irur, u-h, uzt), sar_aaci (leko), sar_éaci (alt, etx), saréaci (ath), saraci (gab, hau, izu), sar_aci (anh, men), sararaci (azk, get), sar_araci (ain, arh, be-l, bida, soh, etxa, iru, lah, ora, dona, urr), saréraci (z-p), sar_arasi (kan, u-h), sararasten (luh), sararazi (arr, d-l), sar-arazi (iho, luk, sar), sararazteco (iri), sar-arazten (iri), sar-eaci (arü, sarr, ünd), sar_hazi (haz), charraaci (aha), saraaci (a-m), sarraaci (aint), sar_arazi (a-s, aih, lak, bai), sarartino (alt), sar-artino (l-o), sarartio (l-s), sarassi (l-s), sarazi (urk), sarcea (hel, men), sarceacoan (ure), sarceat (a-zo, bir), sarcera (alt), sarcerat (azk, d-g), sarciten (lar), sareaci (pag), sar_eaci (bil, l-z, mit), sareraci (mar), sar_eraci (amo, b-l, g-z, g-i, ida, larr, osp, l-a, mak, mau, müs, arro, s-g, zal), sar_erazi (bar, esk, gar, lekb, dom), sar_errassi (ürr), sarhaaci (beh), sar_harasten (ald), sarharazi (zib), sarraaci (aint, zar, las), sarraasten (a-zo), sarraatci (mend), sarraazi (azka, bas, bes, d-b, gam, hel, jut, larz), sarraazten (g-zo), sarraci (ilh, l-b), sarraraçi (ort), sarraraci (a-z, beho, lek, esp, hoz, its, mug, d-i, hir, suh, ure), sarréraci (ainh), sarrarasi (izt), sarrarazi (arb, bid, buz, ezt, hal, izp, jats, meh, dono, u-g, mil), sarraraztén (don), sarrarazten (ahe), sarrartio (jats), sarrasteco (altz), sarrasten (lan), sarrâzi (zur), sarreci (bard, bard, bir), sarreraci (laka), sarrhassi (bun), sarsiakin (bard), sart (bas), sartçera (luk), sartcéa (a-z, hau), sartcéra (ainh, don), sartcérat (ain, hen), sartcea (etx, ilh, jut, l-b, l-z, leko, mend, o-z), sartceat (bida, g-zo, gab), sartcera (amo, anh, arh, arm, arü, be-l, bid, soh, sarr, ünd, esp, etxa, g-i, hal, ida, jat, laka, larr, las, osp, mak, mar, mau, mug, müs, ora, izu, pag, arro, s-g, suh, ath, irur, ure, bil, z-p), sartcerat (arn, b-l, eih, get, hoz, iri, izt, its, lan, ald, meh, ort, d-i, u-g, urr, uzt), sartciarekin (mar), sartciten (hoz), sartera (altz), sarthiac (buz), sarthou (aint, eih, zar, larz, ald, ald, leko, ora), sarthu 
(arn, a-z, azk, bida, d-s, hoz, iri, izp, its, laka, luk, hir, sar), sarthuac (hel), sarthuak (d-l, mil), sarthuric (g-i, ida, las), sarthurik (ürr), sarthuyac (mak), sartiac (bid), sartou (hen, don), sartséa (kan), sartséra (l-s), sartsera (g-z, lig), sartu (lek, lek, esp, haz, o-z, d-g), sarturik (mit), sartzéra (lah), sartze (urk), sartzea (bes, buz, d-b, gam, jats, larz), sartzeat (bas, iho, dono), sartzen (ahe), sartzera (alo, ürr, bar, esk, gar, izp, lekb, l-a, mit, d-l, sar, bai, dona, dom, u-h, mil), sartzerat (a-m, arb, arr, a-s, azka, beho, d-s, ezt, haz, lak, l-o, zur), sartzian (iru), sartziarekin (luk), sartziten (iho), sharcèa (bun), ssar_araçi (bit), ssartcéra (bit), ssarthou (bit), zar (ame, a-s), zar_arazi (ame), zarcen (ur-p), zarrarazi (d-s, jat), zarrazi (garr), zartceat (beh), zartzera (ame)

segatu (7): segatiac (ure), segatoua (hen), segatuac (meh, dono, urr), segatuya (bes), segatuyak (esp)

segitu (53): chéguitiac (aha), jseguitiac (ban), séguitiac (a-z, bit, bun), séguitouac (hen, lah), séguitouyac (kan), séguituyac (mug), séitiak (ilh), segitia (luk), segitiac (beho, u-g, u-h), segitiak (buz, d-b, d-g), segituac (hoz), segituak (haz), segituyac (bes), seguitiac (a-m, anh, arh, arm, eih, gab, iru, jut, lekb, ald, mend, ort, bai, dom, suh), seguitiak (bard, jats), seguituac (azk, b-l, lek, izt, dono), seguituak (bida, esp, mil), seguituric (amo, arü, las), seguituyac (mak), seigitiak (l-b), sjeguitiac (iba), zeguituak (aiz)

segur (40): ségur (g-z, o-z, z-p), ségurréna (don), séhur (beh), seür (d-b, las), Se’ur (ban), segour (a-s, eih, luh), segur (a-zo, beho, ürr, bar, esk, ezt, etxa, hoz, laka, lan, larr, osp, mau, mit, mug, müs, urd, urd, pag, arro, d-i), seguric (sen), segurik (bida), segurtameneki (g-i), seour (g-zo), seur (iho), seurraz (d-b), sségourki (bit), zegur (bar) segurki (102): cheourki (aha), jesurki (ost), ségourki (kan, lah), ségourqui (hen), ségurki (az), séourki (zar, azka), séurqui (l-s), seürki (gab), seürqui (bai), seûrki (zur), se’urki (ban), segourki (arm, buz, lekb, ald, ora, dona), segurki (alo, anh, arb, arn, arr, arü, azk, altz, aih, bas, be-l, soh, sarr, esp, etx, gar, get, haz, hau, hoz, ida, iri, iru, izt, its, l-b, lak, laka, lig, larz, l-z, l-a, l-o, luk, mak, mar, meh, men, izu, s-g, d-g, sar, zal, dono, dom, suh, ath, irur, u-g, ure, urr, uzt, uzt, mil, bil), segurqui (amo, hir), segurtki (ame), séourki (aint), seourki (arh, gam, hel, jut, mend, ort), seurki (a-m, a-zo, bard, bid, bes, d-s, g-zo, iho, ilh, izp, jats, urk), seurqui (bir), sjéourki (iba), zeürki (aiz), zegurki (ünd, u-h)

sekula (1): seculan (mug)

seme (145): chémér (ito), chemer (aha), jémér (ost), jsemer (ban), sémééri (ain, hen), sémér (bun, kan), sémer (aint, bit, g-z, hau, l-s, ath, irur, z-p), sémeri (don), sémerri (lah), semeï (eih, lar, sen), semeari (azk, b-l), semeer (iru), semeeri (ahe, zib, iri, luh, sar), semei (hal, its, jat), semer (a-m, arn, beho, zar, ainh, alt, alo, amo, anh, a-z, a-zo, arh, arm, arü, ürr, a-s, azka, altz, aih, beh, bard, be-l, bid, g-zo, soh, sarr, d-b, ünd, esk, ezt, etx, etxa, gab, gar, g-i, hel, hoz, ida, iho, ilh, izp, izt, jats, jut, l-b, lak, laka, lekb, lig, lan, larz, larr, las, osp, l-z, l-a, l-o, luk, mar, mau, leko, mend, mit, müs, ora, urd, o-z, ort, izu, pag, arro, s-g, zal, dono, bai, dona, dom, suh, u-g, u-h, ure, bil), semeri (bida, bir, esp, haz, ald, mak, meh, d-l, hir, urk, mil), semerri (bes, mug), semier (bar, lek, d-s, buz, gam, d-g, zur), semieri (arb, arr, ur-p, urr, uzt), sjémer (iba), zemer (aiz, ame, garr), zemeri (get)

senar (160): cennharra (hen), chenarra (aha), chenhar (ito), chenharra (ito, sen), jenharra (ost), sénhara (kan, l-s), sënharra (g-z), sénharra (ain, don), sénharrac (bun), senarha (meh), senarra (bid, bir, esp, haz, jats, ald, zur, ur-p, urr), senarrac (aih), senarrak 
(zib), senhar (ame, ame, altz, altz, altz, altz, bas, bida, iho, lar, lar, lar, müs, d-g, sar), senhara (alo, anh, arü, azk, etx, hau, ida, lak, lekb, lig, l-z, l-a, l-o, mar, mau, müs, urd, o-z, pag, d-i, sar, dom, ath, irur, bil, z-p), senharra (a-m, aint, arn, beho, eih, zar, ainh, alt, ame, amo, a-z, arb, a-zo, arr, arm, ürr, a-s, azka, beh, b-l, ban, bar, bard, bas, be-l, bida, lek, bes, d-s, buz, g-zo, soh, sarr, d-b, ünd, esk, ezt, etxa, gab, gam, gar, g-i, get, hel, hal, hoz, iho, ilh, iri, iru, izp, izt, its, jat, jut, l-b, laka, lan, larz, larr, lar, las, osp, luk, mak, leko, men, mend, mit, mug, ora, ort, izu, arro, s-g, d-g, zal, dono, bai, dona, suh, u-g, ure, uzt, mil), sjenharra (iba), zenhar (arh, garr, garr, u-h), zenharra (aiz, arh, $\mathrm{u}-\mathrm{h}$ ), Zenharrak (garr)

senar-emazte (4): senaremazte (zib, zib), senhar-emazte (iho, d-l)

senar-emazteki (1): senhar-emaztekia (d-l)

sentitu (1): sendi (altz)

sinetsi (162): chinésdéçacié (ito), chinétchi (ito), chinetch (aha), chinetch-çaçoue (sen), jinhetx (ost), sinnhex (g-z), sïnhexi (g-z), Siñets (zib), sihesten (lek), sinéts (don), sinétsazié (azka), sinets (hen, its), sinetsacie (arm), sinetsazii (bes), sinetsazou (gam), sinetsazu (zur), sinexi (beh), sinhési (alt), sinhétsi (l-s), sinhest (iru), Sinhestén (l-s), sinhesten (o-z, hir, dom, bil), sinhets (ain, arn, beho, zar, alo, bas, d-s, buz, kan, esp, esk, etx, etxa, gab, hel, hal, haz, ida, iri, izp, izt, jat, jats, jut, l-b, lekb, lig, larr, las, l-z, mau, meh, men, ora, o-z, s-g, d-l, d-i, sar, zal, suh, irur, u-g, u-h), Sinhets (luh), sinhetsaçoue (ort), sinhetsacié (aint, ilh), sinhetsacie (leko), sinhetsassou (bun), sinhets-assuë (luk), sinhetsazîe (bai), sinhetsazu (urk), sinhetsazue (bid), sinhetsi (a-z, altz, bar, bard, d-s, d-b, esk, etx, ilh, l-b, laka, lah, l-z, men, urd, ort, d-i, urk), sinhetss (ald), sinhetx (a$\mathrm{m}$, arb, arr, arü, sarr, mak, dona, urr), sinhetxaçue (b-l), sinhetxezazie (ünd), sinhetxi (ezt), sinhetz (ame, a-s, azk, bard, dono, ath), sinhetzçacie (d-g), sinhetzi (alo, ame, ath), sinhex (ainh, amo, anh, a-z, ürr, be-l, soh, gar, g-i, hau, lah, mar, mit, mug, müs, izu, pag, z-p), sinhexa (eih), sinhexaçu (beh, lar), sinhexaçue (ure), sinhexazie (larz), sinhexazu (lak), sinhexi (a-zo, arü, be-l, g-zo, soh, gar, g-i, lig, osp, arro), sinhez (mil), sinhezcasié (l-o), sjinetjacie (iba), Ssinhestènn (bit), zinets (get), zinhetz (aiz), zinhetzi (garr)

so egin (1): soguin (alt)

soegile (1): so eileric (izp)

sokorritu (114): chocorritsén (ito), jokhorritcén (ost), chokhorritzen (aha), sécouritsen (1s), sécouritu (l-s), sjokhorritcén (iba), socchorritu (a-zo), Soccorri (alt), soccorritcen (its), socoritcen (mau, d-i), socoritzen (bid), socorricen (bir, ur-p), socorritcen (ainh, get, dona, suh, u-g, uzt), socorritsen (ald), socorritu (ainh, beho), socorritzen (beho), sokhoritcen (etx, etxa, hau, lig, l-z, müs, izu, irur, urk, z-p), sokhoritsén (g-z), sokhoritu (a-m, etx, gar, l-z, l-a, mak, müs, dom, z-p), sokhoritzen (a-s, b-l, mit), sokhorri (urk), sokhorritcen (a-z, arü, altz, bard, be-l, soh, sarr, ünd, g-i, ida, ilh, l-b, laka, larr, mug, ora, s-g, d-g, ath, ure, bil), sokhorritou (a-s), Sokhorritou (don), sokhorritsen (u-h), sokhorritu (ahe, a-z, arb, arr, azka, altz, b-l, g-zo, g-i, luk, d-g, zal, u-g), Sokhorritu (larz), sokhorritzen (alo, ürr, azka, bar, d-b, esk, gam, iho, iru, lan, larz, l-a), sokoritcen (mak, mar, men, dono), sokorritcen (amo, mend), sokorritu (arm), sokorritzen (ahe, jats, lekb, zur), ssokhorritou (bit), zokhorritzen (arh), zokorritcen (beh), zokorritzen (ame)

soleitu (4): soleatzen (izp), soleitcen (a-m), soleitu (bas, bes) 
soro (51): chorroac (sen), sohouac (alt, be-l, g-z, etx, g-i, hau, laka, l-z, l-a, müs, urd, o-z, zal, ath, z-p), sohouak (alo), sohouc (soh, esk, bil), sohouen (altz), sohouk (bar, men), sorhoac (ain, arb, arr, hir, urr), sorhoak (sar), sorhouac (ainh, ida, lig, larr, osp, mau, s-g, irur), sorhouäc (hen), sorhouak (ürr, gar, mit), sorhouc (arro), sorhuac (azk, bir, get), sorhuak (bida, d-l), sorhueri (zib), sorhuk (urk), sorruac (ur-p), sorruak (zur)

soropil (1): sohapilla (beh)

sostengatu (1): chochtengatou (aha)

su (35): su (arn, eih), sou (arh, bun, lekb, lah, ald, ora, don), su (ain, ahe, ahe, anh, arb, arr, ban, zib, haz, iri, iri, ito, its, jat, lar, lar, l-a, l-o, mak, mend, izu, arro, d-i, u-h, urk, uzt) sukalde (5): çoucalde (hen), shukhaldian (l-s), sukaldian (urr), sukhaldean (hir), sukhaltian (zal)

suntsitu (14): çouncitou (hen), chounchitou (sen), sounsitou (lah), sountsitou (ain), sunsitu (azk), suntcitcen (esp), suntsitcen (uzt), suntsitu (bas, zib, hal, its, jat, meh, zur)

supazter (96): jou bajterriane (ost), chou bazterrean (sen), chubasterrian (d-g), soubazterrian (don), sou pazterrian(ora), soupazterrian (arm, jut), souphastérian (kan), souphasterrian (hel, zar), souphazterrian (aint), subasterrian (azka, lek, u-g), subazterrean (sar), su basterian(ban), mak), su basterrean(uzt), su basterrian(jat, u-h), su bazterian(lekb), su bazterrean(haz), su bazterrian (aih, arh, buz, ezt, hal, l-b, las, luk, meh, s-g, bai, dona), su pastarrian(ito), supaster (alt), su pasterean(zib), supasterian (mar), su pasterrean(its), supasterrean (leko, mug, mug, ure, mil), supasterria (ur-p), su pasterrian(arb, arr, izu, lah, a-z, a-zo, bas, bir, d-b, esp, mend, dono, suh), supasterrien (bard), su pazterian(anh), supazterrean (ort, d-l), supazterrian (bid, gab, gam, iru, izp, jats, lak, lan, zur), suphasterian (azk), suphasterrean (b-l, b-l), su phazterin(urk), su phasterrean(d-i), su phasterrian(lar, lar), su phazterrean(iri), suphasterrian (amo, beho, d-s, g-zo, get, hoz, ilh), suphasterrin (bes), suphazterian (a-m), suphazterréan (izt), suphazterretan (iho), suphazterrian (iho, larz, dom), zupasterrean (ame), su pphazterrin (arro)

supizgia (1): souphizguian (a-s)

suspira (1): souspirac (hel)

sutondo (48): chout ondoan (aha), sou én oundouan(bun), sou ondoan(ald), su ondoan(ahe, ahe, ain, don, iri), su ondouan(l-a, l-o), southonduan (iba), suthondo (bil), suthondouan (alo, be-l, soh, etx, g-i, ida, lig, osp, l-z, mau, mit, müs, urd, o-z, pag, ath, irur, z-p), (suthondouan) (urd), suthondouann (hau), suthondoun (arü, ürr, altz, bar, g-z, sarr, ünd, esk, laka), suthondoutan (bar), suthondun (ainh), suthoundoun (etxa), suthundouan (larr), sutondouan (gar), sutondoun (men), sututsun (mit)

tailatu (4): thaillatia (beho), thaillatuic (hau), thaillaturic (larr, s-g)

Tantugu (781): Tangugou (sarr), Tanguguri (hir), Tantügou (d-l, urk, urk), Tantügoui (urk), Tantügu (d-l, d-l, d-l), Tantüguri (d-l), Tantaguri (u-g), Tantiguren (get), Tantougou (arh, arh, arh, arh, bit, bit, bit, bit, bun, buz, buz, buz, buz, g-z, garr, garr, jut, don), Tantougouén (l-s), Tantougouènn (bit), Tantougouen (l-a, don), Tantougouin (arm), Tantougouren (buz), Tantougouri (bit, garr, don), Tantugü (d-b, d-b, d-b), Tantugük (d-b), Tantugüri (d-b), Tantugic (get), Tantugo (esk, luh, luh), Tantugoûn (bai), Tantugoin (l-b), Tantugoren (ame), Tantugori (luh), Tantugou (a-m, aiz, aiz, aiz, aiz, ain, ain, ain, ain, ain, ain, a-m, aha, aint, arn, beho, eih, zar, zar, zar, zar, ahe, ahe, ahe, ahe, ahe, ahe, ahe, alt, alt, alt, alt, alo, alo, alo, alo, ame, ame, ame, ame, ame, ame, , 
amo, amo, amo, amo, anh, a-z, a-z, a-z, arb, arb, arb, arb, a-zo, a-zo, a-zo, arr, arr, arr, arr, arm, arm, arm, arm, arü, arü, arü, arü, arü, ürr, ürr, ürr, ürr, ürr, a-s, a-s, a-s, azk, azk, azk, azk, azk, azka, azka, azka, azka, altz, aih, aih, aih, aih, aih, beh, b-l, b-l, b-l, b-l, b-l, b-l, ban, ban, ban, ban, bar, bar, bar, bar, bar, bard, bard, bard, bas, bas, bas, bas, bas, bas, be-l, be-l, be-l, be-l, be-l, bid, bid, bid, bid, bida, bida, bida, bida, bir, bir, bir, bir, lek, lek, lek, lek, lek, bun, bun, bun, buz, kan, kan, kan, kan, kan, g-z, g-z, g-z, g-zo, g-zo, g-zo, soh, soh, soh, soh, soh, sarr, sarr, sarr, sarr, zib, zib, zib, ünd, ünd, ünd, ünd, ünd, esp, esp, esp, esp, esp, esk, esk, esk, esk, ezt, etx, etx, etx, etx, etx, etxa, etxa, etxa, etxa, etxa, gab, gab, gam, gam, gam, gam, gam, gar, gar, gar, gar, gar, garr, g-i, g-i, g-i, g-i, g-i, hel, hel, hel, hel, hal, haz, haz, haz, hau, hau, hau, hau, hau, hen, hen, hen, hen, hen, hoz, hoz, hoz, iba, iba, iba, ida, ida, ida, ida, ida, iho, iho, iho, ilh, ilh, ilh, iri, iri, iri, izp, izp, izp, izp, izt, izt, izt, ito, ito, ito, ito, ito, ito, ito, its, its, its, its, jat, jats, jats, jats, jats, jut, jut, jut, jut, l-b, l-b, l-b, lak, lak, lak, lak, lekb, lekb, lekb, lekb, lekb, lig, lig, lig, lig, lig, lig, lah, lah, lah, lan, lan, lan, larz, larz, larz, larr, larr, larr, larr, lar, l-s, l-s, l-s, las, las, las, las, ald, ald, ald, ald, ald, ald, osp, osp, osp, osp, osp, l-z, l-z, l-z, l-z, l-z, l-a, l-a, l-a, l-a, l-o, l-o, l-o, l-o, l-o, luh, luk, luk, luk, luk, mak, mak, mak, mak, mak, mar, mar, mar, mar, mar, mau, mau, mau, mau, mau, meh, meh, meh, meh, leko, leko, leko, leko, mend, mend, mend, mend, mug, mug, mug, müs, müs, müs, müs, müs, ora, ora, urd, urd, urd, urd, urd, ost, ost, ost, ost, ort, izu, izu, pag, pag, pag, pag, pag, arro, arro, arro, arro, s-g, s-g, s-g, s-g, s-g, d-i, d-i, d-i, d-i, sen, sen, sen, don, don, hir, sar, sar, sar, sar, dono, dono, dono, dono, bai, bai, bai, bai, dona, dona, dona, dona, dom, dom, dom, suh, suh, suh, suh, ath, ath, ath, ath, ath, irur, irur, irur, irur, irur, u-h, u-h, u-h, u-h, urk, urk, ure, ure, ure, ure, ure, ur-p, ur-p, ur-p, ur-p, ur-p, urr, urr, urr, urr, urr, mil, mil, mil, bil, bil, bil, bil, bil, bil, z-p, z-p, z-p), (Tantugou) (arü), Tantugou (arr, bas), Tantugoui (l-s), Tantugouine (ost), Tantugouc (aha, alt, amo, arn, a-z, a-zo, arm, bid, bir, lek, bun, bun, kan, g-zo, haz, hoz, iba, iho, iri, izt, its, lak, lak, lah, lan, larz, l-s, las, luk, meh, mend, ora, izu, d-i, sen, sen, dono, bai, dom, z-p), Tantugouen (alt, a-z, be-l, soh, etx, g-i, hau, lig, osp, l-z, l-o, leko, müs, pag, irur, u-h), Tantugou-en (bar), Tantugoui (g-z, g-zo, sar, z-p), Tantugouin (azka, bid, hoz, iba, ilh, larz, mend), Tantugouk (ahe, alo, a-s, bard, bida, zib, esp, ilh, leko, mug, don, sar, mil), Tantugoun (bard), Tantugouren (arh, beho, zib, iri, izt, jut, luh, urd, sar, dona, suh), Tantugou-ren (its, sar), Tantugouri (a-m, aint, arn, eih, zar, a-z, arb, a-zo, arr, arh, a-s, azka, ban, bard, lek, hel, haz, hoz, iba, iho, ilh, izp, izt, ito, l-b, lah, lan, larz, larr, l-a, ora, ora, ost, arro, dona, dom, suh, u-h, mil), Tantugou-ri (iri), Tantugouy (irur), Tantugu (anh, anh, anh, anh, arm, arn, bes, bes, d-s, d-s, d-s, d-s, ezt, ezt, gab, gab, get, get, get, hal, hal, hal, iru, iru, iru, iru, iru, jat, jat, jat, jats, lar, lar, lar, ort, ort, izu, hir, hir, zur, zur, zur, u-g, u-g, u-g, uzt, uzt, uzt, uzt), Tantuguc (anh, bes, bes, ezt, hal, jat, hir, u-g), Tantugucen (ort), Tantuguco (get), Tantuguen (ure), Tantugui (zur), Tantuguin (zur), Tantuguk (d-s, zur), Tantugunen (ort), Tantuguren (bes, ezt, hal, iru, jat, lar, u-g), Tantuguri (bes, d-s, ezt, gab, get, lar, ort, izu, uzt), Tantuguyn (d-s), Tartaüa (mit, mit, mit, mit, mit), Tartaüaren (mit), Tartaoua (men), Tartaouaen (men), Tartaouk (men), Tartaroua (ainh, ainh, ainh, ainh, ainh, men, men, men, zal, zal, zal, zal, zal), Tartas (laka, laka, laka, laka, laka), Tartazen (laka), Tentagu (d-g, d-g, d-g), Tentaguk (d-g), Tentaguri (d-g), Tentugou (altz, altz, altz, altz, altz), Tentugouen (altz) 
tapatu (9): tapaturic (be-l, zal), thapatia (l-o), thapaturic (soh, ida, lig, müs, arro), thapaturik (gar)

Tartaroa (22): Tartaüa (mit, mit, mit, mit, mit), Tartaüaren (mit), Tartaorua (men), Tartaouaen (men), Tartaouk (men), Tartaroua (ainh, ainh, ainh, ainh, ainh, men, men, men, zal, zal, zal, zal, zal)

Tartas: (6): Tartas (laka, laka, laka, laka, laka), Tartazen (laka)

tarte (2): tartia (ur-p), tartian (bir)

tiki (25): tiki (azk, azk, azk, bida, bida), ttiki (ahe, zib, zib, d-l, sen, sen, sen, sar, sar), ttiqui (bir, bir, bir, get, get, get, hen, hen, hen, ur-p, ur-p)

tipi (221): thipi (arm, arm, arm, l-z), thipia (bit), thipibat (alt), thipibatec (alt), tipi (aih, bas, bid, kan, gam, gam, gam, hel, hel, jut, jut, jut, lak, lak, lig, lig, luh, luh, irur), tipibat (l-o, mar), tipibatek (l-o), tippi (its, its, its), ttipi (aiz, aiz, aiz, ain, ain, ain, a-m, aha, aint, arn, beho, eih, zar, zar, zar, ame, ame, ame, amo, amo, anh, anh, anh, a-z, arb, arb, arr, arr, arh, arh, arh, a-s, a-s, azka, aih, beh, beh, b-l, b-l, ban, ban, ban, bard, bas, bid, lek, lek, bes, bes, bes, bun, bun, bun, buz, soh, esk, ezt, ezt, garr, hal, hoz, hoz, hoz, iba, iba, iba, iho, iho, iho, iru, iru, iru, izp, izp, izp, izt, izt, jat, jats, jats, jats, lak, laka, laka, laka, lekb, lekb, lekb, lah, lah, lah, lan, lan, larz, larz, las, las, las, ald, ald, ald, luk, luk, mak, mak, mak, meh, meh, meh, leko, leko, leko, mend, mug, mug, ost, ost, ort, ort, izu, izu, izu, arro, d-i, d-i, don, don, don, hir, hir, hir, zal, zur, zur, dono, dono, dono, bai, bai, bai, dona, dona, dona, dom, dom, suh, suh, suh, u-g, u-h, u-h, u-h, urk, urk, ure, ure, ure, ur-p, mil, mil, bil, bil), ttipibat (aha, eih, a-s, esk, arro, mil), ttipibatec (amo, azka, azka), ttipibatek (u-g), ttippi (esp, esp, ort), ttipy (mug)

to (2): Beaouc to (Zar), to (aint)

toki (39): toki (ahe, arh, arn, azk, bas, bes, kan, gab, hel, lah, l-o, meh, mug, ort, d-l, sen, zur,

u-h, mil), Toki (sen), tokian (anh, azk, kan, hel, hal, jat, meh, ort, zur, mil), tokietako (ahe, ahe), tokietan (ur-p), toqui (get, hen, d-i, urr), toquian (hir), toquiann (hen)

tradukzio (1): tradouczionia (buz)

trikatu (1): trikatu (zib)

tropa (52): tropa (ame, anh, arn, a-s, azka, aih, be-l, bit, buz, g-z, soh, ezt, etxa, gab, get, hen, iru, izt, ito, its, jut, lekb, lah, lan, las, luh, mau, meh, ost, d-g, zur, dono, suh, u-g, urp), tropa'at (ban), tropaat (aha, beh, bes, g-zo, mend), tropabat (alt, a-zo, b-l, mil, z-p), tropat (bard, bir, garr, ilh, l-b, urk)

trukatu (2): trucatu (lig, irur)

ttipiño (5): ttipiño (iri), ttipiñoatek (d-b), ttipigno (ezt, larz), ttipi gnobat (mend)

ttipitto (2): ttipitto (uzt), ttipittobat (mug)

ttotto (2): ttoto_eraci (1-a), ttottoaci (ath)

tulumio (1): touloumio (hel)

tulunbio (2): tulumbi (lek), tulunbio (bes)

txakur (11): tçaqur (alt), tchachur (mau), tchacur (etx, zal), tchacurac (l-a), tchakhur (alo, ida), tchakur (gar, hau, osp, l-z)

txipi (86): chipi (a-zo, arü, ürr, ürr, altz, altz, be-l, soh, sarr, sarr, ünd, ünd, etxa, etxa, ito, ito, ito, l-s, l-s, mit, mit, pag), chipibat (arü, mit, z-p), chipibatec (pag, pag, z-p, z-p), chipibatetan (altz), chippi (men, men, men), tchipi (ainh, ainh, ainh, alo, a-zo, a-zo, bar, be-l, be-l, bit, g-z, g-z, g-zo, g-zo, soh, etx, etx, gar, gar, g-i, g-i, g-i, hau, hau, hau, ida, ida, ida, larr, larr, larr, l-z, l-a, mau, mau, mau, müs, müs, müs, müs, o-z, o-z, o-z, 
s-g, zal, ath, ath), tchipibat (alo, ida), tchipibatek (d-g, d-g), tchipy (s-g, s-g)

txipiño (3): etchéchipignoat (a-zo), chipiño (d-b), chipignoat (g-zo)

txoko (2): chokhoun (bil), tchocouan (alt)

tzar (6): (tcarrak) (bida), tzar (zib), tzarrak (zib, d-1, zur), tzarrez (zur)

ukan (161): nahuken (mar), ükhan (d-b, d-b), ükhen (g-z), nahiukhen (laka), nahukhen (ünd, ath), ouhkan (arm), ouhken (bun), oukan (aha), oukhan (aint, aiz, zar, ame, a-zo, arh, arm, arm, a-s, a-s, buz, buz, buz, g-zo, gam, gam, gam, garr, garr, hel, hel, iba, iba, iba, ilh, ilh, ilh, ilh, ito, jut, jut, l-b, l-b, l-b, l-b, l-b, don, dona, dona, dona), oukhançoutènn (bit), oukhane (ost, ost, ost), oukhann (bit), oukhanzouten (a-s), oukhen (bun, bun, lekb, ora), ukan (lek, jats, jats, meh, meh, mend, mend, ort, ort, dono, dono), uken (sarr, mar, mar, bil), ukhan (a-m, ame, arn, a-z, a-z, a-z, azka, beh, beh, be-l, bes, d-s, d-s, d-b, ezt, gab, gab, gab, hoz, iho, iho, iho, iru, iru, izp, izp, izp, jats, lan, lan, larz, larz, luk, luk, luk, luk, mend, izu, izu, izu, don, u-h, urk, urk), ukhën (g-z), ukhén (hau), ukhanez (urk), ukhen (ainh, alo, arü, beho, ürr, soh, etx, etxa, gar, g-i, g-i, ida, laka, lig, lan, osp, l-z, l-a, l-a, l-a, mar, men, men, mit, müs, müs, urd, o-z, o-z, arro, s-g, irur)

ulain (6): ülhañez (d-b), oulhaïn (bun), ulhagnetçaz (mau), ulhagnez (lig, irur), Ulhain (ainh) ume (1): ume (ame)

unai (3): ounhaiez (zar), ounhaïez (aint), ounhayez (eih)

ur (65):-ura (bir), hour (a-s, altz, altz, bar, eih, g-i, hau, lig, mar, don, irur, bil), hourés (z-p), hur (ame, ame, anh, beh, b-l, bid, bes, hoz, urk), our (aint, aiz, zar, a-zo, g-zo, garr, garr, hen, ilh, lah, luh, luh, ora), our (hel), ur (ahe, a-z, aih, bida, bir, lek, esp, gab, haz, lak, lar, luk, mak, mug, hir, sar, zur, dono, dom, suh, ur-p, urr), oura (aint), ur (arn), Ur (ahe, aih), ura (ur-p, ur-p)

urgatzi (2): urgaïzten (gar), urgaizten (zal)

ur-hil (1): ouril (sen)

uri (1): urien (irur)

urlo (1): ourlo (ain)

uros (39): hourous (hen), ouros (aiz, arm, azka, buz, eih, hel, dona), ourosssak (gam), ourouch (aha, sen), ourous (kan, lekb), ourousac (ald), urojsac (ban), uros (ahe, beho, b-l, d-s, ezt, hoz, iho, iru, jats, lak, mend, ort, d-i, bai, suh, u-g), urosac (izp, las), urous (ito, ora), uroz (anh, d-g, urr), ourus (izt)

urratu (3): ourrastéko (lah), ourratceco (arm), urarto (urk)

urrun (154): ürhünetik (d-b), hourounétic (kan), hourroundic (ain, hen), hourrounic (aha), hurüntic (g-z), hurrun (arü, d-l, d-l), Hurrun (g-i), hurrundanic (amo), hurrundic (bid), hurrundik (zib), hurrunetic (s-g, d-g), hurrunti (arü, altz, sarr, larr, urd, pag), hurruntic (ainh, alt, ürr, be-l, soh, ünd, esk, g-i, hau, osp, mit, o-z, arro, zal, dom, ath), hurruntik (bar, gar, laka, men), hurun (zib), hurundic (etx, d-i), hurunic (lak), hurunti (l-a), huruntic (etxa, ida, lig, l-s, l-z, mau, müs, irur, bil, z-p), huruntik (alo, l-o), ourhounic (bit, ora, ost), ourhountic (arh), ouroundik (luh), ourroun (ald), ourrounéti (zar), ourrounétic (bun), ourrounétik (don), ourroundanic (izt), ourroundic (hel, lah, sen), ourrounetic (aint, arm), ourrounetik (a-s, buz), ourrounic (eih, iba, jut, izu, dona), ourrounik (gam), ourrountic (lekb), urhenetik (bard), urhun (beh), urhunétic (ito), urhundic (g-zo), urhundik (bida), urhunetic (luk), urhunetik (garr, ilh), urhunic (a-zo, iho), urhunt (gab), urrhun (l-b), urrhundik (zur), urrun (iri, uzt), urrunétic (a- 
z), urrundaüde (lar), urrundanic (aih, lek, bes, hal, iru, jat, lar, mar, ure), urrundi (urp), urrundic (a-m, arb, arr, b-l, bas, bir, ezt, get, haz, iri, its, mak, mug, hir, bai, u-h, urr, uzt), urrundik (ahe, d-s, esp, sar), urrunetic (aiz, ame, arn, beho, lan, las, mend, ort, u-g), urrunetik (azka, leko, mil), urrunianic (dono), urrunic (ban, hoz, izp, larz, suh), urrunik (jats), urundic (azk, meh), urunetic (anh), urunetik (urk)

urrundu (14): hurruntcen (be-l, soh), huruntzen (l-o), ourhountcén (don), urhunduak (bida), urhuntcen (a-zo, bida, g-zo), Urrundiak (aiz), urrunduric (a-z), urruntaraziac (izp), urruntcen (b-l, its), urruntzen (bas)

urrupea (1): urruphea (etxa)

urrutxuna (7): urruchouna (g-i, laka), uruchouna (ida, urd, irur, z-p), urutchouna (lig)

uste (1): ouste (1-a)

usu (58): üsü (d-b), üssu (bard), ousou (arh), oussou (a-s, bun, mend, ora, ost, ost), uchu (ito), ussu (anh, a-zo, arü, bard, g-zo, etxa, g-i, ilh, l-b, larr, l-s, osp, l-o, mar, mau, müs, o-z, pag, arro), usu (alo, a-m, amo, ürr, altz, bar, be-l, lek, soh, sarr, ünd, esk, etx, gar, hau, laka, l-z, l-a, luk, men, mit, izu, s-g, dono, urk, mil, bil, z-p)

utzi (133): eitci (osp), houtsi (hen), itzitzien (garr), outci (eih, arm, jut, ora, ost, sen, dona), outsi (arh, bit, bun, kan, ald), outzi (aha, ain, a-s, buz, gam, hel, lah), ouztén (don), usten (d-g), utçi (ort), utci (ainh, a-m, amo, anh, arn, a-z, a-zo, arr, arü, azk, altz, beh, b-l, bida, g-zo, soh, sarr, ünd, esp, etxa, gab, g-i, get, hau, iba, ida, ilh, izt, l-b, laka, lan, larr, las, l-z, mak, mar, mau, leko, men, mend, mug, müs, izu, pag, arro, s-g, d-g, d-i, suh, ath, u-g, ure, urr, uzt, bil), utsi (g-z, ito, lig, l-s, o-z, irur, z-p), utzi (ahe, alo, ame, arb, beho, ürr, azka, ban, bar, bas, bid, bir, bes, d-s, d-b, esk, ezt, etx, gar, hal, haz, hoz, iho, izp, jat, lak, lekb, larz, l-a, l-o, luk, meh, mit, d-l, sar, zur, dono, bai, u-h, urk, mil), uzi (ur-p), uzteco (uzt), uzten (iri, dom)

uzta (9): Ustac (lar), usten (alo, irur), uzten (ürr, hal, jat, osp, urd, arro)

uztail (145): Üztallako (d-b), Ousstaillaco (bit), oustailléco (hen), Oustailleco (hel), Oustaileco (luh), Oustaillaco (ost), Oustailleco (bun), Oustarilaco (arh), Oustarilako (lekb), Ousthailla (altz), Ouztailé (lah), Ouztaïlako (don), ouztaïlan (zar), Ouztaïlaren (sen), Ouztaileco (arm, buz, dona), Ouztaileco (izt), Ouztaillaco (jut), Ouztaril (ain), Ouztarilako (a-s), Ustailla (hau, ora), Ustailaco (alt, kan, g-z, etx, lig, urd, o-z), Ustaillaen (ath), Ustaileko (arb, arr), Ustaillaco (a-zo, l-s), ustaicaco (ame), Ustail (esp), ustaila (hoz), Ustailaco (azk), Ustailako (l-o, mend), Ustaileco (ban, jat, lar, u-g), Ustailhako (l-b), Ustailla (amo, bard, izu), Ustaillaco (gab, dono), Ustaillako (ilh), Ustailleco (luk), Ustaril (mak), Ustarila (ainh, mau), ustarilaco (sarr), Ustarilaco (arü, b-l, etxa, larr, l-a, mar, pag, d-i, u-h, z-p), Uztaïlaco (be-l, g-zo, ida, laka, l-z, müs, bai, irur), Uztaillako (alo, gar, garr, jats, bil), Uztaillaren (haz), Uztaileco (meh), uztaileko (aiz), Uztaileko (ahe), Uztaillako (a-z), Uztail (mug), Uztaila (uzt), Uztailaco (anh, ürr, d-s, soh, ezt, iho, ort, d-l, dom), Uztailako (bar, mit, arro, d-g, urk), Uztailaren (lek, men), Uztaileco (aih, bas, bid, bes, hal, iri, its, leko, suh), Uztaileko (arn, hir, sar, zur), Uztailiako (zib), Uztailla (gam, larz), Uztaillaco (izp, lan), Uztaillaco-atjalde (iba), Uztaillako (am, beh, beho), Uztailleco (bir, iru, ur-p, urr), Uztailleko (azka, bida, get), uztarilaco (ünd), Uztarilaco (esk, lak, las, osp, s-g, zal, ure), Uztarilaren (g-i), Oustaïl (aha), Oustaïleacoarraxaldi (eih), ouztaillan (aint)

xorta (1): chortabat (lar)

xume (5): chume (zib, urr, urr, urr), chumetto (d-l) 
zabal (5): çabal (bida), çabalbat (esp, urd), zabal (hoz, sar)

zabaldegi (1): chabaldégui (hen)

zabaldu (61): çaalcea (a-zo, ilh), çaaltcéa (a-z), çaaltcea (izu), çaaltcera (g-zo), çabalcéa (ainh), çabalcea (alt, men, urd), çabalcera (g-i, larr, mau, pag), çabalcerat (ald), çabaltéra (don), çabaltcéa (hau), çabaltcea (arü, osp), çabaltcera (amo, b-l, be-l, soh, etxa, ida, laka, lekb, müs, ora, arro, s-g, zal, ure, bil), çabaltcerat (d-i), çabaltera (ath), çabaltu (urd), çabaltzera (anh, l-a), çalcea (l-b), zaaltzea (larz), zabalcea (ünd), zabalcera (arh, lan), zabaldu (aiz, aiz), zabaltcea (sarr), zabaltcera (suh), zabaltcerat (lak), zabaltzéra (u-h), zabaltzea (buz), zabaltzen (bar), zabaltzera (ame, esk, gar, izp, mit), zabaltzeratu (alo), zabalzera (a-s), zabalzerat (luk), zalazi (garr)

zaban (1): zaban (aiz)

zahar (461): çahar (ain, aint, a-m, eih, zar, ainh, ainh, amo, amo, anh, a-z, a-zo, arm, arü, arü, azk, altz, altz, beh, b-l, be-l, bit, bid, bid, bida, lek, g-z, g-z, soh, esp, etx, etx, etxa, gab, g-i, g-i, haz, hau, hau, hen, hoz, iba, iba, ida, ilh, iru, izt, its, jut, l-b, laka, laka, lig, lig, lah, lan, larr, ald, osp, l-z, l-z, l-a, l-a, mau, leko, men, men, müs, müs, ora, ort, pag, pag, arro, s-g, s-g, d-g, d-i, sen, don, zal, dona, ath, ath, irur, irur, ure, urr, bil, bil), çahara (lig, l-a, l-a, irur), çaharac (azk, azk, etx, ida, lig, l-z, mar, müs), çaharak (a-m), çaharbat (lek, lar, l-s, mar, mug, hir), çaharra (a-z, a-z, jut, jut, lar, osp, osp, leko, leko, pag, pag, bil, bil), çaharrac (ain, ain, ainh, amo, anh, a-z, a-zo, a-zo, arh, arm, arm, arü, arü, b-l, b-l, be-l, eih, lek, g-zo, g-zo, soh, etxa, etxa, gab, gab, g-i, hel, haz, hau, hen, hoz, iba, iru, iru, izt, its, its, jut, jut, lah, lan, larr, lar, lar, las, ald, ald, osp, osp, l-a, mak, mak, mau, leko, men, mend, mug, ora, ora, ort, ort, pag, arro, s-g, d-g, d-g, d-i, sen, hir, hir, zal, zal, dona, dona, ath, irur, ure, ure, urr, urr), çaharrak (altz, beh, beh, bard, bard, bit, bida, esp, ilh, ilh, l-b, l-b, laka, don, bil), çahartiac (zar, anh, a-z, bit, hoz, lan, larr), çahartiak (don), cahar (be-l, soh), cahara (irur), caharac (mar), caharrac (alt, bir, mug), chahar (aha, azka, azka, bar, beho, buz, hel, lekb, las, luh, luh, luh, luh, luh, meh, meh, izu, izu, d-g, sar, bai, mil), chaharac (urd), chaharbat (alt, urd), chaharra (izt, izt), chaharrac (alt, azka, bas, beho, izt, las, meh, izu), chaharrak (buz, buz, esp), chararac (urd), sahar (ban, kan, get, ito, luh, mau, mend, mit, mit, ost, ost, o-z, o-z, dono), sahara (l-o, l-o, urd), saharac (kan, kan, g-z, l-s, l-s, ost, o-z), saharak (l-o), saharbat (bun, l-o, z-p), saharra (mit, mit), Saharra (hir), saharrac (aha, ban, ban, bun, hen, ito, ito, lah, mend, dono, z-p, z-p), saharrak (aiz, ürr, mit), sahartiac (bun, l-o), saharturic (lig), zahar (aiz, ahe, alo, ame, ame, arb, arn, arr, arh, ürr, ür, a-s, aih, aih, bar, bas, bir, bes, d-s, sarr, zib, zib, d-b, esk, ezt, gam, gar, gar, garr, hal, iho, iri, iri, iri, iri, izp, jat, jats, lak, lekb, larz, larz, luk, luk, d-l, zur, suh, suh, u-g, u-h, ur-p, uzt), zaharac (lak, lak, lekb, urk), zaharbat (alo, a-s, ünd, urk), zaharc (urk), zaharra (get, get, iri, luk), zaharrac (aiz, aih, arn, beho, bid, bir, bes, bes, sarr, ünd, ünd, esk, ezt, get, hel, hal, iho, iri, izp, izp, jat, jats, larz, luk, dono, bai, bai, dom, dom, suh, u-g, u-g, u-h, u-h, ur-p, ur-p, uzt, uzt), zaharrak (ahe, ahe, alo, arb, arb, arr, arr, a-s, bar, d-s, d-s, sarr, zib, d-b, d-b, ezt, gam, gam, gar, garr, jats, leko, d-l, sar, sar, zur, zur, mil, mil), zaharric (esk), zahartiac (iho), zarrac (iri)

zaharkitu (3): zaharkituac (hal, jat), zaharkituak (zib)

zahartu (10): çahartiac (a-m, aint), zahartiac (arn), çahartouac (sen), çahartuac (d-i), çahartuak (bida), çaharturic (ida, arro), zahartuac (get, d-l)

zain egon (2): çaïndago (mug), zaindô (zur) 
zaindu (57): Çaïntcén (ain), Çaintcen (aint, zar, sen, ure), çaïntux (lar), çaïntzentu (mug), çaincen (leko), Çaincen (mak), çaintcen (hir), Çaintcen (amo, anh, azk, eih, its, ora, urr), Çaintcentu (esp), Caintcen (bida), Ceincentu (ort), Saïntcen (hen), saïntsen (kan), Saïntsen (kan), Saïntzen (lah), Saintcen (d-i), Saintzen'tu (ban), Saintzentzi (hoz), Seintsen (luh), Tzeintzen (hel), zaïntcen (arr), zaïntzen (u-h), zaîntzen (bai), Zaïntzen (arb, gam, haz, lekb), zaincen (ur-p), Zaincen (bir), Zaintcen (get, dono), zaintzen (lek, dom), Zaintzen (ahe, arh, aih, bid, ezt, lak, lan, sar, urk), zaintzentitzu (bes), Zaintzentizu (bes), Zaintzentu (azka, zur), Zaintzentzi (iho)

zaintzaile (65): çaïn (ure), çaïntçailez (sen), çaïntçaléic (aint, zar), çaïntçaleric (mug, ure), çaïntcaleric (amo), çain (azk, esp), çainçailleric (urr), çainçale (azk), çaintçailleric (sen), çaintçaille (bida), çaintçaléric (ain), çaintçaleric (eih, its, ora), çaintceleik (esp), çaintzaleric (iru), caïntzaléric (lah), saïntsaléric (kan), sainçaleric (mak), sainsailéric (hen), sainsale'ic (ban), tzeintzaleic (hel), zaïn zale (u-h), zaïntzaille (arb), zaïntzaile (arr), zaintzale (haz, bai), zaintzalerik (lekb), zaiñtzalerik (zib), zain zale (arh), zain zales (get), zain (hoz, lan, dom), zainçaleik (leko), zaintçaleic (lan), zaintzaileric (meh), zaintzailerik (sar), zaintzale (ahe, lek), zaintzaleic (bid, bes), zaintzaleik (iho, larz, zur), zaintzaleric (ezt, hoz, lak), zaintzalerik (d-l), zaintzalez (iho), zaintzaliez (zur), zainzaile (uzt), zainzailec (uzt), zainzalec (iri), zainzaleic (bir), zainzaleric (aih), zainzalerik (d-s), zainzalez (sar), zeintzaileik (bas), zeintzale (dono), zeinzaleric (arn)

zakur (122): çacur (amo), çakhour (ain, eih), çakhur (mug, hir), çakhurrac (lar), çakour (ort), çakourraz (don), çakur (anh, bida, lek, haz), cakhur (altz), chacour (lekb), chacourrez (arm, ald), chacur (azk, ban, bid, bun, ito, lak, men, d-i, u-g, urr), chacurac (mar, o-z), chakhour (aha, a-m, zar, kan, iba, jut, ora, ost, dona), chakhourrez (hel), chakhur (aint, aiz, ame, arn, a-z, arb, a-zo, arr, azka, beho, bes, buz, g-z, g-zo, ezt, etxa, gab, hoz, ilh, iri, iru, izt, l-b, larz, luk, leko, izu, pag, d-g, bai, suh, mil, bil, z-p), chakhurrak (laka), chakhurrés (l-s), chakhurrez (arh, beh, garr, lan, las, u-h), chakour (a-s, bit, gam, sen), chakuac (get), chakur (arü, b-l, d-s, esp, izp, jats, meh, mend, urd, sar, dono), sakhour (hen), sakhourrez (lah), sakhur (bard, bas, its, l-o, mak, dom), sakourek (luh), sakur (bir, ur-p), tçakur (ure), zacurrez (aih), zakhür (d-b), zakhur (ahe, sarr, zib, ünd, hal, iho, jat, d-l, urk, uzt), zakur (zur)

zalhe (1): salhé (o-z)

zalu (6): çalhe (laka, l-a), çalhu (las), salhu (etxa, l-o), zaluki (uzt)

zanga (2): jangaz (lar), saïngaz (ahe)

zangatu (1): sangatuac (ur-p)

zartatu (2): zartarazterainokoa (ahe), zartasteco (izu)

zein (178): -çoina (arh), çein (ur-p), çoïn (hau), çoïna (bit, bit, bun, bun, hen, iru, lah), çoïnac (a-m, eih, bit, bit, bun, hen, ora, ort, sen), çoin (a-m, arü, bid, hau, ora, pag, pag, d-g, u-g, z-p), çoina (a-m, azk, b-l, lek, esp, hoz, hoz, its, jut, jut, ort, ort, ort, dona), çoinac (azk, eih, lek, bun, hoz, hoz, izt, jut, jut, jut, jut, mak, mar, mend, mend, ort, sen, dona, dona, dona, u-h), çouïn (hau), çougn (altz, bard, g-z, g-z, g-z, hau, hau, lig, lig, lig, irur), çougnac (mau), çougnak (men), çougnec (amo, hau, hau, l-a, müs, pag), çougnek (men), çouiñ (mau, mau), çouign (a-zo, g-zo), çouignec (etxa), çouin (amo, be-l, be-l, soh, etxa, l-a, l-a, l-a, mau, müs, müs, müs, s-g, s-g), çouina (o-z), çouinbat (alt), céin (a-z), cein (soh, l-b), ceina (urr), ceinac (ame), ceinec (urr), ceinez (izt), coïnac (bun), coimbat (mar), coin (mar), coinac (izt), couiñ (arro, arro), couin (men, 
men), soin (luh, luh), (soin (luh), soina (ban), souina (ost, ost), souïnac (ost), souïnaq (ost), sougn (irur, irur), sougna (mit), sougnek (gar), souin (ürr), soumbat (ito, l-s, irur), soungn (l-s), sungn (ath), zein (ürr), zeina (sar), (zeneta (garr), zoïn (arh, ünd), zoïna (aiz, lek, haz, haz, jats), zoïnac (arh), zoïnak (haz, jats, jats), zoïnec (u-h), zoiñ (sarr), zoin (hal, jat, u-h), zoina (ame, azka, bas, bir, d-s, zib, hal, jat, lan, dono, dono), zoinac (dono, dono, ur-p), zoinec (arh), zouïnac (ost), zouin (esk)

zein ere (1): zouynere (a-s)

zelai (21): célhai (ain), celai (esp), celaietaco (bida), celaya (iri), celhai (anh, iru, d-g), celhai (get, u-g), celhay (bas, ald, eih, ure), selhai (ban), zelaïc (bas), zelai (zib), zelaik (aiz), zelarik (aiz), zelhaï (aih), Zelhai (ahe), zelhai (d-l)

zenbait (264): çémbéit (hen), çembeit (ort), çenbeit (ort), çoïnbat (bit), çoïnbeit (hel), çoimbatetan (larr), çoimbeit (its), çombaiit (a-m, amo, bit, hen, iba, ora), çombéit (a-z), çombaitétan (don), çombéitétan (a-z), çombaitetan (arü), çombéitetan (iba), çombait (aint, anh, eih, hoz, hoz, larr, larr, mar, mug, izu, dona, suh, u-g, mil, z-p), çombaitétan (z-p), çombaitetan (leko, mend), çombat (beh, bit, its, larr, ald, ora, u-g, z-p), çombeit (arm), çombeit (b-l, esp, ilh, izt, izt), çombeitetan (its), çonbaït (don), çonbaïtez (don), çonbaït (don), çonbaitetan (izu), çoubeit (bard), çouimbaitetan (be-l), çoumbaït (g$z$, etxa, hau, hau, lig, l-z, l-z, men, müs, o-z, pag, pag, pag, ath, bil), çoumbaïtetan (altz, soh, l-z, men, s-g, s-g, bil), çoumbait (ainh, be-l, g-zo, soh, g-i, laka, lig, osp, l-a, l-a, mau, zal, zal), çoumbaitetan (ainh, g-i, ida, ida, laka, osp, l-a, mau, o-z, arro), çoumbaytetan (etxa, etxa), çoumbeit (a-zo, bard), çounbaittetan (hau), çounbaitetan (amo), çounbeït (beh), çumbait (d-g), çumbaitetan (zal), çumbeït (gab), çumbeïtetan (gab), çumbeit (beh), cémbait (ain), cémbatï (ain), cembaït (aha, zar, lekb, lekb, dona, ure), cembaitekin (lek, lar), cembait etan (a-m), cembaitetan (aha, iru), cembait (arr, arh, lek, iru, jut, las, mak, mend, d-i, d-i, u-h), cembaitetan (las, mak), cembeit (azk, sen), cembeit (arb, azk, bida, its, sen, urr, urr, urr), cembeitetan (arb, arr), coïmbaït (mug), combait (leko), combat (mar, izu), combeit (l-b, lah), coumbaï (etx, etx, s-g, ath), coumbaitetan (etx, müs), coumbaitetan (arro), sembaitetan (l-o), senbait (l-o), sombait (ban, ban), sombeitek (luh), sonbait (kan), sonbat (kan), soumbait (irur), soumbéit (ito, ost), soumbait (mit), soumbéit (l-s), soumbaitetan (mit), sounbaitetan (ürr), sounbait (ürr), sumbatetan (mit), zünbaitetan (d-b), zünbeit (d-b), zembait (bas, lek, lak, bai), zembait (ame, beho, bid, ezt, hal, iri, jat, d-l, sar), zembaitetan (lak), zembeit (lan), zenbait (bas, beho), zoïnbatian (aiz), zoïnbeïtetan (hel), zombait (buz, buz, luk, bai), zombaiti (aiz), zombait (arn, azka, d-s, ezt, gam, iho, izp, izp, izp, dom, suh, u-g, uzt), zombaitetan (ahe, ame, azka, bid, dom), zombat (ame, d-s, ezt, izp, suh), zombeït (haz, meh), zombeit (aih, bir, zib, get, meh, zur, zur, urk, ur-p), zombeitetan (zib), zonbaït (haz, jats), zonbait (beho, bes, bes), zonbeytetan (a-s), zoumaïtetan (gar), zoumbaït (aiz, alo, alo), zoumbaitetan (sarr, ünd), zoumbait (bar, esk), zoumbaitetan (ürr, bar, esk), zoumbeit (garr), zounbait (gar), zounbeyt (a-s), zumbeit (larz)

zenbat (29): çoumat (altz), çoumbat (ida), çounbat (d-g), çumbat (gab, d-g), cémbat (ain, hen), cembat (b-l, g-zo, iru, jut, lekb, lah, lar, las, mak, ure), sénian (ito), sembat (aiz, ban), zembat (bas, bid, lak, d-l, sar), zombat (arn), zounbat (ürr, gar), zunbat (d-b)

zenbat-nahi (35): çonbat_nahi (don), çoumbat_nahi (ainh, a-zo, arü, bard, be-l, g-z, soh, etxa, g-i, laka, lig, osp, l-z, l-a, mau, men, müs, o-z, pag, arro, s-g, zal, ath, bil), zembatnahi (bai), zombatnahi (buz), Zoumbanahi (a-s), zoumbat_nahi (alo, bar, sarr, ünd, esk, 
garr, mit)

zendako (1): cendaco (aha)

zer (133): ce (aha), çer (ald), cér (ain, hen, ito, ost, don), cer (a-m, aint, arn, eih, zar, ainh, amo, anh, a-z, arb, a-zo, arr, arh, arm, arü, azk, altz, beh, b-l, be-l, bida, g-z, g-zo, soh, sarr, ünd, esp, etx, etxa, gab, g-i, get, hau, hoz, ida, iri, iru, its, jut, laka, lah, larr, lar, las, osp, l-z, l-a, luk, mak, mar, mau, meh, men, mug, müs, ora, urd, o-z, ort, izu, pag, arro, s-g, d-g, d-i, sen, hir, zal, dono, dona, suh, ath, u-h, ure, urr, uzt, bil, z-p), ser (ürr, ban, bun, iba, lig, l-s, l-o, luh, mit, irur), zer (aiz, aiz, alo, ame, a-s, azka, aih, bar, bas, beho, bir, lek, d-s, buz, zib, esk, ezt, gar, garr, hel, hal, haz, iho, izp, jat, jats, lak, lekb, lan, d-l, sar, bai, dom, u-g, urk, ur-p, mil)

zerbitzatu (86): çerbitzatu (luk), cerbiçatu (ort), cerbitçatou (hen, sen), cerbitçatu (anh, las, mak, leko, mug, ora, suh, ure), cerbitcatu (its), cerbitchatou (eih, iba, jut, ost), cerbitchatu (be-l, soh, d-i, dona), cerbitehatu (beh), cerbitzatu (bid, get, iru, meh), cerbitzatzen (iri), cerbitzatzou (lah), cerbizatu (bir), cerbuchatu (ath), cerbutchatu (ainh, a-m, amo, g-z, etxa, hau, ida, laka, larr, mau, müs, pag, s-g, bil, z-p), chérbitchatcén (don), cherbitchatou (bun, gam, d-g), cherbitchatu (a-z, beho, buz, gab, men, izu), cherbitzatu (azka), cherbuchatcen (a-zo), cherbutchatu (g-zo, ilh, l-b, mar), serbitçatu (izt), serbitchatu (mend), serbitsatu (bard, u-g), serbutchatu (lig, mit, urd, irur), zerbitchatu (jats), zerbitzatou (lekb), zerbitzatu (bas, haz, lak, zur, dom, urk, mil), zerboutchatou (a-s), zerbutchatu (alo, ürr, bar, sarr, ünd, esk)

zeren (89): çeren (luk), cérén (ain, ost, don), céren (bun, lah, lah, z-p, z-p), ceren (ainh, alt, ame, amo, arm, arü, arü, ürr, azk, azka, b-l, be-l, bir, g-zo, soh, sarr, sarr, ünd, ünd, etxa, etxa, g-i, g-i, g-i, get, get, haz, haz, izt, las, las, ald, osp, osp, l-a, l-a, l-o, mak, mau, leko, mend, müs, müs, müs, ort, pag, d-g, d-i, sen, zal, zal, dona, suh, urr, uzt), ceren (ame, osp), sérén (bun, ito), séren (irur), seren (ürr, lig, lig, luh, mit, irur, irur), zeren (bas, d-s, ezt, ezt, gar, iho, jats, lekb, lekb, lan, larz, d-l)

zerendako (4): çèndaco (bit), ceentaco (ida), cendaco (arh), zendaco (u-h)

zeren-eta (31): cêneta (1-b), ceren_eta (aiz, ahe, anh, bid, lek, gab, hoz, iru, its, jut, osp, l-a, men, mug, ora, arro, s-g, zal, dono, dom, ure, bil, lig), cereneta (urd), zeren_eta (a-s, aih, buz, lak, urk, mil)

zerratu (4): cerratcian (arn), cerratcean (b-l, izt), cerratcian (dona)

zertarako (1): certaco (mar)

zeru (1): zerurat (garr)

zezengai (1): cecenkaïez (ath)

zigorrada (1): (cigorradak (bida)

zilo (1): (silobat (z-p)

zinez (6): cinez (bil), sines (o-z), sinhes (bir, lan, ur-p), Zinez (ahe)

zipu (1): ciphu (leko)

zola (5): çola (don), chola (mil), sola (dono), zola (lan), zolan (bas)

zoratu (1): çoratubatçuez (mug)

zorigaitz (1): zorigaitzezkoak (zib)

zorion (1): zorion (zib)

zoriondun (1): zoriondun (d-1)

zorioneko (2): zorionecoac (hal, jat)

zu (2): çu (eih) Zuk (bar) 
zuek (161): Ciec (izu), Çièk (bit), Çouéc (ain), çouec (lah), Çouec (ald, sen), Çuëc (hen), çuec (hir, urr), Çuec (azk, b-l, its, lar, ort), Çuek (bida, mak), Çuen (bida), çuien (esp), çuik (esp), Cïek (don), Ciéc (iba), ciék (don), Cic (arm), ciec (a-zo, beho, etxa, hoz, ida, mar), Ciec (a-m, aha, aint, arn, eih, zar, ainh, amo, anh, a-z, arü, altz, be-l, g-z, g-zo, soh, etx, gab, g-i, hau, izt, jut, laka, larz, las, osp, l-z, mau, müs, ora, o-z, pag, arro, s-g, d-g, d-i, zal, dona, suh, ath, u-h, ure, bil, z-p), ciek (beh), Ciek (arh, sarr, ünd, ilh, l-b, leko, mend, mug, ost), Cieq (larr), cin (arh, arm, arm, arm), Cirec (urd), Cuec (iru), cuen (bida), Siéc (bun, ito, l-s), Siec (ban, lig, irur), Siek (bard, l-o, men), Souec (kan), souek (luh), Zîc (bai), ziec (luk), Ziec (ürr, bes, esk, ezt, iho, izp, jats, lak, lan, l-a, mit, dom), ziek (d-s, garr, urk), Ziek (aiz, alo, a-s, azka, buz, d-b, gam, gar, u-g), zi-ic (aih), Ziic (bid, hel), ziin (bid, hel), zi-in (aih), zin (aih), Zirek (lekb), Zuïc (bas), zuïn (bas), Zuec (ame, bir, lek, get, hal, iri, jat, meh, dono, ur-p, uzt), Zuek (ahe, arb, zib, haz, d-l, sar, mil), Zuik (arr, zur), zuin (arr, zur)

zuri (1): chouri (alo)

zutitu (60): -choutitou (ora), -chutitu (d-s), choutitcen (don), choutitou (ain, arh, bit, eih, kan, lah, ost, sen), choutitouçouçoun (arm), choutitouzen (a-s), choutitsen (ald, luh), chutitcen (esp, get, hir), chutitu (ahe, amo, a-z, azk, ban, lek, zib, gab, garr, hal, haz, hoz, iho, iri, izt, ito, jat, jats, lak, larz, luk, mak, mau, meh, mend, mug, urd, izu, arro, s-g, d-l, dono, bai, dom, u-h, ure, uzt, mil, bil), chutizen (lan), tchutitu (alo)

zuzen (4): zuzen (arb, arr, hal, jat)

zuzentasun (4): çoucéntassouna (ain), cucentassunari (lar), zuzentasunak (zib, d-l)

zuzulu (1): çuçulian (urd) 
